\title{
Perovskite oxide heteroepitaxy
}

strain and interface engineering 


\section{Cover}

The background displays a scanning transmission electron microscopy image of a $\mathrm{SrTiO}_{3} / \mathrm{La}_{0.67} \mathrm{Sr}_{0.33} \mathrm{MnO}_{3} / \mathrm{SrTiO}_{3}$ heterostructure.

\section{Ph.D. committee}

Chairman and secretary

Prof. dr. G. van der Steenhoven (University of Twente)

Supervisors

Prof. dr. ing. D.H.A. Blank (University of Twente)

Prof. dr. ing. A.J.H.M. Rijnders (University of Twente)

Assistant supervisor

Dr. ir. G. Koster (University of Twente)

Members

Prof. dr. J. Aarts (University of Leiden)

Prof. dr. M.G. Blamire (University of Cambridge)

Dr. ir. A. Brinkman (University of Twente)

Prof. dr. R. Claessen (University of Würzburg)

Prof. dr. P.J. Kelly (University of Twente)

Prof. dr. G. van Tendeloo (University of Antwerp)

The research described in this thesis was performed within the Inorganic Materials Science group, the NanoElectronic Materials group and the MESA+ Institute for Nanotechnology at the University of Twente, the Netherlands and within the Hwang Laboratory at the University of Tokyo, Japan. This research was supported by NWO, the Dutch science foundation.

\section{Hans Boschker}

Perovskite oxide heteroepitaxy, strain and interface engineering Ph.D. thesis University of Twente, Enschede, the Netherlands.

ISBN: 978-90-365-3127-6

DOI: $10.3990 / 1.9789036531276$

Printed by Wöhrmann Print Service, Zutphen, the Netherlands

(C) Hans Boschker, 2011 


\section{PEROVSKITE OXIDE HETEROEPITAXY}

\section{STRAIN AND INTERFACE ENGINEERING}

\section{PROEFSCHRIFT}

ter verkrijging van

de graad van doctor aan de Universiteit Twente, op gezag van de rector magnificus,

Prof. dr. H. Brinksma,

volgens besluit van het College voor Promoties

in het openbaar te verdedigen

op vrijdag 4 februari 2011 om 14:45 uur

$$
\text { door }
$$

Johannes Arnoldus Boschker

geboren op 21 maart 1981

te Woudenberg 
Dit proefschrift is goedgekeurd door de promotoren

Prof. dr. ing. D.H.A. Blank

Prof. dr. ing. A.J.H.M. Rijnders

en de assistent-promotor

Dr. ir. G. Koster 


\section{Contents}

1 Perovskite oxide heteroepitaxy 1

1.1 Introduction . . . . . . . . . . . . . . . . . 1

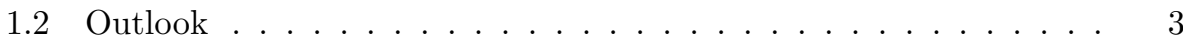

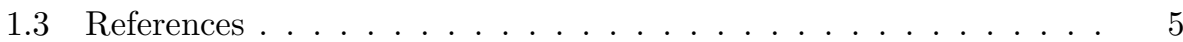

2 Conductivity at the $\mathrm{LaAlO}_{3} / \mathrm{SrTiO}_{3}$ interface 7

2.1 Introduction . . . . . . . . . . . . . . . . 7

2.2 Overview relevant literature . . . . . . . . . . . . . . . 10

2.2 .1 Theory . . . . . . . . . . . . . . . . . . 10

2.2 .2 Experiment . . . . . . . . . . . . . . . . . 11

2.3 Absence of potential buildup in the LAO . . . . . . . . . . . 12

2.3 .1 Experimental . . . . . . . . . . . . . . . . . . 12

2.3.2 Measuring the potential buildup with XPS . . . . . . . 13

2.3 .3 Summary . . . . . . . . . . . . . . . . 15

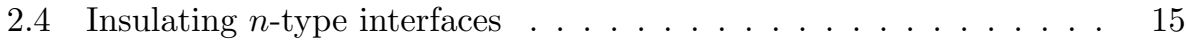

2.4.1 LAO/STO interfaces on $\mathrm{NdGaO}_{3}$ substrates . . . . . . . . 16

2.4.2 LAO/STO interfaces grown in high oxygen pressure . . . 17

2.4 .3 Conclusions . . . . . . . . . . . . . . . 20

2.5 Structural reconstruction of the polar discontinuity . . . . . . . . 20

2.6 Thickness dependence of the conductivity and induced polarization in the $\mathrm{STO} \ldots \ldots \ldots \ldots \ldots . \ldots \ldots 21$

2.6.1 Induced polarization . . . . . . . . . . . . . . . . 22

2.6.2 Thermally activated conductivity . . . . . . . . . . . 26

2.6.3 The complete picture: polarization induced conductivity . . 27

2.7 Conclusions and outlook . . . . . . . . . . . . . . . . 28

2.8 References . . . . . . . . . . . . . . . . . . . . 30

3 Fabrication and properties of $\mathrm{La}_{0.67} \mathrm{Sr}_{0.33} \mathrm{MnO}_{3}$ thin films 37

3.1 Introduction . . . . . . . . . . . . . . . . 37

$3.2 \quad \mathrm{La}_{1-\mathrm{x}} \mathrm{Sr}_{\mathrm{x}} \mathrm{MnO}_{3} \ldots \ldots \ldots \ldots \ldots \ldots \ldots \ldots$

3.2.1 Magnetic interactions between manganese ions . . . . . . . 39

3.2 .2 The phase diagram . . . . . . . . . . . . . . . 40

3.3 Thin film growth . . . . . . . . . . . . . . . . . 42

3.3.1 Substrates . . . . . . . . . . . . . . . . . 42

3.3 .2 Pulsed laser deposition . . . . . . . . . . . . . . . . . 43

3.3 .3 Growth mode . . . . . . . . . . . . . . . . . . . 43 
3.3.4 Surface morphology . . . . . . . . . . . . . . . . . 45

3.4 Crystal structure . . . . . . . . . . . . . . . . . 46

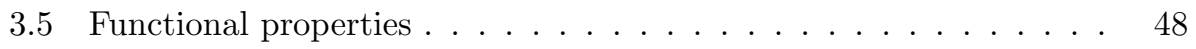

3.5.1 Magnetization . . . . . . . . . . . . . . . . . 49

3.5.2 Electrical transport . . . . . . . . . . . . . . 50

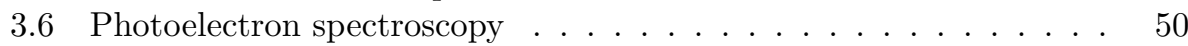

3.7 Scanning transmission electron microscopy . . . . . . . . . . . 52

3.8 Comparison to other groups . . . . . . . . . . . . . . 54

3.9 Conclusions . . . . . . . . . . . . . . . . . . . 54

3.10 References . . . . . . . . . . . . . . . 57

4 Magnetocrystalline anisotropy in strained $\mathrm{La}_{0.67} \mathrm{Sr}_{0.33} \mathrm{MnO}_{3}$ thin films 63

4.1 Introduction . . . . . . . . . . . . . . . . 63

4.2 Néel framework . . . . . . . . . . . . . . . . . . 65

4.2 .1 Model . . . . . . . . . . . . . . . . . . . . 66

4.2.2 Distortions of the cubic lattice . . . . . . . . . . . . 66

4.2 .3 Summary model . . . . . . . . . . . . . . . . . . . 69

4.3 Experiments . . . . . . . . . . . . . . . . . . . . 69

4.3 .1 Crystal structure . . . . . . . . . . . . . . . . . . . . 69

4.3 .2 Experimental results . . . . . . . . . . . . . . 70

4.3.3 Analysis of the experiments in terms of the Néel model . . 73

4.3.4 Summary experiments . . . . . . . . . . . . . . . 77

4.4 Microscopic origin of the anisotropy $\ldots \ldots \ldots \ldots \ldots 77$

4.4 .1 Single ion model . . . . . . . . . . . . . . . 78

4.4.2 Oxygen octahedra rotation and deformation . . . . . . 8 80

4.4 .3 Implications for LSMO . . . . . . . . . . . . . . . . . 83

4.5 Conclusions . . . . . . . . . . . . . . . . . . . 83

4.6 References . . . . . . . . . . . . . . . . . 85

5 Interface engineering at the $\mathrm{La}_{0.67} \mathrm{Sr}_{0.33} \mathrm{MnO}_{3} / \mathrm{SrTiO}_{3}$ interface 89

5.1 Introduction . . . . . . . . . . . . . . . . . 90

5.2 Experimental method for the study of interface properties . . . . 92

5.3 Polar discontinuities . . . . . . . . . . . . . . . . . . . 93

5.3.1 LSMO/STO interface configurations . . . . . . . . . . . 94

5.3 .2 Experimental . . . . . . . . . . . . . . . . . 99 96

5.3 .3 Results . . . . . . . . . . . . . . . . . . . . . . . 98

5.3 .4 Discussion . . . . . . . . . . . . . . . . . . . . . 102

5.3 .5 Conclusions . . . . . . . . . . . . . . . . . . 102

$5.4(110)_{\mathrm{pc}}$ oriented interfaces $\ldots \ldots \ldots \ldots \ldots \ldots \ldots$

5.4 .1 Crystal structure . . . . . . . . . . . . . . . . . . 103

5.4 .2 Functional properties . . . . . . . . . . . . . . 105

5.4 .3 Discussion . . . . . . . . . . . . . . . . . . . . . 106

5.4 .4 Conclusions . . . . . . . . . . . . . . . . . . . 108

5.5 Order and disorder at the interface . . . . . . . . . . . . . 109

$5.5 .1 \quad$ Experimental . . . . . . . . . . . . . . . . . 110

5.5 .2 Results . . . . . . . . . . . . . . . . . . . . 111 
5.5 .3 Conclusions . . . . . . . . . . . . . . . . . . . 113

5.6 Mechanism of the reduction of the properties at LSMO interfaces . 114 5.6 .1 Overview of the results . . . . . . . . . . . . 114

5.6 .2 Discussion . . . . . . . . . . . . . . . . . . . 115

5.6 .3 Conclusions . . . . . . . . . . . . . . . . . . 117

5.7 References . . . . . . . . . . . . . . . . 118

6 Epilogue 125

6.1 References . . . . . . . . . . . . . . . . . 127

A Determination of the Curie temperature 129

A.1 Magnetization measurements . . . . . . . . . . . . . . . . 129

A.2 Transport measurements . . . . . . . . . . . . . . . . 131

A.3 Conclusion ....................... . . 133

A.4 References . . . . . . . . . . . . . . . . . 134

$\begin{array}{ll}\text { Summary } & 135\end{array}$

$\begin{array}{ll}\text { Samenvatting } & 139\end{array}$

$\begin{array}{ll}\text { Dankwoord } & 141\end{array}$ 



\title{
Chapter 1
}

\section{Perovskite oxide heteroepitaxy}

\begin{abstract}
This chapter gives a brief introduction to perovskite oxide heteroepitaxy. Strain and interface engineering are identified as the fundamental tools in heteroepitaxy for the controlled study and manipulation of materials properties. Several examples of heteroepitaxy in general and perovskite oxide heteroepitaxy in particular are discussed. Furthermore, an outline of the structure of the thesis is presented.
\end{abstract}

\section{$1.1 \quad$ Introduction}

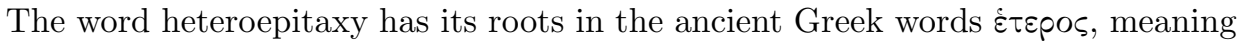
"other" or "different", $\varepsilon \pi \iota$, meaning "above" or "upon", and $\tau \dot{\alpha} \xi \iota \varsigma$, meaning "in ordered manner" or "arrangement". So epitaxy can be translated as "to arrange upon" [1]. In materials science, it is used to indicate the deposition of thin films which are structurally ordered with the underlying substrate. Heteroepitaxy is then the epitaxy of films of a different material than the substrate.

For certain classes of materials, the heteroepitaxy can nowadays be controlled to single atomic layers. In this case heteroepitaxy is no longer just the stacking of different layers, but it has become a materials scientist's tool to study existing materials in the atomic limit. Artificial materials can be created as well; when the thicknesses of the consecutive layers in a heterostructure approach the atomic limit, the heterostructure can have properties not present in the building block materials. 
The new functionality of the heterostructures can be attributed to two fundamental effects in heteroepitaxy. At first the crystal structure of the layers is changed, due to the matching of the in-plane lattice constants to those of the substrate. The matching results in strain in the layers, the magnitude of which can be controlled with the use of an appropriate substrate. This is called strain engineering. Furthermore, the interfaces between different layers break symmetry and therefore new functionality can be expected at the interfaces. Heteroepitaxy allows for direct intervention, e.g. with dopant insertion, at the interface during growth, which is called interface engineering.

An important example is the fabrication of high mobility two-dimensional electron gases in GaAs/AlGaAs heterostructures. In order to prevent impurity scattering, the dopant ions have to be separated from the transport channel. This is realized by growing a modulation doped heterostructure. Here the heteroepitaxy enables control over the position of the dopant layer [2], away from the transport channel which is formed at the GaAs/AlGaAs interface. The improvement in charge carrier mobility in two dimensional electron gases has led to the discovery of the fractional quantum hall effect [3].

The coupling of the magnetization between different Fe layers in Fe/Cr heterostructures is another example of the new physics enabled by heteroepitaxy. The antiferromagnetically coupled magnetization in the Fe layers can be ferromagnetically aligned with a small magnetic field and this changes the resistivity of the heterostructure, i.e. the giant magnetoresistive effect $[4,5]$. Nowadays, this effect is widely used by hard disk drive manufacturers to read magnetic memory bits.

Perovskite oxides are an extremely interesting class of materials. The perovskite $A B \mathrm{O}_{3}$ crystal structure can be realized with a wide variety of different elements at the $A$ and $B$ positions. This results in a class of materials with similar lattice parameters but a wide range in properties, from dielectrics and piezoelectrics to ferroelectrics, from semiconductors, transparant conductors and metals to superconductors and from antiferromagnetic to ferromagnetic and multiferroic materials. Perovskite oxides are therefore naturally suitable for heteroepitaxy [6]. An example of a perovskite oxide heterostructure is shown in figure 1.1. Perovskite oxide heteroepitaxy is nowadays a large field of research and significant advances have been made to realize even more functionality from the perovskite building blocks.

An interesting example can be found in the work of Lee et al. [7] who created a superlattice of $\mathrm{BaTiO}_{3}, \mathrm{SrTiO}_{3}$ and $\mathrm{CaTiO}_{3}$ in which each individual layer was only 2 unit cell layers thick. $\mathrm{BaTiO}_{3}$ is a ferroelectric and $\mathrm{SrTiO}_{3}$ and $\mathrm{CaTiO}_{3}$ are dielectrics, but the heterostructure as a whole is ferroelectric with a larger polarization than that expected from $\mathrm{BaTiO}_{3}$ embedded in a paraelectric matrix. This is explained by the breaking of the inversion symmetry by the three component superlattices as well as the strain in the heterostructure, both of which enhance the polarization.

Another example is the work of Gozar et al. who have studied interfaces between undoped and overdoped $\mathrm{La}_{2-\mathrm{x}} \mathrm{Sr}_{\mathrm{x}} \mathrm{CuO}_{4}$ (LSCO) [8]. At optimum doping, $x=0.15$, 


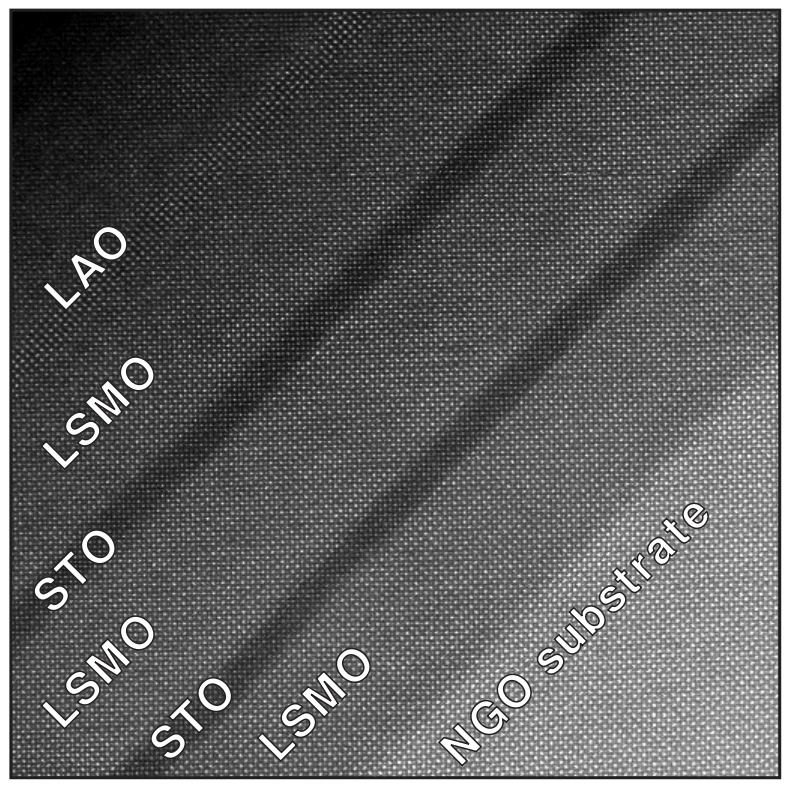

Figure 1.1: An example of a perovskite oxide multilayer structure with layers of $\mathrm{La}_{0.67} \mathrm{Sr}_{0.33} \mathrm{MnO}_{3}$ (LSMO), $\mathrm{SrTiO}_{3}$ (STO) and $\mathrm{LaAlO}_{3}$ (LAO) grown on a $\mathrm{NdGaO}_{3}$ (NGO) substrate. This image was measured at Chalmers University of Technology, Sweden.

LSCO is a superconductor with a critical temperature of $40 \mathrm{~K}$. The interface fabricated by Gozar is superconducting at $50 \mathrm{~K}$, but only in a thin region. The charge distribution at the interface is not abrupt and subsequent research showed that a $\mathrm{CuO}_{2}$ plane a few layers removed from the interface on the nominally overdoped side had exactly 0.15 doping and was responsible for the enhanced superconductivity. The research method to identify this superconducting layer deserves further attention. The researchers alloyed a specific $\mathrm{CuO}_{2}$ plane with 3 $\%$ of $\mathrm{Zn}$, which reduces superconductivity. By systematically alloying every $\mathrm{CuO}_{2}$ plane in the heterostructure individually, they found that the superconductivity only disappeared if one specific layer was alloyed [9].

These examples show the type of research which becomes possible if layers of materials can be grown atomic layer for atomic layer. In this thesis an exploration of the possibilities with strain and interface engineering is made for both the $\mathrm{LaAlO}_{3} / \mathrm{SrTiO}_{3}(\mathrm{LAO} / \mathrm{STO})$ interface and the fully spin polarized metal $\mathrm{La}_{0.67} \mathrm{Sr}_{0.33} \mathrm{MnO}_{3}$ (LSMO).

\subsection{Outlook}

Chapter 2 of this thesis is concerned with the interface between LAO and STO. This heterointerface between two band insulators has intriguing physical proper- 
ties, most especially the atomic stacking sequence dependent conductivity. This conductivity is commonly explained by an electronic reconstruction induced by the polar nature of the interface. However, the experiments presented in this thesis, the dependence of the conductivity on strain and oxygen deficiency and the absence of the expected electrostatic potential buildup, show that this understanding is debatable. An alternative model is proposed in which the conductivity is governed by the strain induced polarization in the STO.

Chapter 3 describes the growth and properties of the LSMO thin films. In order to study the intrinsic properties of the LSMO films and interfaces, high quality heteroepitaxy is crucial. The characterization presented in this chapter demonstrates that the LSMO thin films have properties similar to high quality bulk LSMO crystals and are at least as good as the samples found in literature.

In chapter 4 the dependence of the magnetization of LSMO on the applied strain is presented. It is shown that the magnetic anisotropy can be controlled with the strain applied by an appropriate substrate surface orientation. The results can be described with the use of a model originally developed by Néel. The signs of the Néel parameters are unambiguously identified by comparing the Néel model with the experimental data. The microscopic origin of the magnetocrystalline anisotropy is discussed in terms of the single ion model of anisotropy. The Néel parameters can be described in terms of $\mathrm{Mn}-\mathrm{O}$ bond length variations and oxygen octahedra bond angle variations. In order to describe the observed strain dependence of the anisotropy correctly, oxygen octahedra bond angle variations with applied strain are required.

Chapter 5 discusses the attempts to change the properties of the LSMO at the LSMO/STO interface with interface engineering. First, the effect of the polar discontinuity analogous to the LAO/STO interface was investigated. The polar discontinuity was removed with local changes in the doping of the LSMO layer. The results show an increase in functional properties in samples without polar discontinuities. Then, the effect of the crystal orientation was investigated. In the (110) orientation, LSMO has improved magnetization, but a reduced conductivity. The final interface engineering scheme tested the effect of chemical disorder at the interface. Samples without disorder at the interface were created with the use of a $\mathrm{SrMnO}_{3}$ (SMO) layer. The required electrons for the SMO to become ferromagnetic were supplied by modulation doping from La dopants in the STO away from the interface. Preliminary results indicate that the modulation doping of the interface was achieved but no improvement of the functional properties was realized.

The final chapter is an epilogue, in which the results are discussed in a wider perspective. Furthermore, an outlook towards the challenges in perovskite oxide heteroepitaxy in the near future is given. 


\subsection{References}

[1] "Epitaxy." http://en.wikipedia.org/wiki/Epitaxy (2010).

[2] R. Dingle, H. Stormer, A. Gossard, and W. Wiegmann, "Electron mobilities in modulation-doped semiconductor heterojunction super-lattices," APPLIED PHYSICS LETTERS, vol. 33, pp. 665-667, 1978.

[3] D. Tsui, H. Stormer, and A. Gossard, "Two-dimensional magnetotransport in the extreme quantum limit," PHYSICAL REVIEW LETTERS, vol. 48, pp. 1559-1562, 1982.

[4] P. Grunberg, R. Schreiber, Y. Pang, M. Brodsky, and H. Sowers, "Layered magnetic-structures - evidence for antiferromagnetic coupling of Fe layers across $\mathrm{Cr}$ interlayers," PHYSICAL REVIEW LETTERS, vol. 57, pp. 2442-2445, 1986.

[5] M. Baibich, J. Broto, A. Fert, F. Vandau, F. Petroff, P. Eitenne, G. Creuzet, A. Friederich, and J. Chazelas, "Giant magnetoresistance of $(001) \mathrm{Fe} /(001) \mathrm{Cr}$ magnetic superlattices," PHYSICAL REVIEW LETTERS, vol. 61, pp. 2472-2475, 1988 .

[6] G. Rijnders and D. H. A. Blank, "Materials science - Build your own superlattice," NATURE, vol. 433, pp. 369-370, 2005.

[7] H. Lee, H. Christen, M. Chisholm, C. Rouleau, and D. Lowndes, "Strong polarization enhancement in asymmetric three-component ferroelectric superlattices," NATURE, vol. 433, pp. 395-399, 2005.

[8] A. Gozar, G. Logvenov, L. F. Kourkoutis, A. T. Bollinger, L. A. Giannuzzi, D. A. Muller, and I. Bozovic, "High-temperature interface superconductivity between metallic and insulating copper oxides," NATURE, vol. 455, pp. 782-785, 2008.

[9] G. Logvenov, A. Gozar, and I. Bozovic, "High-Temperature Superconductivity in a Single Copper-Oxygen Plane," SCIENCE, vol. 326, pp. 699-702, 2009. 


\title{
Chapter 2
}

\section{Conductivity at the $\mathrm{LaAlO}_{3} / \mathrm{SrTiO}_{3}$ interface}

\begin{abstract}
The conducting interface between the two band insulators $\mathrm{LaAlO}_{3}(\mathrm{LAO})$ and $\mathrm{SrTiO}_{3}$ (STO) is studied in this chapter. The main experimental result is that the expected electrostatic potential buildup in the LAO layer is not observed. Furthermore, samples grown on other substrates than STO and samples grown at high oxygen pressure are insulating. The results indicate that the most widely used model to explain the conductivity at the interface, the electronic reconstruction due to the polar discontinuity, is not applicable to the experiments. An alternative model for the conductivity is proposed, in which the polar discontinuity is compensated by structural reconstructions, details of which depend on the growth conditions. At certain growth conditions, the structural reconstructions result in charge transfer to the STO and the charge in the STO resides in impurity states within the bandgap. Conductivity occurs when the induced polarization in the STO activates the charge carriers to the conduction band.
\end{abstract}

\section{$2.1 \quad$ Introduction}

In 2004, Ohtomo and Hwang published their results on polar interfaces between two perovskite band insulators, $\mathrm{LaAlO}_{3}(\mathrm{LAO})$ and $\mathrm{SrTiO}_{3}$ (STO) [1]. LAO consists of $\mathrm{LaO}$ and $\mathrm{AlO}_{2}$ layers with a net charge (in the ionic limit) of \pm 1 and STO consists of charge neutral $\mathrm{SrO}$ and $\mathrm{TiO}_{2}$ layers, when viewed along the (001) direction. This difference results in a polar discontinuity at the interface between the two materials. This interface was found to be conducting if the atomic stacking sequence at the interface is $\mathrm{SrO}-\mathrm{TiO}_{2}-\mathrm{LaO}-\mathrm{AlO}_{2}$ (n-type) and insulating if the se- 
quence is $\mathrm{TiO}_{2}-\mathrm{SrO}-\mathrm{AlO}_{2}-\mathrm{LaO}$ ( $p$-type). The dependence of the conductivity on the termination of the interface was explained with an electronic reconstruction of the polar discontinuity. In short, the polar discontinuity results in a divergence of the electrostatic potential which has to be compensated. The sign of the divergence, and therefore the possible reconstruction mechanisms, depends on the atomic stacking sequence. For the $n$-type interface, the diverging potential is removed if half an electron per unit cell is present at the interface. Similarly, the $p$-type interface does not have a diverging potential if the interface is hole-doped. The titanium ions at the $n$-type interface can easily be electron doped due to the multiple valence states of the titanium. The intrinsic electron doping of the interface by the polar discontinuity is called electronic reconstruction and it explains the observed conductivity of the $n$-type interface. At the $p$-type interface, the intrinsic hole doping is suppressed by the presence of oxygen vacancies and therefore no conductivity has been found at this interface [2].

After the discovery of the conductivity at the LAO/STO interface, research has focussed on the electronic properties. The carrier density and mobility are found to depend on the thickness of the LAO layer and the growth conditions [3-9]. The interface can be superconducting $[10]$ or show magnetic scattering $[8,11]$ at low temperature. The low temperature transport properties can be controlled with electric field effect doping $[12,13]$, which can be used to realize devices $[14,15]$.

The mechanism of electronic reconstruction by a polar discontinuity is quite general and can be expected to occur at a wide variety of transition metal compound interfaces. Even so, most research has focussed on the LAO/STO interfaces and other examples are rare. Experiments have been performed at the interface between $\mathrm{LaMnO}_{3}$ (LMO) and $\mathrm{SrMnO}_{3}$ (SMO) [16-18], $\mathrm{La}_{2} \mathrm{CuO}_{4}$ (LCO) and $\mathrm{La}_{1.55} \mathrm{Sr}_{0.45} \mathrm{CuO}_{4}$ (LSCO) [19], $\mathrm{LaGaO}_{3}$ and STO [20] and $\mathrm{LaVO}_{3}$ (LVO) and STO [21]. For the manganite and cuprate interfaces, the doped charge is distributed in a relatively broad region of the interface and the properties depend on the local doping concentration. The LGO/STO and LVO/STO interfaces have the same transport properties as the LAO/STO interface. The interfaces between $\mathrm{KTaO}_{3}$ (KTO) and STO [22] and amorphous $\mathrm{CaHfO}_{3}$ (CHO) and STO [23] have also been reported to be conducting. KTO is a polar material with negative net charge at the $A \mathrm{O}$ layer and therefore the electronic reconstruction mechanism should result in $p$-type conductivity at the interface. $N$-type conductivity was found. The amorphous $\mathrm{CHO}$ is nonpolar material and conductivity cannot be explained with the polar discontinuity model. It is either due to strain or to oxygen vacancy doping. Also for LAO/STO interfaces, it was shown that, at least for samples grown at low oxygen pressure, the dominant doping is due to oxygen vacancies and not due to electronic reconstruction $[5,7,8]$.

Four models have been presented to describe the conductivity of the LAO/STO interface. The first model is based on the electronic reconstruction scenario and it is an extension of the explanation given by Ohtomo [1]. The physics can be understood in terms of a simple electrostatic model $[24,25]$. The polar discontinuity at the interface results in an electrostatic potential buildup in the LAO layer. At a critical thickness the energy of electrons in the valence band at the LAO surface is 
higher than the available states in the STO conduction band and charge transfer occurs. The second model is based on oxygen vacancies [5, 7]. During the growth of the LAO film, high energy La ions sputter oxygen ions from the substrate and this results in conductivity in the top layer of the STO. One of the main drawbacks of the oxygen vacancy model is that the termination dependence of the conductivity is unexplained. The third model is based on intermixing of the La and $\mathrm{Sr}$ ions in the top layer of the STO [26]. La ions are then present in the STO and result in electron doping of the STO. In this model, the termination dependence is not explained either. Finally, a model based on a polarization discontinuity was proposed [27]. At the interface a discontinuity exists in the polarization of the materials which results in narrowing of the bandgap. Therefore, defect induced charge carriers accumulate at the interface, resulting in local conductivity. This is similar to the model for the conductivity at domain walls in $\mathrm{BiFeO}_{3}$ [28].

In the first part of this chapter, the LAO/STO interface is studied with a focus on understanding the origin of the conductivity and its relation to the polar discontinuity. Out of the four models discussed in the previous paragraph, only the electronic reconstruction scenario takes the polar discontinuity into account. Therefore, experiments have been performed to test the validity of this model. The experiments unambiguously demonstrate that the model is not applicable to the experiments and that purely electronic reconstruction does not occur. It is concluded that the polar discontinuity results in a structural reconstruction, the details of which depend on sample geometry and fabrication conditions and affect the properties of the interface. The structural reconstruction results in a charge transfer of $0.5 e-/$ uc across the LAO.

In the second part of this chapter, an alternative model for the conductivity is proposed. One of the striking features of the LAO/STO interface is the thickness dependence of the conductivity $[3,4]$. This is explained qualitatively with the electronic reconstruction model. As this model is not applicable, an alternative explanation is required. Here, it is suggested that the conductivity is related to the induced polarization in the STO. Experimental results of the induced polarization as a function of LAO thickness, as measured with second harmonic generation (SHG), are compared with the activation energies for the charge carriers and a correlation is found. A model is proposed in which the polarization in the STO activates carriers from impurity states in the bandgap.

This chapter is organized as follows. First, an overview of relevant literature about LAO/STO interfaces is given. In the next section, experiments are presented which demonstrate the absence of the electrostatic potential buildup in the LAO layer. In section 4 , it is shown that insulating $n$-type interfaces can be fabricated, either by growing on other substrates than STO or by growing LAO at high $(0.1$ mbar) oxygen pressure. The experiments indicate that the intrinsic electronic reconstruction does not occur and therefore the polar discontinuity requires a structural reconstruction. Different scenarios are discussed in section 5 . In section 6 , the alternative model is proposed, relating the conductivity to the induced polarization in the STO. The chapter ends with an outlook for future experiments to test the validity of the proposed model. 


\subsection{Overview relevant literature}

A review of the transport properties can be found in [29], while a review of the theoretical understanding is given in [30]. In this section, both the theoretical understanding of and the experimental evidence regarding electronic reconstruction at the interface are briefly summarized.

\subsubsection{Theory}

The LAO/STO interface has been theoretically studied within the framework of density functional theory (DFT). This theory uses periodic boundary conditions and the object of study is always an infinite set of interfaces. The physics of the polar interfaces is governed by the electrostatic potential buildup. In DFT special care has to be taken with the geometry of the supercell, as potential buildup over the entire supercell is not allowed. Two approaches have been developed. The first is to use a supercell with $(k+1) \mathrm{LaO}$ and $k \mathrm{AlO}_{2}$ layers [31-38]. STO then has $m$ $\mathrm{SrO}$ and $(m+1) \mathrm{TiO}_{2}$ layers. In this case, the supercell has two $n$-type interfaces and there is no potential buildup due to symmetry. This approach automatically ensures the doping of 1 electron in the supercell. The doped charge accumulates at the $\mathrm{TiO}_{2}$ layers at the interface and results in a doping of $0.5 e-/$ uc. The same can be done with two $p$-type interfaces and in this case half a hole per unit cell is found in the oxygen band at the interfaces. The results can in principle be compared to the experimental case of a single interface with an extremely thick LAO layer if $k$ and $m$ are both reasonably large. These studies can give valuable information about the atomic and electronic structure of a fully reconstructed interface, but it cannot describe the potential buildup and the charge transfer, which is essential in the experiments [39].

The other approach is to include a vacuum space in the unit cell [39-42]. In this case both a $p$ and an $n$-type interface (or surface) can be present in the unit cell. There is potential buildup in the LAO layer and the charge transfer from e.g. the LAO surface (LAO/vacuum interface) to the $n$-type LAO/STO interface can be studied. As the electric potential must be periodic, the potential buildup in the LAO results in an equal potential drop over the combined vacuum and STO layers. This spurious field can be removed with either a dipole correction [42] or a doubled structure with a mirror plane in the vacuum [39]. This allows the DFT calculations to demonstrate the potential buildup in the polar layer and the subsequent electronic reconstruction. This approach uses a large supercell, which is computationally expensive, and therefore less structural relaxation is performed (typically only atomic shifts in $\mathrm{z}$ are allowed).

Sofar, the following points have been predicted by theory:

- A critical LAO thickness for conductivity is found [39, 40].

- Polarization is induced in the STO when charge transfer occurs [42]. 
- The electric field in the LAO layer induces structural displacements in the LAO (dielectric screening) [40].

- The reconstruction induces octahedra rotations $\left(\mathrm{GdFeO}_{3}\right.$ type) in the interfacial STO layer [35].

- The Ti conduction band splits in a heavy lower energy band which consists mostly of $3 \mathrm{~d}_{x y}$ states and a light higher energy band of mostly $3 \mathrm{~d}_{x z}$ and $3 \mathrm{~d}_{y z}$ states [37].

- At the $n$-type interface the STO conduction band is lowered due to chemical bonding between $\mathrm{Ti}$ and La and oxygen octahedra deformation, while the valence band is raised due to the induced polarization [39]. Electrons at the $n$-type interface are confined to the interface while holes at the $p$-type interface spread out due to the induced polarization [34].

- An alternative approach to calculate the polarization using Berry phase theory gives an elegant explanation for the fact that the polarization difference between LAO and STO is exactly $0.5 e-/$ uc [43].

- $n p$ superlattices have a different critical thickness due to the screening of the potential buildup in both the LAO and the STO in this configuration [24].

- Oxygen vacancies at the $p$-type LAO interface/surface are more energetically favourable than oxygen vacancies at the $n$-type interface [44-46].

\subsubsection{Experiment}

In order to understand the LAO/STO interfaces the following experimental observations have to be taken into account:

- A critical LAO thickness of $4 \mathrm{uc}$ is found for the conductivity [3].

- In coupled $n p$ interfaces, no critical thickness is observed. The room temperature carrier density depends on the interface separation distance [4].

- At the $p$-type interface oxygen vacancies are present and this interface shows less intermixing than the $n$-type interface [2].

- The room temperature carrier density as measured with transport measurements in well oxydized samples ranges from 0.015 to $0.3 e-/$ uc [3-5].

- The thickness of the electron gas at room temperature is less than $1 \mathrm{~nm}$ [47-49]. The thickness of the superconducting state at low temperature is $10 \mathrm{~nm}[50]$.

- The interfacial STO unit cell is elongated at the interface [51] but the oxygen octahedron is contracted [52].

- STO is polarized at the interface [53]. 
- $\mathrm{GdFeO}_{3}$ type oxygen octahedra rotations have been observed in the interfacial STO layers [53].

- The STO conduction band has mostly Ti $3 \mathrm{~d}_{x y}$ character [54].

- Different photoemission measurements do not agree on the band alignment $[55,56]$.

- La impurities are present in the STO [57, 58].

\subsection{Absence of potential buildup in the LAO}

The comparison between theory and experiment in section 2.2 shows that a number of observations are well understood. Nevertheless, some open issues remain. The electronic reconstruction scenario predicts that charge is transferred from the LAO valence band at the surface to the STO conduction band at the interface. Only the presence of charge at the interface is experimentally verified, namely the interface is conducting. This is not sufficient evidence for the charge transfer. It might well be possible that electrons due to impurities in the STO are accumulated at the interface. So to prove the mechanism of electronic reconstruction one of the following experiments should be done.

- A measurement of the electron density at the $n$-type interface and the hole density at the $p$-type interface which shows identical carrier densities for LAO films with different thicknesses.

- A measurement of the potential buildup in the LAO layer as a function of the LAO layer thickness. The total potential buildup should increase linearly with film thickness up to the critical thickness for electronic reconstruction. Then the potential buildup over the entire layer will be equal to the bandgap of the STO. The change in potential buildup per unit cell layer at the critical thickness directly reflects the electronic reconstruction.

The first experiment is difficult to perform with the LAO/STO interface as the hole density at the $p$-type interface is comprised of oxygen $2 \mathrm{p}$ states, which are difficult to analyze quantitatively using spectroscopic techniques. The second experiment can be performed using x-ray photoemission spectroscopy (XPS) measurements. The potential buildup in the LAO layer shifts all the LAO electron core levels and results in changes in the XPS spectra. In the actual experiment, no changes are observed and it is concluded that no electrostatic potential buildup is present at the LAO/STO interface.

\subsubsection{Experimental}

The samples were fabricated using pulsed laser deposition (PLD). Substrates were prepared using the standard preparation procedure described elsewhere [59]. 
Growth conditions were as follows: laser fluence $1.3 \mathrm{~J} / \mathrm{cm}^{2}$, oxygen pressure during growth $10^{-3} \mathrm{mbar}$, growth temperature $800^{\circ} \mathrm{C}$, growth rate $2.3 \mathrm{uc} / \mathrm{min}$. The samples were cooled down to room temperature at growth pressure and an additional post-growth annealing step was not applied. More information about the growth of LAO on STO can be found in reference [29]. The $\mathrm{La}_{0.67} \mathrm{Sr}_{0.33} \mathrm{MnO}_{3}$ (LSMO) reference sample was grown as described in section 3.3.

Reflection high energy electron diffraction (RHEED) specular spot intensity oscillations were observed during the growth, indicating 2D layer-by-layer growth. The film surface morphology was measured with AFM and the step and terrace structure of the underlying substrate was observed. Transport measurements of samples with a thickness of $n=5$ to $n=10$ uc of LAO showed conducting behaviour with a sheet resistance of $\approx 10^{4} \Omega$ at room temperature and $\approx 5 \cdot 10^{2} \Omega$ at $10 \mathrm{~K}$.

The XPS measurements were performed in situ with an XPS/UPS system designed by Omicron Nanotechnology GmbH, equipped with an EA 125 electron energy analyzer. For XPS an Al K $\alpha$ source (XM 1000) was used. The base pressure of the system was below $10^{-10}$ mbar. The analyzer was calibrated with the use of an in situ sputter cleaned Au sample.

\subsubsection{Measuring the potential buildup with XPS}

With XPS, the binding energy of electrons is measured in reference to the Fermi level. In an LAO film on STO with a polar interface, the binding energy of electrons in LAO changes with the potential buildup. As the potential buildup is gradual over the LAO thickness, the potential buildup will result in broadening of the peaks and also in a shift of the peak positions. To calculate the XPS peak shape of the LAO elements in the potential field, the LAO layers are separately analyzed and the contribution from each layer is calculated. The total signal is the sum of the layer contributions, in which an exponential decay of the intensity away from the surface is taken into account (escape depth $2 \mathrm{~nm}$ at $h v=1500 \mathrm{eV}$ [60]). As the conducting electron gas is in the STO at the interface, the LAO layer closest to the STO is pinned at the normal potential. A potential shift of 0.9 $\mathrm{eV} / \mathrm{uc}$ was used for LAO layers thinner than the critical thickness [25]. For LAO layers thicker than the critical thickness, the total potential buildup was set to 3.2 $e \mathrm{~V}$. The XPS peaks were modelled with Gaussian intensity distributions.

In figure 2.1, the calculated La $3 \mathrm{~d}$ peak shapes for a different number of LAO layers is shown ${ }^{1}$. It can be seen that the width of the peak shape is small for thin samples due to the small potential buildup and large for samples with intermediate thicknesses of 3-7 unit cell layer. For thick samples (with respect to the electron escape depth) the width is reduced again as then only a part of the layer is probed.

\footnotetext{
${ }^{1}$ The La 3d peaks are intrinsically quite wide, approximately $2.5 \mathrm{eV}$ full width at half maximum (FWHM). For the accuracy of the experiment, it would be better if the shape of the $\mathrm{Al} 2 \mathrm{p}$ peak $(1.2 \mathrm{eV}$ FWHM) was analyzed. This would result in a smaller error bar on the observed potential shift. Most of the available measurement data was of the La peaks, however, so these were used. The expected potential shift of $3.2 \mathrm{eV}$ is large enough to be observable with the La peaks.
} 


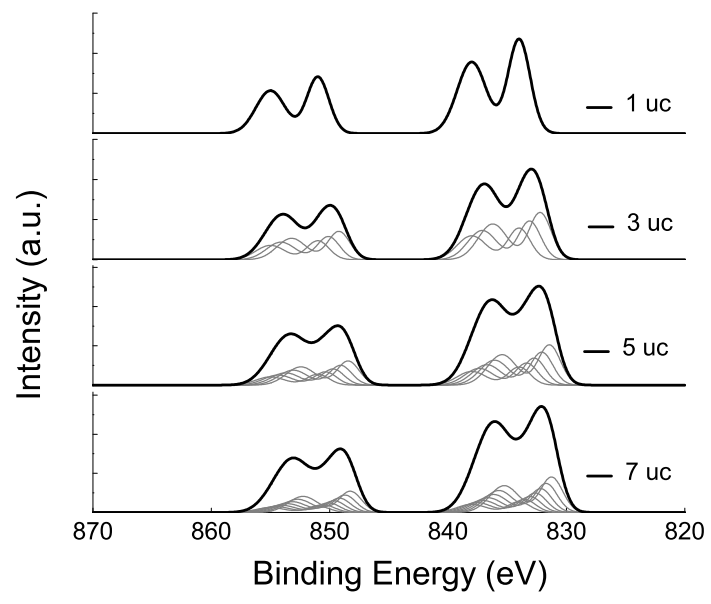

Figure 2.1: Simulation of the La 3d spectrum with the potential buildup due to the polar discontinuity at the $n$-type interface taken into account.

Table 2.1: La 3d peak width as indicated by FWHM. The error margin of the experimentally determined FWHM is $0.01 \mathrm{eV}$.

\begin{tabular}{ccccc}
\hline \hline Position $(e \mathrm{~V})$ & 834 & 839 & 851 & 856 \\
\hline Model 5uc & 2.94 & 3.99 & 2.94 & 3.99 \\
LAO/STO 5 uc & 2.36 & 2.75 & 2.0 & 2.62 \\
LSMO reference & 2.39 & 2.92 & 2.21 & 2.86 \\
\hline \hline
\end{tabular}

The other striking difference with the reference spectrum is the gradual shift of the peak onset towards lower binding energy. This shift is $3.2 \mathrm{eV}$ for samples thicker than the critical thickness.

For the experiment, the La $3 \mathrm{~d}$ peaks were measured for a set of $n$-type LAO/STO interfaces and LSMO reference samples. An equal peak width was observed for all samples as illustrated in figure 2.2. In this figure, a 5 unit cell layer LAO/STO interface is compared with a $20 \mathrm{~nm}$ thick LSMO reference sample. The LSMO is conducting, so here no broadening due to charging and potential shifts is expected. It is clear from the graphs that the LAO/STO sample does not show broader peaks compared to the LSMO sample. A shift of $1 \mathrm{eV}$ towards higher binding energy is observed, which is attributed to a material dependent difference in chemical shift. The spectra were fit with the use of Voigt functions. From the fit, the FWHM of the peaks were extracted as summarized in table 2.1. The same fit was used to determine the width of the calculated spectra in figure 2.1. The analysis of the peak width places an upper bound of $0.5 \mathrm{eV}$ on the potential buildup in the LAO layer. This is much less compared to the expected $3.2 \mathrm{eV}$. 


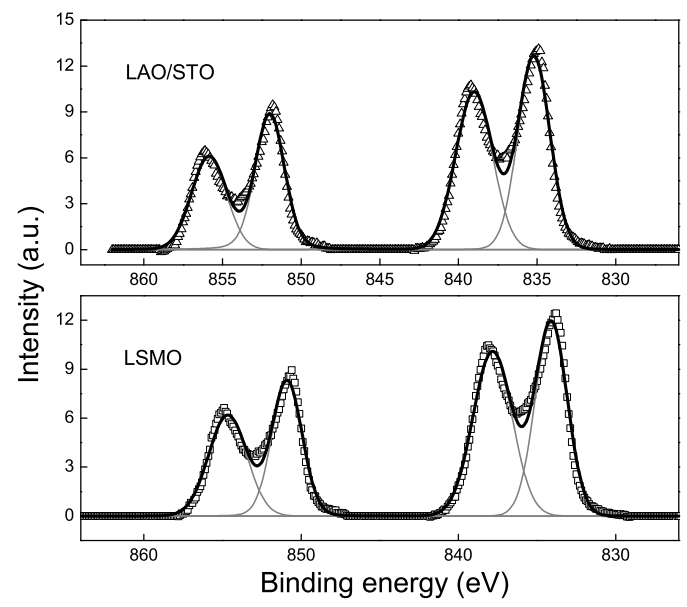

Figure 2.2: La 3d peak intensity after background subtraction. Top graph: a 5 unit cell LAO/STO sample (n-type interface). Bottom graph: a $20 \mathrm{~nm}$ LSMO sample.

\subsubsection{Summary}

The XPS experiments indicate that the potential buildup in the LAO layer is smaller than $0.5 \mathrm{eV}$, much smaller than the bandgap of STO. Therefore, the electronic reconstruction scenario for the conductivity at the LAO/STO interface is not applicable to the experiments. Similar results have been obtained by Segal et al. [56] for MBE grown samples. Finally, it is noted that the potential buildup is screened by atomic displacements $[40,61]$. Jia et al. have observed the atomic positions in the LAO layer using scanning transmission electron microscopy (STEM) [53]. The observed displacement is $0.00 \pm 0.02 \AA$, as opposed to the expected displacement of $0.11 \pm 0.04 \AA^{2}$. This also implies that no potential buildup is present in the LAO layer. Therefore, it is concluded that the polar discontinuity is reconstructed by means of a structural reconstruction. This reconstruction results in a charge transfer of $0.5 e-/$ uc across the LAO.

\section{$2.4 \quad$ Insulating $n$-type interfaces}

In the previous section, it was concluded that the polar discontinuity does not result in intrinsic electronic reconstruction but in a structural reconstruction. This

\footnotetext{
${ }^{2}$ The expected displacement is based on two sets of DFT calculations. Pentcheva et al. mentions a displacement of $0.15 \pm 0.05 \AA$ for a five layer sample [40]. Assuming a linear displacement with applied electric field this gives a displacement of $0.11 \pm 0.04 \AA$ for the 7 unit cell layer sample measured by Jia et al.. Also Son et al. calculated the ionic displacements for a 7 layer LAO sample [42, 62]. They found a displacement of $0.1 \AA$.
} 
structural reconstruction does not necessarily result in conducting samples, as is shown in this section. First, it is shown that LAO/STO interfaces grown on other substrates than STO are insulating, which is attributed to the difference in strain in the STO layer. In the second part, it is shown that LAO/STO interface grown at high oxygen pressure (0.1 mbar) are insulating as well.

\subsubsection{LAO/STO interfaces on $\mathrm{NdGaO}_{3}$ substrates}

$N$-type LAO/STO interfaces were grown on (110) o $\mathrm{NdGaO}_{3}$ (NGO) substrates. (The subscript o denotes the orthorhombic crystal structure. The (110)o surface corresponds to a pseudocubic (001) surface.) Two types of samples were grown. The first type consisted of first 10 unit cell layers of STO and then 10 unit cell layers of LAO. As NGO is expected to be $B$-site terminated, this structure is expected to be $n$-type and conducting. The other type of sample is made by first growing 10 unit cell layers of LAO, then 1 unit cell layer of $\mathrm{LaTiO}_{3}$ (LTO) and finally 10 unit cell layers of STO. This also results in an $n$-type LAO/STO interface.

\section{The NGO substrate}

NGO has a lattice constant in pseudocubic units of $3.86 \AA$, which is in between the lattice constants of STO and LAO. So an STO film on NGO is under compressive strain while an LAO film is under tensile strain. The NGO surface can be singly terminated with a combination of a chemical treatment and an anneal treatment as shown elsewhere [63]. It was not mentioned whether the termination after the treatment is $A$-site $(\mathrm{NgO})$ or $B$-site $\left(\mathrm{GaO}_{2}\right)$. In order to obtain information about the termination, $\mathrm{SrRuO}_{3}$ (SRO) was grown on a treated substrate. The initial growth of $\mathrm{SRO}$ is sensitive to $A$ - or $B$-type termination, as the $\mathrm{RuO}_{2}$ surface is unstable. When SRO is grown on a $B$-site terminated surface, a termination conversion occurs within the first few unit cell layers and this can be observed by comparing the periods of the RHEED oscillations [64]. The intensity of the specular spot during the RHEED monitoring of SRO growth on NGO is shown in figure 2.3. The PLD parameters for the growth were $700{ }^{\circ} \mathrm{C}, 0.05$ mbar oxygen and a laser fluence of $2 \mathrm{~J} / \mathrm{cm}^{2}$. The longer period of the first oscillation indicates growth on a $B$-site terminated surface. So it is expected that the NGO substrate surface after treatment is $\mathrm{GaO}_{2}$ terminated.

\section{Growth and characterization}

In this section, the growth and characterization of LAO/STO interfaces on NGO will be described. Both STO and LAO were grown with the standard PLD settings for the growth on STO substrates [29]. The most important parameters were an energy density of $1.3 \mathrm{~J} / \mathrm{cm}^{2}$, a substrate temperature of $800{ }^{\circ} \mathrm{C}$ and an oxygen pressure of $10^{-3}$ mbar. Both materials grow layer-by-layer as was evidenced by 


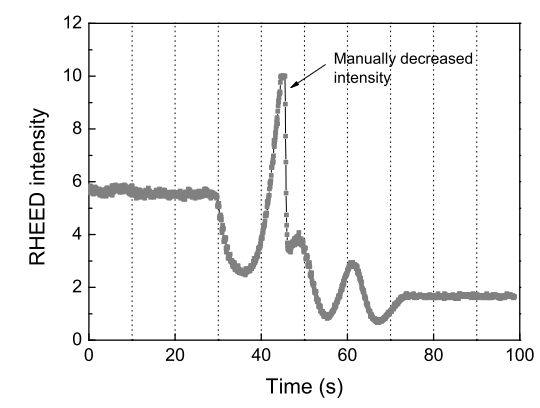

Figure 2.3: RHEED intensity of SRO growth on NGO. The first oscillation is prolonged with respect to the second oscillation, indicating a termination conversion [64].

analyzing the RHEED specular spot intensity. The surface topography was investigated with atomic force microscopy (AFM). A topography map of an LAO/STO structure on NGO is shown in figure 2.4. The step and terrace structure of the underlying substrate is clearly visible. Lateral force microscopy did not show any contrast, which indicates a singly terminated surface. X-ray diffraction (XRD) measurements were used to determine both in-plane and out-of-plane lattice parameters. It was found that both materials are coherently strained with identical in-plane lattice parameters to the NGO substrate. The out-of-plane lattice parameters of STO respectively LAO on NGO were 3.95 and $3.73 \AA$.

Electrical contact was made to the sample with $\mathrm{Al}$ wire bonds. The 10 unit cell layer LAO on 10 unit cell layer STO on NGO sample was found to be insulating. A sample with the reverse stacking was found to be insulating as well and also samples fabricated at an oxygen partial pressure of $10^{-5}$ mbar. Similar structures grown on $(110)_{\mathrm{o}} \mathrm{DyScO}_{3}$ (DSO) substrates were insulating. Finally the other type of stacking (first $10 \mathrm{LAO}$, then 1 LTO and 10 STO) also resulted in insulating samples.

\subsubsection{LAO/STO interfaces grown in high oxygen pressure}

In the preceding section, it is shown that LAO/STO interfaces grown on NGO substrates are insulating. In this section, the properties of LAO/STO interfaces on STO substrates are analyzed with respect to the oxygen pressure during growth. The previous study which related the properties of the interface to oxygen pressure [8] did not report experiments up to 0.1 mbar where the expected amount of oxygen vacancies is minimal.

The samples were fabricated using pulsed laser deposition (PLD), as discussed in section 2.3.1. Samples were grown with an LAO thickness of 2 and 6 unit cells. Three different oxygen partial pressures were used: $10^{-1}$ mbar (high pressure: 


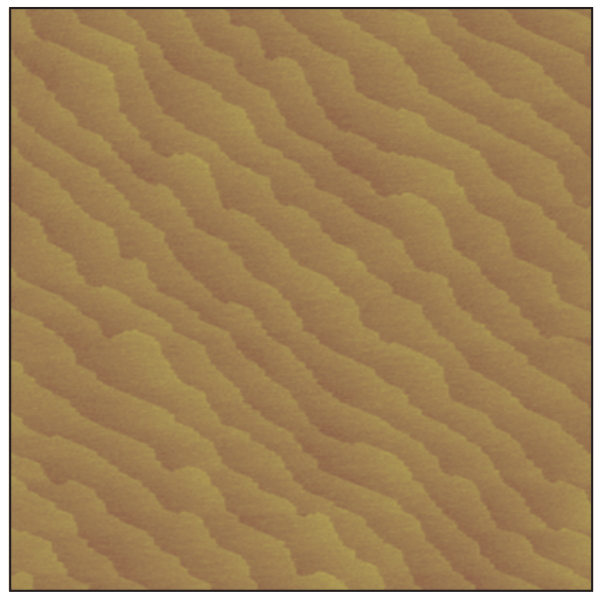

Figure 2.4: AFM topography measurement of an LAO/STO/NGO sample with 10 unit cell layer LAO and STO. The image is 5 by $5 \mu \mathrm{m}$ and the height differences between the terraces correspond to a single perovskite unit cell $(\approx 4 \AA)$.

HP), $10^{-3}$ mbar (medium pressure: MP), and $10^{-5}$ mbar (low pressure: LP). The samples were cooled down to room temperature at growth pressure and an additional post-growth annealing step was not applied.

Conductivity was measured ex situ using the van der Pauw geometry [65]. Results are plotted in figure 2.5 and show a strong dependency on the growth pressure. Independent of the overlayer thickness, both LP samples (lower, dark green and middle, light green curve) exhibit clear metallic behaviour with a very high conductivity. Turning to the MP regime, only the 6 uc samples (amber curve) show conducting behaviour. The 2 uc MP samples remain insulating down to the lowest temperatures with a resistivity $>20 \mathrm{M} \Omega$, as expected from the critical thickness for conductivity.

In contrast to the LP and MP samples, both HP samples are insulating. Maurice et al. also found samples to be insulating, if grown at 0.4 mbar [66]. This was explained by structural defects in the film. In contrast, the HP samples in this study did not show an enhanced defect or dislocation density, neither at the interface nor in the bulk film. This was confirmed by scanning transmission electron microscopy (STEM), an example of which is shown in figure 2.6a. The samples exhibit the same atomically sharp interface compared to the MP and LP samples (not shown). This was also confirmed by AFM topography measurements, one of which is displayed in figure $2.6 \mathrm{~b}$. The surface is atomically smooth with single step terraces. Compared to the samples of Maurice et al., an increased surface roughness was not observed [66]. On the other hand, it was observed that the RHEED oscillations were not as clear as for the growth of the LP and MP samples. This indicates a reduced surface diffusion during growth. 


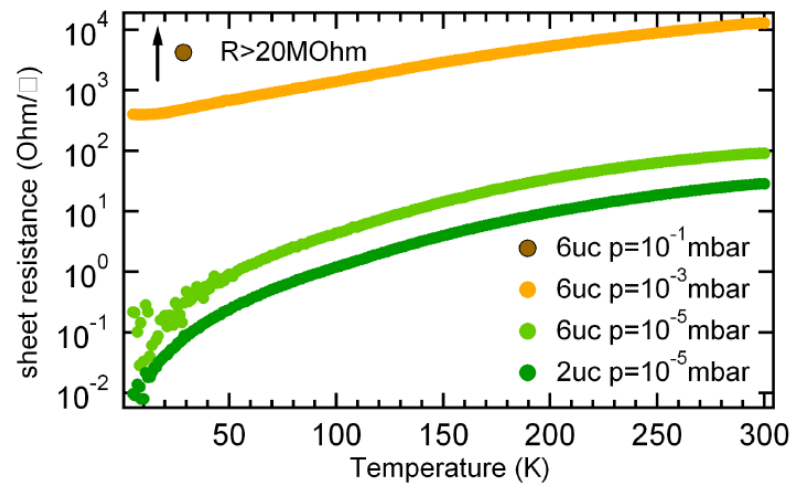

Figure 2.5: Resistivity of the LAO/STO interfaces grown at $10^{-5}, 10^{-3}$ and $10^{-1}$ mbar.
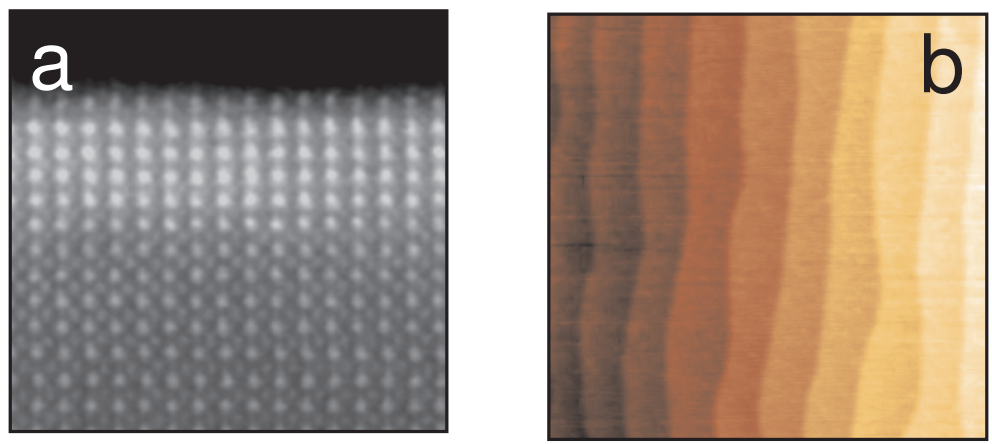

Figure 2.6: Characterization of a 6 unit cell LAO on STO sample grown at $10^{-1}$ mbar. a) STEM and b) AFM. The AFM image is 2 by $2 \mu \mathrm{m}$ and the height differences between the terraces correspond to a single perovskite unit cell $(\approx 4 \AA)$. The STEM image was obtained at the University of Würzburg, Germany. 


\subsubsection{Conclusions}

High quality LAO/STO heterostructures were realized on NGO substrates. These interfaces are insulating. Also the oxygen pressure dependence during LAO growth on STO substrates was investigated. The results show that high pressure samples are insulating, intermediate pressure samples are conducting with the expected critical LAO thickness and low pressure samples are very conductive without a critical LAO thickness.

\subsection{Structural reconstruction of the polar discon- tinuity}

The experiments discussed in the previous section cannot be explained in the framework of the polar discontinuity induced potential buildup and electronic reconstruction. This implies that other reconstruction mechanisms for the polar discontinuity at the interface have to be considered. In this section several possible mechanisms are discussed.

The polar discontinuity is reconstructed by the transfer of a sheet charge of $0.5 e$ /uc from the $p$-type surface (or interface) to the $n$-type interface (or surface). For simplicity only the case of a single LAO film on STO, i.e. a single $n$-type interface and a $p$-type surface, is considered. The possibilities for the reconstruction of the $p$-type surface are listed below:

- Al-Ti substitution, top layer is $\mathrm{Al}_{0.5} \mathrm{Ti}_{0.5} \mathrm{O}_{2}$.

- Ionized adsorbates.

- Oxygen vacancies in the $\mathrm{AlO}_{2}$ layer, $\frac{1}{4} \mathrm{~V}^{+} / \mathrm{uc}$.

- Additional La ions on the $p$-type surface layer, $\frac{1}{6}$ ion/uc.

and the $n$-type interface:

- Ti-Al substitution, interfacial STO layer is $\mathrm{Al}_{0.5} \mathrm{Ti}_{0.5} \mathrm{O}_{2}$.

- Charge in the STO, $0.5 \mathrm{e}$-/uc.

- La ion vacancies in the first LaO layer, $\frac{1}{6}$ ion/uc.

The Ti-Al substitution is highly unlikely due to the low diffusivity of $B$-site ions in perovskites. Moreover, STEM studies have observed sharp interfaces on the $B$-site lattice with minimal Ti-Al intermixing $[2,4]$. Also the chemical reconstruction with ionized adsorbates is unlikely. The samples are grown in an ultra-high vacuum system and the reconstruction has to occur before the samples are exposed to outside influences. Combining the reconstructions for the interface and the surface, two scenarios are left: oxygen vacancies are present at the LAO surface 
and donate electrons into the STO or La ions diffuse through the LAO layer and create vacancies at the interface and adatoms at the surface.

To explain the growth pressure dependence, it is suggested that the growth pressure influences the type of reconstruction. When LAO is grown at low or intermediate pressure, oxygen vacancies are present in the material and a sufficient amount of vacancies accumulate at the surface to compensate the polar discontinuity. During the cooldown, the vacancies in the $\mathrm{AlO}_{2}$ surface layer remain. Therefore, charge is present in the STO in these samples. When LAO is grown at high oxygen pressure, insufficient oxygen vacancies are present and La ions will diffuse towards the surface. This explains why the samples grown at high oxygen pressure are insulating.

Finally, it is noted that the two structural mechanisms discussed in this section can be combined in a two-step reconstruction. In that case, charge is transferred from oxygen vacancy sites at the LAO surface to the LAO/STO interface. At the interface, La vacancies are present $\left(\frac{1}{6}\right.$ ion/uc). As these missing La ions have to go somewhere, they diffuse into the STO. This implies Sr vacancy sites are already present in the substrate or Sr ions will diffuse. The La in the STO then dopes the STO. In this case, only $\frac{1}{6} e-/$ uc of charge is present in the STO and this scenario also explains the observation of La ions in the STO substrate.

\subsection{Thickness dependence of the conductivity and induced polarization in the STO}

In the first part of this chapter, it was shown that the polar discontinuity at the LAO/STO interface is compensated with a structural reconstruction, which results in a charge transfer of $0.5 \mathrm{e}$-/uc across the LAO. It is reasonable to assume that the type of structural reconstruction depends on the growth parameters, such as the oxygen pressure during growth. The absence of conductivity in the high pressure samples can thus be explained by assuming a different structural reconstruction, e.g. one not involving surface oxygen vacancies, in these samples. It is unlikely, however, that the structural reconstructions depend dramatically on the strain state of the STO. The insulating interfaces on NGO substrates were grown at low oxygen pressure as well and remained insulating. More importantly, the critical thickness for the conductivity as observed by Thiel et al. [3] and the coupling of $n$ - and $p$-type interfaces as observed by Huijben et al. [4] show that the conductivity depends on the LAO layer thickness. This thickness dependence was originally interpreted as an indication for the validity of the intrinsic electronic reconstruction model, but as there is no electrostatic potential buildup in the samples, due to the structural reconstruction, this explanation is no longer applicable. It is unlikely that the structural reconstruction of the polar discontinuity has a thickness dependence, so an alternative model is required.

Here, an alternative model is presented, which relates the conductivity of the LAO/STO interface to the induced polarization in the STO. Experiments have 
shown that polarization is induced in the STO at the LAO/STO interface [53]. This induced polarization is discussed in the first part of this section. In the next part, the thermal activation of the sheet carrier density in conducting LAO/STO samples is discussed and it is shown that the activation energy has a similar trend as the induced polarization in the samples. Finally, a model is proposed which relates the strain induced polarization to the conductivity of the LAO/STO interfaces.

\subsubsection{Induced polarization}

Here, the induced polarization in the STO is discussed, both from a theoretical, as well as from an experimental point of view. In the theoretical part, it is shown that spontaneous polarization is present at the interface. The main observation discussed in the experimental part is that the polarization is not induced by the charge transfer. It is suggested that the polarization is related to strain of the oxygen octahedra rotation discontinuity.

\section{Theoretical point of view}

In an electrostatic model of the LAO/STO interface, the polar discontinuity at the interface does not induce polarization in the STO until charge transfer occurs. When charge is transferred to the STO layer, the charge spreads throughout the STO and an electric field is present between the charge in the STO and the charge of opposite sign at the LAO surface. This field induces polarization. Similar behaviour, coincidence of charge transfer and induced polarization, is observed in DFT calculations as well [42].

There is, however, a significant difference in the band bending in the STO between the electrostatic model and the DFT calculations. In the dipole model, the band bending is such that charge carriers (electrons (holes) in the STO at the $n$-type ( $p$ type) interface) are confined to the interface, as shown in figure 2.7. Note that here, the band bending is due to the electric field between the charge carriers and the donor surface states, which is partially screened by the induced polarization in the STO. In the DFT calculations the band bending for the $p$-type interface is opposite to that of the dipole model [41]. The sign of the polarization in the STO, however, is equal to that of the dipole model. From this is it concluded that the STO overscreens the electric field in the DFT calculations. For the $n$-type interface, bandgap narrowing is calculated. The valence band bends in the opposite direction compared to the dipole model but the conduction band bending is similar. The valence band bending is due to the induced polarization while the conduction band bending is dominated by hybridization of the interfacial Ti and La wavefunctions [39]. The overscreening of the electric field by the STO is unexpected but not impossible. STO is a quantum paraelectric at low temperatures whose spontaneous polarization is completely reduced by quantum fluctuations [67]. In the presence of a small field these fluctuations are suppressed and spontaneous polarization can be induced. 

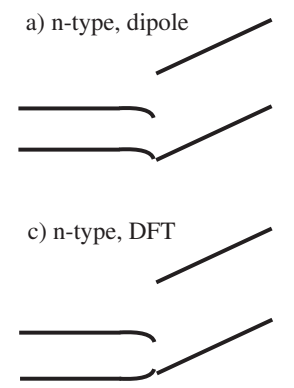

e) n-type, fields

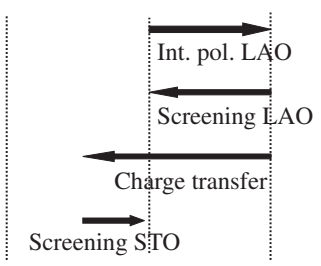

b) p-type, dipole

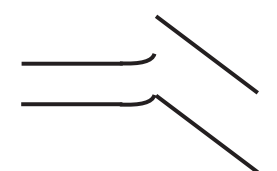

d) p-type, DFT

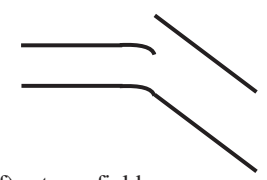

f) p-type, fields

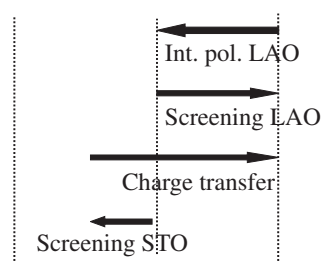

Figure 2.7: A sketch of the band bending in the STO at the LAO/STO interface, STO (LAO) is shown on the left (right) side of each image. For the $n$-type interface (left images), the LAO valence band at the surface is equal in energy to the STO conduction band at the interface, while for the $p$-type interface (right image), the STO valence band at the interface is equal in energy to the LAO conduction band at the surface. The band bending in the STO has been exaggerated. a) and b) The interfaces as calculated by the dipole model. c) and d) The interfaces as calculated by the DFT [41]. e) and f) Arrows indicate all the electric fields in the structure: the intrinsic polarization in the LAO, the dielectric screening in the LAO, the charge transfer in the reconstruction and the dielectric screening of the charge transfer in the STO. 


\section{Experimental point of view}

The induced polarization of the STO at the LAO/STO interface was measured with STEM in a 7 uc thick LAO layer sandwiched between STO layers. Figure 3 in the paper by Jia et al. [53] shows the positions of the oxygen anions with respect to the $B$ cations. Polarization is present in the STO in a layer of approximately 4 uc thickness located at the interface. Using the measured displacement and the ionic charge of $6 \mathrm{e} / \mathrm{uc}$, an estimate of the polarization of $8 \mu \mathrm{C} / \mathrm{cm}^{2}$ can be made. It is interesting to note that the polarization direction is similar for both the $n$ - and the $p$-type interface (in both cases the negative $\mathrm{O}$ ions are displaced towards the LAO). From theory, the expected sign of the polarization depends on the charge transfer and therefore should be opposite for the two different atomic stacking sequences. This is an indication that the polarization is not induced by the charge transfer.

Optical second harmonic generation (SHG) is another tool to study the polarization in the LAO/STO samples. Second harmonic light is only generated by materials without inversion symmetry and the intensity of this light reflects the polarization. Two papers describe measurements of the SHG intensity of LAO/STO interfaces. Savoia et al. measured the SHG signal of single $n$-type LAO/STO interfaces with different LAO thicknesses [68]. Ogawa et al. measured the SHG intensity of LAO/STO superlattices (both $n$ - and $p$-type interfaces) for different sublayer thicknesses [69]. Both papers ascribe the measured signal to the STO, in the case of Savoia to the polarizability of the free electrons at the interface and in the case of Ogawa to the lattice polarization of the STO. As it is known from STEM measurements that polarization is induced in the STO [53], it is likely that the signal measured by Savoia is due to the STO polarization as well and not due to the free electrons.

In figure 2.8a, the SHG intensity for the single LAO/STO interfaces is presented [68]. There is large scatter in the signal for the three unit cell layer samples and for thicker samples significant SHG intensity is measured. For the 1 and 2 unit cell layer samples, the measured SHG sample is equal to or smaller than the baseline of the intensity obtained from a bare STO substrate. In figure $2.8 \mathrm{~b}$, the data obtained from the superlattices is presented [69]. The SHG intensity increases almost linearly with the STO sublayer thickness $n$ up to $n=8$, after which it decreases. The decrease of the intensity for $n>8$ corresponds well with the 4 unit cells of polarized STO observed with the STEM experiment; the polarization is limited to $4 \mathrm{uc}$ at both interfaces and therefore the volume polarization decreases for $n>8$. The linear increase observed for $n<8$ implies that the polarization in the superlattice samples also increases linearly with thickness.

The reconstruction of the polar discontinuities is very different for the superlattices compared to the single interfaces. Due to the multiple interfaces present, a global potential buildup is not possible. In theory, this results in a zigzag potential between the STO and the LAO [24] with a much larger critical thickness for electronic reconstruction than $4 \mathrm{uc}$. The zigzag potential implies the STO is 

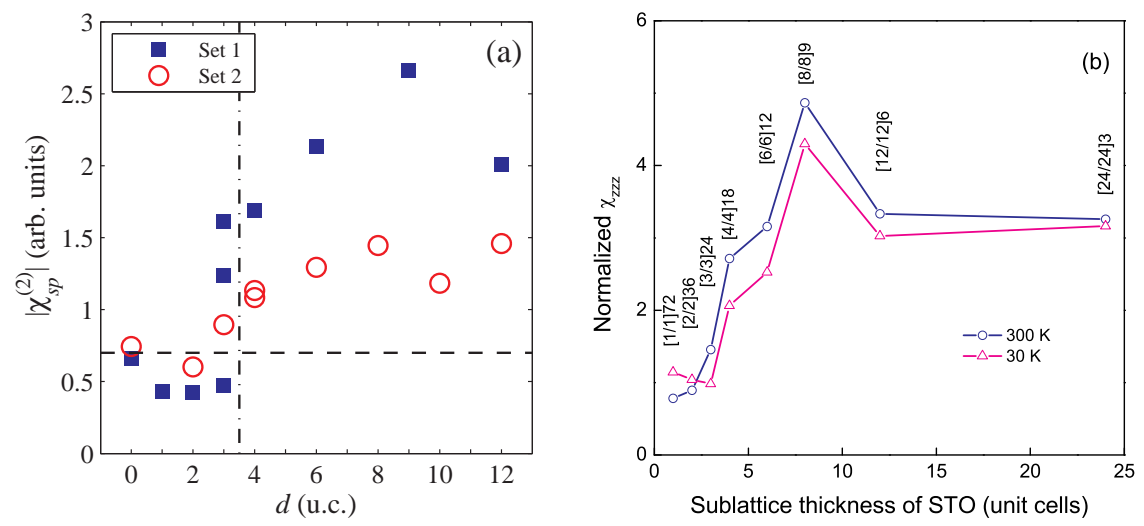

Figure 2.8: SHG intensity measurements. a) single interfaces: the $s p$ oriented part of the dataset measured by Savoia et al. [68]. b) $(n, m)$ superlattices of STO (sublayer thickness $n$ unit cells) LAO (sublayer thickness m unit cells) as measured by Ogawa [69]. These figures have been reproduced with permission from the authors. (c) 2009 the American Physical Society.

polarized regardless of the thickness up to the critical thickness ${ }^{3}$. The polarization is then simply $0.25 \mathrm{e}-/ \mathrm{uc}$, or $25 \mu \mathrm{C} / \mathrm{cm}^{2}$. The constant polarization of the STO with thickness is clearly not observed in the SHG experiments. This implies all LAO layers in the stack are individually reconstructed and no potential buildup or zigzag potential is present is the superlattice samples either. As charge transfer to compensate the polar discontinuity is present for all thicknesses, it is concluded that the polarization in the STO is not induced by charge transfer.

\section{Relation to oxygen octahedra rotations}

As an alternative to polarization induced by charge transfer, the relation between polarization and the discontinuity in the oxygen octahedra rotations at the interface is explored. STO is characterized by a cubic/tetragonal lattice without significant rotations, while LAO is rhombohedral (in bulk) with significant rotations. Under tensile strain LAO is expected to have rotations around the $[100]_{\mathrm{pc}}$ and $[010]_{\mathrm{pc}}$ axes, as observed experimentally [53]. (The subscript pc denotes the pseudocubic lattice.) At the interface a discontinuity exists and as the oxygen sublattice is coupled, reconstructions involving octahedra deformations must occur. These reconstructions can take place within the STO or within the LAO and have a characteristic lengthscale of a few unit cells [71]. As STO is easily deformed structurally, it can be expected that the oxygen octahedra deformations take place within the STO; this also implies some octahedra rotation is induced in the STO.

\footnotetext{
${ }^{3} \mathrm{~A}$ critical thickness 8-12 uc was mentioned by the Bristowe. This value is based on an estimate of the dielectric constant of STO of 30. Using a more realistic value of the dielectric constant of 300 [70], a critical thickness of $45 \mathrm{uc}$ is found.
} 
The rotations of the octahedra in the STO have been predicted [35], as well as measured [53].

Now a connection between the octahedra structure and the polarization has to be made. The relation between polarization and oxygen octahedra rotation and deformation is not well studied. One recent paper discusses the relation between polarization and oxygen octahedra rotation around the axis of polarization [72]. Although it was found that the polarization and the rotation are related, it does not apply to the case of LAO/STO where rotations around the $[100]_{\mathrm{pc}}$ and $[010]_{\mathrm{pc}}$ axes are induced and the polarization is in the $[001]_{\mathrm{pc}}$ direction. Moreover, the effect of oxygen octahedra deformation is not discussed. The deformations break the local symmetry of the Ti cations within the octahedra. Therefore, it is suggested here that the deformations of the oxygen octahedra in the STO, induced by the discontinuity of the oxygen octahedra rotations between LAO and STO, enable spontaneous polarization in the STO.

With these assumptions, the LAO thickness dependence of the polarization can be explained. The oxygen octahedra rotations discontinuity only exists when the rotation pattern in the LAO is developed, which occurs at a critical thickness. This is the result of an energy balance between bulk LAO layers which favour rotations, and the interfacial and surface LAO layer which favour absence of rotation. For the single interface samples, polarization is found at $n>3 \mathrm{uc}$, while for the superlattice samples, polarization is found for all $n$. This difference can be explained if the energy cost of inducing rotations at an interface with STO is smaller than that of inducing rotations at the LAO surface reconstruction.

\subsubsection{Thermally activated conductivity}

Huijben et al. presented the LAO thickness dependence of the conductivity of coupled $n$ - and $p$-type interfaces. A linear increase in room temperature sheet carrier density with thickness was observed for $n<6$. The low temperature sheet carrier density, however, was independent of the LAO thickness at $2 \cdot 10^{13}$ $\mathrm{cm}^{-2}$. This suggests a difference in thermally activated transport between the samples. An Arrhenius plot of the sheet carrier density of these samples is shown in figure 2.9. Note that these samples were grown at low pressure (LP, $10^{-5}$ mbar). Two different temperature regions of thermal activation are identified with different activation energies [27]. At intermediate temperatures the activation energy is approximately $6 \mathrm{meV}$, independent of the sample thickness. The low temperature $(<20 \mathrm{~K})$ activation energy depends on the LAO thickness and ranges from $9 \mathrm{meV}$ for the $n=1$ sample to $4 \mathrm{meV}$ for the $n=5$ sample.

The thickness dependence of the low temperature activation energy is presented in figure 2.10. Next to the LP samples already discussed, data obtained from samples grown at an intermediate pressure (MP, $10^{-3} \mathrm{mbar}$ ) is shown as well. The general properties of the MP samples are discussed in detail in reference [25]. A similar trend in activation energy compared to the LP samples is observed. The activation energy decreases with thickness from $15 \mathrm{meV}$ to $5 \mathrm{meV}$. The difference in activation energies between the LP and MP samples is attributed to a difference in the energy 


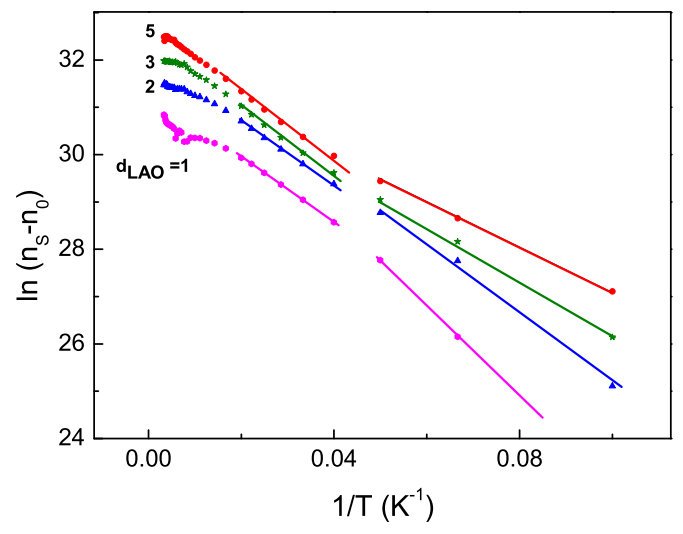

Figure 2.9: Arrhenius plot of the sheet carrier density of coupled LAO/STO interfaces. Two activation energies can be discriminated, one for the low temperature region and one for the intermediate temperature region. The lines are guides to the eye to illustrate the trends in the activation energy. This dataset was obtained from samples grown at $10^{-5}$ mbar and discussed in reference [4].

of the impurity levels, due to details of the sample fabrication. Nevertheless, the main trend of decreasing activation energy with increasing LAO thickness is the same.

The two different activation energies in the LAO/STO samples can be interpreted as follows. Different impurity states are present in the STO at different energies. As only the low temperature activation energy depends on the LAO thickness, it is suggested that this activation energy corresponds to impurity states close to the interface. The thickness independent activation energy of $6 \mathrm{meV}$ then corresponds to the bulk STO impurity level. An activation energy of $6 \mathrm{meV}$ was observed in La-doped STO [73], which agrees well with this interpretation.

\subsubsection{The complete picture: polarization induced conducti- vity}

Comparing the behaviour of the polarization in the superlattices and the charge carrier activation energy for the coupled interface samples, a similar linear increase with thickness is observed. The appearance of conductivity at a critical thickness of 4 uc [3] for single interface samples also follows the appearance of the STO lattice polarization, figure 2.8a. As shown in this figure, there is almost no polarization in the STO for LAO overlayers of 1 and $2 \mathrm{uc}$, intermediate polarization for the $3 \mathrm{uc}$ sample and a well developed polarization for the thicker LAO layer samples. The intermediate polarization of the 3 uc sample coincides with the thickness at which 


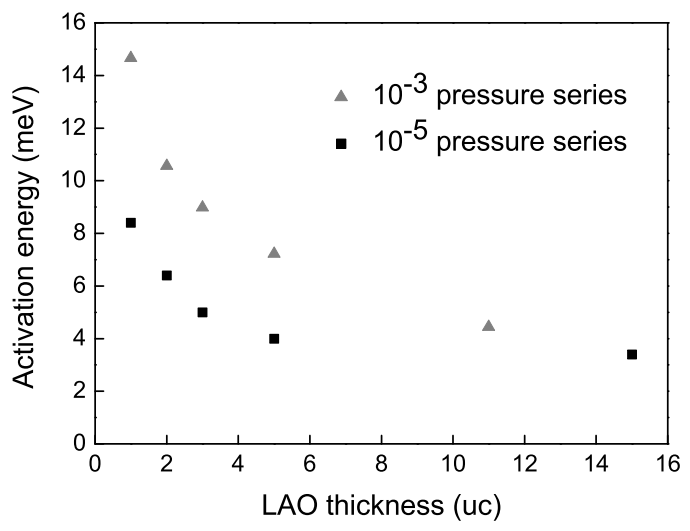

Figure 2.10: The low temperature activation energy as a function of LAO thickness for coupled LAO/STO interfaces. This dataset was obtained from samples grown at $10^{-5} \mathrm{mbar}(\mathrm{LP})$ and discussed in reference [4] and samples grown at $10^{-3}$ mbar (MP) and discussed in reference [25]. The LP datapoint at $n=15$ is actually a single interface sample instead of a coupled interface.

conductivity can be induced with a gate voltage. As discussed in section 2.6.1, the polarization in the STO results in band bending, which increases the energy of states at the interface. Therefore, it is suggested that the induced polarization increases the energy of the impurity states within the bandgap and conductivity occurs when electrons can move from the impurity states into the STO conduction band deeper in the substrate. This process is schematically presented in figure 2.11.

This model for conductivity at the LAO/STO interface has the benefits that it explains both the thickness dependence, as well as the absence of electrostatic potential buildup. Moreover, the polarization induced conductivity also explains the lack of conductivity of LAO/STO grown on NGO substrates, where the spontaneous polarization in the STO at the LAO/STO interface is expected to be suppressed, due to the strain in the STO layer.

\subsection{Conclusions and outlook}

In this chapter, the conductivity at the LAO/STO interface was studied. The main experimental result is that the expected electrostatic potential buildup in the LAO layer is not observed. Furthermore, samples grown on other substrates than STO and samples grown at high oxygen pressure are insulating. The results indicate that the most widely used model to explain the conductivity at the interface, the electronic reconstruction due to the polar discontinuity, is not applicable to the experiments. An alternative model for the conductivity is proposed, in which the 
a)

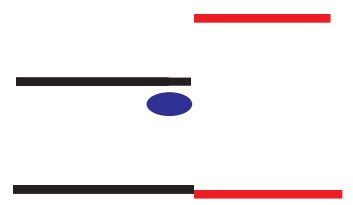

b)

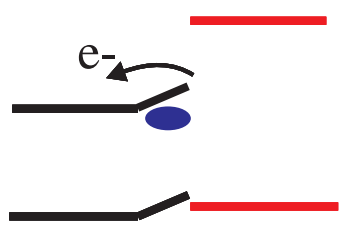

Figure 2.11: Schematic of the proposed LAO/STO band structure a) without polarization in the STO and therefore no conductivity and b) with polarization in the STO and therefore conductivity and thermally activated transport. The STO (LAO) bands are indicated in black (red) and the impurity states in blue. Electron transfer is indicated as well. The polarization in the STO results in band bending.

polar discontinuity is compensated by structural reconstructions, details of which depend on the growth conditions. At certain growth conditions, the structural reconstructions result in charge transfer to the STO and the charge in the STO resides in impurity states within the bandgap. Conductivity occurs when the induced polarization in the STO activates the charge carriers to the conduction band. As the induced polarization is related to the strain of the oxygen octahedra rotation discontinuity at the interface, the thickness dependence of the conductivity is explained.

The following experiments and calculations are suggested to further resolve the origin of the conductivity at the LAO/STO interface:

- More advanced structural characterization of both conducting and insulating samples. The structural reconstructions which compensate the polar discontinuity must be observed in order to understand the polar LAO/STO interface.

- SHG measurements of LAO/STO superlattices grown on NGO to search for (lack of) induced polarization.

- DFT studies of LAO/STO interfaces without a polar discontinuity (e.g. by including a $\mathrm{La}_{0.5} \mathrm{Sr}_{0.5} \mathrm{O}$ layer) to search for the oxygen octahedra rotations and induced polarization as a function of LAO thickness. 


\subsection{References}

[1] A. Ohtomo and H. Hwang, "A high-mobility electron gas at the $\mathrm{LaAlO}_{3} / \mathrm{SrTiO}_{3}$ heterointerface," NATURE, vol. 427, pp. 423-426, 2004.

[2] N. Nakagawa, H. Hwang, and D. Muller, "Why some interfaces cannot be sharp," NATURE MATERIALS, vol. 5, pp. 204-209, 2006.

[3] S. Thiel, G. Hammerl, A. Schmehl, C. W. Schneider, and J. Mannhart, "Tunable quasi-two-dimensional electron gases in oxide heterostructures," SCIENCE, vol. 313, pp. 1942-1945, 2006.

[4] M. Huijben, G. Rijnders, D. Blank, S. Bals, S. Van Aert, J. Verbeeck, G. Van Tendeloo, A. Brinkman, and H. Hilgenkamp, "Electronically coupled complementary interfaces between perovskite band insulators," NATURE MATERIALS, vol. 5, pp. 556-560, 2006.

[5] W. Siemons, G. Koster, H. Yamamoto, W. A. Harrison, G. Lucovsky, T. H. Geballe, D. H. A. Blank, and M. R. Beasley, "Origin of charge density at $\mathrm{LaAlO}_{3}$ on $\mathrm{SrTiO}_{3}$ heterointerfaces: Possibility of intrinsic doping," PHYSICAL REVIEW LETTERS, vol. 98, p. 196802, 2007.

[6] W. Siemons, G. Koster, H. Yamamoto, T. H. Geballe, D. H. A. Blank, and M. R. Beasley, "Experimental investigation of electronic properties of buried heterointerfaces of $\mathrm{LaAlO}_{3}$ on $\mathrm{SrTiO}_{3}, "$ PHYSICAL REVIEW B, vol. 76, p. $155111,2007$.

[7] A. Kalabukhov, R. Gunnarsson, J. Borjesson, E. Olsson, T. Claeson, and D. Winkler, "Effect of oxygen vacancies in the $\mathrm{SrTiO}_{3}$ substrate on the electrical properties of the $\mathrm{LaAlO}_{3} / \mathrm{SrTiO}_{3}$ interface," PHYSICAL REVIEW B, vol. 75, p. $121404,2007$.

[8] A. Brinkman, M. Huijben, M. Van Zalk, J. Huijben, U. Zeitler, J. C. Maan, W. G. Van der Wiel, G. Rijnders, D. H. A. Blank, and H. Hilgenkamp, "Magnetic effects at the interface between non-magnetic oxides," NATURE MATERIALS, vol. 6, pp. 493-496, 2007.

[9] C. Bell, S. Harashima, Y. Hikita, and H. Y. Hwang, "Thickness dependence of the mobility at the $\mathrm{LaAlO}_{3} / \mathrm{SrTiO}_{3}$ interface," APPLIED PHYSICS LETTERS, vol. 94 , p. $222111,2009$.

[10] N. Reyren, S. Thiel, A. D. Caviglia, L. F. Kourkoutis, G. Hammerl, C. Richter, C. W. Schneider, T. Kopp, A.-S. Ruetschi, D. Jaccard, M. Gabay, D. A. Muller, J.-M. Triscone, and J. Mannhart, "Superconducting interfaces between insulating oxides," SCIENCE, vol. 317, pp. 1196-1199, 2007.

[11] M. Ben Shalom, C. W. Tai, Y. Lereah, M. Sachs, E. Levy, D. Rakhmilevitch, A. Palevski, and Y. Dagan, "Anisotropic magnetotransport at the $\mathrm{SrTiO}_{3} / \mathrm{LaAlO}_{3}$ interface," PHYSICAL REVIEW B, vol. 80, p. 140403, 2009. 
[12] A. D. Caviglia, S. Gariglio, N. Reyren, D. Jaccard, T. Schneider, M. Gabay, S. Thiel, G. Hammerl, J. Mannhart, and J. M. Triscone, "Electric field control of the $\mathrm{LaAlO}_{3} / \mathrm{SrTiO}_{3}$ interface ground state," NATURE, vol. 456, pp. 624-627, 2008 .

[13] C. Bell, S. Harashima, Y. Kozuka, M. Kim, B. G. Kim, Y. Hikita, and H. Y. Hwang, "Dominant Mobility Modulation by the Electric Field Effect at the $\mathrm{LaAlO}_{3} / \mathrm{SrTiO}_{3}$ Interface," PHYSICAL REVIEW LETTERS, vol. 103, p. 226802, 2009 .

[14] C. Cen, S. Thiel, G. Hammerl, C. W. Schneider, K. E. Andersen, C. S. Hellberg, J. Mannhart, and J. Levy, "Nanoscale control of an interfacial metal-insulator transition at room temperature," NATURE MATERIALS, vol. 7, pp. 298-302, 2008 .

[15] C. Cen, S. Thiel, J. Mannhart, and J. Levy, "Oxide Nanoelectronics on Demand," SCIENCE, vol. 323, pp. 1026-1030, 2009.

[16] S. Smadici, P. Abbamonte, A. Bhattacharya, X. Zhai, B. Jiang, A. Rusydi, J. N. Eckstein, S. D. Bader, and J.-M. Zuo, "Electronic reconstruction at $\mathrm{SrMnO}_{3}-\mathrm{LaMnO}_{3}$ superlattice interfaces," PHYSICAL REVIEW LETTERS, vol. 99, p. 196404, 2007.

[17] C. Adamo, X. Ke, P. Schiffer, A. Soukiassian, M. Warusawithana, L. Maritato, and D. G. Schlom, "Electrical and magnetic properties of $\left(\mathrm{SrMnO}_{3}\right)(\mathrm{n}) /\left(\mathrm{LaMnO}_{3}\right)(2 \mathrm{n})$ superlattices," APPLIED PHYSICS LETTERS, vol. 92, p. 112508, 2008.

[18] A. Bhattacharya, S. J. May, S. G. E. t. Velthuis, M. Warusawithana, X. Zhai, B. Jiang, J. M. Zuo, M. R. Fitzsimmons, S. D. Bader, and J. N. Eckstein, "Metal-insulator transition and its relation to magnetic structure in $\left(\mathrm{LaMnO}_{3}\right)(2 \mathrm{n}) /\left(\mathrm{SrMnO}_{3}\right)(\mathrm{n})$ superlattices," PHYSICAL REVIEW LETTERS, vol. 100, p. 257203, 2008.

[19] A. Gozar, G. Logvenov, L. F. Kourkoutis, A. T. Bollinger, L. A. Giannuzzi, D. A. Muller, and I. Bozovic, "High-temperature interface superconductivity between metallic and insulating copper oxides," NATURE, vol. 455, pp. 782-785, 2008.

[20] P. Perna, D. Maccariello, M. Radovic, U. S. di Uccio, I. Pallecchi, M. Codda, D. Marre, C. Cantoni, J. Gazquez, M. Varela, S. J. Pennycook, and F. M. Granozio, "Conducting interfaces between band insulating oxides: The $\mathrm{LaGaO}_{3} / \mathrm{SrTiO}_{3}$ heterostructure," APPLIED PHYSICS LETTERS, vol. 97, p. 152111, 2010.

[21] Y. Hotta, T. Susaki, and H. Y. Hwang, "Polar discontinuity doping of the $\mathrm{LaVO}_{3} / \mathrm{SrTiO}_{3}$ interface," PHYSICAL REVIEW LETTERS, vol. 99, p. 236805, 2007.

[22] A. Kalabukhov, R. Gunnarsson, T. Claeson, and D. Winkler, "Electrical transport properties of polar heterointerface between $\mathrm{KTaO}_{3}$ and $\mathrm{SrTiO}_{3}$," arXiv:0704.1050v1.

[23] K. Shibuya, T. Ohnishi, M. Lippmaa, and M. Oshima, "Metallic conductivity at the $\mathrm{CaHfO}_{3} / \mathrm{SrTiO}_{3}$ interface," APPLIED PHYSICS LETTERS, vol. 91, p. 232106, 2007. 
[24] N. C. Bristowe, E. Artacho, and P. B. Littlewood, "Oxide superlattices with alternating p and n interfaces," PHYSICAL REVIEW B, vol. 80, p. 045425, 2009.

[25] R. Pentcheva, M. Huijben, K. Otte, W. E. Pickett, J. E. Kleibeuker, J. Huijben, H. Boschker, D. Kockmann, W. Siemons, G. Koster, H. J. W. Zandvliet, G. Rijnders, D. H. A. Blank, H. Hilgenkamp, and A. Brinkman, "Parallel Electron-Hole Bilayer Conductivity from Electronic Interface Reconstruction," PHYSICAL REVIEW LETTERS, vol. 104, p. 166804, 2010.

[26] P. R. Willmott, S. A. Pauli, R. Herger, C. M. Schlepuetz, D. Martoccia, B. D. Patterson, B. Delley, R. Clarke, D. Kumah, C. Cionca, and Y. Yacoby, "Structural basis for the conducting interface between $\mathrm{LaAlO}_{3}$ and $\mathrm{SrTiO}_{3}$," PHYSICAL REVIEW LETTERS, vol. 99, p. 155502, 2007.

[27] G. W. J. Hassink, Two-dimensional electron layers in perovskite oxides. $\mathrm{PhD}$ thesis, University of Twente, 2009.

[28] J. Seidel, L. W. Martin, Q. He, Q. Zhan, Y. H. Chu, A. Rother, M. E. Hawkridge, P. Maksymovych, P. Yu, M. Gajek, N. Balke, S. V. Kalinin, S. Gemming, F. Wang, G. Catalan, J. F. Scott, N. A. Spaldin, J. Orenstein, and R. Ramesh, "Conduction at domain walls in oxide multiferroics," NATURE MATERIALS, vol. 8, pp. 229-234, 2009.

[29] M. Huijben, A. Brinkman, G. Koster, G. Rijnders, H. Hilgenkamp, and D. H. A. Blank, "Structure-Property Relation of $\mathrm{SrTiO}_{3} / \mathrm{LaAlO}_{3}$ Interfaces," ADVANCED MATERIALS, vol. 21, pp. 1665-1677, 2009.

[30] H. Chen, A. M. Kolpak, and S. Ismail-Beigi, "Electronic and Magnetic Properties of $\mathrm{SrTiO}_{3} / \mathrm{LaAlO}_{3}$ Interfaces from First Principles," ADVANCED MATERIALS, vol. 22 , pp. 2881-2899, 2010.

[31] R. Pentcheva and W. E. Pickett, "Charge localization or itineracy at $\mathrm{LaAlO}_{3} / \mathrm{SrTiO}_{3}$ interfaces: Hole polarons, oxygen vacancies, and mobile electrons," PHYSICAL REVIEW B, vol. 74, p. 035112, 2006.

[32] S. Gemming and G. Seifert, " $\mathrm{SrTiO}_{3}(001)-\mathrm{LaAlO}_{3}(001)$ multilayers: A density-functional investigation," ACTA MATERIALIA, vol. 54, pp. 4299-4306, 2006.

[33] M. S. Park, S. H. Rhim, and A. J. Freeman, "Charge compensation and mixed valency in $\mathrm{LaAlO}_{3} / \mathrm{SrTiO}_{3}$ heterointerfaces studied by the FLAPW method," PHYSICAL REVIEW B, vol. 74, p. 205416, 2006.

[34] J. M. Albina, M. Mrovec, B. Meyer, and C. Elsaesser, "Structure, stability, and electronic properties of $\mathrm{SrTiO}_{3} / \mathrm{LaAlO}_{3}$ and $\mathrm{SrTiO}_{3} / \mathrm{SrRuO}_{3}$ interfaces," PHYSICAL REVIEW B, vol. 76, p. 165103, 2007.

[35] Z. Zhong and P. J. Kelly, "Electronic-structure-induced reconstruction and magnetic ordering at the $\mathrm{LaAlO}_{3}-\mathrm{SrTiO}_{3}$ interface," EPL, vol. 84, p. 27001, 2008

[36] R. Pentcheva and W. E. Pickett, "Ionic relaxation contribution to the electronic reconstruction at the n-type $\mathrm{LaAlO}_{3} / \mathrm{SrTiO}_{3}$ interface," PHYSICAL REVIEW B, vol. 78 , p. 205106, 2008. 
[37] Z. Popovic, S. Satpathy, and R. Martin, "Origin of the Two-Dimensional Electron Gas Carrier Density at the $\mathrm{LaAlO}_{3}$ on $\mathrm{SrTiO}_{3}$ Interface," PHYSICAL REVIEW LETTERS, vol. 101, p. 256801, 2008.

[38] K. Janicka, J. P. Velev, and E. Y. Tsymbal, "Quantum Nature of Two-Dimensional Electron Gas Confinement at $\mathrm{LaAlO}_{3} / \mathrm{SrTiO}_{3}$ Interfaces," PHYSICAL REVIEW LETTERS, vol. 102, p. 106803, 2009.

[39] J. Lee and A. Demkov, "Charge origin and localization at the n-type $\mathrm{SrTiO}_{3} / \mathrm{LaAlO}_{3}$ interface," PHYSICAL REVIEW B, vol. 78, p. 193104, 2008.

[40] R. Pentcheva and W. E. Pickett, "Avoiding the Polarization Catastrophe in $\mathrm{LaAlO}_{3}$ Overlayers on $\mathrm{SrTiO}_{3}(001)$ through Polar Distortion," PHYSICAL REVIEW LETTERS, vol. 102, p. 107602, 2009.

[41] H. Chen, A. M. Kolpak, and S. Ismail-Beigi, "Fundamental asymmetry in interfacial electronic reconstruction between insulating oxides: An ab initio study," PHYSICAL REVIEW B, vol. 79, p. 161402, 2009.

[42] W. Son, E. Cho, B. Lee, J. Lee, and S. Han, "Density and spatial distribution of charge carriers in the intrinsic n-type $\mathrm{LaAlO}_{3}-\mathrm{SrTiO}_{3}$ interface," PHYSICAL REVIEW B, vol. 79, p. 245411, 2009.

[43] M. Stengel and D. Vanderbilt, "Berry-phase theory of polar discontinuities at oxide-oxide interfaces," PHYSICAL REVIEW B, vol. 80, p. 241103, 2009.

[44] Y. Li, S. N. Phattalung, S. Limpijumnong, and J. Yu, "Oxygen-vacancy-induced charge carrier in n-type interface of $\mathrm{LaAlO}_{3}$ overlayer on $\mathrm{SrTiO}_{3}(001)$ : interface vs bulk doping carrier," arXiv:0912.4805v1.

[45] L. Zhang, X.-F. Zhou, H.-T. Wang, J.-J. Xu, J. Li, E. G. Wang, and S.-H. Wei, "Origin of insulating behavior of the $p$-type $\mathrm{LaAlO}_{3} / \mathrm{SrTiO}_{3}$ interface: Polarization-induced asymmetric distribution of oxygen vacancies," PHYSICAL REVIEW B, vol. 82, p. 125412, 2010.

[46] Z. Zhong, P. X. Xu, and P. J. Kelly, "Polarity-induced oxygen vacancies at $\mathrm{LaAlO}_{3} / \mathrm{SrTiO}_{3}$ interfaces," PHYSICAL REVIEW B, vol. 82, p. 165127, 2010.

[47] M. Basletic, J. L. Maurice, C. Carretero, G. Herranz, O. Copie, M. Bibes, E. Jacquet, K. Bouzehouane, S. Fusil, and A. Barthelemy, "Mapping the spatial distribution of charge carriers in $\mathrm{LaAlO}_{3} / \mathrm{SrTiO}_{3}$ heterostructures," NATURE MATERIALS, vol. 7, pp. 621-625, 2008.

[48] M. Sing, G. Berner, K. Goss, A. Mueller, A. Ruff, A. Wetscherek, S. Thiel, J. Mannhart, S. A. Pauli, C. W. Schneider, P. R. Willmott, M. Gorgoi, F. Schaefers, and R. Claessen, "Profiling the Interface Electron Gas of $\mathrm{LaAlO}_{3} / \mathrm{SrTiO}_{3}$ Heterostructures with Hard X-Ray Photoelectron Spectroscopy," PHYSICAL REVIEW LETTERS, vol. 102, p. 176805, 2009.

[49] T. Fix, F. Schoofs, J. L. MacManus-Driscoll, and M. G. Blamire, "Charge Confinement and Doping at $\mathrm{LaAlO}_{3} / \mathrm{SrTiO}_{3}$ Interfaces," PHYSICAL REVIEW LETTERS, vol. 103, p. 166802, 2009.

[50] N. Reyren, S. Gariglio, A. D. Caviglia, D. Jaccard, T. Schneider, and J. M. Triscone, "Anisotropy of the superconducting transport properties of the $\mathrm{LaAlO}_{3} / \mathrm{SrTiO}_{3}$ interface," APPLIED PHYSICS LETTERS, vol. 94, p. 112506, 2009 . 
[51] J. L. Maurice, C. Carretero, M. J. Casanove, K. Bouzehouane, S. Guyard, E. Larquet, and J. P. Contour, "Electronic conductivity and structural distortion at the interface between insulators $\mathrm{SrTiO}_{3}$ and $\mathrm{LaAlO}_{3}, "$ PHYSICA STATUS SOLIDI A-APPLICATIONS AND MATERIALS SCIENCE, vol. 203, pp. 2209-2214, 2006.

[52] V. Vonk, M. Huijben, K. J. I. Driessen, P. Tinnemans, A. Brinkman, S. Harkema, and $\mathrm{H}$. Graafsma, "Interface structure of $\mathrm{SrTiO}_{3} / \mathrm{LaAlO}_{3}$ at elevated temperatures studied in situ by synchrotron x rays," PHYSICAL REVIEW B, vol. 75, p. $235417,2007$.

[53] C. L. Jia, S. B. Mi, M. Faley, U. Poppe, J. Schubert, and K. Urban, "Oxygen octahedron reconstruction in the $\mathrm{SrTiO}_{3} / \mathrm{LaAlO}_{3}$ heterointerfaces investigated using aberration-corrected ultrahigh-resolution transmission electron microscopy," PHYSICAL REVIEW B, vol. 79, p. 081405, 2009.

[54] M. Salluzzo, J. C. Cezar, N. B. Brookes, V. Bisogni, G. M. De Luca, C. Richter, S. Thiel, J. Mannhart, M. Huijben, A. Brinkman, G. Rijnders, and G. Ghiringhelli, "Orbital Reconstruction and the Two-Dimensional Electron Gas at the $\mathrm{LaAlO}_{3} / \mathrm{SrTiO}_{3}$ Interface," PHYSICAL REVIEW LETTERS, vol. 102, p. 166804, 2009.

[55] K. Yoshimatsu, R. Yasuhara, H. Kumigashira, and M. Oshima, "Origin of metallic states at the heterointerface between the band insulators $\mathrm{LaAlO}_{3}$ and $\mathrm{SrTiO}_{3}$," PHYSICAL REVIEW LETTERS, vol. 101, p. 026802, 2008.

[56] Y. Segal, J. H. Ngai, J. W. Reiner, F. J. Walker, and C. H. Ahn, "X-ray photoemission studies of the metal-insulator transition in $\mathrm{LaAlO}_{3} / \mathrm{SrTiO}_{3}$ structures grown by molecular beam epitaxy," PHYSICAL REVIEW B, vol. 80, p. $241107,2009$.

[57] A. S. Kalabukhov, Y. A. Boikov, I. T. Serenkov, V. I. Sakharov, V. N. Popok, R. Gunnarsson, J. Borjesson, N. Ljustina, E. Olsson, D. Winkler, and T. Claeson, "Cationic Disorder and Phase Segregation in $\mathrm{LaAlO}_{3} / \mathrm{SrTiO}_{3}$ Heterointerfaces Evidenced by Medium-Energy Ion Spectroscopy," PHYSICAL REVIEW LETTERS, vol. 103, p. 146101, 2009.

[58] S. Chambers, M. Engelhard, V. Shutthanandan, Z. Zhu, T. Droubay, L. Qiao, P. Sushko, L. H. Feng, T, T. Gustafsson, E. Garfunkel, A. Shah, J.-M. Zuo, and Q. Ramasse, "Instability, Intermixing and Electronic Structure at the Epitaxial $\mathrm{LaAlO}_{3} / \mathrm{SrTiO}_{3}$ (001) Heterojunction ," arXiv:1006.1378.

[59] G. Koster, B. Kropman, G. Rijnders, D. Blank, and H. Rogalla, "Quasi-ideal strontium titanate crystal surfaces through formation of strontium hydroxide," APPLIED PHYSICS LETTERS, vol. 73, pp. 2920-2922, 1998.

[60] NIST Standard Reference Database 71, NIST Electron Inelastic-Mean-Free-Path Database: Ver. 1.1, URL http://www.nist.gov/srd/nist71.htm.

[61] S. Ishibashi and K. Terakura, "Analysis of Screening Mechanisms for Polar Discontinuity for $\mathrm{LaAlO}_{3} / \mathrm{SrTiO}_{3}$ Thin Films Based on Ab initio Calculations," JOURNAL OF THE PHYSICAL SOCIETY OF JAPAN, vol. 77, p. 104706, 2008.

[62] W. Son and S. Han. private communication, 2009. 
[63] V. Leca, Heteroepitaxial growth of copper oxide superconductors by Pulsed Laser Deposition. PhD thesis, University of Twente, 2003.

[64] G. Rijnders, D. Blank, J. Choi, and C. Eom, "Enhanced surface diffusion through termination conversion during epitaxial $\mathrm{SrRuO}_{3}$ growth," APPLIED PHYSICS LETTERS, vol. 84, pp. 505-507, 2004.

[65] L. van der Pauw, "A method of measuring the resistivity and Hall coefficient on lamellae of arbitrary shape," Philips Technical Review, vol. 20, pp. 220-224, 1958.

[66] J. L. Maurice, G. Herranz, C. Colliex, I. Devos, C. Carretero, A. Barthelemy, K. Bouzehouane, S. Fusil, D. Imhoff, E. Jacquet, F. Jomard, D. Ballutaud, and M. Basletic, "Electron energy loss spectroscopy determination of Ti oxidation state at the (001) $\mathrm{LaAlO}_{3} / \mathrm{SrTiO}_{3}$ interface as a function of $\mathrm{LaAlO}_{3}$ growth conditions," EPL, vol. 82, p. 17003, 2008.

[67] K. A. Müller and H. Burkard, "SrTiO ${ }_{3}$ : An intrinsic quantum paraelectric below 4 K," Phys. Rev. B, vol. 19, pp. 3593-3602, 1979.

[68] A. Savoia, D. Paparo, P. Perna, Z. Ristic, M. Salluzzo, F. M. Granozio, U. S. di Uccio, C. Richter, S. Thiel, J. Mannhart, and L. Marrucci, "Polar catastrophe and electronic reconstructions at the $\mathrm{LaAlO}_{3} / \mathrm{SrTiO}_{3}$ interface: Evidence from optical second harmonic generation," PHYSICAL REVIEW B, vol. 80, p. 075110, 2009 .

[69] N. Ogawa, K. Miyano, M. Hosoda, T. Higuchi, C. Bell, Y. Hikita, and H. Y. Hwang, "Enhanced lattice polarization in $\mathrm{SrTiO}_{3} / \mathrm{LaAlO}_{3}$ superlattices measured using optical second-harmonic generation," PHYSICAL REVIEW B, vol. 80, p. 081106, 2009.

[70] H. Li, W. Si, R. Wang, Y. Xuan, B. Liu, and X. Xi, "Dielectric properties of $\mathrm{SrTiO}_{3}$ thin films grown on various perovskite electrodes by pulsed laser deposition," MATERIALS SCIENCE AND ENGINEERING B-SOLID STATE MATERIALS FOR ADVANCED TECHNOLOGY, vol. 56, pp. 218-222, 1998.

[71] J. He, A. Borisevich, S. V. Kalinin, S. J. Pennycook, and S. T. Pantelides, "Control of Octahedral Tilts and Magnetic Properties of Perovskite Oxide Heterostructures by Substrate Symmetry," PHYSICAL REVIEW LETTERS, vol. 105, p. 227203, 2010.

[72] Y. Yao and H. Fu, "Influence of rotational instability on the polarization structure of $\mathrm{SrTiO}_{3}$," arXiv:1009.4689v1.

[73] T. Okuda, K. Nakanishi, S. Miyasaka, and Y. Tokura, "Large thermoelectric response of metallic perovskites: $\mathrm{Sr}_{1-x} \mathrm{La}_{x} \mathrm{TiO}_{3}(0 \leq \mathrm{x} \leq 0.1)$," PHYSICAL REVIEW B, vol. 63, p. 113104, 2001. 


\title{
Chapter 3
}

\section{Fabrication and properties of $\mathbf{L a}_{0.67} \mathbf{S r}_{0.33} \mathrm{MnO}_{3}$ thin films}

\begin{abstract}
In this chapter, an overview is given of the fabrication and properties of the $\mathrm{La}_{0.67} \mathrm{Sr}_{0.33} \mathrm{MnO}_{3}$ (LSMO) thin films used in this thesis. The films were grown by pulsed laser deposition, monitored with in situ reflection high energy electron diffraction. The initial film growth occurred in a predominantly layer-by-layer fashion which changed to the steady state growth mode during deposition, resulting in a smooth surface with islands with unit cell step height. Structural characterization showed that the LSMO films were grown in a fully coherent fashion with respect to the underlying substrates. The characterization of the magnetic and electric transport properties of the films showed a saturation magnetization of $4.0 \mu_{\mathrm{B}} / \mathrm{Mn}$, a Curie temperature of $350 \mathrm{~K}$ and a residual resistivity of $70 \mu \Omega \mathrm{cm}$. X-ray photoelectron spectroscopy measurements showed an absence of chemical impurities and no evidence for surface segregation. Scanning transmission electron microscopy showed that multilayer structures with sharp interfaces were realized. All results indicate that high quality films, comparable to the best examples found in literature, were fabricated. The ability to grow high quality films and multilayer structures is an important prerequisite for the research in the later chapters in this thesis.
\end{abstract}

\subsection{Introduction}

The discovery of colossal magnetoresistance in the perovskite manganites in 1993 resulted in an enormous amount of research [1, 2]. $\mathrm{La}_{1-x} \mathrm{Sr}_{x} \mathrm{MnO}_{3}$ (LSMO) has small magnetoresistance compared to other manganites such as $\mathrm{La}_{1-x} \mathrm{Ca}_{x} \mathrm{MnO}_{3}$, 
due to its large bandwidth. It is, however, the only simple perovskite manganite with a Curie temperature larger than room temperature. For practical applications, as well as fundamental studies, manganite thin films are of great importance and several deposition techniques were explored to realize manganite thin films. As the manganites have a complicated phase diagram, control of the ratios between the cations and the oxygen stoichiometry is crucial. Several early reports on the growth of LSMO thin films can be found. However, many films were optimized for the largest amount of magnetoresistance which does not necessarily result in high quality epitaxy, due to the large magnetoresistance at grain boundaries $[3,4]$. The other important property of LSMO, $100 \%$ spin polarization, requires electronic homogeneity, and therefore high quality epitaxy, for use in devices such as magnetic tunnel junctions. As these devices have a multilayer structure, next to the electronic properties, also the surface morphology is important as the surface is the starting point for the growth of the next layer. Several groups have optimized LSMO growth for multilayer devices, using either pulsed laser deposition (PLD) or molecular beam epitaxy [5-8].

Here, PLD was used, as stoichiometric transfer of material from the target to the sample is relatively easy to achieve with this technique and the kinetic energy of the arriving species can be tuned in a wide range to control the growth process. Furthermore, PLD is compatible with a high oxygen background pressure which avoids oxygen non-stoichiometry in the films.

The aim of this chapter is to present the properties of the LSMO thin films and to demonstrate that high quality film growth was achieved. This is a vital step for the research in the subsequent chapters in this thesis as no conclusions can be drawn about intrinsic properties of the material if the films are not of the highest quality. So, the growth of the films is described together with an analysis of the surface morphology with reflection high energy electron diffraction (RHEED) and scanning probe microscopy (SPM). X-ray diffraction (XRD) measurements showed that the samples are single crystalline and coherently strained to the substrate surface lattice parameters. The electronic structure was investigated with x-ray photoemission spectroscopy (XPS) and the interface atomic structure was measured with scanning transmission electron microscopy (STEM). The films showed functional properties, magnetization and conductivity, of similar quality to those of the bulk single crystal references. In the final section, the properties of the LSMO thin films are compared with films described in literature.

\section{$3.2 \quad \mathrm{La}_{1-\mathrm{x}} \mathrm{Sr}_{\mathrm{x}} \mathrm{MnO}_{3}$}

$\mathrm{La}_{1-\mathrm{x}} \mathrm{Sr}_{\mathrm{x}} \mathrm{MnO}_{3}$ has a very rich phase diagram due to the charge, orbital, spin and lattice degrees of freedom in the system. In this section a short overview is given of the main magnetic interactions and features in the phase diagram. A more detailed description of the system can be found elsewhere [9]. First the relevant $\mathrm{Mn}^{3+}$ and $\mathrm{Mn}^{4+}$ ions and their magnetic interactions are described, next the phase diagram is discussed. 


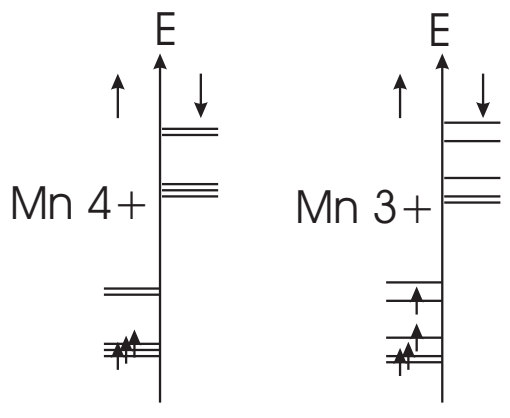

Figure 3.1: A sketch of the energy of the different d orbitals on $\mathrm{Mn}^{4+}$ (left) and $\mathrm{Mn}^{3+}$ (right) ions. In order to illustrate the number of orbitals with

a certain energy degenerate orbitals are slightly separated in energy.

\subsubsection{Magnetic interactions between manganese ions}

Manganese is a transition metal with $\mathrm{d} 7$ configuration. In ionic form $\mathrm{Mn}^{2+}, \mathrm{Mn}^{3+}$ and $\mathrm{Mn}^{4+}$ exist and for the $\mathrm{La}_{1-\mathrm{x}} \mathrm{Sr}_{\mathrm{x}} \mathrm{MnO}_{3}$ phase diagram the latter two configurations are present. $\mathrm{Mn}^{3+}\left(\mathrm{Mn}^{4+}\right)$ has 4 (3) electrons in the d shell. Due to the strong octahedral crystal field in the perovskite structure the orbitals are split in low energy $t_{2 g}$ and high energy $e_{g}$ orbitals. Strong Hund's rule coupling results in an energy splitting between spin up and spin down states. This splitting is larger than the crystal field splitting, so the $\mathrm{Mn}^{3+}$ ions have a spin of $\frac{4}{2}$ and $\mathrm{Mn}^{4+}$ ions have a spin of $\frac{3}{2}$. This is illustrated in figure 3.1. The $\mathrm{Mn}^{3+}$ ion is susceptible to a Jahn Teller distortion which deforms the oxygen octahedron. The octahedron is elongated in one direction ( $\mathrm{z}$ in this case) and contracted in the other two. This splits the previously degenerate $t_{2 g}$ levels into low energy $d_{x z}$ and $d_{y z}$ orbitals and a higher energy $d_{x y}$ orbital. The $e_{g}$ orbitals are split as well, the low energy $d_{3 z^{2}-r^{2}}$ orbital is occupied.

Between nearest neighbour Mn ions, several magnetic interactions exist. Non itinerant electrons can virtually hop to a neighbouring site to gain kinetic energy. This process, called superexchange interaction, is only possible if the orbital to which the virtual hopping occurs is empty and therefore depends on the relative spin configuration. It can be understood with the Goodenough Kanamori rules $[10,11]$ which state that the magnetic superexchange interaction between half filled orbitals is antiferromagnetic and that the interaction between a half filled orbital and an empty or fully filled orbital is ferromagnetic. Next to superexchange also double exchange is important for the manganites. Opposed to the virtual electron transfer in superexchange, double exchange describes real electron transfer between orbitals. For example the $\mathrm{e}_{\mathrm{g}}$ electron on a $\mathrm{Mn}^{3+}$ ion can hop to a $\mathrm{Mn}^{4+}$ ion, this gains kinetic energy for the $e_{g}$ electron. As the hopping depends on the spin alignment of the two ions, the double exchange mechanism favours ferromagnetism [12-14]. Next to these magnetic interactions also the charge, orbital and lattice degrees of freedom play a role in determining the properties of manganite materials. 


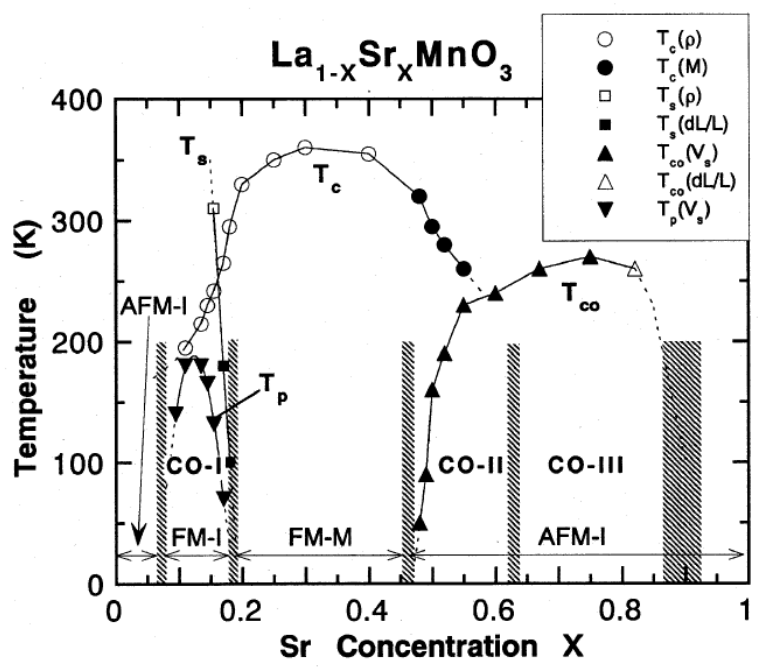

Figure 3.2: The phase diagram of LSMO as taken from reference [15]. $T_{\mathrm{C}}$ shows the Curie temperature obtained from either resistivity $(\rho(T)$, open circles) or magnetization $\left(M(T)\right.$, solid circles) measurements and $T_{\mathrm{s}}$ shows the structural transition temperature as determined from resistivity (open square) or dilatation $\left(\mathrm{d} L(T) / L\right.$, solid square) measurements. $T_{\text {co }}$ shows the charge-ordering temperature as determined from sound velocity $\left(V_{\mathrm{s}}(T)\right.$, solid triangle) or dilatation (open triangle) measurements and $T_{\mathrm{p}}$ shows the polaron-ordering temperature obtained from sound velocity (solid inverted triangle) measurements. The shaded regions correspond to the borders of the three types of charge orderings (CO-I, CO-II and CO-III). AFM-I, FM-I and FM-M imply antiferromagnetic insulator, ferromagnetic insulator and ferromagnetic metal, respectively. This figure has been reproduced with permission from the author. (c) 1998 the Physical Society of Japan.

\subsubsection{The phase diagram}

The phase diagram of $\mathrm{La}_{1-\mathrm{x}} \mathrm{Sr}_{\mathrm{x}} \mathrm{MnO}_{3}$ is shown in figure $3.2[15,16]$. On the left side is the parent compound $\mathrm{LaMnO}_{3}$ (LMO) in which all manganese ions have the $\mathrm{Mn}^{3+}$ valence state. On the right side is the $\mathrm{SrMnO}_{3}$ (SMO) compound with $\mathrm{Mn}^{4+}$ valence. Several structural phases are present. The most important feature is the orthorhombic/rhombohedral transition denoted with $T_{\mathrm{s}}$ which crosses the 0 $\mathrm{K}$ axis at a doping of $x=0.2$. With the substitution of La by the bigger ion $\mathrm{Sr}$ and the simultaneous substitution of smaller $\mathrm{Mn}^{4+}$ ions for $\mathrm{Mn}^{3+}$ ions the tolerance factor increases and this causes the orthorhombic to rhombohedral transition. The dome of the ferromagnetic metallic region of the phase diagram can be seen around $x=\frac{1}{3}$ doping. Next the various magnetic phases are discussed in more detail.

The parent compound LMO is a Mott insulator. Its ground state is characterized by an orbital ordering pattern and A-type antiferromagnetic order. In the ab plane nearest neighbour $\mathrm{Mn}$ ions have alternating $\mathrm{d}_{3 \mathrm{x}^{2}-\mathrm{r}^{2}}$ and $\mathrm{d}_{3 \mathrm{y}^{2}-\mathrm{r}^{2}}$ orbitals, along the 


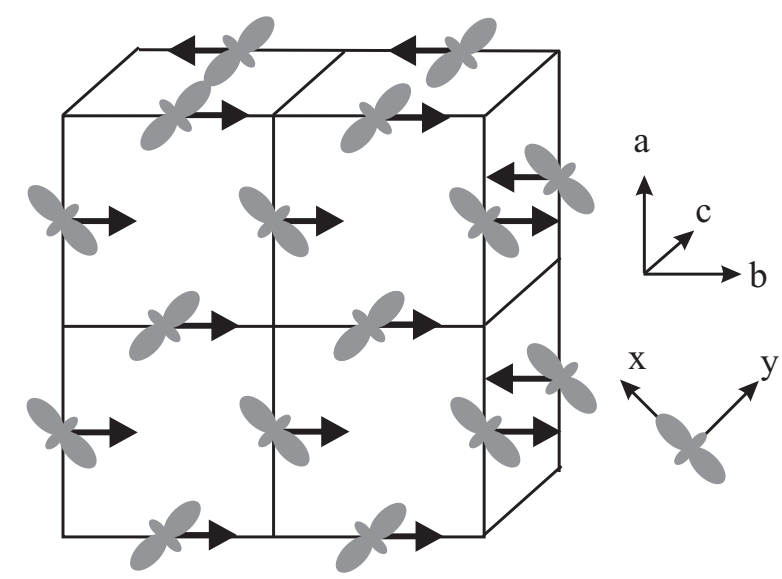

Figure 3.3: A schematic of the orbital ordering and the A-type antiferromagnetic ordering in LMO.

c axis the orbital configuration is constant [17]. The orbital order is stabilized by the Jahn Teller distortion. The orbital ordering transition temperature is $750 \mathrm{~K}$, which corresponds to a large energy scale in comparison to the other features in the phase diagram. LMO's Néel temperature is $120 \mathrm{~K}$, below which the spins align ferromagnetically in the ab plane and antiferromagnetically along the $\mathbf{c}$ axis, in accordance with the Goodenough Kanamori rules. The magnetization is aligned with the $\mathbf{b}$ axis, approximately 45 degrees with respect to $x$ and $y$. A schematic of the orbital and spin configuration in LMO is given in figure 3.3.

With the doping of holes in LMO, the antiferromagnetic coupling along the $\mathbf{c}$ axis is reduced, resulting in a canted antiferromagnetic state. With progressive doping, this results in a ferromagnetic insulating state for doping levels of $0.1<x<0.18$ and in the ferromagnetic metallic state at doping $0.18<x<0.45$. The ferromagnetic insulating state can be understood as a state where locally the double exchange mechanism results in ferromagnetic coupling, but globally no charge transport is possible due to the trapping of the carriers by polaron (a bound state of a charge carrier and a lattice distortion) formation. In the ferromagnetic metallic phase, the increase in doping reduces carrier trapping. At optimum doping, $x=\frac{1}{3}$, the Curie temperature of $370 \mathrm{~K}$. At the Curie temperature, the ferromagnet/paramagnet and the metal insulator transition occur simultaneously.

On the other side of the phase diagram the band insulator SMO can be found. Due to the absence of $\mathrm{e}_{\mathrm{g}}$ electrons, no Jahn Teller distortion is present and all exchange interactions are antiferromagnetic. This results in a G-type antiferromagnetic ground state.

Between SMO and LSMO various charge ordered phases exists. These phases typically have a complex orbital and ferromagnetic order as well. For example in the compound at $x=0.5$ doping, ferromagnetic zigzag chains exist together with a checkerboard charge ordered pattern. 
Next to $\mathrm{La}_{1-\mathrm{x}} \mathrm{Sr}_{\mathrm{x}} \mathrm{MnO}_{3}$, perovskite manganites exist with a large variety of $A$-site cations. For example Sr can be changed with $\mathrm{Ca}$ or Ba and La can be exchanged by $\mathrm{Pr}, \mathrm{Nd}, \mathrm{Y}$ and $\mathrm{Sm}$ [18-20]. $\mathrm{La}_{1-\mathrm{x}} \mathrm{Ca}_{\mathrm{x}} \mathrm{MnO}_{3}$ has a similar phase diagram as $\mathrm{La}_{1-\mathrm{x}} \mathrm{Sr}_{\mathrm{x}} \mathrm{MnO}_{3}$, but the Curie temperature of the ferromagnetic metallic phase is reduced. As $\mathrm{Ca}$ has a smaller ionic radius than $\mathrm{Sr}$, the perovskite tolerance factor is reduced. This results in larger oxygen octahedra rotations, which reduces the bandwidth. For a large set of compounds in which La has been replaced, the ferromagnetic metallic phase is completely absent.

\subsection{Thin film growth}

In this section, the growth of the LSMO thin films is described. First, the treatment of the substrates used as template for the growth is discussed. Then, the growth of the thin films by pulsed laser deposition is described. In situ RHEED measurements are presented from which it is concluded that the initial growth occurs in the two dimensional layer-by-layer growth mode, and that a transition to a steady state growth mode occurs for thicker films. Finally, the surface morphology was characterized with SPM, which showed smooth surfaces.

\subsubsection{Substrates}

Several substrates are commercially available for the growth of oxide thin films. A number of substrates which have comparable lattice parameters to LSMO can be used as a template for the growth of the LSMO thin films. They include $\mathrm{NdGaO}_{3}$ (NGO), $\left(\mathrm{LaAlO}_{3}\right)_{0,3}-\left(\mathrm{Sr}_{2} \mathrm{AlTaO}_{6}\right)_{0,7}$ (LSAT), $\mathrm{SrTiO}_{3}(\mathrm{STO})$ and $\mathrm{DyScO}_{3}$ (DSO). The pseudocubic lattice parameters are respectively $3.85,3.87,3.905$ and $3.95 \AA$. STO and LSAT are cubic crystals while NGO and DSO have an orthorhombic crystal structure. In the orthorhombic crystal structure two possibilities exist for a surface with pseudocubic (001) orientation, namely $(001)_{\mathrm{o}}$ and $(110)_{\mathrm{o}}$. (The subscripts o, c and pc denote the orthorhombic, cubic and pseudocubic crystal structure, respectively.) The (110)o surface unit cell has 90 degrees angles with a small difference in the pseudocubic surface unit cell lattice parameters while the (001) o surface unit cell has equal lattice parameters but a small deviation from 90 degrees in the angle between the lattice parameters. For the studies in this thesis, both STO $(001)_{c}$, NGO (110)o and NGO (001)o substrates were used to grow $(001)_{\mathrm{pc}}$ oriented films. Pseudocubic (110) oriented films have been grown as well, using STO $(110)_{\mathrm{c}}$ and NGO (100), NGO (010), and NGO (112)o substrates.

All substrates required some preparation before high quality growth could be achieved. All substrates were first ultrasonically cleaned with acetone and ethanol. The $(110)_{\mathrm{pc}}$ substrates consist of layers of $A B \mathrm{O}$ and $\mathrm{O}_{2}$ planes. The substrates were annealed at $950^{\circ} \mathrm{C}$ in a 1 bar oxygen atmosphere for 1 hour, after which atomically smooth terraces with straight stepedges and half unit cell step height $(2.7 \AA)$ could be observed. This indicates a single surface termination but it is no clear whether this is $A B O$ or $\mathrm{O}_{2}$. 
Singly $\mathrm{TiO}_{2}$ terminated STO $(001)_{c}$ substrates were obtained with the procedure developed by Koster [21]. In some cases, the annealing step of 2 hours at $950^{\circ} \mathrm{C}$ resulted in some $\mathrm{Sr}$ segregation from the bulk towards the surface $[22,23]$. This could be observed with atomic force microscopy (AFM), either in the phase contrast or with the presence of straight stepedges aligned with the principal crystal directions [24]. Those substrates were treated with $\mathrm{H}_{2} \mathrm{O}$ and the $\mathrm{HF}$ solution again, followed by a 30 minute anneal at $920^{\circ} \mathrm{C}$, after which no $\mathrm{SrO}$ termination could be detected. The treatment of the NGO (110), and NGO (001)o substrates followed a similar two step procedure with a small modification to the $\mathrm{HF}$ solution as described in detail in reference [25].

\subsubsection{Pulsed laser deposition}

The LSMO thin films were grown by PLD (TSST system). The substrate temperature during growth was $750-800{ }^{\circ} \mathrm{C}$ in an oxygen environment of 0.27 mbar. The laser beam was produced by a $248-\mathrm{nm}$-wavelength $\mathrm{KrF}$ excimer laser (LPXPro $^{\mathrm{TM}}$ from Coherent, Inc.) with a typical pulse duration of 20-30 ns. With a 4 by 15 $\mathrm{mm}$ rectangular mask the most homogeneous part of the laser beam was selected. An image of the mask was created on the stoichiometric target (Praxair electronics) with a lens, resulting in a spotsize of $2.3 \mathrm{~mm}^{2}(0.9$ by $2.5 \mathrm{~mm})$. The beam energy was controlled with a variable attenuator or with the laser voltage, yielding a fluence at the target of $2 \mathrm{~J} / \mathrm{cm}^{2}$. The repetition rate was $1 \mathrm{~Hz}$ and the substrate was placed at $5 \mathrm{~cm}$ distance directly opposite to the target. Before deposition, the target was pre-ablated for 2 minutes at $5 \mathrm{~Hz}$ to remove any possible surface contamination. After deposition, the PLD chamber was flooded with pure oxygen (typically 100 mbar) and the samples were cooled down by switching of the heater power. Typically, the cooldown required 2 hours. The optimization of the settings for the growth of LSMO is described in reference [26]. Thicker (>10 nm) films were grown with a repetition rate of $5 \mathrm{~Hz}$ and an oxygen pressure of 0.16 mbar.

\subsubsection{Growth mode}

The growth of the films was studied in situ with RHEED during the growth. The substrate RHEED pattern is shown in figure 3.4 on the top left. The main specular spot is very intense compared to the two side spots. This is the typical signature of $\mathrm{TiO}_{2}$ terminated STO [24]. Kikuchi lines are visible as well, indicating the smoothness of the substrate. The side spots are doubled, which is due to the additional periodicity at the surface from the regularly spaced terrace steps. Figure 3.4, top right, shows the RHEED image of the LSMO film after the deposition of 5 unit cell layers. Clear two dimensional spots are visible, but also 2D streaks are present. The latter are attributed to the scattering of the RHEED beam off the unit cell high steps at the surface. Similar RHEED images were observed after the completion of films with thicknesses up to $40 \mathrm{~nm}$.

The main graph in figure 3.4 shows the intensity of the specular reflection as measured during the initial growth of LSMO. The intensity shows oscillations 


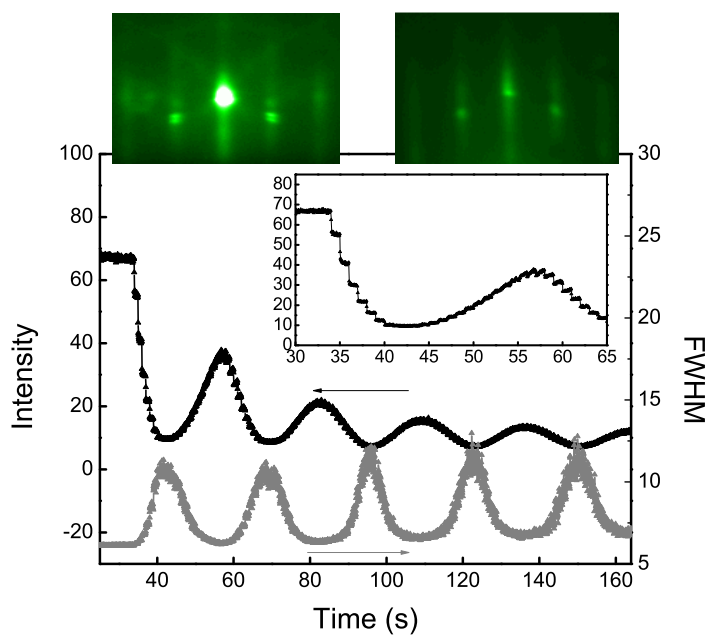

Figure 3.4: RHEED specular spot intensity and FWHM as monitored during the initial growth of LSMO on STO $(001)_{c}$. The inset shows an expanded view of the growth of the first unit cell layer. Two RHEED images are presented, the left image was taken before deposition (at low pressure) and the right image was taken after deposition of 5 unit cell layers.

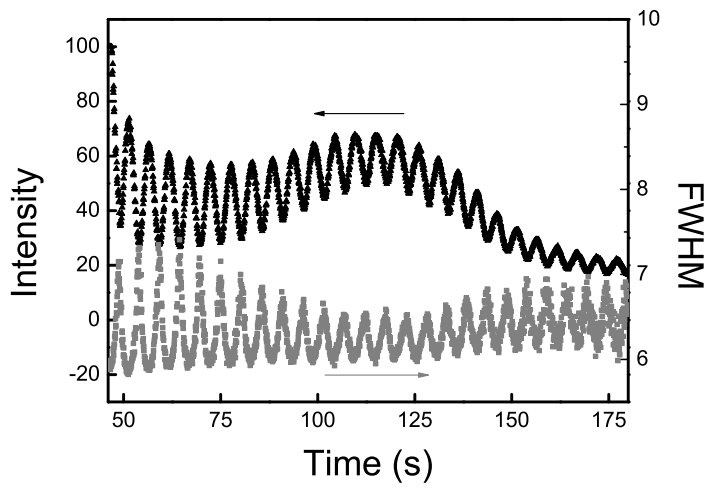

Figure 3.5: RHEED specular spot intensity and FWHM as monitored during the growth of a relatively thick film of LSMO on STO $(001)_{c}$. The graph shows the $7^{\text {th }}$ to $32^{\text {nd }}$ oscillation. A recovery of the RHEED oscillation intensity maximum, followed by a rapid decrease, is observed around the $20^{\text {th }}$ unit cell layer. 
which correspond to the growth of the individual unit cell layers. Within the oscillations, recovery of the intensity after the sudden decrease during the laser burst is observed, as shown more clearly in the inset of the graph. The oscillation amplitude decreases with the amount of material deposited during the first part of the growth. This decrease of the intensity is due to the difference in reflectivity of the STO surface and the LSMO surface, due to the increased scattering from the heavy La ions in the lattice. Finally, the full width at the half maximum (FWHM) of the intensity of the specular spot, measured along the $(10)_{\mathrm{pc}}$ direction, is presented as well. During the growth, the RHEED spots are periodically more streaky, because more stepedge scattering is present at half unit cell layer coverage compared to full unit cell layer coverage. Therefore, the FWHM oscillates as well. The FWHM depends only on the shape of the intensity distribution and not on the total intensity and it is therefore a better indicator of the surface morphology than the main intensity of the reflection. As can be seen in the figure, the FWHM during the growth is almost equal to the FWHM of the substrate reflection indicating a smooth surface morphology. From these measurements it is concluded that the initial stage of the LSMO growth proceeds in the ideal two dimensional layer-bylayer growth mode.

During the growth of the film, an increase of the RHEED oscillation maximum intensity was observed, as presented in figure 3.5. The oscillation intensity maximum typically peaked around 20 to 25 unit cell layers. The origin of the maximum in intensity is not understood. The maximum in intensity was not observed during growth on an $A$-site terminated substrate surface, so it might indicate a termination conversion. After this peak the oscillation intensity maximum decreased rapidly and the oscillation amplitude became comparable to the intensity variations in the laser pulse recovery cycles. It is concluded that the LSMO growth mode during the latter part of the growth is close to the steady state growth mode which is characterized by a relatively constant step density, which is large compared to the step density of the initial substrate surface.

\subsubsection{Surface morphology}

The surface morphology of the films was studied with atomic force microscopy (AFM). The images showed smooth surfaces as shown in the examples presented in figure 3.6. The step and terrace structure of the substrate is still observed on the surface of the films. Figure 3.6a shows the surface morphology of a 20 unit cell layer film which was completely grown in the 2D layer-by-layer growth mode, while figure 3.6b shows the surface morphology of a 30 unit cell layer film where the growth changed to the steady state growth mode. For the latter film islands with unit cell height are observed on the terraces. For LSMO, it is known that off-stoichiometric deposition results in precipitate formation on the surface [27]. Such precipitates have not been observed. 

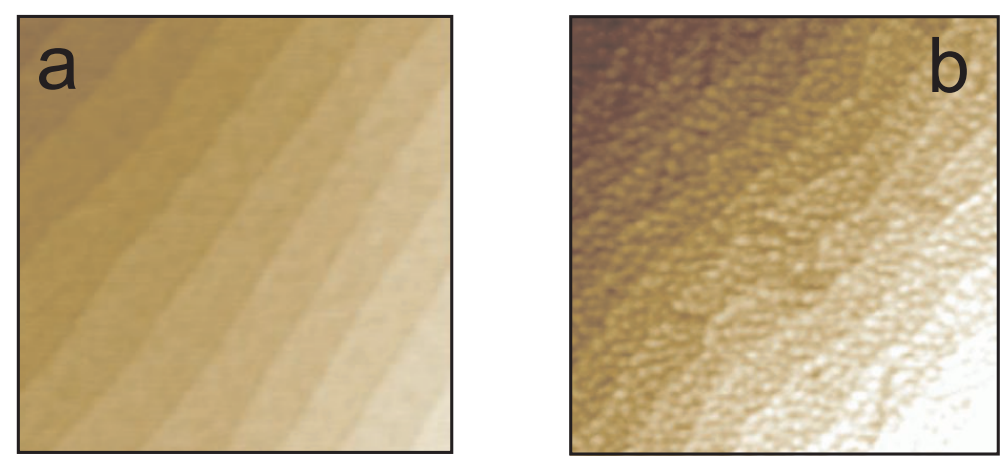

Figure 3.6: Surface morphology as measured with ex situ AFM. a) 20 unit cell layers of LSMO grown on STO. b) 30 unit cell layers of LSMO grown on STO. Both images are 2 by $2 \mu \mathrm{m}$.

\subsection{Crystal structure}

The crystal structure of the LSMO thin films was analyzed with the use of $\mathrm{x}$ ray diffraction (XRD) measurements. The measurements were performed either in Twente using a Bruker D8 Discoverer or at the Stanford Nanocharacterization Laboratory using a PANalytical X'Pert materials diffractometer or at the beamline 7-2 of the Stanford Synchrotron Radiation Laboratory.

The LSMO bulk rhombohedral crystal structure [19] is incommensurate with the square/rectangular surface unit cells of the substrates. The LSMO is therefore under both normal strain as well as shear strain [28]. To accommodate this strain LSMO adopts a different crystal symmetry, namely a distorted (monoclinic) orthorhombic unit cell with space group $\mathrm{P} 2_{1} / \mathrm{m}$ [29]. The orthorhombic unit cell is $(110)_{\mathrm{o}}$ oriented out-of-plane with $[1 \overline{1} 0]_{\mathrm{o}}$ and $[001]_{\mathrm{o}}$ in-plane orientations. To confirm that the LSMO layers were grown in a fully coherent fashion with respect to the underlying substrate, reciprocal lattice maps (RLM) were taken around symmetrical and asymmetrical reflections.

As an example, RLMs around the $(260)_{\mathrm{o}},(444)_{\mathrm{o}},(620)_{\mathrm{o}}$ and $(44 \overline{4})_{\mathrm{o}}$ reflections of a $40 \mathrm{~nm}$ LSMO film grown on STO are shown in figure 3.7. The equal in-plane momentum transfer of the film and substrate peaks indicates a fully coherent film. The very small difference in the $(260)_{\mathrm{o}}$ and $(620)_{\mathrm{o}}$ atomic plane spacings represents a small difference in the $a_{o}$ and $b_{o}$ film lattice parameters. The refined lattice parameters are as follows: $\mathbf{a}_{\mathrm{o}}=5.480 \AA, \mathbf{b}_{\mathrm{o}}=5.483 \AA, \mathbf{c}_{\mathrm{o}}=7.809 \AA, \alpha_{\mathrm{o}}=\beta_{\mathrm{o}}=90^{\circ}$ and $\gamma_{\mathrm{o}}=90.87^{\circ}$. The pseudocubic lattice parameters are $\mathbf{a}_{\mathrm{pc}}=\mathbf{b}_{\mathrm{pc}}=3.905 \AA$ and $\mathbf{c}_{\mathrm{pc}}=3.846 \AA$. The errors in the lattice parameters are $0.001 \AA$ and $0.01^{\circ}$.

Figure 3.8 shows an HK scan (along the out-of-plane direction) of the $(220)_{\mathrm{o}}$ LSMO and $(002)_{\mathrm{c}}$ STO Bragg peaks. Next to the peak, finite thickness fringes are observed whose period corresponds well to the $40 \mathrm{~nm}$ film thickness as estimated from counting the RHEED oscillations. The clear oscillations indicate a high quality sample with a smooth substrate film interface and a smooth film surface, as 


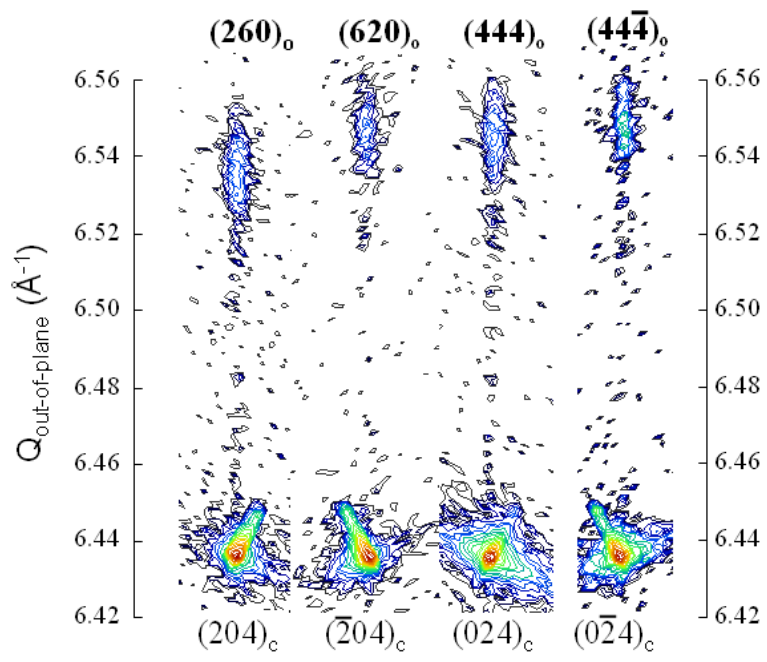

Figure 3.7: Room temperature XRD RLMs around the $(260)_{\mathrm{o}}$, $(444)_{\mathrm{o}}$, $(620)_{0}$ and $(44 \overline{4})_{\text {o }}$ reflections of a $40 \mathrm{~nm}$ LSMO film grown on STO. This measurement was performed at the Stanford Nanocharacterization Laboratory.

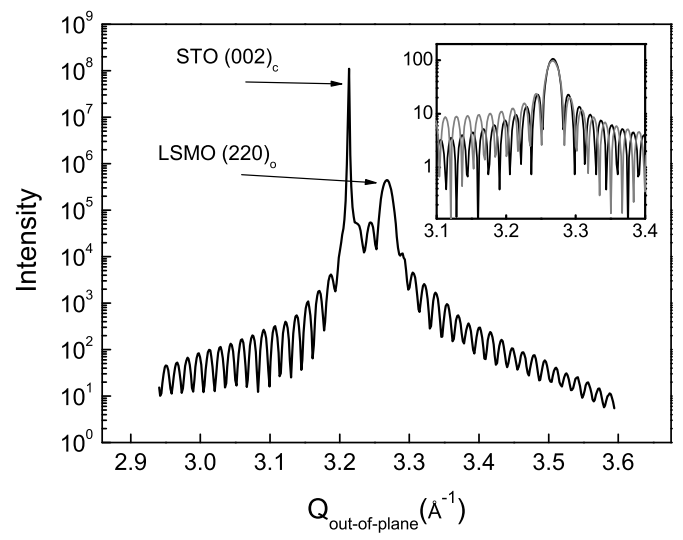

Figure 3.8: Room temperature XRD HK scan (along the out-of-plane direction) of a $40 \mathrm{~nm}$ LSMO/STO sample. The substrate and film Bragg peaks have been indicated and thickness fringes are observed. This measurement was performed at beamline 7-2 of the Stanford Synchrotron Radiation Laboratory. The inset shows a simulated fringe pattern of a $40 \mathrm{~nm}$ LSMO layer (black) and a simulated pattern of a similar layer with unit cell elongation at the interfaces. For the latter simulation 3 unit cells at each interface were elongated by $10 \%$. The elongation results in higher intensity of the fringes for low momentum transfer (Q), as observed in the experimental data. 

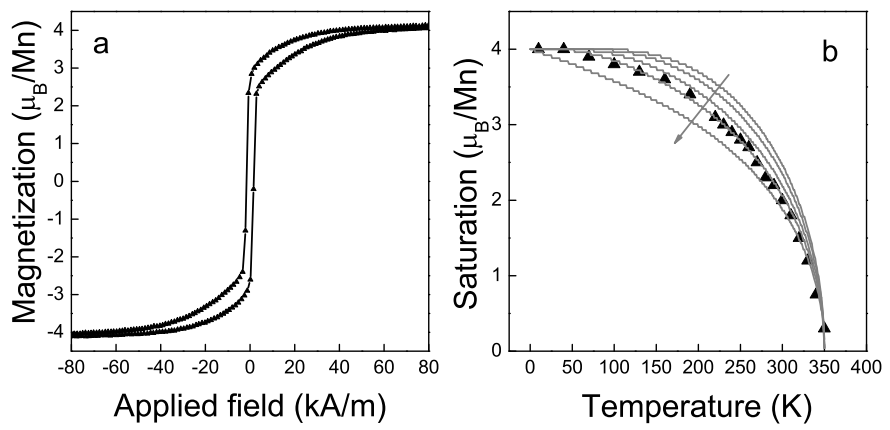

Figure 3.9: Magnetization measurements of $11.5 \mathrm{~nm}$ LSMO grown on STO $(001)_{c}$. a) Hysteresis loop obtained at $10 \mathrm{~K}$ with the field applied in the plane of the film parallel to the $[100]_{\mathrm{pc}}$ LSMO lattice direction. b) Temperature dependence of the saturation magnetization. Datapoints are indicated with black triangles and the curves are calculated from the Brillouin functional dependence of a Weiss ferromagnet. The shape of the curve depends on the value of $J, J=\frac{1}{2}, 1,2,4$ and $\infty$ respectively, following the direction of the arrow.

was also concluded from the AFM measurements. It is observed that the intensity of the fringes on the left side of the LSMO Bragg peak (small momentum transfer) is higher than that of the fringes on the right side of the peak. This difference is attributed to an elongation of the interfacial unit cells of the LSMO. The inset of the graph shows in grey a simulation of the fringe pattern for a structure with three elongated (by $10 \%$ ) LSMO unit cells at both the interface with the substrate and the surface. The elongation of these interfacial unit cells results in an asymmetry of the fringe pattern with increased intensity at the low momentum transfer side of the pattern. Note that the elongation is expected from the discontinuity in oxygen octahedra rotations at the interface and has been previously observed [30].

\subsection{Functional properties}

The functional properties of LSMO are its magnetization and electrical transport. Here, measurements are presented of an $11.5 \mathrm{~nm}$ LSMO film grown on STO $(001)_{c}$. The sample thickness was determined by counting RHEED oscillations and was confirmed with an XRD reflectivity measurement. The sample has a Curie temperature of $350 \mathrm{~K}$, a saturation magnetization of $4.0 \pm 0.05 \mu_{\mathrm{B}} / \mathrm{Mn}$ and a residual resistivity of $70 \mu \Omega \mathrm{cm}$ at low temperatures $(10 \mathrm{~K})$. 


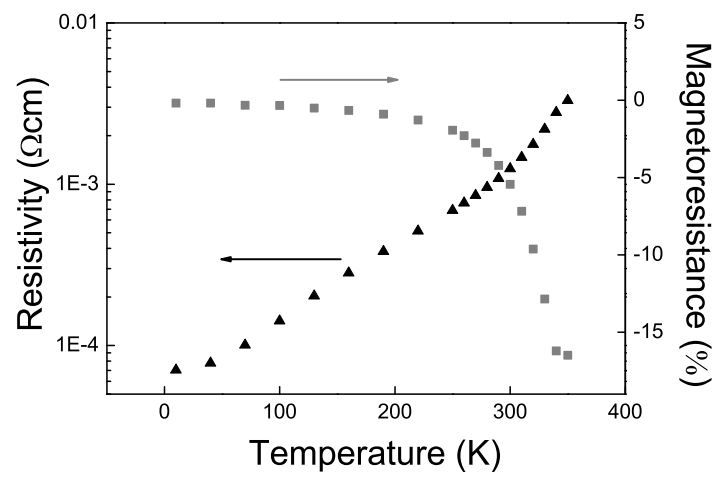

Figure 3.10: Electrical transport measurements of $11.5 \mathrm{~nm}$ LSMO grown on STO $(001)_{c}$. Temperature dependent resistivity and magnetoresistance.

\subsubsection{Magnetization}

The magnetization of the sample was measured with a vibrating sample magnetometer (VSM) (Physical Properties Measurement System (PPMS) by Quantum Design). Figure 3.9a shows the low temperature magnetization loop, obtained at $10 \mathrm{~K}$ with the field applied along the $[100]_{\mathrm{pc}}$ axis and after subtraction of a diamagnetic background from the substrate. The saturation magnetization is reached for an applied field strength larger than $50 \mathrm{kA} / \mathrm{m}$ and is equal to $4.0 \pm 0.05 \mu_{\mathrm{B}} / \mathrm{Mn}$. This implies that next to the expected $3.7 \mu_{\mathrm{B}} / \mathrm{Mn}$ spin angular momentum also 0.3 $\mu_{\mathrm{B}} / \mathrm{Mn}$ orbital angular momentum is present in the LSMO. The magnetization at remanence, $2.7 \mu_{\mathrm{B}} / \mathrm{Mn}$, is significantly smaller than the saturation magnetization. This is partially because the measurement is along the magnetic hard axis [31], and partially because the weak magnetic anisotropy of LSMO on STO results in nanoscale domain formation [32]. The magnetization is very soft with a coercivity of $1.6 \mathrm{kA} / \mathrm{m}$.

The temperature dependence of the magnetization is presented in figure $3.9 \mathrm{~b}$. For each datapoint in the graph, a hysteresis loop between 240 and $-240 \mathrm{kA} / \mathrm{m}$ was measured and the saturation magnetization was calculated after the background subtraction. The Curie temperature of the sample is $350 \mathrm{~K}$. The saturation magnetization follows the Brillouin functional dependence for a Weiss ferromagnet [33]. The curve calculated using $J=4, J$ is the total angular momentum, results in the best fit of the data. This indicates that the spins in the LSMO behave not completely classically (the case of $J=\infty$ ) and not completely quantum mechanically $\left(J=\frac{1}{2}\right)$, but show intermediate behaviour. 


\subsubsection{Electrical transport}

The resistivity of the samples was measured in the van der Pauw configuration [34] (PPMS by Quantum Design). In order to obtain ohmic contacts between the aluminium bonding wires and the LSMO layer, gold contacts were deposited on the corners of the sample with the use of a shadow mask. Measurements were performed as a function of temperature (10-350 K) and magnetic field (0-2.5 T). The temperature dependent resistivity of the sample is shown in figure 3.10. The sample shows metallic behaviour with a residual resistivity at $10 \mathrm{~K}$ of $70 \mu \Omega \mathrm{cm}$. Figure 3.10 also presents the magnetoresistivity as a function of temperature. At each temperature, the magnetic field is swept between -2.5 and $2.5 \mathrm{~T}$ and the magnetoresistivity at $2.5 \mathrm{~T}(\rho(2.5 T)-\rho(0)) / \rho(0)$ is shown. The magnetoresistance is negative and largest, $-16 \%$, at the Curie temperature. Hall effect measurements determined the charge carriers to be holes, as expected for LSMO. The observed Hall coefficient $R_{\mathrm{H}}=4.6 \cdot 10^{-4} \mathrm{~cm}^{3} / \mathrm{C}$ implies a carrier density of approximately 0.8 hole/unit cell, which is in reasonable agreement with earlier measurements on single crystals [35].

\subsection{Photoelectron spectroscopy}

Several LSMO thin films were characterized with in situ x-ray and ultra violet photoelectron spectroscopy (XPS and UPS). The measurements were performed with an XPS/UPS system designed by Omicron Nanotechnology GmbH, equipped with an EA 125 electron energy analyzer. For XPS, an Al K $\alpha$ source (XM 1000) was used and the UV light source is a He plasma lamp (HIS 13) operated at the HeI $(21.22 \mathrm{eV})$ excitation edge. The base pressure of the system was below $10^{-10}$ mbar. The analyzer was calibrated with the use of an in situ sputter cleaned Au sample.

Figure 3.11 shows a survey scan of a 10 unit cell layer LSMO sample grown on $(001)_{\mathrm{c}} \mathrm{STO}$. The main features include the La $3 \mathrm{~d}$ peaks at $850 \mathrm{eV}$, the oxygen 1s peak at $531 \mathrm{eV}$, the Mn 2p peaks at $640 \mathrm{eV}$ and the $\mathrm{Sr}$ and La peaks at low binding energy. Next to this a small Ti peak originating from the substrate can be seen at $460 \mathrm{eV}$. No indications for the presence of impurity atoms, including carbon contamination, was found. This is attributed to the in situ measurement. No attempts have been made to quantify the composition of the film, as large uncertainty margins exist for the element sensitivity factors required to normalize measured peak intensities. From XRD and functional properties measurements it is clear that the composition of the films is equal to the desired LSMO composition within the rather large error margin of the XPS measurements.

The inset of figure 3.11 shows the valence band spectrum as measured with UPS at room temperature. No Fermi edge is observed, the photoelectron count increases linearly with binding energy up to a value of $1.5 \mathrm{eV}$, after which a steep increase is observed. The absence of the Fermi edge is not surprising. In literature, several studies have observed a Fermi edge, but only at low $(<50 \mathrm{~K})$ temperature $[36,37]$. 


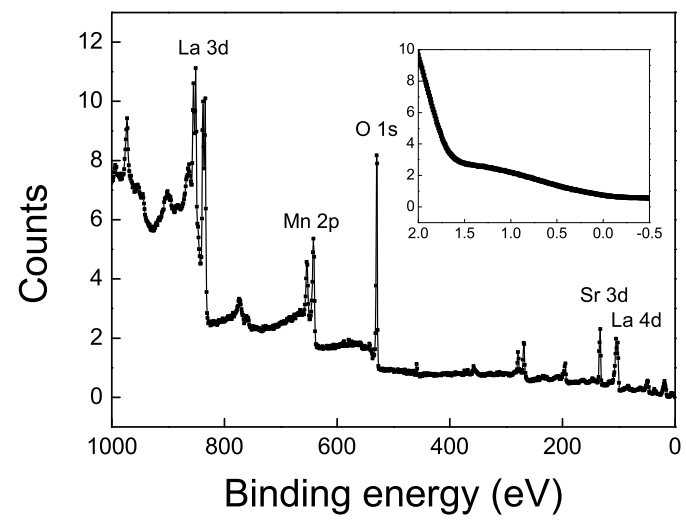

Figure 3.11: An XPS survey scan of a 10 unit cell layer LSMO sample

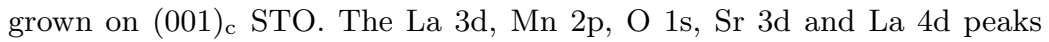
are indicated. The inset shows the photoelectron intensity in the region close to the Fermi energy measured with UPS. The measurements were performed at room temperature.

LSMO's electron density at the Fermi energy is much reduced compared to band theory due to the presence of the pseudogap [37, 38] and disappears above the Curie temperature. It is therefore natural that the Fermi edge cannot easily be observed in a room temperature measurement. The steep increase of the electron density below $1.5 \mathrm{eV}$ is in good agreement with the literature [36].

Several authors have suggested that $\mathrm{Sr}$ or Ca segregation towards the surface of the film is an intrinsic effect in LSMO (or $\mathrm{La}_{0.67} \mathrm{Ca}_{0.33} \mathrm{MnO}_{3}$ (LCMO) respectively) thin films [30, 39]. Angle dependent XPS measurements can be used to study changes in the composition of a thin film in the surface layer. The results obtained for a 10 unit cell layer film grown on $(001)_{c}$ STO are presented in table 3.1. A film thickness of ten unit cell layers was chosen for this experiment in order to directly compare with the 1 to 9 unit cell layer experiments by Herger et al. [30]. If thinner LSMO layers would be used the XPS Sr signal would gain relative intensity due to a substrate contribution, affecting the measurement. As the relative intensity of peaks measured at different take-off angles does not depend on the element sensitivity factors, quantitative analysis is possible. The $\mathrm{Sr} 3 \mathrm{~d}$ peak at $135 \mathrm{eV}$ was compared with the La $4 \mathrm{~d}$ peak at $105 \mathrm{eV}$ and for both elements, an intensity ratio between bulk and surface sensitive measurements of $1.5 \pm 0.05$ was found. This limits the off-stoichiometry of the surface to within $6 \%$. The topmost layer of the literature reference sample has a $\mathrm{La} / \mathrm{Sr}$ ratio of 0.5 instead of 2 , while the subsequent layers are stoichiometric [30]. Based on an electron escape depth of 2 $\mathrm{nm}$ for electrons with kinetic energy of $1350 \mathrm{eV}$ [40], the Herger et al. 9 unit cell layer sample would show an off-stoichiometry of $33 \%$ in the XPS measurement. Therefore, it is concluded that $\mathrm{Sr}$ segregation is diminished in these samples as 
Table 3.1: Integrated XPS peak intensity for a 10 unit cell layer LSMO sample grown on $(001)_{c}$ STO. The XPS intensity is in arbitrary units and for peaks split by e.g. spin orbit coupling the intensities of all the components have been added. The error margins are estimated on the quality of the background subtraction routines.

\begin{tabular}{lcccc}
\hline Peak & Binding energy $(e \mathrm{~V})$ & $\begin{array}{c}10^{\circ} \text { Intensity } \\
\text { (bulk sensitive) }\end{array}$ & $\begin{array}{c}60^{\circ} \text { Intensity } \\
\text { (surface sensitive) }\end{array}$ & Ratio \\
\hline Sr 3d & 135 & $48 \pm 0.5$ & $32 \pm 0.5$ & $1.5 \pm 0.05$ \\
La 4d & 105 & $90 \pm 1$ & $60 \pm 1$ & $1.5 \pm 0.05$ \\
\hline
\end{tabular}

compared to the samples of Herger et al.. It is known that the Sr segregation depends on the oxygen partial pressure [41]. Therefore, the lack of Sr segregation can be attributed to the high oxygen pressure used during the deposition.

XPS was also used to investigate the fine structure of the Mn 2p peak. Typical spectra are shown in figure 3.12. A qualitative difference was observed between the uncapped samples and the samples capped with a 5 unit cell layer of STO. The capped sample shows a sharper 2 p $3 / 2$ peak in comparison with the uncapped sample. In the spectra obtained from the capped sample, a different fine structure can be observed in the Mn 2p 3/2 peak as well. The $3 / 2$ peak actually consists of 2 peaks which are separated by $0.7 \mathrm{eV}$ and the peak at lower binding energy has more intensity. This peak splitting is attributed to the Mn ion multiplet splitting $[42,43]$. For the uncapped sample the $2 \mathrm{p} 3 / 2$ peak is quite broad and significant intensity is observed between the 2 p $3 / 2$ and the 2 p $1 / 2$ peaks. These differences in the spectra indicate that the electronic structure of the capped samples is different. It is suggested that the capping prevents an electronic surface reconstruction, due to the continuation of the oxygen octahedra network, and might result in improved properties. The difference in functional properties between capped and uncapped samples will be discussed in more detail in chapter 5 .

\subsection{Scanning transmission electron microscopy}

A multilayer LSMO/STO sample was characterized with scanning transmission electron microscopy (STEM), to study the interface atomic structure. With STEM an image of the sample can be obtained from the high angle annular dark field (HAADF) intensity measurements while the spectrum of the low angle electrons can be measured (electron energy loss spectroscopy, EELS) for additional chemical information. The STEM data presented was measured with a FEI Titan microscope. The sample is comprised of several layers, from substrate to surface: $4 \mathrm{~nm}$ LSMO, $2 \mathrm{~nm}$ STO, $16 \mathrm{~nm}$ LSMO, $2 \mathrm{~nm}$ STO and $8 \mathrm{~nm}$ LSMO.

Figure 3.13a presents a low magnification HAADF image of the different layers in the sample. In HAADF microscopy, the observed intensity of a column of atoms 


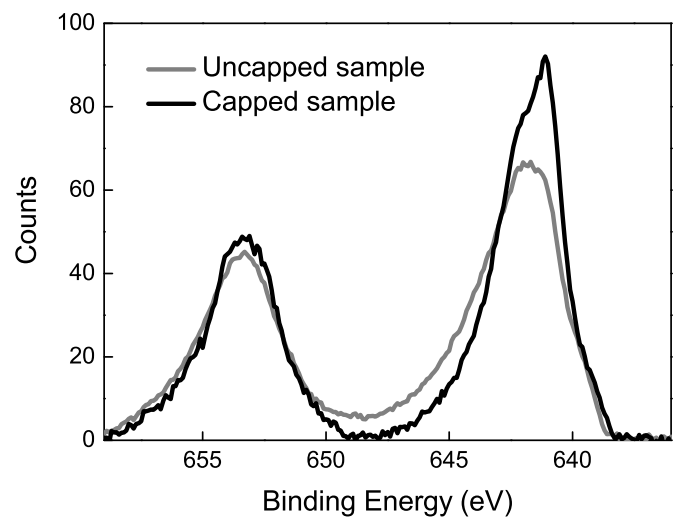

Figure 3.12: Mn 2p spectra of a 30 unit cell uncapped LSMO film and a 13 unit cell LSMO film capped with 5 unit cell layers of STO. After subtraction of a Shirley background, the spectra were normalized to the total intensity in the doublet. The measurements were performed at room temperature.
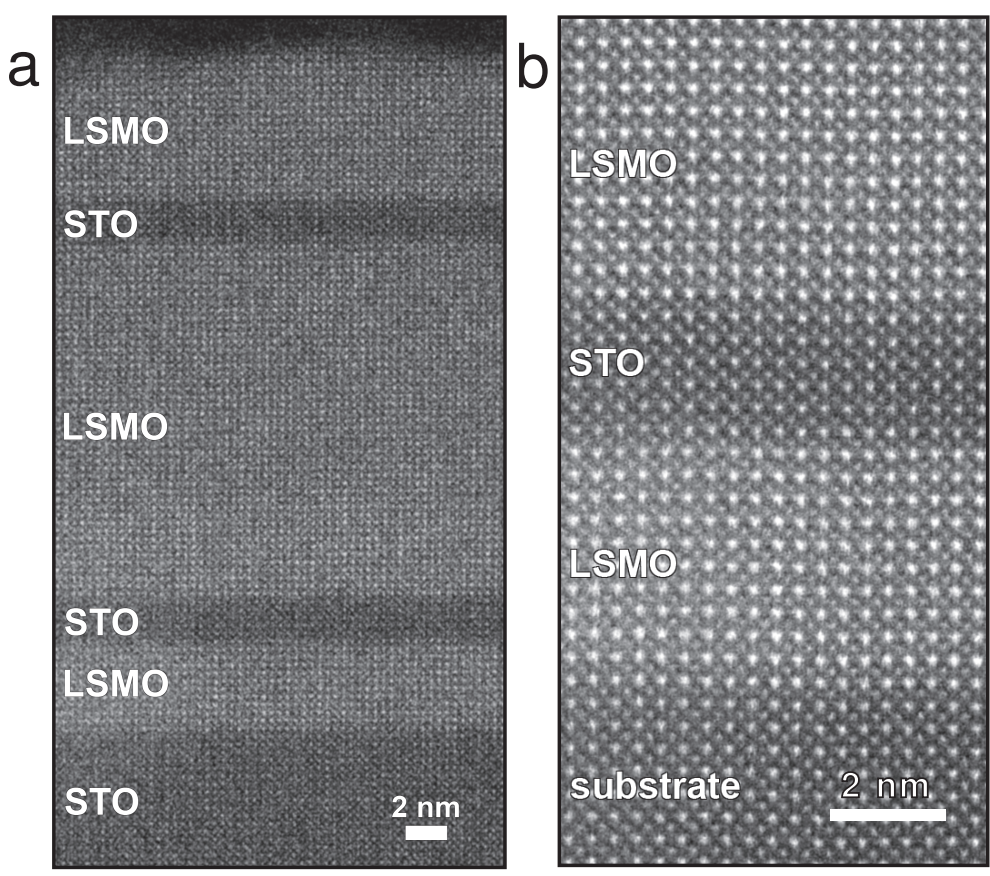

Figure 3.13: HAADF-STEM images of an LSMO/STO heterostructure. a) Low magnification overview. b) High magnification image of the area close to the substrate. These measurements were performed at the EMAT center in Antwerp, Belgium and at the Helmholtz research center Jülich,

Germany. 
scales with the atomic weight of the elements within the columns. Therefore, the LSMO is brighter in the image than the STO. Figure 3.13b presents a higher magnification image obtained from the layers close to the substrate. Both the $A$ and $B$-site columns are observed. The multilayer structure is grown coherently and the interfaces are well defined. A more detailed characterization of the interface structure is presented in chapter 5 .

\subsection{Comparison to other groups}

Table 3.2 presents an overview of the most important properties of LSMO thin films. Data taken from different research groups is included in order to compare the thin film quality. For reference, also the properties of a single crystal are mentioned. All films were grown on STO $(001)_{c}$ substrates by PLD, except for the samples grown in Illinois, for which MBE was used. Most groups published AFM measurements of the surface morphology, which showed smooth surfaces with the step and terrace structure of the substrate clearly visible. Two groups, the Illinois group and the Rome group, did not publish images of the film surface morphology, but they did present a RHEED image from which it was concluded that the films were smooth $[50,54]$. The out-of-plane pseudocubic lattice parameter varies between 3.83 and $3.85 \AA$.

A high quality sample should combine a high $T_{\mathrm{C}}$ with a high saturation magnetization and a low residual resistivity. The samples described in this thesis have the highest saturation magnetization, a high $T_{\mathrm{C}}$ and a low residual resistivity. Only the samples from the Illinois group have a lower residual resistivity while both the Illinois and the Tokyo group obtained a higher $T_{\mathrm{C}}$. Combining all three properties it is concluded that the samples of this work are comparable in quality to the best samples found in the literature.

\subsection{Conclusions}

In this chapter, an overview of the LSMO thin films used in this thesis was given. The films were grown by pulsed laser deposition, monitored with in situ RHEED, and a unit cell layer controlled growth process was achieved. The film growth occurs at first in a predominantly layer-by-layer fashion which changes to the steady state growth mode during deposition, resulting in a smooth surface with islands with unit cell step height. Structural characterization shows that the LSMO films are grown in a fully coherent fashion with respect to the underlying substrates. The characterization of the magnetic and electric transport properties of the films

\footnotetext{
${ }^{1}$ The Rome group mentions that their films have a metal-insulator transition temperature of $370 \mathrm{~K}$. This temperature is defined in their papers as the temperature at which the resistivity of the films has a maximum and it is not equal to the Curie temperature. The value mentioned here is taken from the temperature dependence of the magnetization plot in reference [44]. The data from the 20 unit cell layer sample was used, which has a similar metal-insulator transition temperature as their thick samples.
} 
Table 3.2: Overview of the thin film properties. $T_{\mathrm{C}}$ is the Curie temperature, $\rho_{0}$ is the residual resistivity at $10 \mathrm{~K}$ and $M_{\text {sat }}$ is the saturation magnetization at $10 \mathrm{~K}$.

\begin{tabular}{|c|c|c|c|}
\hline Group & {$[001]_{\mathrm{pc}}$ axis $(\AA)$} & $T_{\mathrm{C}}(\mathrm{K})$ & Remarks \\
\hline Deposition & $\rho_{0}(\mu \Omega \mathrm{cm})$ & $M_{\mathrm{sat}}\left(\mu_{\mathrm{B}} / \mathrm{Mn}\right)$ & References \\
\hline Tokyo (Tokura et al.) & - & 370 & - \\
\hline Single crystal & 90 & 3.5 & {$[45]$} \\
\hline Tsukuba (Kawasaki et al.) & 3.83 & 340 & - \\
\hline PLD & 200 & 3.6 & {$[46-49]$} \\
\hline Illinois (Eckstein et al.) & 3.85 & 355 & - \\
\hline $\mathrm{MBE}$ & 40 & 3.2 & {$[6,50,51]$} \\
\hline Orsay Cedex (Contour et al.) & - & 350 & - \\
\hline PLD & - & 3.7 & {$[8,52,53]$} \\
\hline Rome (Balestrino et al.) & 3.85 & 300 & see footnote ${ }^{1}$ \\
\hline PLD & 1000 & 3.5 & {$[44,54,55]$} \\
\hline Tokyo (Hwang et al.) & 3.84 & 360 & - \\
\hline PLD & 60 & - & {$[56-58]$} \\
\hline Twente (This work) & 3.845 & 350 & - \\
\hline PLD & 60 & 4.0 & {$[26]$} \\
\hline
\end{tabular}


show a saturation magnetization of $4.0 \mu_{\mathrm{B}} / \mathrm{Mn}$, a Curie temperature of $350 \mathrm{~K}$ and a residual resistivity of $70 \mu \Omega \mathrm{cm}$. XPS measurements show the absence of chemical impurities and no evidence for surface segregation. STEM measurements show that multi-layer structures with sharp interfaces were realized. All results indicate that high quality films, comparable to the best examples found in literature, were fabricated. The ability to grow high quality films and multilayer structures is an important prerequisite for the research in the later chapters in this thesis. 


\subsection{References}

[1] R. von Helmolt, J. Wecker, B. Holzapfel, L. Schultz, and K. Samwer, "Giant negative magnetoresistance in perovskitelike $\mathrm{La}_{2 / 3} \mathrm{Ba}_{1 / 3} \mathrm{MnO}_{x}$

ferromagnetic-films," PHYSICAL REVIEW LETTERS, vol. 71, pp. 2331-2333, 1993.

[2] S. Jin, T. Tiefel, M. Mccormack, R. Fastnacht, R. Ramesh, and L. Chen, "Thousandfold change in resistivity in magnetoresistive La-Ca-Mn-O films," SCIENCE, vol. 264, pp. 413-415, 1994.

[3] H. Hwang, S. Cheong, N. Ong, and B. Batlogg, "Spin-polarized intergrain tunneling in $\mathrm{La}_{2 / 3} \mathrm{Sr}_{1 / 3} \mathrm{MnO}_{3}$," PHYSICAL REVIEW LETTERS, vol. 77, pp. 2041-2044, 1996.

[4] N. Mathur, G. Burnell, S. Isaac, T. Jackson, B. Teo, J. MacManusDriscoll, L. Cohen, J. Evetts, and M. Blamire, "Large low-field magnetoresistance in $\mathrm{La}_{0.7} \mathrm{Ca}_{0.3} \mathrm{MnO}_{3}$ induced by artificial grain boundaries," NATURE, vol. 387, pp. 266-268, 1997.

[5] J. Sun, W. Gallagher, P. Duncombe, L. KrusinElbaum, R. Altman, A. Gupta, Y. Lu, G. Gong, and G. Xiao, "Observation of large low-field magnetoresistance in trilayer perpendicular transport devices made using doped manganate perovskites," APPLIED PHYSICS LETTERS, vol. 69, pp. 3266-3268, 1996.

[6] J. O'Donnell, A. Andrus, S. Oh, E. Colla, and J. Eckstein, "Colossal magnetoresistance magnetic tunnel junctions grown by molecular-beam epitaxy," APPLIED PHYSICS LETTERS, vol. 76, pp. 1914-1916, 2000.

[7] Y. Ogimoto, M. Izumi, A. Sawa, T. Manako, H. Sato, H. Akoh, M. Kawasaki, and Y. Tokura, "Tunneling magnetoresistance above room temperature in $\mathrm{La}_{0.7} \mathrm{Sr}_{0.3} \mathrm{MnO}_{3} / \mathrm{SrTiO}_{3} / \mathrm{La}_{0.7} \mathrm{Sr}_{0.3} \mathrm{MnO}_{3}$ junctions," JAPANESE JOURNAL OF APPLIED PHYSICS PART 2-LETTERS, vol. 42, pp. L369-L372, 2003.

[8] M. Bowen, M. Bibes, A. Barthelemy, J. Contour, A. Anane, Y. Lemaitre, and A. Fert, "Nearly total spin polarization in $\mathrm{La}_{2 / 3} \mathrm{Sr}_{1 / 3} \mathrm{MnO}_{3}$ from tunneling experiments," APPLIED PHYSICS LETTERS, vol. 82, pp. 233-235, 2003.

[9] Y. Tokura, "Features of cmr manganites," in Colossal magnetoresistive oxides (Y. Tokura, ed.), pp. 1-52, Gordon and Breach Science Publishers, the Netherlands, 2000.

[10] J. Goodenough, "An interpretation of the magnetic properties of the perovskite-type mixed crystals $\mathrm{La}_{1-\mathrm{x}} \mathrm{Sr}_{\mathrm{x}} \mathrm{CoO}_{3-\lambda}$," JOURNAL OF PHYSICS AND CHEMISTRY OF SOLIDS, vol. 6, pp. 287-297, 1958.

[11] J. Kanamori, "Superexchange interaction and symmetry properties of electron orbitals," JOURNAL OF PHYSICS AND CHEMISTRY OF SOLIDS, vol. 10, pp. 87-98, 1959. 
[12] C. Zener, "Interaction between the d-shells in the transition metals .2. ferromagnetic compounds of manganese with perovskite structure," PHYSICAL REVIEW, vol. 82, pp. 403-405, 1951.

[13] P. Anderson and H. Hasegawa, "Considerations on double exchange," PHYSICAL REVIEW, vol. 100, pp. 675-681, 1955.

[14] P. DeGennes, "Effect of double exchange in magnetic crystals," PHYSICAL REVIEW, vol. 118, pp. 141-154, 1960.

[15] H. Fujishiro, T. Fukase, and M. Ikebe, "Charge ordering and sound velocity anomaly in $\mathrm{La}_{1-x} \mathrm{Sr}_{x} \mathrm{MnO}_{3}(\mathrm{X}>=0.5)$," JOURNAL OF THE PHYSICAL SOCIETY OF JAPAN, vol. 67, pp. 2582-2585, 1998.

[16] H. Fujishiro, M. Ikebe, Y. Konno, and T. Fukase, "Sound velocity anomaly associated with polaron ordering in $\mathrm{La}_{1-x} \mathrm{Sr}_{x} \mathrm{MnO}_{3}$," JOURNAL OF THE PHYSICAL SOCIETY OF JAPAN, vol. 66, pp. 3703-3705, 1997.

[17] J. Rodriguez-Carvajal, M. Hennion, F. Moussa, A. Moudden, L. Pinsard, and A. Revcolevschi, "Neutron-diffraction study of the Jahn-Teller transition in stoichiometric $\mathrm{LaMnO}_{3}$," PHYSICAL REVIEW B, vol. 57, pp. R3189-R3192, 1998 .

[18] H. Hwang, S. Cheong, P. Radaelli, M. Marezio, and B. Batlogg, "Lattice effects on the magnetoresistance in doped $\mathrm{LaMnO}_{3}$," PHYSICAL REVIEW LETTERS, vol. 75 , pp. 914-917, 1995.

[19] P. Radaelli, G. Iannone, M. Marezio, H. Hwang, S. Cheong, J. Jorgensen, and D. Argyriou, "Structural effects on the magnetic and transport properties of perovskite $\mathrm{A}_{(1-x)} \mathrm{A}_{(x)} \mathrm{MnO}_{3}(\mathrm{x}=0.25,0.30)$," PHYSICAL REVIEW B, vol. 56, pp. 8265-8276, 1997.

[20] S. W. Cheong and H. Y. Hwang, "Ferromagnetism vs. charge/orbital ordering in mixed-valent manganites," in Colossal magnetoresistive oxides (Y. Tokura, ed.), pp. 237-280, Gordon and Breach Science Publishers, the Netherlands, 2000.

[21] G. Koster, B. Kropman, G. Rijnders, D. Blank, and H. Rogalla, "Quasi-ideal strontium titanate crystal surfaces through formation of strontium hydroxide," APPLIED PHYSICS LETTERS, vol. 73, pp. 2920-2922, 1998.

[22] W. Siemons, Nanoscale properties of complex oxide films. PhD thesis, University of Twente, 2008.

[23] R. Bachelet, F. Sanchez, F. J. Palomares, C. Ocal, and J. Fontcuberta, "Atomically flat $\mathrm{SrO}$-terminated $\mathrm{SrTiO}_{3}(001)$ substrate," APPLIED PHYSICS LETTERS, vol. 95, p. 141915, 2009.

[24] G. Koster, G. Rijnders, D. Blank, and H. Rogalla, "Surface morphology determined by (001) single-crystal $\mathrm{SrTiO}_{3}$ termination," PHYSICA C-SUPERCONDUCTIVITY AND ITS APPLICATIONS, vol. 339, pp. 215-230, 2000 .

[25] M. Mathews, Structural and magnetic properties of epitaxial $\mathrm{La}_{0.67} \mathrm{Sr}_{0.33} \mathrm{MnO}_{3}$ films and nanostructures. PhD thesis, University of Twente, 2007. 
[26] M. Huijben, L. W. Martin, Y. H. Chu, M. B. Holcomb, P. Yu, G. Rijnders, D. H. A. Blank, and R. Ramesh, "Critical thickness and orbital ordering in ultrathin $\mathrm{La}_{0.7} \mathrm{Sr}_{0.3} \mathrm{MnO}_{3}$ films," PHYSICAL REVIEW B, vol. 78, p. 094413, 2008.

[27] T. Higuchi, T. Yajima, L. F. Kourkoutis, Y. Hikita, N. Nakagawa, D. A. Muller, and H. Y. Hwang, " $\mathrm{Mn}_{3} \mathrm{O}_{4}$ precipitates in laser-ablated manganite films," APPLIED PHYSICS LETTERS, vol. 95, p. 043112, 2009.

[28] N. Farag, M. Bobeth, W. Pompe, A. Romanov, and J. Speck, "Modeling of twinning in epitaxial (001)-oriented $\mathrm{La}_{0.67} \mathrm{Sr}_{0.33} \mathrm{MnO}_{3}$ thin films," JOURNAL OF APPLIED PHYSICS, vol. 97, p. 113516, 2005.

[29] A. Vailionis, H. Boschker, W. Siemons, E. P. Houwman, G. Rijnders, D. H. A. Blank, and G. Koster, "Misfit strain accommodation in epitaxial $\mathrm{ABO}_{3}$ perovskites: lattice rotations and lattice modulations," arXiv:1009.6018v1.

[30] R. Herger, P. R. Willmott, C. M. Schlepuetz, M. Bjoerck, S. A. Pauli, D. Martoccia, B. D. Patterson, D. Kumah, R. Clarke, Y. Yacoby, and M. Doebeli, "Structure determination of monolayer-by-monolayer grown $\mathrm{La}_{1-x} \mathrm{Sr}_{x} \mathrm{MnO}_{3}$ thin films and the onset of magnetoresistance," PHYSICAL REVIEW B, vol. 77, p. 085401, 2008.

[31] M. Mathews, F. Postma, J. Lodder, R. Jansen, G. Rijnders, and D. Blank, "Step-induced uniaxial magnetic anisotropy of $\mathrm{La}_{0.67} \mathrm{Sr}_{0.33} \mathrm{MnO}_{3}$ thin films," APPLIED PHYSICS LETTERS, vol. 87, p. 242507, 2005.

[32] E. P. Houwman, G. Maris, G. M. De Luca, N. Niermann, G. Rijnders, D. H. A. Blank, and S. Speller, "Out-of-plane magnetic domain structure in a thin film of $\mathrm{La}_{0.67} \mathrm{Sr}_{0.33} \mathrm{MnO}_{3}$ on $\mathrm{SrTiO}_{3}$ (001) observed by magnetic force microscopy," PHYSICAL REVIEW B, vol. 77, p. 184412, 2008.

[33] S. Chikazumi, Physics of ferromagnetism. John Wiley and Sons, Inc, New York, 1964 .

[34] L. van der Pauw, "A method of measuring the resistivity and Hall coefficient on lamellae of arbitrary shape," Philips Technical Review, vol. 20, pp. 220-224, 1958.

[35] A. Asamitsu and Y. Tokura, "Hall effect in $\mathrm{La}_{1-x} \mathrm{Sr}_{x} \mathrm{MnO}_{3}$, " PHYSICAL REVIEW B, vol. 58, pp. 47-50, 1998.

[36] J. Park, E. Vescovo, H. Kim, C. Kwon, R. Ramesh, and T. Venkatesan, "Direct evidence for a half-metallic ferromagnet," NATURE, vol. 392, pp. 794-796, 1998.

[37] D. S. Dessau and Z. X. Shen, "Direct electronic structure measurements of the colossal magnetoresistive oxides," in Colossal magnetoresistive oxides (Y. Tokura, ed.), pp. 149-185, Gordon and Breach Science Publishers, the Netherlands, 2000.

[38] Y. Chuang, A. Gromko, D. Dessau, T. Kimura, and Y. Tokura, "Fermi surface nesting and nanoscale fluctuating charge/orbital ordering in colossal magnetoresistive oxides," SCIENCE, vol. 292, pp. 1509-1513, 2001.

[39] J. Simon, T. Walther, W. Mader, J. Klein, D. Reisinger, L. Alff, and R. Gross, "Diffusion and segregation effects in doped manganite/titanate heterostructures," APPLIED PHYSICS LETTERS, vol. 84, pp. 3882-3884, 2004. 
[40] NIST Standard Reference Database 71, NIST Electron Inelastic-Mean-Free-Path Database: Ver. 1.1, URL http://www.nist.gov/srd/nist71.htm.

[41] T. T. Fister, D. D. Fong, J. A. Eastman, P. M. Baldo, M. J. Highland, P. H. Fuoss, K. R. Balasubramaniam, J. C. Meador, and P. A. Salvador, "In situ characterization of strontium surface segregation in epitaxial $\mathrm{La}_{0.7} \mathrm{Sr}_{0.3} \mathrm{MnO}_{3}$ thin films as a function of oxygen partial pressure," APPLIED PHYSICS LETTERS, vol. 93, p. 151904, 2008.

[42] R. Gupta and S. Sen, "Calculation of multiplet structure of core p-vacancy levels," PHYSICAL REVIEW B, vol. 10, pp. 71-77, 1974.

[43] R. Gupta and S. Sen, "Calculation of multiplet structure of core p-vacancy levels .2," PHYSICAL REVIEW B, vol. 12, pp. 15-19, 1975.

[44] A. Tebano, C. Aruta, S. Sanna, P. G. Medaglia, G. Balestrino, A. A. Sidorenko, R. De Renzi, G. Ghiringhelli, L. Braicovich, V. Bisogni, and N. B. Brookes, "Evidence of orbital reconstruction at interfaces in ultrathin $\mathrm{La}_{0.67} \mathrm{Sr}_{0.33} \mathrm{MnO}_{3}$ films," PHYSICAL REVIEW LETTERS, vol. 100, p. 137401, 2008.

[45] A. Urushibara, Y. Moritomo, T. Arima, A. Asamitsu, G. Kido, and Y. Tokura, "Insulator-metal transition transition and giant magnetoresistance in $\mathrm{La}_{1-x} \mathrm{Sr}_{x} \mathrm{MnO}_{3}, "$ PHYSICAL REVIEW B, vol. 51, pp. 14103-14109, 1995.

[46] M. Izumi, Y. Konishi, T. Nishihara, S. Hayashi, M. Shinohara, M. Kawasaki, and Y. Tokura, "Atomically defined epitaxy and physical properties of strained $\mathrm{La}_{0.6} \mathrm{Sr}_{0.4} \mathrm{MnO}_{3}$ films," APPLIED PHYSICS LETTERS, vol. 73, pp. 2497-2499, 1998.

[47] H. Yamada, Y. Ogawa, Y. Ishii, H. Sato, M. Kawasaki, H. Akoh, and Y. Tokura, "Engineered interface of magnetic oxides," SCIENCE, vol. 305, pp. 646-648, 2004.

[48] K. Horiba, A. Chikamatsu, H. Kumigashira, M. Oshima, N. Nakagawa, M. Lippmaa, K. Ono, M. Kawasaki, and H. Koinuma, "In vacuo photoemission study of atomically controlled $\mathrm{La}_{1-x} \mathrm{Sr}_{x} \mathrm{MnO}_{3}$ thin films: Composition dependence of the electronic structure," PHYSICAL REVIEW B, vol. 71, p. 155420, 2005.

[49] H. Yamada, M. Kawasaki, T. Lottermoser, T. Arima, and Y. Tokura, " $\mathrm{LaMnO}_{3} / \mathrm{SrMnO}_{3}$ interfaces with coupled charge-spin-orbital modulation," APPLIED PHYSICS LETTERS, vol. 89, p. 052506, 2006.

[50] J. J. Kavich, M. P. Warusawithana, J. W. Freeland, P. Ryan, X. Zhai, R. H. Kodama, and J. N. Eckstein, "Nanoscale suppression of magnetization at atomically assembled manganite interfaces: XMCD and XRMS measurements," PHYSICAL REVIEW B, vol. 76, p. 014410, 2007.

[51] A. Bhattacharya, S. J. May, S. G. E. t. Velthuis, M. Warusawithana, X. Zhai, B. Jiang, J. M. Zuo, M. R. Fitzsimmons, S. D. Bader, and J. N. Eckstein, "Metal-insulator transition and its relation to magnetic structure in $\left(\mathrm{LaMnO}_{3}\right)_{(2 n)} /\left(\mathrm{SrMnO}_{3}\right)_{(n)}$ superlattices," PHYSICAL REVIEW LETTERS, vol. 100, p. 257203, 2008.

[52] J. Maurice, F. Pailloux, A. Barthelemy, A. Rocher, O. Durand, R. Lyonnet, and J. Contour, "Strain and magnetism in $\left(\mathrm{La}_{0.7} \mathrm{Sr}_{0.3}\right) \mathrm{MnO}_{3}$ very thin films epitaxially grown on $\mathrm{SrTiO}_{3}$," APPLIED SURFACE SCIENCE, vol. 188, pp. 176-181, 2002. 
[53] F. Pailloux, D. Imhoff, T. Sikora, A. Barthelemy, J. Maurice, J. Contour, C. Colliex, and A. Fert, "Nanoscale analysis of a $\mathrm{SrTiO}_{3} / \mathrm{La}_{2 / 3} \mathrm{Sr}_{1 / 3} \mathrm{MnO}_{3}$ interface," PHYSICAL REVIEW B, vol. 66, p. 014417, 2002.

[54] A. Tebano, G. Balestrino, N. Boggio, C. Aruta, B. Davidson, and P. Medaglia, "High-quality in situ manganite thin films by pulsed laser deposition at low background pressures," EUROPEAN PHYSICAL JOURNAL B, vol. 51, pp. 337-340, 2006.

[55] C. Aruta, G. Balestrino, A. Tebano, G. Ghiringhelli, and N. B. Brookes, "Cooperative enhancement of in-plane orbital ordering by oxygen deficiency and in-plane tensile strain in $\mathrm{La}_{0.7} \mathrm{Sr}_{0.3} \mathrm{MnO}_{3-\delta}$ thin films," $E P L$, vol. 80, p. 37003, 2007.

[56] J. H. Song, T. Susaki, and H. Y. Hwang, "Enhanced thermodynamic stability of epitaxial oxide thin films," ADVANCED MATERIALS, vol. 20, pp. 2528+, 2008.

[57] B. Kim, D. Kwon, J. H. Song, Y. Hikita, B. G. Kim, and H. Y. Hwang, "Finite size effect and phase diagram of ultra-thin $\mathrm{La}_{0.7} \mathrm{Sr}_{0.3} \mathrm{MnO}_{3}$," SOLID STATE COMMUNICATIONS, vol. 150, pp. 598-601, 2010.

[58] L. F. Kourkoutis, J. H. Song, H. Y. Hwang, and D. A. Muller, "Microscopic origins for stabilizing room-temperature ferromagnetism in ultrathin manganite layers," PROCEEDINGS OF THE NATIONAL ACADEMY OF SCIENCES OF THE UNITED STATES OF AMERICA, vol. 107, pp. 11682-11685, 2010. 


\title{
Chapter 4
}

\section{Magnetocrystalline anisotropy in strained $\mathbf{L a}_{0.67} \mathbf{S r}_{0.33} \mathrm{MnO}_{3}$ thin films}

\begin{abstract}
In $\mathrm{La}_{0.67} \mathrm{Sr}_{0.33} \mathrm{MnO}_{3}$ (LSMO) thin films, the crystal structure is determined by the strain from the underlying substrate. With the choice of a specific substrate surface, the magnetic properties of the LSMO layer can be controlled. A model, based on the framework of Néel, was developed which predicts the magnetocrystalline anisotropy. The model was verified with measurements of the magnetic anisotropy of LSMO thin films grown on different surfaces of $\mathrm{NdGaO}_{3}$ single crystal substrates. The experiments uniquely demonstrate that both the Néel parameters $L_{0}$ and $L^{\prime}$ are negative. The origin of the anisotropy is the alignment of the Mn orbitals in the LSMO crystal field. The physical meaning of a negative $L_{0}$ is that the shape of the orbitals is oblate. The crystal field due to the neighbouring oxygen ions was evaluated with respect to strain. As $L^{\prime}$ was found to be negative as well, the analysis of the crystal field interaction suggests that the angles of the $\mathrm{MnO}_{6}$ octahedra change with strain while the Mn-O bond lengths remain approximately constant.
\end{abstract}

\subsection{Introduction}

With the development of high quality oxide heteroepitaxy, strain engineering has become possible for complex oxide materials. When a material is epitaxially strained to a substrate, the in-plane lattice vectors of the materials unit cell are matched to those of the substrate and the functional properties of the material may 
be different from the bulk. So strain engineering can be used to enhance or tailor the properties of the materials. In the field of ferroelectrics, where the functional properties are directly related to the materials' crystal structure, a number of interesting examples can be found. Choi et al. found that the polarization and ferroelectric transition temperature of $\mathrm{BaTiO}_{3}$ can be increased significantly if the material is compressively strained [1]. Haeni et al. showed that paraelectric $\mathrm{SrTiO}_{3}$ can be turned into a ferroelectric with the use of strain engineering [2]. Strain engineering can also be used to control the direction of the polarization as shown by Catalan et al., who measured in-plane instead of out-of-plane polarization when $\mathrm{PbTiO}_{3}$ was subjected to tensile strain [3].

In the field of ferromagnetic materials, one of the functional properties is directly related to the crystal structure as well, namely the magnetocrystalline anisotropy. It determines the ferromagnet's preferential magnetization directions as well as the magnetization reversal mechanism and therefore the possibility to engineer this anisotropy can be extremely useful. The magnetization of $\mathrm{La}_{0.67} \mathrm{Sr}_{0.33} \mathrm{MnO}_{3}$ (LSMO) is very sensitive to epitaxial strain and can be ninety degrees rotated by small changes in the substrates' in-plane lattice parameters. Control of the magnetization direction is a requirement for the operation of LSMO spintronic and multiferroic devices. In these devices, a change in the magnetization of the LSMO is affected by either an applied magnetic field [4-6], a change in the strain state due to strain coupling with a ferro-/piezoelectric material $[7,8]$ or exchange coupling to a switchable ferroelectric-antiferromagnetic multiferroic such as $\mathrm{BiFeO}_{3}$ [9]. For all of these devices, a specific magnetic anisotropy of the LSMO is required for optimum performance. For example, Eerenstein et al [8] demonstrated that the amount of magnetization in the LSMO can be reversibly changed based on the amount of strain applied with a piezoelectric material. Ideally, the amount of strain from the piezoelectric material should not control the amount but the direction of the magnetization, so that the magnetization can be switched between two orthogonal directions. Therefore, understanding of the relation between the crystal structure and the magnetocrystalline anisotropy of LSMO is required.

Bulk LSMO has a distorted cubic crystal structure, in which the Mn ions are at the corners of the cube and the $\mathrm{La} / \mathrm{Sr}$ ions are in the centre. The $\mathrm{O}$ ions form octahedra around the $\mathrm{Mn}$ ions, so each $\mathrm{Mn}$ ion has six nearest neighbours. The distortion from the cubic structure is a shortening of one of the body diagonals, resulting in a rhombohedral structure $[10,11]$. The magnetocrystalline anisotropy is uniaxial, with either an easy axis aligned with the shortened $[111]_{p c}$ lattice vector, as proposed by Konoto et al. [12], or an easy plane perpendicular to the shortened $[111]_{p c}$ lattice vector, as proposed by Khapikov et al. [13] and Steenbeck et al. [14]. This issue is still unresolved.

For LSMO thin films grown on substrates with different lattice parameters, the anisotropy was found to be out-of-plane for compressive strain $\left(\mathrm{LaAlO}_{3}(\mathrm{LAO})\right.$, LSAT and NGO) and in-plane for tensile strain (STO) [15-17]. Stripe domains, observed with magnetic force microscopy in LSMO samples grown on (110) o NGO, indicate an in-plane uniaxial anisotropy (together with an out-of-plane component) [17], but no relation to a specific crystal direction was mentioned. For LSMO grown 
on $(001)_{c}$ STO, both uniaxial and biaxial in-plane anisotropy have been found [18-21]. The uniaxial contribution is caused by stepedge induced anisotropy and the biaxial anisotropy is magnetocrystalline in origin. The biaxial anisotropy has easy axes in the $\langle 110\rangle_{\mathrm{pc}}$ directions of the LSMO. The rich variety in anisotropy, stepedge induced, uniaxial and biaxial, necessitates a model which predicts the anisotropy for a given substrate surface.

Here, a model for the magnetocrystalline anisotropy of LSMO is developed, both for the bulk and for LSMO strained to different substrate surfaces. The model can be used to obtain easy and hard axes and the anisotropy constants. The framework of Néel [22] is used, where the model input consists of the lattice constants of the material and three phenomelogical parameters. With examples of specific crystal structure distortions, in which the anisotropy energy depends only on one of the Néel parameters, the effect of strain on the magnetic properties is illustrated. The model can easily be extended to include surface and stepedge anisotropy.

The model predictions are compared to experimental results obtained from strained LSMO thin films. By growing LSMO on different substrates or substrates with different surface plane orientations, the amount of strain can be controlled. For the experiments, $\mathrm{NdGaO}_{3}$ was chosen as a substrate. The NGO unit cell is orthorhombic and by using different surface plane orientations, both the distances and the angles of the LSMO unit cell are changed. NGO substrate surfaces are suitable for anisotropy studies, as the crystal distortions corresponding to a single Néel parameter can be obtained. Therefore, the experiments can directly be compared to the model.

The origin of magnetocrystalline anisotropy is a combination of spin orbit coupling and crystal field interaction [23]. By comparing the Néel parameters with the model of single ion anisotropy, it is shown that the electron clouds in LSMO are oblate in contrast to the prolate electron clouds in parent compound $\mathrm{LaMnO}_{3}$ (LMO) [24]. It is suggested, that the main contribution to the anisotropy in LMO is due to prolate $\mathrm{e}_{g}$ orbitals, while the main contribution to the anisotropy of LSMO is due to oblate $\mathrm{t}_{2 g}$ orbitals. When strain is applied to a single Mn-Mn bond, the analysis predicts that the angles of the $\mathrm{MnO}_{6}$ octahedra change, while the Mn-O bond lengths remain approximately constant.

This chapter is organized as follows. First, the Néel model is discussed. Then, the experimental results are presented and analyzed within the framework of the Néel model. From this analysis, the signs of the Néel model parameters are obtained, which are used in the final section to determine the structural response of the oxygen octahedra to the applied strain in the analysis of the single ion anisotropy model.

\subsection{Néel framework}

In this section, the Néel model as used in this chapter is discussed. The model predicts the anisotropy energy based on the material's crystal structure and three 
phenomelogical constants. Several examples are presented, which show the effect of the main distortions of a cubic crystal structure. These results will be used in the experimental section to describe the anisotropy of strained LSMO films.

\subsubsection{Model}

The Néel model for magnetic anisotropy was developed by Louis Néel in 1954 [22]. He considered the magnetic pair interaction between two magnetic ions. Even though originally inspired by magnetostatic dipole dipole interactions, which are known to be too weak to explain the anisotropy, the formalism is quite useful. The energy of a single pair is given by:

$$
E_{i j}\left(r_{i j}, \zeta\right)=G\left(r_{i j}\right)+L\left(r_{i j}\right)\left(\cos ^{2}(\zeta)-\frac{1}{3}\right)+Q\left(r_{i j}\right)\left(\cos ^{4}(\zeta)-\frac{6}{7} \cos ^{2}(\zeta)-\frac{3}{35}\right),
$$

in which $r_{i j}$ is the vector between the spins, $\zeta$ is the angle between the bond vector and the magnetization and $G\left(r_{i j}\right), L\left(r_{i j}\right), Q\left(r_{i j}\right)$ are the isotropic, dipolar and quadrupolar Néel parameters. The first term does not give rise to anisotropy as it does not depend on $\zeta$. The second term describes the uniaxial anisotropy and the third term the biaxial anisotropy. Typically $Q\left(r_{i j}\right)$ is a few orders of magnitude smaller than $L\left(r_{i j}\right)$ [25].

To calculate the magnetocrystalline anisotropy, a summation of equation 4.1 over the spin pairs in the unit cell has to be made. Here, only nearest neighbour interactions are taken into account. The parameters in the equation depend on the bond length and they can be approximated around an equilibrium value $r_{0}$. The interaction coefficient $L\left(r_{i j}\right)$ can then be written as:

$$
L\left(r_{i j}\right)=L_{0}+L^{\prime} \epsilon,
$$

in which $L_{0}$ is $L\left(r_{0}\right), L^{\prime}$ is $\mathrm{d} L / \mathrm{d} r_{i j}\left(r_{0}\right) \cdot r_{0}$ and $\epsilon=\left(r_{i j}-r_{0}\right) / r_{0}$, which is the normalized strain. The quadrupolar parameter $Q\left(r_{i j}\right)$ is approximated with a constant $Q_{0}=Q\left(r_{0}\right)$ [26]. The energy of a single bond with strain $\epsilon$ depends on the magnetization angle and the three independent parameters $L_{0}, L^{\prime}$ and $Q_{0}$.

For LSMO three bonds are present in the (pseudocubic) unit cell. The direction of the bonds is described with the unit vector $\boldsymbol{a}_{i j}$. Here, a coordinate system is used, in which $z$ corresponds to the out-of-plane direction of the thin film and $x$ and $y$ are in the plane of the film. The magnetization is defined by the vector $\boldsymbol{M}_{\boldsymbol{s}}=(\sin \theta \cos \phi, \sin \theta \sin \phi, \cos \theta)$, where $\theta$ is the angle of $\boldsymbol{M}_{\boldsymbol{s}}$ with the positive z-axis and $\phi$ the counter clockwise angle with the in-plane positive x-axis. The angle $\zeta$ in equation 4.1 can be calculated from the inner product of $\boldsymbol{M}_{\boldsymbol{s}}$ and $\boldsymbol{a}_{i j}$.

\subsubsection{Distortions of the cubic lattice}

First, the anisotropy of a simple cubic lattice with unstrained bonds is calculated. As all bond lengths are equal and the bonds are $90^{\circ}$ with respect to each other, 

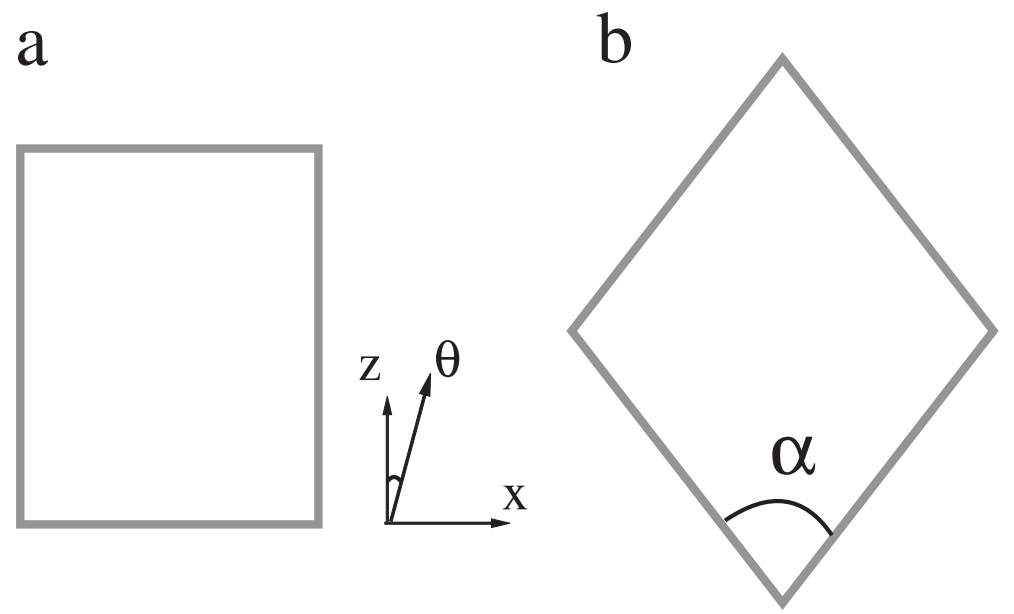

Figure 4.1: Schematics of the distorted cubic crystal structures, as used in the model calculations. a) Side view of the strained bond length structure.

b) Side view of the strained bond angle structure.

all dipolar contributions cancel. The only angular dependent term in the energy is due to the quadrupolar term $Q_{0}$. For $Q_{0}>0$, this results in four equivalent easy axes along the $\langle 111\rangle_{c}$ body diagonals. For $Q_{0}<0$, three easy axes are present, aligned with the $\langle 100\rangle_{c}$ crystal directions.

\section{Strained bond lengths}

The general distortion of the simple cubic lattice encountered in $(001)_{\mathrm{pc}}$ oriented thin films is the elongation/shortening of the out-of-plane lattice parameter, which is shown in figure 4.1a. As the quadrupolar term is much smaller than the dipolar term, the dominant contribution to the anisotropy is the unit cell elongation/shortening. The anisotropy energy is then:

$$
E=\Delta \epsilon L^{\prime} \cos ^{2} \theta
$$

in which $\Delta \epsilon=\frac{r_{\mathrm{z}}-r_{\mathrm{x}}}{r_{0}}$ is the difference in strain between the in-plane and out-ofplane lattice parameters. For an elongated unit cell, the magnetization will be outof-plane when $L^{\prime}<0$ and in-plane when $L^{\prime}>0$. For a unit cell with a shortened lattice parameter in the out-of-plane direction, the magnetization will be in-plane for $L^{\prime}<0$ and vice versa. Experimentally, it is known that the magnetization of LSMO is out-of-plane for films with compressive strain and in-plane for films with tensile strain [15-17]. Interpreting these experiments in the Néel framework, it is concluded that $L^{\prime}<0$.

The elongated unit cell results in an out-of-plane easy axis, but the unit cell with a shortened out-of-plane lattice parameter results in an easy plane anisotropy if only the dipolar terms are taken into account. Of course, the resulting in-plane 
anisotropy will be effected by the quadrupolar term. The in-plane anisotropy energy is given by:

$$
E=-\frac{Q_{0}}{2} \sin ^{2} 2 \phi
$$

This describes weak biaxial in-plane anisotropy, which has also been found experimentally for LSMO grown on $(001)_{c}$ STO [18, 20, 27-31]. As the easy axes of the biaxial anisotropy are aligned with the $\langle 110\rangle_{c}$ crystal directions (except for references $[27,28])$, it follows that $Q_{0}>0$.

\section{Strained bond angles}

To understand the effect of a change in bond angle, a simple cubic unit cell, with the bond angle of the side face in the $x z$ plane equal to $\alpha=90^{\circ}-2 \delta$, is considered. The long face diagonal is aligned with $z$, as shown in figure 4.1b. All bond lengths are equal. The dominant term in the anisotropy energy in the $x z$ plane is given by:

$$
E=4 \delta L_{0}\left(\cos ^{2} \theta-\frac{1}{2}\right)
$$

The anisotropy energy in the $x y$ plane is:

$$
E=-2 \delta L_{0} \cos ^{2} \phi
$$

For values of $L_{0}<0$, an easy axis is present, aligned with the long body diagonal and in this case, the magnetization energy minimum in the $x y$ plane is in the $y$ direction. For the opposite case of $L_{0}>0$, the easy axis is aligned with the short body diagonal in the $x$ direction. This type of distortion is encountered in experiments on $(011)_{\mathrm{pc}}$ oriented thin films, where the strain not only changes the bond lengths but also the bond angles. The experimental data available on $(011)_{\mathrm{pc}}$ oriented thin films [28], however, is insufficient to determine the sign of $L_{0}$, as differently strained bond lengths are also present.

In bulk, LSMO has a rhombohedral crystal structure, in which the simple cubic structure is shortened along one $[111]_{p c}$ body diagonal. This results in changes in all the angles of the unit cell. For the calculations, the shortened body diagonal is aligned with the $z$ direction. The distortion is modelled with an angle $\delta>0$, such that the largest angle in each side face is $\alpha=90^{\circ}+2 \delta$. The dominant term in the anisotropy energy is:

$$
E=-6 \delta L_{0}\left(\cos ^{2} \theta-\frac{1}{2}\right),
$$

which results in an easy axis aligned with $z$, the short body diagonal for $L_{0}>0$ and an easy plane in the $x y$ plane for $L_{0}<0$. Experimentally both an easy axis aligned with the shortened body diagonal has been proposed [12] as well as an easy plane anisotropy $[13,14]$. Further measurements are therefore required to determine the sign of $L_{0}$, which will be discussed in the next section. 


\subsubsection{Summary model}

With the Néel model described in this section, the anisotropy of LSMO can be predicted if the crystal structure (lattice vectors of the Mn-Mn bonds) is known. For certain crystal structures, analytical results have been obtained which can directly be applied. For more complicated crystal structures, the anisotropy can be found by numerical calculations. The result depends on three phenomelogical parameters $L_{0}, L^{\prime}$ and $Q_{0}$.

The model can easily be extended with surface and stepedge anisotropy, by considering missing bonds and possibly modified bonds at the surface or stepedges. If the model is used to determine anisotropy energy as well as the easy and hard axes directions, it is important to include the surface anisotropy, which can significantly affect the results [32].

\subsection{Experiments}

In this section, the experimental results for LSMO grown on different surface plane orientations of NGO are presented. For four out of the five investigated orientations, the anisotropy is uniaxial and aligned with principal crystal directions. The anisotropy directions can be explained with the Néel model. The fifth orientation investigated, NGO $(112)_{\mathrm{o}}$, shows a change in anisotropy directions with film thickness.

\subsubsection{Crystal structure}

The orthorhombic crystal structure of NGO has lattice parameters, $\mathbf{a}=5.43 \AA$, $\mathbf{b}=5.50 \AA$ and $\mathbf{c}=7.71 \AA$ [33]. Because the NGO lattice parameters are all different, there are various in-plane strain states possible for the LSMO films, depending on the substrate surface plane orientation. The five different surface planes used here are shown in figure 4.2. The NGO $(110)_{\mathrm{o}}$ substrate orientation results in a $(001)_{\mathrm{pc}}$ oriented LSMO film, due to the cube-on-cube stacking. The in-plane sides of the LSMO pseudo-cube are aligned along the $[\overline{1} 10]_{\mathrm{o}}$ and the $[001]_{\mathrm{o}}$ lattice directions of the NGO substrate. The only other NGO orientation, that results in LSMO $(001)_{\mathrm{pc}}$ growth, is NGO (001) $)_{\mathrm{o}}$. In that case, the LSMO cube is rotated in-plane over $45^{\circ}$ with respect to the NGO $[100]_{\mathrm{o}}$ direction and the pseudocube is in-plane aligned along the $[110]_{\mathrm{o}}$ and $[1 \overline{1} 0]_{\mathrm{o}}$ lattice directions respectively. The two possible surface plane orientations for $(001)_{\mathrm{pc}}$ growth have different values for the lattice mismatch, but the most important difference is the symmetry of the surface unit cell. The angle between the $[\overline{1} 10]_{\mathrm{o}}$ and the $[001]_{\mathrm{o}}$ lattice directions in the NGO $(110)_{\mathrm{o}}$ surface unit cell is $90^{\circ}$, whereas the angle between the $[110]_{\mathrm{o}}$ and $[1 \overline{1} 0]_{\mathrm{o}}$ lattice directions in the NGO $(001)_{\mathrm{o}}$ surface unit cell is $89.3^{\circ}$. The NGO $(010)_{\mathrm{o}},(100)_{\mathrm{o}}$ and $(112)_{\mathrm{o}}$ substrates result in growth in the $(011)_{\mathrm{pc}}$ direction of the LSMO, with different values for the lattice mismatch along the two in-plane directions for each substrate orientation. For the NGO (100), and NGO (010)。 
a (110)。

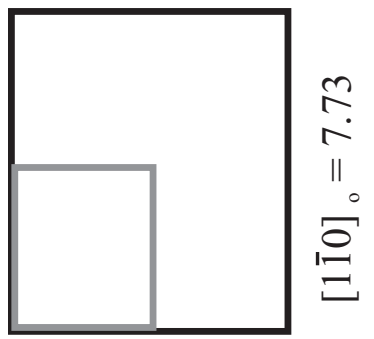

$[001]_{0}=7.71$ b (001)。

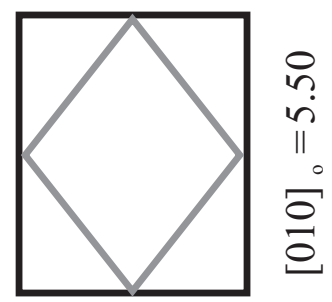

$[100]_{0}=5.43$
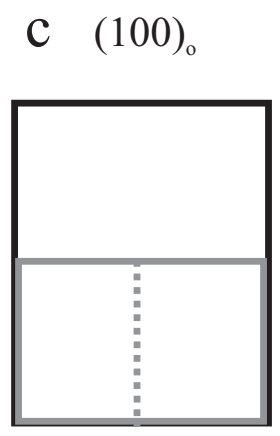

$[010]_{\mathrm{o}}=5.50$ d (010)

e $(112)_{0}$

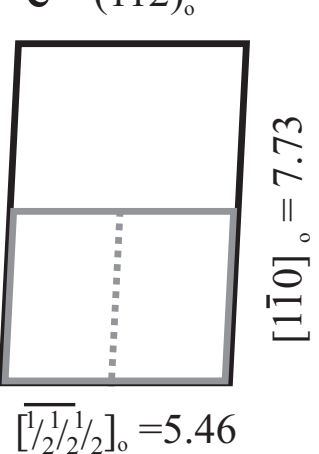

Figure 4.2: The surface planes of NGO. The pseudocubic surface unit cells are indicated in grey.

surfaces, the angle between the in-plane lattice directions is $90^{\circ}$ but for the NGO (112)o surface, the angle between the two lattice directions is $89.5^{\circ}$.

\subsubsection{Experimental results}

The LSMO thin films $(<50 \mathrm{~nm})$ were grown on the NGO substrates of different orientations $\left((110)_{\mathrm{o}},(001)_{\mathrm{o}},(100)_{\mathrm{O}},(010)_{\mathrm{o}}\right.$ and $\left.(112)_{\mathrm{o}}\right)$ by pulsed laser deposition. The surface treatments necessary for a singly terminated surface are described elsewhere [34]. The deposition was made with the use of a stoichiometric target in an oxygen background pressure of 0.35 mbar with a laser fluence of $3 \mathrm{~J} / \mathrm{cm}^{2}$ and at a substrate temperature of $750^{\circ} \mathrm{C}$. The target to substrate distance was fixed at $4 \mathrm{~cm}$. The films were cooled to room temperature at a rate of $10^{\circ} \mathrm{C} / \mathrm{min}$ in a 1 bar pure oxygen atmosphere. Atomic force microscopy measurements showed smooth surfaces with unit cell high steps. The step height was determined to be $\sim 3.9 \AA$ and $\sim 2.7 \AA$ for LSMO $(001)_{\mathrm{pc}}$ and LSMO $(011)_{\mathrm{pc}}$ respectively. 
X-ray diffraction (XRD) measurements were used to determine the directions of the crystal axes of the NGO substrates and the structure of the LSMO films. The length of the pseudocubic lattice vector in the out-of-plane direction can be obtained from XRD $\theta-2 \theta$ measurements. In comparison with the bulk LSMO value, an out-of-plane elongation of the unit cell is found for all surface plane orientations, indicative of the compressive strain in the films. In order to determine the length of the in-plane lattice vectors, reciprocal space mapping of an asymmetric reflection has to be performed, as discussed in section 3.4. For the determination of the angle between the two in-plane lattice vectors, which is important for films grown on NGO (001)o and NGO (112)o, reciprocal space maps of a set symmetry related peaks, with the in-plane momentum transfer aligned with the surface unit cell diagonals, were performed. As the angle between the lattice vectors results in a long and short diagonal, the amount of in-plane momentum transfer is not equal for the peaks in this set of reflections. With this difference, the angular distortion can be calculated. The lengths and angles of the pseudocubic unit cell of LSMO, grown on the different surface plane orientations of NGO, are summarized in table 4.1. The measurements show that all films are fully coherently strained to the substrate surface.

Vibrating Sample Magnetometer (VSM) magnetization measurements (Model10 VSM by Microsense) were used to determine the easy axis directions. Room temperature hysteresis loops were measured as a function of the in-plane field angle, $\Phi_{\mathrm{H}}$. The loops show the typical features of a uniaxial anisotropy: a square loop in the easy direction and an approximately linear $M-H$ dependence in the hard direction. The remanence versus field angle $\left(M_{\mathrm{r}}-\Phi_{\mathrm{H}}\right)$ curves of all samples show the expected behaviour of in-plane uniaxial anisotropy, described by $M_{\mathrm{r}}\left(\Phi_{\mathrm{H}}\right)=M_{\mathrm{r}}\left(\Phi_{\text {easy }}\right)\left|\cos \left(\Phi_{\mathrm{H}}-\Phi_{\text {easy }}\right)\right|$, where $\Phi_{\text {easy }}$ is the direction of (the in-plane component of ) the magnetic easy axis. Figure 4.3 shows hysteresis loops along both easy and hard directions and typical $M_{\mathrm{r}}-\Phi_{\mathrm{H}}$ curves of LSMO films on both NGO (100), and NGO (001)。. The magnetic easy and hard axes are found to be along in-plane NGO-crystal directions. For LSMO films on NGO (110) o and NGO $(010)_{\mathrm{o}}$, the easy and hard directions are aligned with the in-plane NGOcrystal directions as well. A summary of the easy and hard axis directions is given in Table 4.2. Any influence of the surface steps on the magnetic anisotropy directions, as was observed earlier for LSMO on STO $(001)_{c}$, was not observed. The experiments were repeated at low temperature $(150 \mathrm{~K})$ as well and no changes in the easy and hard directions were found.

The anisotropy of LSMO films grown on NGO (112)o changes with film thickness. Figure 4.4 shows the measured remanent magnetization $M_{\mathrm{r}}\left(\Phi_{\mathrm{H}}\right)$ of LSMO thin films with varying thicknesses. The films show clear signatures of uniaxial anisotropy with $180^{\circ}$ periodicity. With increasing thickness, the maxima in $M_{\mathrm{r}}$ shift from about $15^{\circ}$ with respect to the $[\overline{11} 1]_{\mathrm{o}}$ lattice direction to higher in-plane angles and the maxima decrease in height. Further, in the hard axis direction at $\phi_{\mathrm{H}}=105^{\circ}$ a discontinuity in the $M_{\mathrm{r}}-\Phi_{\mathrm{H}}$ curve is developing with increasing film thickness. The shift in the easy axis direction is seen more clearly in figure 4.4b where the magnetization direction at remanence, measured with a vector VSM, is plotted versus the angle $\Phi_{\mathrm{H}}$. The magnetic properties of LSMO films with thick- 
Table 4.1: Pseudocubic lattice parameters of LSMO, grown on NGO with different surface-plane orientations, as determined from XRD measurements. The in-plane lattice parameters are equal to those of the corresponding substrate lattice parameters. The error in the length is $0.005 \AA$ and the error in the angle is $0.1^{\circ}$.

\begin{tabular}{|c|c|c|c|}
\hline $\begin{array}{l}\text { LSMO pseudocube } \\
\text { lattice parameters }\end{array}$ & $\begin{array}{l}\text { Length } \\
(\AA)\end{array}$ & $\begin{array}{l}\text { LSMO pseudocube } \\
\text { angles }\end{array}$ & $\begin{array}{c}\text { Angle } \\
\left({ }^{\circ}\right)\end{array}$ \\
\hline \multicolumn{4}{|c|}{ LSMO $(001)_{\mathrm{pc}}$ on NGO $(110)_{\mathrm{o}}$} \\
\hline $\mathbf{a}=\mathbf{c}_{\mathrm{NGO}} / 2($ in-plane $)$ & 3.85 & $\alpha$ & 89.6 \\
\hline $\mathbf{b}=1 / 2 \sqrt{\mathbf{a}_{\mathrm{NGO}}^{2}+\mathbf{b}_{\mathrm{NGO}}^{2}}$ (in-plane) & 3.86 & $\beta$ & 90 \\
\hline c (out-of-plane) & 3.91 & $\gamma$ & 90 \\
\hline \multicolumn{4}{|c|}{$\operatorname{LSMO}(001)_{\mathrm{pc}}$ on NGO $(001)_{\mathrm{o}}$} \\
\hline $\mathbf{a}=1 / 2 \sqrt{\mathbf{a}_{\mathrm{NGO}}^{2}+\mathbf{b}_{\mathrm{NGO}}^{2}}($ in-plane $)$ & 3.86 & $\alpha$ & 90 \\
\hline $\mathbf{b}=1 / 2 \sqrt{\mathbf{a}_{\mathrm{NGO}}^{2}+\mathbf{b}_{\mathrm{NGO}}^{2}}$ (in-plane) & 3.86 & $\beta$ & 90 \\
\hline c (out-of-plane) & 3.91 & $\gamma$ & 89.3 \\
\hline \multicolumn{4}{|c|}{ LSMO $(011)_{\mathrm{pc}}$ on NGO $(100)_{\mathrm{o}}$} \\
\hline $\mathbf{a}=\mathbf{c}_{\mathrm{NGO}} / 2($ in-plane $)$ & 3.85 & $\alpha$ & 89.9 \\
\hline b & 3.89 & $\beta$ & 90 \\
\hline c & 3.89 & $\gamma$ & 90 \\
\hline$|(\mathbf{b}-\mathbf{c})|=\mathbf{b}_{\mathrm{NGO}}($ in-plane $)$ & 5.50 & $\angle[\mathbf{a},(\mathbf{b}-\mathbf{c})]$ & 90 \\
\hline \multirow[t]{2}{*}{$|(\mathbf{b}+\mathbf{c})|$ (out-of-plane) } & 5.51 & $\angle[\mathbf{a},(\mathbf{b}+\mathbf{c})]$ & 90 \\
\hline & & $\angle[(\mathbf{b}-\mathbf{c}),(\mathbf{b}+\mathbf{c})]$ & 90 \\
\hline \multicolumn{4}{|c|}{ LSMO $(011)_{\mathrm{pc}}$ on NGO $(010)_{\mathrm{o}}$} \\
\hline $\mathbf{a}=\mathbf{c}_{\mathrm{NGO}} / 2$ (in-plane) & 3.85 & $\alpha$ & 88.7 \\
\hline b & 3.88 & $\beta$ & 90 \\
\hline c & 3.88 & $\gamma$ & 90 \\
\hline$|(\mathbf{b}-\mathbf{c})|=\mathbf{a}_{\mathrm{NGO}}($ in-plane $)$ & 5.43 & $\angle[\mathbf{a},(\mathbf{b}-\mathbf{c})]$ & 90 \\
\hline \multirow[t]{2}{*}{$|(\mathbf{b}+\mathbf{c})|$ (out-of-plane) } & 5.55 & $\angle[\mathbf{a},(\mathbf{b}+\mathbf{c})]$ & 90 \\
\hline & & $\angle[(\mathbf{b}-\mathbf{c}),(\mathbf{b}+\mathbf{c})]$ & 90 \\
\hline \multicolumn{4}{|c|}{ LSMO $(011)_{\mathrm{pc}}$ on NGO $(112)_{\mathrm{o}}$} \\
\hline $\mathbf{a}=1 / 2 \sqrt{\mathbf{a}_{\mathrm{NGO}}^{2}+\mathbf{b}_{\mathrm{NGO}}^{2}}$ (in-plane) & 3.86 & $\alpha$ & 89.4 \\
\hline b & 3.88 & $\beta$ & 89.6 \\
\hline c & 3.88 & $\gamma$ & 89.6 \\
\hline \multirow{3}{*}{$\begin{array}{l}|(\mathbf{b}-\mathbf{c})|=1 / 2 \sqrt{\mathbf{a}_{\mathrm{NGO}}^{2}+\mathbf{b}_{\mathrm{NGO}}^{2}+\mathbf{c}_{\mathrm{NGO}}^{2}} \\
(\text { in-plane }) \\
|(\mathbf{b}+\mathbf{c})| \text { (out-of-plane) }\end{array}$} & 5.46 & $\angle[\mathbf{a},(\mathbf{b}-\mathbf{c})]$ & 89.5 \\
\hline & 5.52 & $\angle[\mathbf{a},(\mathbf{b}+\mathbf{c})]$ & 90 \\
\hline & & $\angle[(\mathbf{b}-\mathbf{c}),(\mathbf{b}+\mathbf{c})]$ & 90 \\
\hline
\end{tabular}



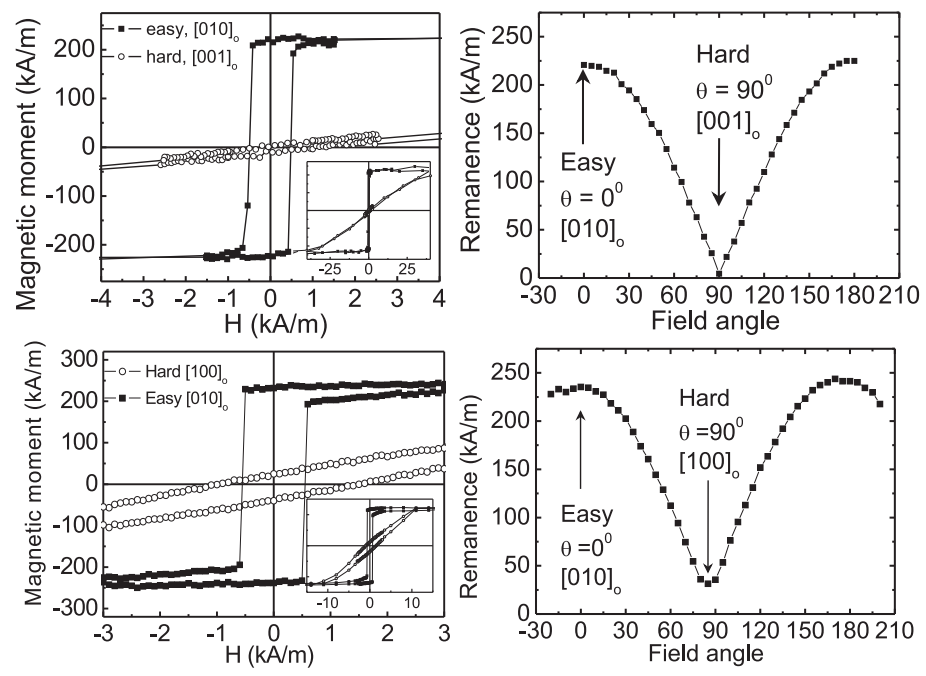

Figure 4.3: Top panel: Left: Hysteresis loops of a $14 \mathrm{~nm}$ thick LSMO/NGO (100)o film along in-plane easy and hard directions. Right: Remanence vs in-plane field angle at room temperature. Arrows denote easy and hard directions. Bottom panel: Left: Hysteresis loops of a $25 \mathrm{~nm}$ thick LSMO film grown on NGO (001)o, along in-plane easy and hard directions. Right: Remanence vs in-plane field angle at room temperature. Arrows denote easy and hard directions. This figure is published identically in [36].

nesses greater than $150 \mathrm{~nm}$ show additional maxima and minima in the $M_{\mathrm{r}}-\Phi_{\mathrm{H}}$ curves. This is due to a partial relaxation of the LSMO crystal structure and is discussed in more detail elsewhere [35].

\subsubsection{Analysis of the experiments in terms of the Néel mo- del}

The magnetic anisotropy, found for LSMO grown on the NGO surface orientations discussed above, can be modelled with the Néel model. It is assumed that the quadrupolar terms are too small to be of influence. Only the in-plane anisotropy energy is calculated, as this can be directly compared with the experiments. In the experiments, the magnetization is predominantly in-plane due to the effect of the demagnetization of a thin film. For LSMO grown on NGO (001) $)_{\mathrm{o}}$, the two in-plane bonds are of equal length and the anisotropy energy is given by equation 4.5 :

$$
E=4 \delta L_{0} \cos ^{2} \Phi_{\mathrm{M}}
$$

where $\delta=0.0065 \mathrm{rad} . \Phi_{\mathrm{M}}=0$ corresponds to the long surface unit cell diagonal. This direction is aligned with the NGO $[010]_{\mathrm{o}}$ direction and is the easy axis. 

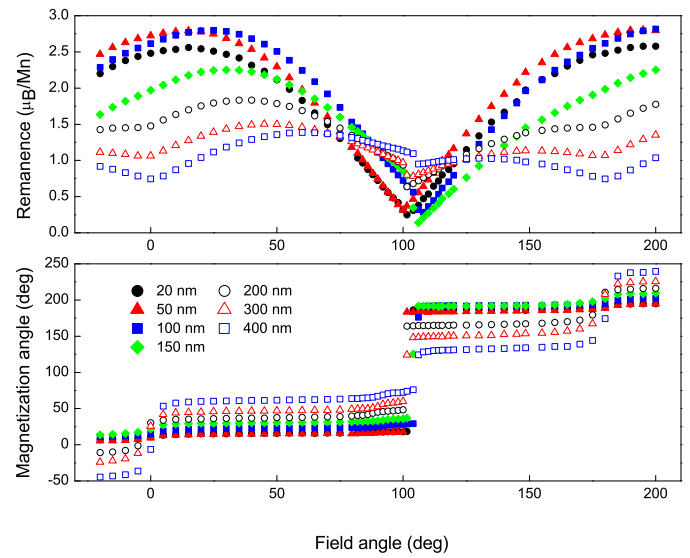

Figure 4.4: Field angle $\Phi_{\mathrm{H}}$ dependence of (a) the in-plane remanent magnetization $M_{\mathrm{r}}$ and (b) the magnetization angle $\Phi_{\mathrm{M}}$. $\Phi_{\mathrm{H}}=0^{\circ}$ corresponds to the $[\overline{11} 1]_{\mathrm{o}}$ crystal direction, $\Phi_{\mathrm{H}}=89.5^{\circ}$ to the $[\overline{1} 10]_{\mathrm{o}}$ direction and $\Phi_{\mathrm{H}}=180^{\circ}$ to the $[11 \overline{1}]_{\mathrm{o}}$ direction.

Table 4.2: Magnetic easy and hard axis for LSMO grown on NGO. The model easy and hard directions have been calculated using the Néel model.

\begin{tabular}{lccc}
\hline \hline & In-plane direction & Experiment & Model \\
\hline \multirow{2}{*}{ LSMO $(001)_{\mathrm{pc}}$ on NGO $(110)_{\mathrm{o}}$} & {$[1 \overline{10}]_{\mathrm{o}}$} & Easy & Easy \\
& {$[001]_{\mathrm{o}}$} & Hard & Hard \\
LSMO $(001)_{\mathrm{pc}}$ on NGO $(001)_{\mathrm{o}}$ & {$[100]_{\mathrm{o}}$} & Hard & Hard \\
& {$[010]_{\mathrm{o}}$} & Easy & Easy \\
LSMO $(011)_{\mathrm{pc}}$ on NGO $(100)_{\mathrm{o}}$ & {$[010]_{\mathrm{o}}$} & Easy & Easy \\
& {$[001]_{\mathrm{o}}$} & Hard & Hard \\
LSMO $(011)_{\mathrm{pc}}$ on NGO $(010)_{\mathrm{o}}$ & {$[100]_{\mathrm{o}}$} & Hard & Hard \\
& {$[001]_{\mathrm{o}}$} & Easy & Easy \\
\hline \hline
\end{tabular}


Therefore, it is concluded that $L_{0}<0$. This implies as well that bulk LSMO is expected to have an easy plane anisotropy and not an easy axis anisotropy.

For LSMO grown on NGO (110), the two in-plane bonds are perpendicular to each other and the bonds are strained by an unequal amount. The anisotropy energy is given by equation 4.3 :

$$
E=\Delta \epsilon L^{\prime} \cos ^{2} \Phi_{\mathrm{M}}
$$

where $\Delta \epsilon=0.0025$ is the difference in strain between the two in-plane directions and $\Phi_{\mathrm{M}}=0$ corresponds to the longest bond, which is aligned with the NGO $[1 \overline{1} 0]_{\mathrm{o}}$ lattice direction (easy axis). In this case, the third LSMO bond is not perpendicularly oriented to the surface plain and will effect the in-plane anisotropy

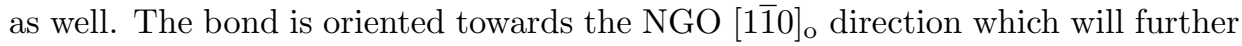
minimize the anisotropy energy in this direction. However, this effect is much smaller compared to the effect of the difference in strain, within the acceptable magnitudes of $L_{0}$ and $L^{\prime}$.

For LSMO grown on NGO (100), and NGO (010), two mechanisms contribute to the in-plane anisotropy. The angle distortion between the $\mathbf{b}$ and $\mathbf{c}$ vectors minimizes the energy for magnetization aligned with the a vector (parallel to the

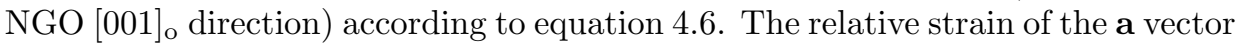
compared with $\mathbf{b}$ and $\mathbf{c}$ minimizes the energy for magnetization aligned with the b $\overline{\mathbf{c}}$ vector (NGO $[010]_{\mathrm{o}}$ respectively $[100]_{\mathrm{o}}$ direction) according to equation 4.3. The total energy is given by:

$$
E=2 \delta L_{0} \cos ^{2} \Phi_{\mathrm{M}}-\Delta \epsilon L^{\prime} \cos ^{2} \Phi_{\mathrm{M}}
$$

where $\delta=\pi(90-\alpha) / 360, \Delta \epsilon=(|b|-|a|) / 3.88$ and $\Phi_{\mathrm{M}}=0$ corresponds to the a direction $\left(\mathrm{NGO}[001]_{\mathrm{o}}\right)$. For LSMO on NGO $(100)_{\mathrm{o}}$ the second term is much larger than the first for typical values of $L_{0}$ and $L^{\prime}$ and the easy axis is in the $[010]_{\mathrm{o}}$ direction. In the case of LSMO on NGO $(010)_{\mathrm{o}}$, both an easy direction in the $(100)_{\mathrm{o}}$ and $(001)_{\mathrm{o}}$ can be found, depending on the value of the $L^{\prime} / L_{0}$ ratio. In order to match the experimental results with the model, the ratio has to be smaller than 2.6. With this constraint on the parameters, $0<L^{\prime} / L_{0} \leq 2.6$, the model reproduces the experimentally found anisotropy.

For the LSMO on NGO (112)o, a simple analytical expression for the anisotropy energy does not exist. The anisotropy energy was numerically calculated with the inner products of the magnetization vector and the three unit cell vectors, as a function of the in-plane angle $\Phi_{M} . \Phi_{M}$ is defined with respect to the in-plane lattice vectors in the same way as $\Phi_{\mathrm{H}}$ in the caption of figure 4.4. There are two main contributions to the anisotropy energy. The angle between the in-plane lattice directions of $89.5^{\circ}$ results in a contribution to the anisotropy energy with a minimum when the magnetization is at $\Phi_{M}=62^{\circ}$. The other contribution to the anisotropy energy is the strain difference between the partially out-of-plane unit cell vectors and the in-plane unit cell vector. This gives a minimum in energy when $\Phi_{\mathrm{M}}=0^{\circ}$. Depending on the $L^{\prime} / L_{0}$ ratio, intermediate angles are found as the easy axis direction. 


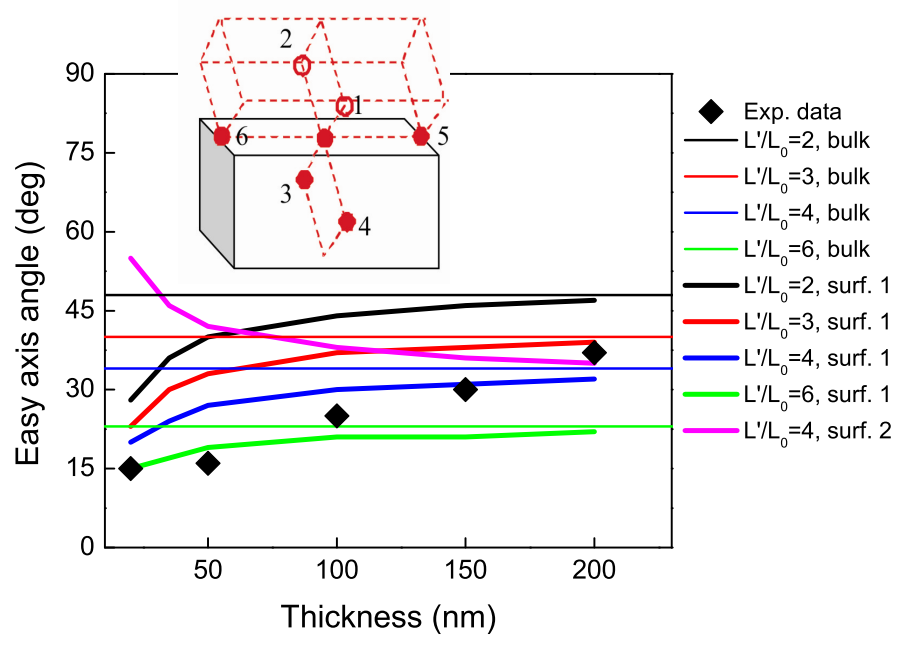

Figure 4.5: In-plane easy axis direction as function of film thickness for LSMO on NGO (112)o. The curves with increasing easy axis angle with increasing thickness are calculated assuming bulk and surface anisotropy (surf. 1) with no interaction of the surface in-plane bonds (Mn ions 5 and 6 of the schematic in the inset) and for different Néel parameter ratios. The curve with decreasing easy axis angle with increasing thickness is calculated assuming full interaction with Mn5 and Mn6 (surf. 2). The horizontal lines are for bulk anisotropy only. The inset shows a schematic of the LSMO crystal structure with the surface atoms indicated.

In order to explain the difference in anisotropy with increasing film thickness, a surface anisotropy term is added. The magnitude of the surface anisotropy scales with the surface to volume ratio of the LSMO. A sketch of the atoms in the surface unit cell is given in figure 4.5. Atoms 1 and 2 are missing, atoms 5 and 6 are at the surface, which might modify the interaction, and atoms 3 and 4 are considered bulk like. When only the missing atoms are taken into account, the surface anisotropy energy contribution gives a minimum at $\Phi_{\mathrm{M}}=89.5^{\circ}$. When the interaction of atoms 5 and 6 is removed as well, the surface anisotropy has a minimum at $\Phi_{\mathrm{M}}=0^{\circ}$. The latter configuration results in an increase of the easy axis angle with film thickness, as observed in the experiments. In figure 4.5 the experimentally found angles for the easy axes are compared with the model results. The Néel model can describe the trend in the anisotropy, although it should be noted that the model predicts an anisotropy energy where the easy and hard axes directions are orthogonal. This predicts a hard axis which shifts with film thickness as well, while in the experiments it was found that the hard axis is fixed at $\Phi_{\mathrm{H}}=105^{\circ}$. 


\subsubsection{Summary experiments}

The experiments uniquely determine the sign of the two anisotropy parameters, $L_{0}$ and $L^{\prime}$, which are both negative. This corresponds well with the earlier reported observation that the magnetization is predominantly out-of-plane for films under compressive strain and in-plane for films under tensile strain. Moreover, the negative sign of $L_{0}$ implies that bulk LSMO should have an easy plane anisotropy, which is in agreement with the conclusions from references $[13,14]$, but not with reference [12]. It is suggested that the latter experiment can also be interpreted with an easy plane anisotropy, together with further symmetry breaking due to the presence of the surface.

The fact that both parameters are negative implies that the interaction strength in the Néel model increases with increasing distance. This is contrary to the expectation which would suggest a decrease of the interaction strength with increasing bond length. This is discussed in more detail in the next section about the microscopic origin of the anisotropy.

\subsection{Microscopic origin of the anisotropy}

The origin of magnetocrystalline anisotropy is a combination of spin orbit coupling and crystal field interaction [23]. For insulating materials, it is well understood in the single ion anisotropy model [23]. For example, in the case of the LSMO parent compound $\mathrm{LaMnO}_{3}$ (LMO), each oxygen octahedron is distorted by the Jahn Teller distortion. This results in an occupied low energy $\mathrm{d}_{3 \mathrm{x}^{2}-\mathrm{r}^{2}}$ orbital and this orbital has a small orbital angular momentum in the $x$ direction. For this ion, it is energetically favourable for the magnetization to be aligned with the $x$ direction. The groundstate of LMO has $d_{3 x^{2}-r^{2}} d_{3 y^{2}-r^{2}}$ orbital ordering in the ab plane. This results in an averaging of the anisotropy energy within the ab plane and the $\mathbf{c}$ axis becomes the hard axis. Finally, the orthorhombic crystal structure (with $\mathbf{b}=x y>\mathbf{a}=x \bar{y}$ ) rotates all Jahn Teller distorted octahedra towards the $\mathbf{b}$ direction, resulting in the $\mathbf{b}$ axis as the magnetic easy axis [24, 37].

For conducting materials, two approaches exist [23]. The first is to consider electrons which are temporarily trapped at a lattice site as the main source of anisotropy [38]. These electrons can then be treated identically to the electrons in an insulating compound within the framework of single ion anisotropy. The other approach would be to solve the electron wavefunction of the conducting compound and directly use this to calculate the orbital angular momentum and the spin orbit coupling.

In LSMO, both mobile electrons, the $e_{g}$ band, as well as immobile electrons, the core spins in the $t_{2 \mathrm{~g}}$ band, are present. As the majority of the electrons is not mobile, the anisotropy is analyzed within the framework of the single ion model. Moreover, a large part of the $\mathrm{e}_{g}$ electrons is trapped and does not contribute to the conductivity [39]. The analysis involves a discussion about possible oxygen octahedra rotations and deformations as a response to the applied strain. It will 
be shown that the main result of this chapter, the negative sign of both $L_{0}$ and $L^{\prime}$, can only be reproduced within this model, when an oxygen octahedra bond angle distortion is taken into account.

\subsubsection{Single ion model}

In the single ion model of anisotropy [23], the magnetic ion is assumed to have strong spin orbit coupling and the magnetocrystalline anisotropy is due to the electrostatic interaction of the atomic orbital with the surrounding crystal field. The crystal field is described with a point charge model, where each nearest neighbour ligand can be evaluated separately. The anisotropy energy contribution of a single ligand is given by:

$$
E_{i}=-\frac{e Q Q_{2}}{16 \pi \epsilon_{0} R_{i}^{3}}\left(3 \cos ^{2} \theta_{i}-1\right),
$$

in which $e$ is the electron charge, $Q$ is the effective charge of the ligand, $Q_{2}$ describes the orbital of the magnetic ion in a multipole expansion, $\epsilon_{0}$ is the permittivity of free space, $R_{i}$ is the distance from the magnetic ion to the ligand and $\theta_{i}$ is the angle between the magnetization (as well as the direction of the orbital due to the strong spin orbit coupling) and the ligand bond vector. The parameter $Q_{2}$ describes the shape of the charge distribution which is oblate (pancake like) for positive $Q_{2}$ and prolate (cigar like) for negative $Q_{2}$. As almost all ligands have an effective negative charge, magnetization in the ligand direction is favoured for oblate orbitals. In order to calculate the anisotropy, a summation over the ligands of the magnetic ion and all the magnetic ions in the unit cell has to be made (only nearest neighbour interactions are taken into account). For LSMO, this amounts to the $3 \mathrm{O}$ ions, which describe the octahedron of a single $\mathrm{Mn}$ ion, and effectively 3 pairs of $\mathrm{Mn}$ ions, to include the effect of octahedra rotations correctly.

\section{Anisotropy energy of a spin pair}

In figure 4.6, a schematic of a pair of $\mathrm{Mn}$ ions is presented together with their oxygen octahedra. The plane of the Mn-O-Mn bond lies perpendicular to the direction of view, i.e. in the plane of the paper. One octahedron is rotated clockwise by an amount of $\alpha$ and the other one anti-clockwise by the same amount. The angle $\beta$ is used to describe octahedron bond angle distortion and the angles $\zeta$ and $\omega$ define the direction of the magnetization, as shown in the figure. In the Néel model, the dipolar term describing the anisotropy between two Mn ions Mn1 and Mn2 is given by:

$$
E_{12}=L\left(r_{12}\right) \cos ^{2} \zeta .
$$

In the single ion model, the energy $E_{12}$ is a sum of two Mn-O interactions, shown in figure 4.6:

$$
E_{12}=\sum_{i=1,2}-\frac{3 e Q Q_{2}}{16 \pi \epsilon_{0} R_{i}^{3}} \cos ^{2} \theta_{i},
$$




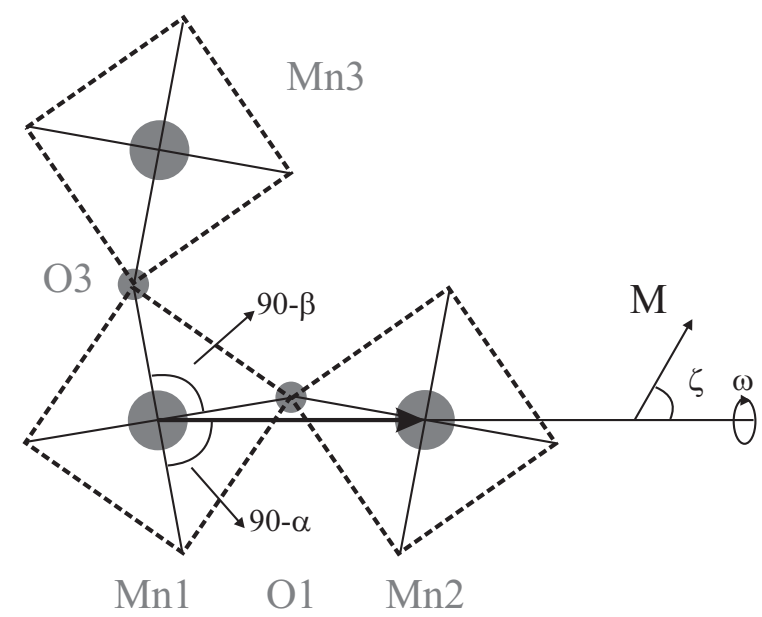

Figure 4.6: The rotation of the oxygen octahedra and the position of the oxygen ligands with respect to the Mn-Mn bond vector. The Mn-Mn bond vector is indicated with the thick black arrow. The plane of the Mn-O$\mathrm{Mn}$ bond lies perpendicular to the direction of view, i.e. in the plane of the paper. The direction of the magnetization is defined using the angles $\zeta$ (angle between the magnetization $\mathrm{Mn}-\mathrm{Mn}$ bond vector) and $\omega$ (angle between the magnetization and the Mn-O-Mn bond plane, defined such that $\omega=0$ corresponds to magnetization within this plane.) The angle $\alpha$ describes the rotation of the two $\mathrm{MnO}_{6}$ octahedra, while the angle $\beta$ indicates the bond angle distortion of the octahedra.

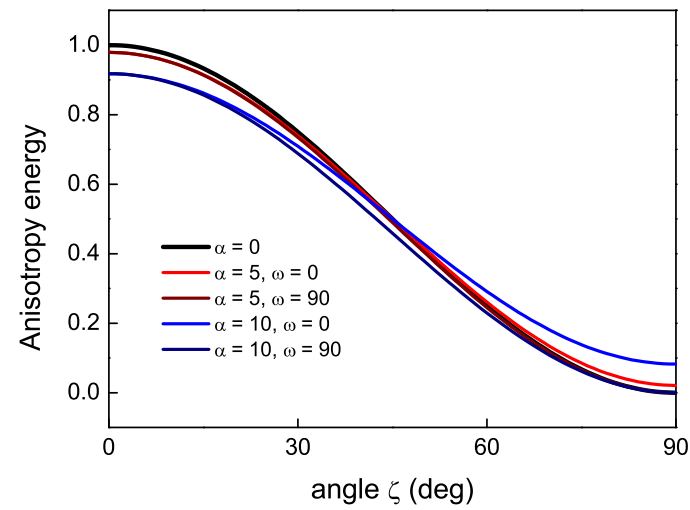

Figure 4.7: The angle dependence of the anisotropy energy for different values of the angles $\alpha$ and $\omega$, as calculated with equation 4.14. 
where the summation describes the effect of the centre oxygen on the Mn1 and Mn2. This equation can be rewritten in terms of $\zeta, \alpha, \beta$ and $\omega$ :

$$
E_{12}=-\frac{3 e Q Q_{2}}{8 \pi \epsilon_{0} R_{M n O}^{3}}\left(\cos ^{2}(\alpha+\beta) \cos ^{2} \zeta+\sin ^{2}(\alpha+\beta) \sin ^{2} \zeta \cos ^{2} \omega\right) .
$$

Here, the first term is the anisotropy energy of the two ligands projected onto the Mn-Mn bond vector, while the second term is the projection perpendicular to the Mn-Mn bond vector. The oxygen ion is equidistant to the two $\mathrm{Mn}$ ions, so $R_{1}=R_{2}=R_{M n O}$. The $\zeta$ angle dependence of the anisotropy energy is plotted in figure 4.7 for some values of the angles $\alpha$ and $\omega$. An increase in $\alpha$ results in a decrease of the anisotropy energy around $\zeta=0$ and, for $\omega=0$, the increase of $\alpha$ also results in an increase of the anisotropy energy around $\zeta=90$. The relation between the distance between the $\mathrm{Mn}$ ions and the distance between the $\mathrm{Mn}$ and the $\mathrm{O}$ ion is:

$$
R_{M n O}=\frac{r_{12}}{2 \cos (\alpha+\beta)} .
$$

\subsubsection{Oxygen octahedra rotation and deformation}

The anisotropy energy in the single ion model has, in contrast to the Néel model, an explicit bond length dependence. Therefore, the effect of strain can, in principle, be directly calculated by derivation with respect to $R_{i}$ in equation 4.11 . For the case of tensile strain, i.e. increasing $R_{i}$, the anisotropy energy decreases. This decrease is in contrast to the experimental results, discussed in the previous section, where an increase of the anisotropy energy with tensile strain was determined. However, the strain is applied to the LSMO Mn-Mn bonds and this does not automatically imply a change in the Mn-O bond length. Oxygen octahedra rotations and deformations can play a role as well. In the following, the anisotropy energy of a pair of Mn ions will be evaluated as a function of the strain applied to the Mn-Mn bond. Three approaches for the structural response to the applied strain, namely octahedra rotations, $\mathrm{Mn}-\mathrm{O}$ bond length variation and octahedron bond angle variation, are made and used to calculate the ratio between the Néel parameters $L^{\prime} / L_{0}$. It will be shown that only the octahedron bond angle variation is consistent with the experimentally observed anisotropy.

\section{Octahedra rotations}

The expected structure response of a perovskite oxide to applied strain is rotation of the oxygen octahedra. For strained $\mathrm{La}_{1-x} \mathrm{Ca}_{x} \mathrm{MnO}_{3}$ thin films, for example, it has been shown that the strain resulted in oxygen octahedra rotations [40]. Octahedra rotations are therefore expected for LSMO as well when strain is applied the $\mathrm{Mn}-\mathrm{Mn}$ bond. However, the rotations do not result in a change in the anisotropy energy, as can be directly understood from the following argument. For a perfect $\mathrm{MnO}_{6}$ octahedron, i.e. the angles between the three $\mathrm{Mn}-\mathrm{O}$ lattice vectors are 90 degrees and the $\mathrm{Mn}-\mathrm{O}$ distances are all equal, the anisotropy energy does not 
depend on the angle of the magnetization and therefore no anisotropy is present. The same holds for a crystal structure in which the octahedra are rotated but not distorted. For example, starting with a cubic perovskite unit cell, a tetragonal unit cell with $\mathbf{c}>\mathbf{a}$ results if the octahedra are rotated around the $\mathbf{c}$ axis. This structure does not have any anisotropy, as the octahedra are not distorted. This is in contrast to the Néel model, where either an [001] easy axis or an (001) easy plane is expected.

In equation 4.14, the anisotropy energy of the spin pair does depend on the amount of rotation $\alpha$. An increase in $\alpha$ reduces the anisotropy energy of the spin pair with a factor $1-\cos ^{2} \alpha$. However, the rotation $\alpha$ also changes the anisotropy energy of the other two spin pairs in the octahedron and they have a perpendicular anisotropy energy component. E.g. in figure 4.6, the anisotropy energy of the Mn1-Mn3 spin pair has a component in the Mn1-Mn2 direction as well, due to the octahedra rotation. This component is equal to $\sin ^{2} \alpha$ and it exactly balances the reduction of $1-\cos ^{2} \alpha$. So, this calculation also shows that a rotation of the oxygen octahedra does not change the anisotropy energy. Therefore, the strain dependence of the anisotropy energy cannot be explained by oxygen octahedra rotations. For anisotropy, octahedra distortions have to be included in the model. This can either be a bond length variation or a bond angle variation.

\section{Mn-O bond length variation}

Secondly, an Mn-O bond length variation proportional to the strain is considered. This bond length variation is very similar to the Jahn Teller distortion of the octahedra in the parent compound LMO. The physical picture is that unequal strain in different lattice directions results in an elongation of the oxygen octahedra in the direction $x$ with the most tensile strain. This results in preferential occupation of the $\mathrm{d}_{3 x^{2}-r^{2}}$ orbitals and the spin orbit coupling of these orbitals, with the easy axis in the $x$ direction, then explains the anisotropy.

The strain dependence of the anisotropy energy is calculated from equations 4.14 and 4.15. $\alpha$ and $\beta$ are constant, so:

$$
\mathrm{d} r_{12}=2 \cos (\alpha+\beta) \mathrm{d} R_{M n O} .
$$

The derivative of the anisotropy energy, equation 4.14, with respect to the distance $\mathrm{r}_{12}$ is then given by:

$$
\frac{\mathrm{d} E_{12}}{\mathrm{~d} r_{12}}=+\frac{9 e Q Q_{2}}{8 \pi \epsilon_{0} R_{M n O}^{4}}\left(\cos ^{2}(\alpha+\beta) \cos ^{2} \zeta+\sin ^{2}(\alpha+\beta) \sin ^{2} \zeta \cos ^{2} \omega\right) \frac{1}{2 \cos (\alpha+\beta)} .
$$

As the Néel parameter $L^{\prime}$ is defined as $L^{\prime}=\mathrm{d} E_{i j}\left(r_{0}\right) / \mathrm{d} r_{i j} \cdot r_{0}$, it follows that the ratio between the Néel parameters $L^{\prime} / L_{0}$ is equal to -3 . From the experiments in this chapter, it was concluded that the ratio has to be positive. Therefore, an $\mathrm{Mn}-\mathrm{O}$ bond length variation cannot explain the strain dependence of the magnetic anisotropy. Only the crystal distortions which are described by the Néel parameter 
$L^{\prime}$, bond length changes of the Mn-Mn lattice vectors, are explained and not the crystal distortions described by the Néel parameter $L_{0}$, bond angle changes between the Mn-Mn lattice vectors.

Note that a more complicated structure of Jahn Teller distorted octahedra, together with orbital ordering, similar to the magnetic anisotropy of LMO, can be applied to the cases where only one of the angles between the Mn-Mn lattice vectors is distorted, such as LSMO on NGO $(001)_{\mathrm{o}}$, but this cannot be applied in general. For example, for the bulk rhombohedral crystal structure, where all three angles between the Mn-Mn lattice vectors are distorted, it is not possible to have an orbitally ordered state without significant anisotropy, due to the Jahn Teller distorted octahedra, resulting in an easy axis in either the $[001]_{\mathrm{pc}},[010]_{\mathrm{pc}}$, or $[100]_{\mathrm{pc}}$ lattice direction instead of the $(111)_{\mathrm{pc}}$ plane. Also, it is known that the Jahn Teller distortion is severely reduced when cooling below $\mathrm{T}_{\mathrm{c}}[11]$. So, it is concluded that the magnetocrystalline anisotropy of LSMO is not due to Jahn Teller distortions of the octahedra.

\section{Bond angle variation}

Finally, a bond angle variation of the oxygen octahedra is considered. This is shown in figure 4.6 with the angle $\beta$, the angle between the Mn1-O1 vector and the plane spanned by the Mn1-O2 (perpendicular to the page) and the Mn1-O3 vectors. It is assumed that oxygen octahedra rotations are present such that $\beta<\alpha$ and that the axes of the rotations described by $\alpha$ and $\beta$ coincide. In this case, $\beta$ can be both negative and positive which describes tensile and compressive strain applied to the Mn-Mn bond vector respectively.

The strain dependence of the anisotropy energy is calculated from equations 4.14 and 4.15. $\alpha$ and $R_{M n O}$ are constant, so:

$$
\mathrm{d} r_{12}=-2 R_{M n O} \sin (\alpha+\beta) \mathrm{d} \beta .
$$

The derivative of the anisotropy energy, equation 4.14, with respect to the distance $\mathrm{r}_{12}$ is then given by:

$$
\frac{\mathrm{d} E_{12}}{\mathrm{~d} r_{12}}=-\frac{3 e Q Q_{2}}{8 \pi \epsilon_{0} R_{M n O}^{4}} \cos (\alpha+\beta)\left(\cos ^{2} \zeta-\sin ^{2} \zeta \cos ^{2} \omega\right)
$$

The ratio between the Néel parameters cannot be directly calculated, as it is a function of the angles $\zeta$ and $\omega$. Therefore, an effective anisotropy energy for the spin pair is defined as:

$$
E_{e f f}=E_{12}(\zeta=0)-E_{12}(\zeta=90)=-\frac{3 e Q Q_{2}}{8 \pi \epsilon_{0} R_{M n O}^{3}}\left(\cos ^{2}(\alpha+\beta)-\frac{1}{2} \sin ^{2}(\alpha+\beta)\right),
$$

in which the $\omega$ dependence of the perpendicular anisotropy has been averaged out, resulting in a factor $\frac{1}{2}$. This assumes that the orientations of the Mn-O-Mn planes 
for all the Mn-Mn bonds in the $r_{12}$ direction are randomly distributed. $E_{\text {eff }}$ does not depend on $\zeta$ and it can be used to calculate the amount of change in the anisotropy energy with strain. The ratio between the Néel parameters is given by

$$
L^{\prime} / L_{0}=\frac{6}{3-\frac{1}{\cos ^{2}(\alpha+\beta)}} .
$$

Around the equilibrium bond length, $\alpha=6.5^{\circ}[41]$ and $\beta=0^{\circ}$, the ratio is close to 3 .

\subsubsection{Implications for LSMO}

From the comparison of the experimental data and the Néel model, the signs of the dipolar Néel parameters are known. Both $L_{0}$ and $L^{\prime}$ are negative. From the analysis in terms of the single ion model, the sign of $L_{0}$ directly determines the sign of the orbital shape parameter $Q_{2}$. So, the basic shape of the orbital at the $\mathrm{Mn}$ site is oblate (pancake like). This is in contrast to the anisotropy of the parent compound $\mathrm{LaMnO}_{3}$ (LMO). In LMO the Jahn Teller distortion results in $e_{g}$ orbitals with a prolate character. Due to the itineracy of the $\mathrm{e}_{g}$ electrons in LSMO, it is suggested here that the main anisotropy is due to the $t_{2 g}$ electrons which are stationary. It is concluded that the $\mathrm{t}_{2 g}$ orbitals have an oblate character.

As it is also found that $L^{\prime}$ is negative, it can be expected from the single ion model that the octahedron bond angle changes with strain and the Mn-O bond length is relatively constant. The change in octahedron bond angle results in a ratio between the Néel parameters equal to 3. In this derivation, it was assumed that $\alpha$ does not change with strain. In practice, the strain will change both the amount of octahedra rotations $\alpha$, which does not induce anisotropy, and the amount of octahedron angle deformation $\beta$. Therefore, the obtained ratio between the Néel parameters is an upper limit and $0<L^{\prime} / L_{0} \leq 3$. This is in reasonable agreement with the Néel ratios used to explain the experiments, $0<L^{\prime} / L_{0} \leq 2.6$.

\subsection{Conclusions}

A model, relating the crystal structure to the magnetization, was developed to describe the magnetocrystalline anisotropy of LSMO. The model is based on the framework of Néel. Surface and stepedge induced anisotropy can easily be included in the calculations. The model can be used to predict the magnetic anisotropy for other LSMO/substrate combinations and LSMO subject to strain from e.g. piezoelectric materials.

The model predictions were compared to experimental data of LSMO films grown on different surface orientations of NGO crystals. The orthorhombic symmetry of NGO allowed for a number of surfaces with small differences in the surface unit cell symmetry. Most importantly, the $(001)_{\mathrm{o}}$ and (110), surface result in an LSMO crystal structure which is directly comparable with the basic structural distortions 
in the model. From the comparison between experiment and model predictions, the signs of the Néel parameters $L_{0}$ and $L^{\prime}$ are derived. These parameters are both negative, which implies that bulk LSMO has easy plane anisotropy. For LSMO grown on NGO (112) o, the anisotropy was found to change with film thickness. By including surface anisotropy in the model, a first step towards the understanding of the anisotropy could be taken.

The experiments uniquely demonstrate that both the Néel parameters $L_{0}$ and $L^{\prime}$ are negative. The origin of the anisotropy is the alignment of the Mn orbitals in the LSMO crystal field. The physical meaning of a negative $L_{0}$ is that the shape of the orbitals is oblate. This is in contrast to the parent compound LMO which has prolate orbitals. It is suggested that the main contribution to the anisotropy in LMO is due to prolate $\mathrm{e}_{g}$ orbitals, while the main contribution to the anisotropy of LSMO is due to oblate $t_{2 g}$ orbitals. The crystal field due to the neighbouring oxygen ions was evaluated with respect to strain. As $L^{\prime}$ was found to be negative as well, the analysis of the crystal field interaction suggests that the angles of the $\mathrm{MnO}_{6}$ octahedra change with strain, while the $\mathrm{Mn}-\mathrm{O}$ bond lengths remain approximately constant. 


\subsection{References}

[1] K. Choi, M. Biegalski, Y. Li, A. Sharan, J. Schubert, R. Uecker, P. Reiche, Y. Chen, X. Pan, V. Gopalan, L. Chen, D. Schlom, and C. Eom, "Enhancement of ferroelectricity in strained $\mathrm{BaTiO}_{3}$ thin films," SCIENCE, vol. 306, pp. 1005-1009, 2004 .

[2] J. Haeni, P. Irvin, W. Chang, R. Uecker, P. Reiche, Y. Li, S. Choudhury, W. Tian, M. Hawley, B. Craigo, A. Tagantsev, X. Pan, S. Streiffer, L. Chen, S. Kirchoefer, J. Levy, and D. Schlom, "Room-temperature ferroelectricity in strained $\mathrm{SrTiO}_{3}$," NATURE, vol. 430, pp. 758-761, 2004.

[3] G. Catalan, A. Janssens, G. Rispens, S. Csiszar, O. Seeck, G. Rijnders, D. Blank, and B. Noheda, "Polar domains in lead titanate films under tensile strain," PHYSICAL REVIEW LETTERS, vol. 96, p. 127602, 2006.

[4] J. O’Donnell, A. Andrus, S. Oh, E. Colla, and J. Eckstein, "Colossal magnetoresistance magnetic tunnel junctions grown by molecular-beam epitaxy," APPLIED PHYSICS LETTERS, vol. 76, pp. 1914-1916, 2000.

[5] Y. Ogimoto, M. Izumi, A. Sawa, T. Manako, H. Sato, H. Akoh, M. Kawasaki, and Y. Tokura, "Tunneling magnetoresistance above room temperature in $\mathrm{La}_{0.7} \mathrm{Sr}_{0.3} \mathrm{MnO}_{3} / \mathrm{SrTiO}_{3} / \mathrm{La}_{0.7} \mathrm{Sr}_{0.3} \mathrm{MnO}_{3}$ junctions," JAPANESE JOURNAL OF APPLIED PHYSICS PART 2-LETTERS, vol. 42, pp. L369-L372, 2003.

[6] M. Bowen, M. Bibes, A. Barthelemy, J. Contour, A. Anane, Y. Lemaitre, and A. Fert, "Nearly total spin polarization in $\mathrm{La}_{2 / 3} \mathrm{Sr}_{1 / 3} \mathrm{MnO}_{3}$ from tunneling experiments," APPLIED PHYSICS LETTERS, vol. 82, pp. 233-235, 2003.

[7] M. Lee, T. Nath, C. Eom, M. Smoak, and F. Tsui, "Strain modification of epitaxial perovskite oxide thin films using structural transitions of ferroelectric $\mathrm{BaTiO}_{3}$ substrate," APPLIED PHYSICS LETTERS, vol. 77, pp. 3547-3549, 2000.

[8] W. Eerenstein, M. Wiora, J. L. Prieto, J. F. Scott, and N. D. Mathur, "Giant sharp and persistent converse magnetoelectric effects in multiferroic epitaxial heterostructures," NATURE MATERIALS, vol. 6, pp. 348-351, 2007.

[9] P. Yu, J. S. Lee, S. Okamoto, M. D. Rossell, M. Huijben, C. H. Yang, Q. He, J. X. Zhang, S. Y. Yang, M. J. Lee, Q. M. Ramasse, R. Erni, Y. H. Chu, D. A. Arena, C. C. Kao, L. W. Martin, and R. Ramesh, "Interface Ferromagnetism and Orbital Reconstruction in $\mathrm{BiFeO}_{3}-\mathrm{La}_{0.7} \mathrm{Sr}_{0.3} \mathrm{MnO}_{3}$ Heterostructures," PHYSICAL REVIEW LETTERS, vol. 105, p. 027201, 2010.

[10] A. Urushibara, Y. Moritomo, T. Arima, A. Asamitsu, G. Kido, and Y. Tokura, "Insulator-metal transition transition and giant magnetoresistance in $\mathrm{La}_{1-x} \mathrm{Sr}_{x} \mathrm{MnO}_{3}$," PHYSICAL REVIEW B, vol. 51, pp. 14103-14109, 1995. 
[11] P. Radaelli, G. Iannone, M. Marezio, H. Hwang, S. Cheong, J. Jorgensen, and D. Argyriou, "Structural effects on the magnetic and transport properties of perovskite $\mathrm{A}_{(1-x)} \mathrm{A}_{(x)} \mathrm{MnO}_{3}(\mathrm{x}=0.25,0.30)$," PHYSICAL REVIEW B, vol. 56, pp. 8265-8276, 1997.

[12] M. Konoto, T. Kohashi, K. Koikie, T. Arima, Y. Kaneko, Y. Tomioka, and Y. Tokura, "Magnetic domain structure of a $\mathrm{La}_{0.7} \mathrm{Sr}_{0.3} \mathrm{MnO}_{3}(001)$ surface observed by a spin-polarized scanning electron microscope," APPLIED PHYSICS LETTERS, vol. 84, pp. 2361-2363, 2004.

[13] A. Khapikov, L. Uspenskaya, I. Bdikin, Y. Mukovskii, S. Karabashev, D. Shulyaev, and A. Arsenov, "Magnetic domains and twin structure of the $\mathrm{La}_{0.7} \mathrm{Sr}_{0.3} \mathrm{MnO}_{3}$ single crystal," APPLIED PHYSICS LETTERS, vol. 77, pp. 2376-2378, 2000.

[14] K. Steenbeck, A. Hiergeist, R. A, and P.-G. L, "Magnetic Anisotropy in $\mathrm{La}_{0.7}(\mathrm{Sr}, \mathrm{Ca})_{0.3} \mathrm{MnO}_{3}$ Epitaxial Thin Films and Crystals," MRS Symposium proceedings, 1999.

[15] C. Kwon, M. Robson, K. Kim, J. Gu, S. Lofland, S. Bhagat, Z. Trajanovic, M. Rajeswari, T. Venkatesan, A. Kratz, R. Gomez, and R. Ramesh, "Stress-induced effects in epitaxial $\left(\mathrm{La}_{0.7} \mathrm{Sr}_{0.3}\right) \mathrm{MnO}_{3}$ films," JOURNAL OF MAGNETISM AND MAGNETIC MATERIALS, vol. 172, pp. 229-236, 1997.

[16] R. Desfeux, S. Bailleul, A. Da Costa, W. Prellier, and A. Haghiri-Gosnet, "Substrate effect on the magnetic microstructure of $\mathrm{La}_{0.7} \mathrm{Sr}_{0.3} \mathrm{MnO}_{3}$ thin films studied by magnetic force microscopy," APPLIED PHYSICS LETTERS, vol. 78, pp. 3681-3683, 2001.

[17] J. Dho, Y. Kim, Y. Hwang, J. Kim, and N. Hur, "Strain-induced magnetic stripe domains in $\mathrm{La}_{0.7} \mathrm{Sr}_{0.3} \mathrm{MnO}_{3}$ thin films," APPLIED PHYSICS LETTERS, vol. 82, pp. 1434-1436, 2003.

[18] K. Steenbeck and R. Hiergeist, "Magnetic anisotropy of ferromagnetic $\mathrm{La}_{0.7}(\mathrm{Sr}$, Ca) ${ }_{0.3} \mathrm{MnO}_{3}$ epitaxial films," APPLIED PHYSICS LETTERS, vol. 75, pp. 1778-1780, 1999.

[19] Z. Wang, G. Cristiani, and H. Habermeier, "Uniaxial magnetic anisotropy and magnetic switching in $\mathrm{La}_{0.67} \mathrm{Sr}_{0.33} \mathrm{MnO}_{3}$ thin films grown on vicinal $\mathrm{SrTiO}_{3}(100)$," APPLIED PHYSICS LETTERS, vol. 82, pp. 3731-3733, 2003.

[20] M. Mathews, F. Postma, J. Lodder, R. Jansen, G. Rijnders, and D. Blank, "Step-induced uniaxial magnetic anisotropy of $\mathrm{La}_{0.67} \mathrm{Sr}_{0.33} \mathrm{MnO}_{3}$ thin films," APPLIED PHYSICS LETTERS, vol. 87, p. 242507, 2005.

[21] E. P. Houwman, G. Maris, G. M. De Luca, N. Niermann, G. Rijnders, D. H. A. Blank, and S. Speller, "Out-of-plane magnetic domain structure in a thin film of $\mathrm{La}_{0.67} \mathrm{Sr}_{0.33} \mathrm{MnO}_{3}$ on $\mathrm{SrTiO}_{3}$ (001) observed by magnetic force microscopy," PHYSICAL REVIEW B, vol. 77, p. 184412, 2008.

[22] L. Néel, "*Anisotropie magnetique superficielle et surstructures dorientation," JOURNAL DE PHYSIQUE ET LE RADIUM, vol. 15, pp. 225-239, 1954.

[23] R. Skomski, Simple models of magnetism. Oxford university press, Oxford, 2008. 
[24] I. Solovyev, N. Hamada, and K. Terakura, "Crucial role of the lattice distortion in the magnetism of $\mathrm{LaMnO}_{3}$," PHYSICAL REVIEW LETTERS, vol. 76, pp. 4825-4828, 1996.

[25] S. Chikazumi, Physics of ferromagnetism. Clarendon Press, Oxford, 2nd ed., 1997.

[26] D. Chuang, C. Ballentine, and R. Ohandley, "Surface and step magnetic-anisotropy," PHYSICAL REVIEW B, vol. 49, pp. 15084-15095, 1994.

[27] Y. Suzuki, H. Hwang, S. Cheong, and R. vanDover, "The role of strain in magnetic anisotropy of manganite thin films," APPLIED PHYSICS LETTERS, vol. 71, pp. 140-142, 1997.

[28] Y. Suzuki, H. Hwang, S. Cheong, T. Siegrist, R. van Dover, A. Asamitsu, and Y. Tokura, "Magnetic anisotropy of doped manganite thin films and crystals," JOURNAL OF APPLIED PHYSICS, vol. 83, pp. 7064-7066, 1998.

[29] F. Tsui, M. Smoak, T. Nath, and C. Eom, "Strain-dependent magnetic phase diagram of epitaxial $\mathrm{La}_{0.67} \mathrm{Sr}_{0.33} \mathrm{MnO}_{3}$ thin films," APPLIED PHYSICS LETTERS, vol. 76, pp. 2421-2423, 2000.

[30] K. Steenbeck, T. Habisreuther, C. Dubourdieu, and J. Senateur, "Magnetic anisotropy of ferromagnetic $\mathrm{La}_{0.7} \mathrm{Sr}_{0.3} \mathrm{MnO}_{3}$ epitaxial thin films: Dependence on temperature and film thickness," APPLIED PHYSICS LETTERS, vol. 80, pp. 3361-3363, 2002.

[31] M. Ziese, H. C. Semmelhack, and P. Busch, "Sign reversal of the magnetic anisotropy in $\mathrm{La}_{0.7} \mathrm{~A}_{0.3} \mathrm{MnO}_{3}(\mathrm{~A}=\mathrm{Ca}, \mathrm{Sr}, \mathrm{Ba})$ films," JOURNAL OF MAGNETISM AND MAGNETIC MATERIALS, vol. 246, pp. 327-334, 2002.

[32] E. P. Houwman, H. Boschker, M. Mathews, H. Nishikawa, G. Koster, G. Rijnders, and D. H. A. Blank, "Magnetic anisotropy in strained thin epitaxial $\mathrm{La}_{0.67} \mathrm{Sr}_{0.33} \mathrm{MnO}_{3}$ films on perovskite substrates described by the Néel anisotropy model," In preparation.

[33] L. Vasylechko, L. Akselrud, W. Morgenroth, U. Bismayer, A. Matkovskii, and D. Savytskii, "The crystal structure of $\mathrm{NdGaO}_{3}$ at $100 \mathrm{~K}$ and $293 \mathrm{~K}$ based on synchrotron data," JOURNAL OF ALLOYS AND COMPOUNDS, vol. 297, pp. $46-52,2000$.

[34] M. Mathews, Structural and magnetic properties of epitaxial $\mathrm{La}_{0.67} \mathrm{Sr}_{0.33} \mathrm{MnO}_{3}$ films and nanostructures. PhD thesis, University of Twente, 2007.

[35] H. Nishikawa, E. Houwman, H. Boschker, M. Mathews, D. H. A. Blank, and G. Rijnders, "Rotation of the magnetic easy axis in $\mathrm{La}_{0.67} \mathrm{Sr}_{0.33} \mathrm{MnO}_{3}$ thin film on $\mathrm{NdGaO}_{3}(112)$," APPLIED PHYSICS LETTERS, vol. 94, p. 042502, 2009.

[36] H. Boschker, M. Mathews, E. P. Houwman, H. Nishikawa, A. Vailionis, G. Koster, G. Rijnders, and D. H. A. Blank, "Strong uniaxial in-plane magnetic anisotropy of (001)- and (011)-oriented $\mathrm{La}_{0.67} \mathrm{Sr}_{0.33} \mathrm{MnO}_{3}$ thin films on $\mathrm{NdGaO}_{3}$ substrates," PHYSICAL REVIEW B, vol. 79, p. 214425, 2009.

[37] Y. Tokura, "Features of cmr manganites," in Colossal magnetoresistive oxides (Y. Tokura, ed.), pp. 1-52, Gordon and Breach Science Publishers, the Netherlands, 2000. 
[38] R. Skomski, "The itinerant limit of metallic anisotropy," IEEE TRANSACTIONS ON MAGNETICS, vol. 32, pp. 4794-4796, 1996.

[39] D. S. Dessau and Z. X. Shen, "Direct electronic structure measurements of the colossal magnetoresistive oxides," in Colossal magnetoresistive oxides (Y. Tokura, ed.), pp. 149-185, Gordon and Breach Science Publishers, the Netherlands, 2000.

[40] A. Miniotas, A. Vailionis, E. Svedberg, and U. Karlsson, "Misfit strain induced lattice distortions in heteroepitaxially grown $\mathrm{La}_{x} \mathrm{Ca}_{1-x} \mathrm{MnO}_{3}$ thin films studied by extended x-ray absorption fine structure and high-resolution x-ray diffraction," JOURNAL OF APPLIED PHYSICS, vol. 89, pp. 2134-2137, 2001.

[41] H. Hwang, S. Cheong, P. Radaelli, M. Marezio, and B. Batlogg, "Lattice effects on the magnetoresistance in doped $\mathrm{LaMnO}_{3}$, PHYSICAL REVIEW LETTERS, vol. 75 , pp. 914-917, 1995. 


\title{
Chapter 5
}

\section{Interface engineering at the $\mathrm{La}_{0.67} \mathrm{Sr}_{0.33} \mathrm{MnO}_{3} / \mathrm{SrTiO}_{3}$ interface}

\begin{abstract}
The use of $\mathrm{La}_{0.67} \mathrm{Sr}_{0.33} \mathrm{MnO}_{3}$ (LSMO) in spintronic devices requires spin polarized conductivity at the interface. Due to interface reconstructions, generally the spin polarized conductivity is reduced or completely absent at the interface. Interface engineering can therefore be a valuable tool to study and improve the interfacial properties. Here, three strategies for the optimization of the LSMO at the interface are presented. The first one is based on analysis of the interface in terms of the recently developed polar discontinuity model for oxide interfaces. Based on the model, the insertion of a single atomic layer of $\mathrm{La}_{0.33} \mathrm{Sr}_{0.67} \mathrm{O}$ is proposed and experimentally realized. The results show improved properties, due to a significant reduction of interdiffusion at the engineered interface. An alternative approach is to study interfaces in the (011) crystal direction. It is shown that (011) LSMO has different properties in comparison to (001) oriented LSMO, with reduced conductivity, but enhanced magnetization. Finally, the effect of chemical order and disorder in the system was studied. To obtain a chemically ordered LSMO interface, a layer of $\mathrm{SrMnO}_{3}$ was inserted, which was doped with the use of modulation doping from donor states in the STO. Preliminary results show that the modulation doping is succesfull, although the effect of the chemical order on the magnetization and conductivity at the interface was small. All three modifications did not result in the desired fully spin polarized conductivity at the interfaces. Therefore, it is concluded that the reduction of the properties at the interface is due to the discontinuity in the oxygen octahedra rotations at the interface, as this is the only variable which was not controlled during the experiments.
\end{abstract}




\subsection{Introduction}

$\mathrm{La}_{0.67} \mathrm{Sr}_{0.33} \mathrm{MnO}_{3}$ (LSMO) is an interesting material for application in spintronic devices. Examples are magnetic tunnel junctions [1-4], Schottky devices [5-7] and magnetoelectric devices [8-13]. Furthermore, LSMO is also used to investigate other materials, e.g. by spin injection into cuprate superconductors [14-17], to probe spin polarization at the $\mathrm{BaTiO}_{3} / \mathrm{Fe}$ interface [18] and to study spin dependent transport in organic materials [19]. The material requirements for these devices are quite similar; the spin polarized conductivity, and therefore the magnetism as well, should be maintained at the interface of the LSMO. For convenience, the magnetic tunnel junction (MTJ) is taken as a prototypical device, as here the device performance directly represents LSMO's interfacial spin polarization.

Several examples of LSMO tunnel junctions have been presented in literature [1-4], with a maximum obtained tunnel magnetoresistance ratio (TMR) of 4000, which corresponds to a spin polarization of more than 95\%. However, the TMR drops rapidly with temperature and the devices cannot be prepared very reproducibly. The interface is thought to be the cause of the less ideal behaviour. At the interface between LSMO and the barrier material, e.g. $\mathrm{SrTiO}_{3}$ (STO), the magnetization and conductivity of the LSMO is significantly reduced. This main problem of LSMO tunnel devices is illustrated in figure 5.1, which presents temperature dependent magnetization measurements at three different lengthscales, from bulk sensitive to surface sensitive. It is clear that the surface magnetization decreases much more rapidly with temperature than the bulk magnetization.

Three different explanations for the reduced properties at LSMO surfaces and interfaces are given in literature. First, the change in properties might be due to a difference in doping at the interface [20-23]. Secondly, an orbital reconstruction has been observed at the interface, which whould favour an antiferromagnetic state [24]. And finally, the change in oxygen octahedra rotation at the interface results, in theory, in Mn off-centering and therefore in a change in the spin-spin interaction [25]. A structural expansion of the unit cell at the surface [26] has been observed, which might be related to a change in the oxygen octahedra rotations. In order to identify the mechanism of the reduction of the spin polarized conductivity at the interface, further measurements are therefore required.

Interface engineering is an interesting tool for the study of interfaces. It allows for direct intervention at the interface during growth and subsequently the effect of the interventions can be studied. A first attempt to use interface engineering to study and improve the properties of LSMO was made by Yamada et al., who inserted a 2 unit cell thick $\mathrm{LaMnO}_{3}$ (LMO) layer at the interface and found an improvement in the magnetization [27]. However, no improvement in TMR device performance was obtained [28]. The LMO layer changes the charge state at the interface towards the ferromagnetic insulating region of the phase diagram. It is therefore likely that the interface engineering with LMO improves the magnetization, but not the conductivity at the interface.

Here, three alternative strategies for the optimization of the LSMO/STO interface are investigated. The first one is based on analysis of the interface in terms of the 


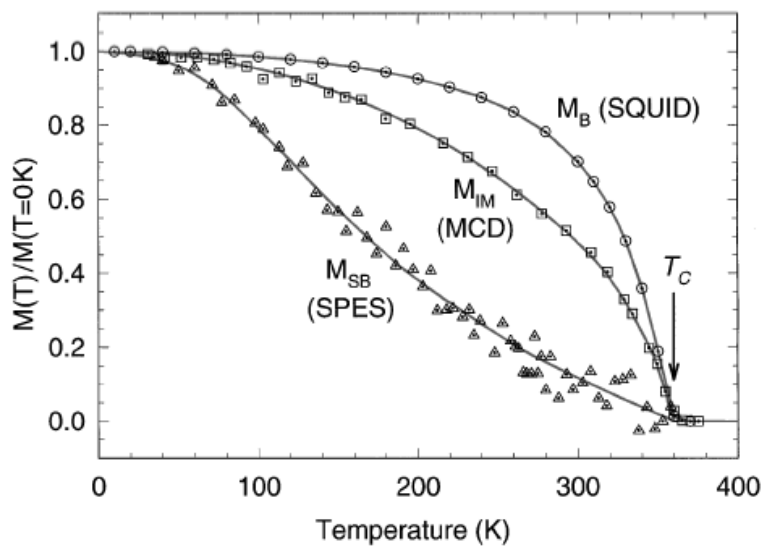

Figure 5.1: The temperature dependence of magnetization of a $190 \mathrm{~nm}$ LSMO layer measured at three different length scales. The superconducting quantum interference device (SQUID) measures the bulk magnetization $\left(M_{\mathrm{B}}\right)$. The other two techniques are surface sensitive. X-ray magnetic circular dichroism (MCD) has an intermediate length scale of $5 \mathrm{~nm}\left(M_{\mathrm{IM}}\right)$ and spin-resolved photoemission spectroscopy (SPES) is very surface sensitive with a length scale of $0.5 \mathrm{~nm}\left(M_{\mathrm{SB}}\right)$. This figure has been reproduced with permission from the author from [34]. (c) 1998 the American Physical Society.

polar discontinuity model for oxide interfaces $[29,30]$. The LSMO/STO interface is polar and therefore reconstructions at the interface are expected. The polar discontinuity can be compensated with the use of interface engineering, allowing experimental investigation of this effect. Secondly, it was proposed that the crystal orientation of the interface affects the properties as well [31-33]. Therefore, interfaces in the $(011)_{\mathrm{pc}}$ direction are studied. (The subscripts c, o and pc are used to indicate the cubic, orthorhombic and pseudocubic crystal structures, respectively.) It is shown that $(011)_{\mathrm{pc}}$ LSMO has different properties in comparison to $(001)_{\mathrm{pc}}$ oriented LSMO with reduced conductivity but nearly perfect magnetization at the interfaces. Finally, the effect of chemical order and disorder in the system was studied. To obtain a chemically ordered LSMO interface, a layer of $\mathrm{SrMnO}_{3}$ was inserted, which was doped with the use of modulation doping from donor states in the STO.

This chapter is organized as follows. In the first section, the experimental method to study the interface properties is described. It is proposed that the investigation of the thickness dependence of thin LSMO layers can be used to study the properties of the LSMO/STO interfaces. In the following sections, the results on polar discontinuities at the interface, $(011)_{\mathrm{pc}}$ oriented interfaces and ordered versus disordered interfaces are presented successively. The chapter ends with a discussion about the implications of the experiments for the mechanism of the reduction of the properties at LSMO interfaces. 

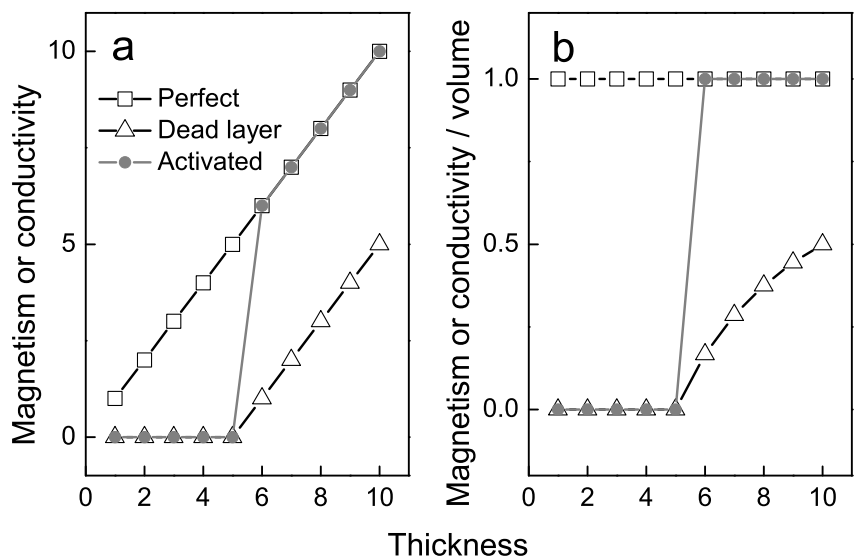

Figure 5.2: The expected thickness dependence of functional properties for three cases: perfect interfaces (squares), the dead layer (triangle) and activated behaviour (circles).

\subsection{Experimental method for the study of inter- face properties}

For the proposed experiments in this chapter, a method to investigate the magnetization and conductivity at the LSMO interface is required. Rather than direct measurements of the interface properties [27], here the thickness dependence of the bulk sample properties is measured. When the thickness of the LSMO layer is decreased, the interfacial properties will become more important compared to the bulk properties and therefore, it is possible to obtain information about the interfaces without advanced interfacial measurement techniques.

In figure 5.2, the expected thickness dependence of LSMO is shown for three limiting cases of the interfacial properties. The first curve (black squares) represents LSMO films with perfect interfaces and the functional properties are maintained down to the single unit cell layer film. The second curve (black triangles) represents LSMO thin films with an interfacial dead layer. The thickness of the dead layer does not depend on the film thickness and the curve is shifted with repect to the first curve. The critical thickness is defined as the thickness at which the functional properties disappear. The critical thickness is a measure for the thickness of the dead layer at the interfaces. The last curve (grey diamonds) indicates an activated behaviour, where the films are dead up to a certain thickness and then become fully functional including all interfacial layers.

LSMO thin films are well known for their dead layer behaviour. This dead layer is defined as the thickest layer for which both conductivity and ferromagnetism are 
absent. Early reports have mentioned a dead layer thickness of 3, 4, 4 and $5 \mathrm{~nm}$ for films grown on $\mathrm{NdGaO}_{3}(110)_{\mathrm{o}}$, STO $(001)_{\mathrm{c}}, \mathrm{MgO}(001)_{\mathrm{c}}$ and $\mathrm{LaAlO}_{3}(001)_{\mathrm{pc}}$ [35-37] respectively. Later on, Huijben et al. [38] showed that different critical thicknesses for the electrical transport and ferromagnetic properties exist. For LSMO samples grown on STO, the dead layer is reported to be 8 unit cells, while the samples remain ferromagnetic up to 3 unit cell thick films. Similar results have also been published by Kim et al. [39].

Therefore, the modifications of the LSMO proposed with the interface engineering should ideally result in a change from dead layer behaviour to either perfect interfaces or activated behaviour. Also, a reduction of the dead layer thickness indicates an improvement in the interfacial properties. As the dead layer for conductivity is larger, especially the conductivity at the interfaces should be improved.

\subsection{Polar discontinuities}

Recently, Ohtomo and Hwang proposed a model to describe charge transfer at oxide heterointerfaces [29]. The model predicts charge tranfer to occur if a polar discontinuity is present at the interface in combination with a multi-valent metal ion. This model was used to explain the difference in electronic properties of $\mathrm{LaAlO}_{3} / \mathrm{STO}$ heterointerfaces based on $\mathrm{SrO}$ and $\mathrm{TiO}_{2}$ terminated $\mathrm{SrTiO}_{3}$, as discussed in detail in chapter 2. The hole doping of the LSMO layer [20], due to the presence of the STO, can also be understood in terms of this polar discontinuity driven electronic reconstruction.

Here, the LSMO/STO interfaces are analyzed in terms of an electronically reconstructed polar discontinuity model. The Thomas Fermi screening length of LSMO is $\approx 0.31 \mathrm{~nm}[7]$, which justifies the use of a model in the ionic limit for a metallic compound. Furthermore, the general physics at oxide interfaces can be understood by finding the charge density at each layer using electrostatics and then the electron density determines the properties of each different layer [40, 41]. Therefore, the model will be used to calculate the interfacial electron density and the properties of the interfaces can be predicted by refering to the LSMO phase diagram at the appropriate electron density. The main conclusion is that the electronic reconstructions depend on the atomic stacking sequence at the interface, the $\mathrm{MnO}_{2}$ terminated interface is overdoped and the $\mathrm{La}_{0.67} \mathrm{Sr}_{0.33} \mathrm{O}$ terminated interface is underdoped. An interface configuration in which the manganese valence state is not changed after the reconstruction is possible. This configuration can be realized with careful engineering of the $A$-site cation distribution.

The experimental results of thin LSMO films with the different interface configurations are described in the next part. Using scanning transmission electron microscopy (STEM), it is shown that the samples with the polar discontinuity are intermixed over a distance of 2 uc. In contrast, the samples with engineered interfaces, and therefore without the polar discontinuity, have a sharp interface. The latter samples have improved magnetization and conductivity compared to the heterostructures without the engineered interfaces. 


\subsubsection{LSMO/STO interface configurations}

Two different atomic stacking sequences are possible for the LSMO/STO interfaces, due to the possible $A \mathrm{O}$ or $\mathrm{BO}_{2}$ termination of each material. One will be referred to as the $\mathrm{La}_{0.67} \mathrm{Sr}_{0.33} \mathrm{O}$ terminated interface and the other as the $\mathrm{MnO}_{2}$ terminated interface. The $\mathrm{La}_{0.67} \mathrm{Sr}_{0.33} \mathrm{O}$ terminated interface is shown in figure 5.3a. The STO (LSMO) layers are at the negative (positive) $x$ coordinates. At this interface the atomic stacking sequence is $\mathrm{SrO}(-2)-\mathrm{TiO}_{2}(-1)-\mathrm{La}_{0.67} \mathrm{Sr}_{0.33} \mathrm{O}(0)$ $\mathrm{MnO}_{2}(1)$. The number between the parentheses indicates the $x$ coordinate of the layer. The $\mathrm{MnO}_{2}$ terminated interface is shown in figure 5.3c. Here the atomic stacking sequence is $\mathrm{TiO}_{2}(-1)-\mathrm{SrO}(0)-\mathrm{MnO}_{2}(1)-\mathrm{La}_{0.67} \mathrm{Sr}_{0.33} \mathrm{O}(2)$. An alternative stacking sequence is shown as well, figure 5.3e, in which a single atomic layer of $\mathrm{La}_{0.33} \mathrm{Sr}_{0.67} \mathrm{O}$ is inserted between the two materials. This gives an atomic stacking sequence at the interface of $\mathrm{SrO}(-2)-\mathrm{TiO}_{2}(-1)-\mathrm{La}_{0.33} \mathrm{Sr}_{0.67} \mathrm{O}(0)-\mathrm{MnO}_{2}-\mathrm{La}_{0.67}(1)-$ $\mathrm{Sr}_{0.33} \mathrm{O}(2)$.

The different interface configurations are analyzed in terms of the model for the electronic reconstruction at polar discontinuities. The atomic layers of the STO are charge neutral, whereas LSMO consists of $\mathrm{La}_{0.67} \mathrm{Sr}_{0.33} \mathrm{O}$ and $\mathrm{MnO}_{2}$ layers with a respective charge of $\frac{2}{3} e-/ \mathrm{uc}$ and $-\frac{2}{3} e-/ \mathrm{uc}$, in the ionic limit. The $\mathrm{Ti}^{4+}$ valence state is stable at these interfaces, as the $\mathrm{Ti}_{2 g}$ levels are $1 \mathrm{eV}$ higher in energy compared with the Mn $\mathrm{e}_{g}$ levels [20]. All free electrons will occupy the lower lying Mn states. As LSMO is metallic, no bandgap prevents charge transfer and an electronic reconstruction is expected without a critical thickness.

The resulting potential and charge distribution at the $\mathrm{La}_{0.67} \mathrm{Sr}_{0.33} \mathrm{O}$ terminated interface is shown in figure 5.3b. The net charge at the first $\mathrm{MnO}_{2}$ layer is $-1 e-$ / uc and therefore, the $\mathrm{Mn}$ valence state is $\mathrm{Mn}^{3+}$. The second $\mathrm{MnO}_{2}$ layer has a net charge of $-\frac{2}{3} e$ - /uc, which corresponds to optimal doping. So, the Mn ions in the first layer at the interface are underdoped. From the LSMO phase diagram [42], it can be expected that this layer is insulating and antiferromagnetic. For the $\mathrm{MnO}_{2}$ terminated interface, the polarity of the potential buildup and reconstruction is reversed. The net charge at the first $\mathrm{MnO}_{2}$ layer is $-\frac{1}{3} e$ - $/ \mathrm{uc}$, which corresponds to an overdoped $\mathrm{Mn}$ valence state of $\mathrm{Mn}^{3.67+}$. In the phase diagram, a charge ordered insulating state is present at this doping level. So, this interface is expected to be insulating and not ferromagnetic either. Note that a similar analysis of the electron density at the interface was presented by Hikita et al. [7], in order to explain the termination dependent band offset differences in Schottky devices. Finally, the interface engineered stacking with the single $\mathrm{La}_{0.33} \mathrm{Sr}_{0.67} \mathrm{O}$ atomic layer does not result in a diverging potential, as the net charge of $\frac{1}{3} e$ - $/ \mathrm{uc}$ in the $\mathrm{La}_{0.33} \mathrm{Sr}_{0.67} \mathrm{O}$ layer is exactly the amount of charge required to screen the potential buildup. Therefore, in this case, the first $\mathrm{MnO}_{2}$ layer is optimally doped. All interface configurations have a band offset, which is largest for the $\mathrm{La}_{0.67} \mathrm{Sr}_{0.33} \mathrm{O}$ terminated interface. 
$\mathrm{a}$

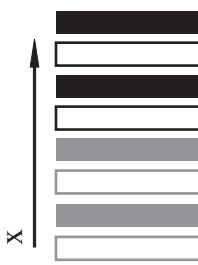

$\mathrm{c}$
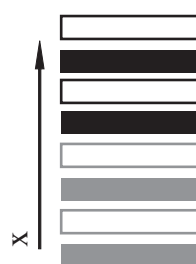

e

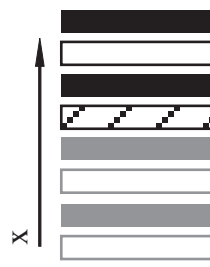

$$
\begin{aligned}
& \left(\mathrm{MnO}_{2}\right)^{-0.67} \\
& \left(\mathrm{MnO}_{2}\right)^{-1} \\
& \left(\mathrm{TiO}_{2}\right)^{0} \\
& \left(\mathrm{TiO}_{2}\right)^{0}
\end{aligned}
$$$$
\begin{aligned}
& \left(\mathrm{La}_{0.67} \mathrm{Sr}_{0.33} \mathrm{O}\right)^{0.67} \\
& \left(\mathrm{La}_{0.67} \mathrm{Sr}_{0.33} \mathrm{O}\right)^{0.67}
\end{aligned}
$$$$
(\mathrm{SrO})^{0}
$$$$
(\mathrm{SrO})^{0}
$$

b

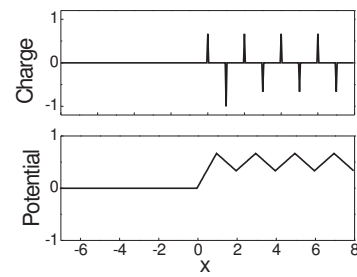

d

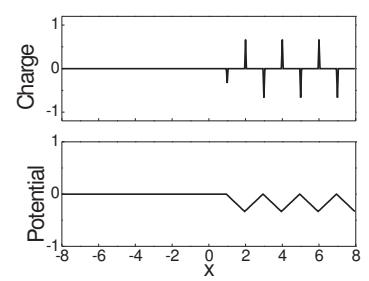

f

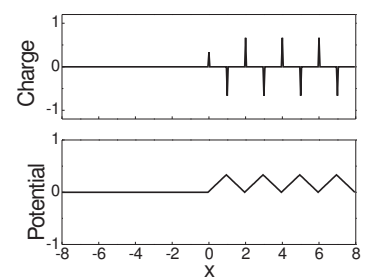

Figure 5.3: The LSMO/STO interface configurations. a \& b) $\mathrm{La}_{0.67} \mathrm{Sr}_{0.33} \mathrm{O}$ terminated LSMO/STO interface. c \& d) $\mathrm{MnO}_{2}$ terminated LSMO/STO interface. e \& f) $\mathrm{TiO}_{2} / \mathrm{La}_{0.33} \mathrm{Sr}_{0.67} \mathrm{O} / \mathrm{MnO}_{2} \mathrm{LSMO} / \mathrm{STO}$ interface. Left) Schematic representation. Right) Charge distribution and potential after reconstruction. 

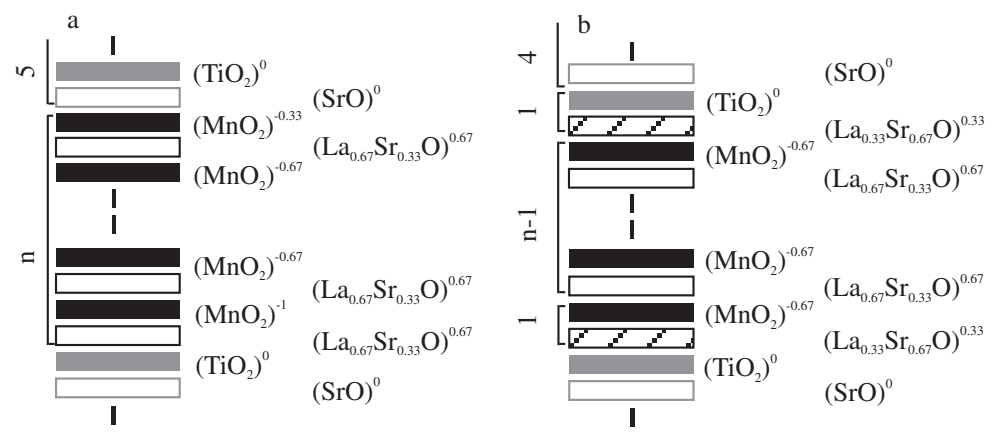

Figure 5.4: Atomic stacking sequence at the interfaces for heterostructures grown on STO substrates. a) $n$ LSMO and 5 STO. b) $1 \mathrm{La}_{0.33} \mathrm{Sr}_{0.67} \mathrm{MnO}_{3}$, $n$-1 LSMO, $1 \mathrm{La}_{0.33} \mathrm{Sr}_{0.67} \mathrm{TiO}_{3}$ and 4 STO. The net charge at each layer is indicated. In a) one of the interfaces is overdoped and the other is underdoped. In b) the Mn doping is constant in the entire structure.

\subsubsection{Experimental}

All heterostructures were grown with pulsed laser deposition (PLD) at $800{ }^{\circ} \mathrm{C}$ in an oxygen environment of 0.27 mbar, as described in detail in chapter $3 . \mathrm{TiO}_{2}$ terminated [43] STO substrates were used. The LSMO layer thickness was variable, while the STO layer thickness was fixed at 5 unit cells. In order to realize the interface engineered heterostructure, figure $5.4 \mathrm{~b}$, first a single unit cell layer of $\mathrm{La}_{0.33} \mathrm{Sr}_{0.67} \mathrm{MnO}_{3}$ was grown. This was followed with the growth of $n$ - 1 layers of LSMO, then a single unit cell layer of $\mathrm{La}_{0.33} \mathrm{Sr}_{0.67} \mathrm{TiO}_{3}$ and finally, 4 unit cell layers of STO. This recipe resulted in heterostructures with $n$ layers of $\mathrm{Mn}$ ions, which can therefore be compared with the $n$ unit cell layer LSMO/STO structure without the interface engineering.

In figure 5.5, the RHEED specular spot intensity during the deposition of the $n=8$ samples without and with interface engineering is shown. The clear oscillations of the intensity suggest layer-by-layer growth and allow for the possibility to grow single unit cell layers. After the growth of each layer, the RHEED image showed clear two dimensional spots, indicating difraction from smooth crystalline surfaces. Figure 5.5 also shows the full width at the half maximum (FWHM) of the specular spot intensity, measured along the $(10)_{\mathrm{pc}}$ direction. Clear oscillations are observed, especially for the interface engineered sample. For the interface engineered sample, the FWHM after each unit cell layer was equal to that of the original substrate reflection, while for the non interface engineered sample, an increase in FWHM was observed. This indicates a higher quality layer-by-layer growth of the interface engineered sample. However, the higher quality growth of the interface engineered sample was observed in some, but not all, of the sets of samples fabricated. The smooth surface topography of the samples was confirmed with atomic force microscopy (AFM) measurements. 

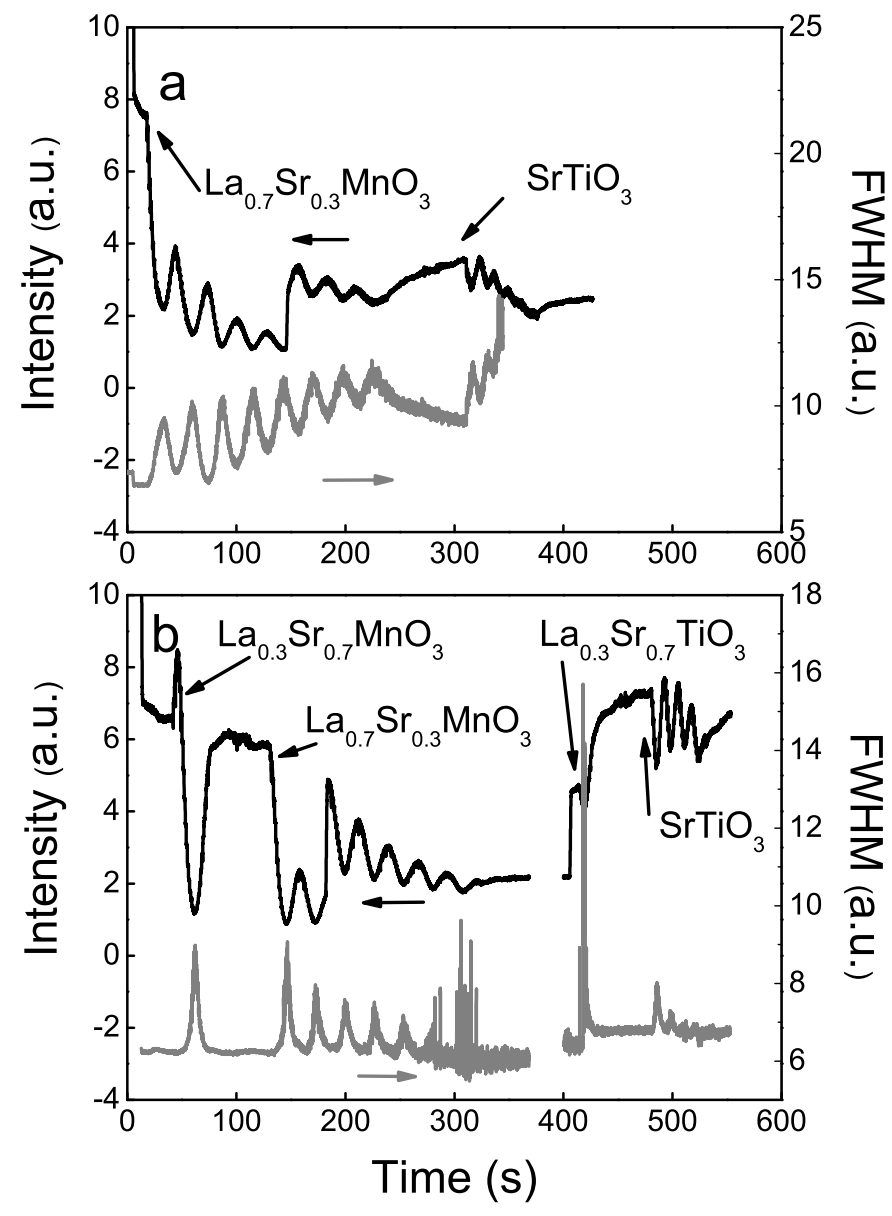

Figure 5.5: RHEED specular spot intensity oscillations and FWHM during the growth of the $n=8 \mathrm{LSMO} / \mathrm{STO}$ heterostructures with and without interface engineering. The starting point of each layer is indicated. a) Without interface engineering. The intensity was increased manually at $t=140 \mathrm{~s}$. b) With interface engineering. The intensity was increased manually at $t=180 \mathrm{~s}$ and $t=405 \mathrm{~s}$. The increase of the RHEED intensity at the start of the growth of the $\mathrm{La}_{0.33} \mathrm{Sr}_{0.67} \mathrm{MnO}_{3}$ layer is attributed to the presence of small unit cell deep holes on the substrate surface. 
a

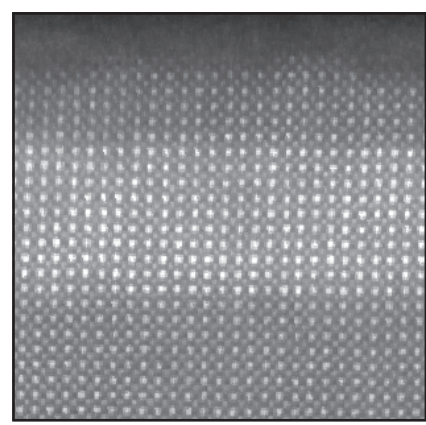

c

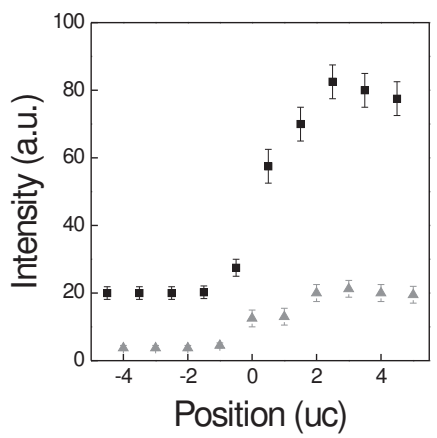

b

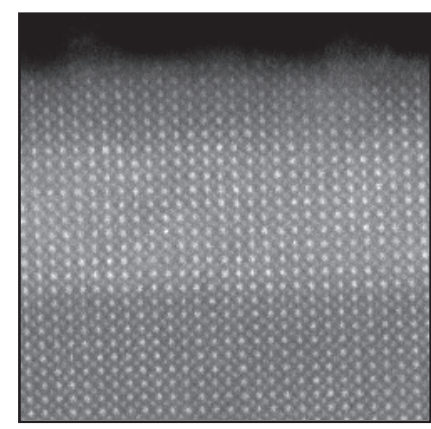

d

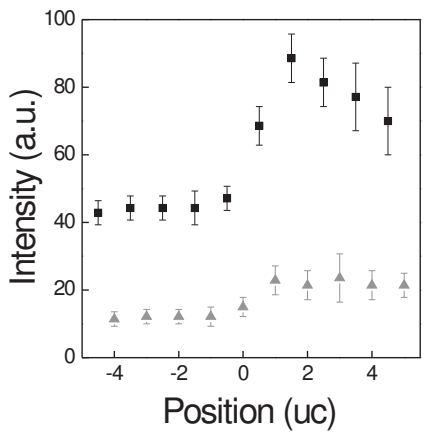

Figure 5.6: STEM measurements of the $n=10$ samples. The HAADF images are shown in the top graphs, while the layer resolved intensity analysis of the substrate film interfaces is shown in the bottom graphs. The nominal interfacial $\mathrm{TiO}_{2}$ layer is at position 0 and black squares (grey triangles) indicate $A$-site ( $B$-site) layers. a) and c) Non interface engineered sample. b) and d) Interface engineered sample.

\subsubsection{Results}

\section{STEM measurements}

High angle annular dark field (HAADF) STEM images of the non interface engineered and the interface engineered $n=10$ samples are shown in figures $5.6 \mathrm{a}$ and $5.6 \mathrm{~b}$. The intensity of each atomic column is quantitatively analyzed [44] and the average intensity in each layer is shown in figures 5.6c and $5.6 \mathrm{~d}$ for the substrate film interface (bottom interface). For the non interface engineered sample, the $A$-site cations are intermixed. The observed intensity at $z=1 / 2$ is approximately the average of the expections for the $\mathrm{SrO}$ and $\mathrm{La}_{0.67} \mathrm{Sr}_{0.33} \mathrm{O}$ layers. Deviations from the expected intensity are also observed at $z=-1 / 2$ and $z=3 / 2$. A similar intermixing is observed at the $B$-site ions. The two layers at $z=0$ and $z=1$ are intermixed. 
In contrast to the intermixing observed for the sample with the polar discontinuity at the interface, the interface engineered sample, figure 5.6d, has less intermixing. Only the intensity of the layer at $z=1 / 2$ has intermediate intensity. This is expected, as this is the layer with the nominal composition of $\mathrm{La}_{0.33} \mathrm{Sr}_{0.67} \mathrm{O}$. The layer at $z=0$ is attributed to $\mathrm{TiO}_{2}$ as the error bars between the points at $z=0$ and $z=-1$ overlap.

A similar analysis could not be made for the top interface, due to difficulty with obtaining a specimen with constant thickness up to the surface of the film. It is noted that away from the interface in the LSMO, the intensity of both the $A$ site and $B$-site layers, see figures $5.6 \mathrm{c}$ and $5.6 \mathrm{~d}$, decreases in both samples. This can be explained by assuming the specimen is thinner at the top. The samples were also investigated with electron energy loss spectroscopy (EELS). Composition maps based on the EELS intensity support the observations in the HAADF STEM measurements. The bottom interface in the non interface engineered sample has more intermixing than the interface engineered sample. Furthermore, it was observed that the top interfaces of both samples were less intermixed than the bottom interface of the non interface engineered sample.

EELS measurements can be used to analyze the valence states of the ions in the sample. At the LSMO/STO interface, both Ti and Mn might show a change in valence, due to a local change in doping. No changes between the bulk Mn and the interfacial Mn spectra were observed. The Mn L23 edge shifts linearly in energy as a function of the Mn valence state [45]. Based on the calculations in the polar discontinuity model, a valence change of $\frac{1}{3} e$ - is expected at the interface. This would result in a shift of $0.8 \mathrm{eV}$ in the spectra, which was not observed in both samples. No changes were observed in the Ti valence state at the interface either.

\section{Magnetization and resistivity measurements}

The magnetization measurements of the $n=5,6,7 \& 8$ samples are presented in figure 5.7. The field was applied in-plane along the $[100]_{c}$ STO crystal direction. For all thicknesses, the interface engineering resulted in a higher $T_{\mathrm{C}}$ and a higher saturation magnetization. The method to determine the $T_{\mathrm{C}}$ of the samples is discussed in appendix A. The observed magnetization for both interface engineered and non-interface engineered samples is larger with respect to reports of LSMO layers without the STO capping layer [38, 39]. The $n=7$ samples differ from the others, as here a higher saturation magnetization is observed and the magnetization curve approaches $T_{\mathrm{C}}$ linearly ${ }^{1}$.

Figure 5.8 presents the temperature dependence of the resisitivy of the $n=$ $5,6,7 \& 8$ samples. For all thicknesses, the interface engineering results in a lower resistivity curve. The $n=5$ samples and the non interface engineered $n=6$ sample are insulating at low temperature. The $n=6$ interface engineered sample has an upturn in the resisitivity at low temperature but conductivity was observed down to the lowest temperature $(10 \mathrm{~K})$ in the measurement.

\footnotetext{
${ }^{1}$ It is unclear why the $n=7$ samples are different, but it is noted that they were grown at a slightly higher deposition temperature and using a different heater.
} 


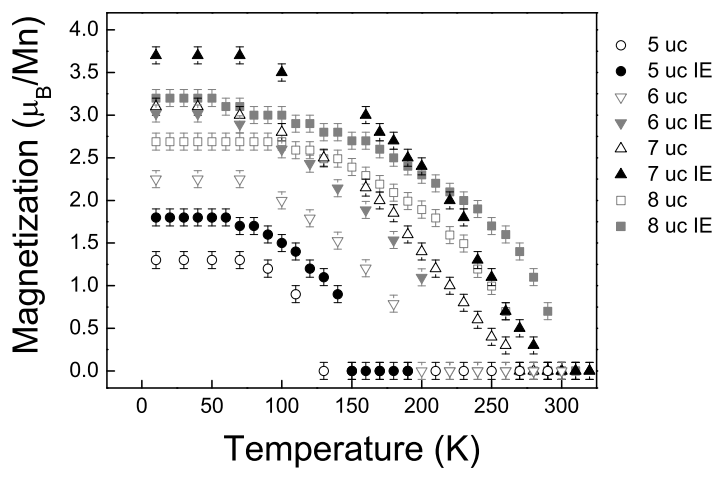

Figure 5.7: Temperature dependent magnetization measurements. Comparison between samples with (IE) and without interface engineering.

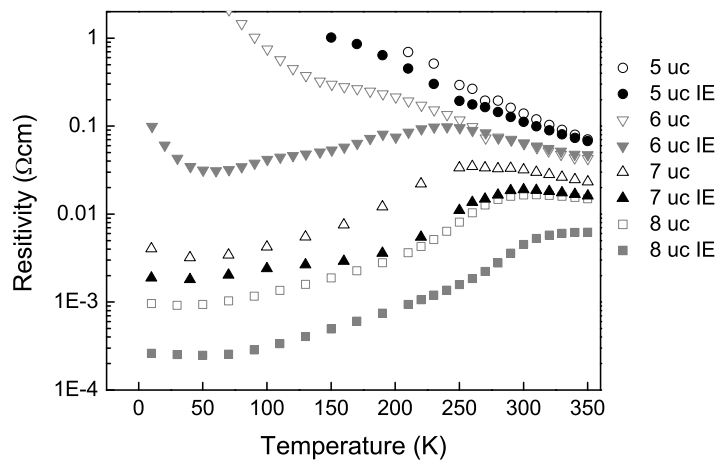

Figure 5.8: Temperature dependent resistivity measurements. Comparison between samples with (IE) and without interface engineering. 

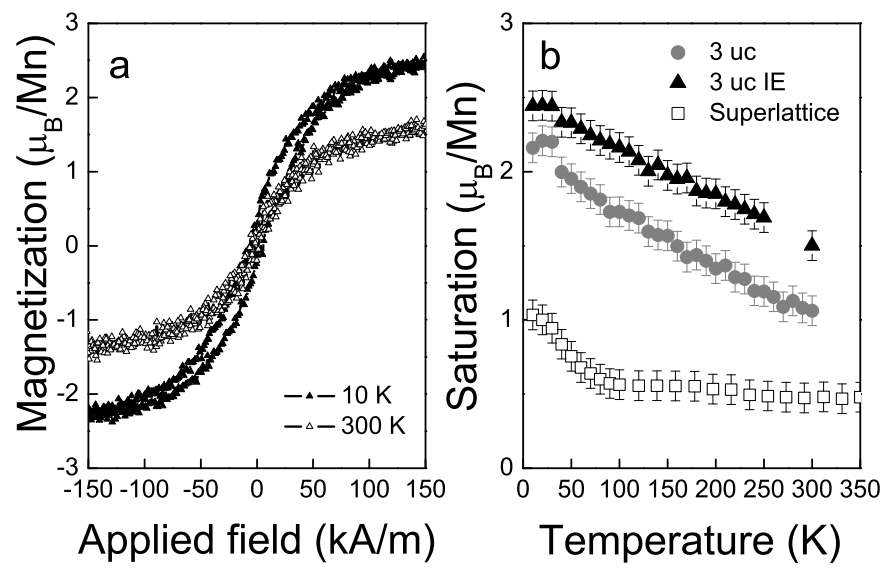

Figure 5.9: Magnetization of the $n=3$ samples. a) Magnetization loops of the interface engineered sample at different temperatures. A remanent magnetization is still observed at $300 \mathrm{~K}$. b) Temperature dependence of the magnetization. The magnetization of a superlattice with three repetitions of 3 uc LSMO and 5 uc STO is shown as well.

\section{Anomalous magnetization in the $n=3$ samples}

The expected ferromagnetic dead layer is 3 uc. For the $n=3$ samples, however, an anomalous magnetization was observed in this study. Figure 5.9a presents the magnetic hysteresis loops of the $n=3$ sample with engineered interfaces. The low temperature saturation magnetization is $2.5 \mu_{\mathrm{B}} / \mathrm{Mn}$, which is significantly larger than the saturation magnetization of the $n=5$ samples. At room temperature, remanent magnetization is still observed, indicating that $T_{\mathrm{C}}$ is larger than room temperature. The temperature dependence of the saturation magnetization of the two $n=3$ samples is shown in figure $5.9 \mathrm{~b}$. The magnetization decreases linearly with temperature.

The increase of $T_{\mathrm{C}}$ is reminiscent of the results obtained by Sadoc et al. on $\mathrm{LSMO} / \mathrm{BaTiO}_{3}$ superlattices. Therefore, an $(\mathrm{LSMO})_{3}(\mathrm{STO})_{3}$ superlattice with three repetitions was grown and measured as well. The magnetic behaviour of the superlattice is qualitatively different from the single layer samples. The saturation magnetization at $10 \mathrm{~K}$ is approximately a factor of two smaller and decreases rapidly until $T=100 \mathrm{~K}$. Above $100 \mathrm{~K}$, the magnetization is almost independent on the temperature at $0.5 \mu_{\mathrm{B}} / \mathrm{Mn}$. 


\subsubsection{Discussion}

The main result of this research is the improvement of the properties with the use of interface engineering. The samples for which the polar discontinuities at the interfaces were artificially removed showed less intermixing at the interfaces, especially the film substrate interface, and improved magnetization and conductivity compared to the non interface engineered samples. Therefore, the improvement in functional properties can be attributed to the reduction of the intermixing in the interface engineered samples.

Intermixing is expected at polar interfaces as intermixing can reduce the electrostatic band offset [30]. Significant intermixing was only observed at the bottom interface of the non interface engineered sample, which corresponds to the $\mathrm{La}_{0.67} \mathrm{Sr}_{0.33} \mathrm{O}$ terminated interface shown in figure 5.3a. This interface configuration has the largest band offset and therefore, the observations of the intermixing are in good agreement with the model. The expected valence changes of $\mathrm{Mn}$ at the $\mathrm{MnO}_{2}$ and $\mathrm{La}_{0.67} \mathrm{Sr}_{0.33} \mathrm{O}$ terminated interfaces, however, are not observed. This suggests that the polar discontinuity is not compensated with an electronic reconstruction, but with a structural reconstruction.

Furthermore, it is noted that the non interface engineered samples have a higher $T_{\mathrm{C}}$, larger spontaneous magnetization and larger conductivity in comparison to the samples discussed in the literature [38, 39], although in superlattices an even larger improvement of the functional properties was reported [46, 47]. This improvement is attributed to the presence of the STO capping layer in the non interface engineered samples. Due to the coherent heteroepitaxy, the oxygen octahedra structure is continued across the interface and a structural LSMO surface reconstruction can be prevented. The improved properties due to the capping layer are in good agreement with the x-ray photoemission spectroscopy (XPS) data presented in chapter 3. The XPS measurements show a more homogeneous electronic structure in the capped samples.

Finally, the anomalous magnetization of the $n=3$ samples is discussed. Similar results were obtained by Sadoc et al. in LSMO $)_{3}\left(\mathrm{BaTiO}_{3}\right)_{3}$ superlattices with a $T_{\mathrm{C}}$ of $650 \mathrm{~K}$ [48]. This effect was explained with the control of the orbital configuration of the LSMO in the superlattice geometry. The out-of-plane oxygen octahedra rotations are minimized due to the coupling with the $\mathrm{BaTiO}_{3}$ and the tensile strain subsequently favours the $\mathrm{d}_{\mathrm{x}^{2}-\mathrm{y}^{2}}$ orbitals. This results in strong in-plane ferromagnetic coupling and enhancement of $T_{\mathrm{C}}$. Especially the magnetization of the LSMO/STO superlattice is in qualitative agreement with their results.

\subsubsection{Conclusions}

The LSMO/STO interface is polar and therefore an interface reconstruction must occur. With the insertion of a single $\mathrm{La}_{0.33} \mathrm{Sr}_{0.67} \mathrm{O}$ atomic layer at the interface, the polar discontinuity is compensated and the reconstruction is prevented. Measurements on ultrathin LSMO layers without polar discontinuity driven reconstructions showed an improvement in the functional properties. The reduction 
of properties in the non interface engineered samples can be attributed to a larger amount of intermixing in these samples compared to the interface engineered samples. This intermixing is the result of the structural reconstruction of the polar discontinuity.

It is suggested that this method of interface engineering is also interesting for other mixed valence compounds, such as the cuprate superconductors. These compounds generally have a polar discontinuity at the interface with the substrate, possibly affecting film growth, and at the interface with other materials in the required device stack, with a possible reduction of the superconductivity at the interface [49]. The interface engineering is expected to result in improved growth and quality of the thin films and improved properties at the interfaces.

\section{$5.4(110)_{\mathrm{pc}}$ oriented interfaces}

In the literature, several reports discuss the effect of the $(110)_{\mathrm{pc}}$ orientation on the properties of LSMO interfaces. Ma et al. have studied LSMO/STO in superlattice geometry and found a general improvement of the LSMO properties for the $(110)_{\mathrm{pc}}$ orientation [31]. Tebano et al. studied the effect of the crystal orientation in LSMO thin films on $\mathrm{LaAlO}_{3}$ substrates and found better conductivity in the $(110)_{\mathrm{pc}}$ direction [33]. Minohara et al. prepared LSMO/STO Schottky junction devices in the $(001)_{\mathrm{pc}}$ and $(110)_{\mathrm{pc}}$ orientation and observed similar device performance, but an improvement in the magnetization of the $(110)_{\mathrm{pc}}$ oriented films [32]. Also, for $\mathrm{La}_{0.67} \mathrm{Ca}_{0.33} \mathrm{MnO}_{3}$ thin films the magnetization at the interface was improved in the $(110)_{\mathrm{pc}}$ orientation [50].

Note that growth in the $(110)_{\mathrm{pc}}$ also prevents polar discontinuities at the interfaces [51]. In the $(110)_{\mathrm{pc}}$ direction, an $\mathrm{ABO}_{3}$ perovskite has alternating $A B \mathrm{O}$ and $\mathrm{O}_{2}$ layers which have a net charge of 4 and $-4 e-/$ uc respectively. This charge distribution does not depend on the $A$ and $B$ cations and therefore no polar discontinuity is present at the interface between the different materials ${ }^{2}$.

Here, the properties of $(110)_{\mathrm{pc}}$ oriented LSMO/STO interfaces are studied. Ultrathin films with different thicknesses as well as with and without an STO capping layer were grown and characterized. The results show that the electric dead layer of LSMO in the $(110)_{\mathrm{pc}}$ direction is $9 \mathrm{uc}(1 \mathrm{uc}$ corresponds to $\approx 2.7 \AA)$. For the insulating samples, a qualitative difference in the ferromagnetism is observed. No magnetic dead layer is present and the magnetization persists at least up to 350 K.

\subsubsection{Crystal structure}

The crystal structure of the films was determined from X-ray diffraction (XRD) measurements. Reciprocal space maps were obtained around the $(420)_{\mathrm{pc}},(240)_{\mathrm{pc}}$,

\footnotetext{
${ }^{2}$ It is noted that the $(110)_{\mathrm{pc}}$ surface plane is polar and must recontruct. A likely possibility is the creation of $\mathrm{O}$ vacancies in the $\mathrm{O}_{2}$ layer.
} 


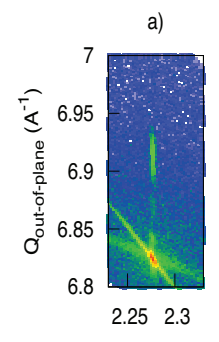

b)

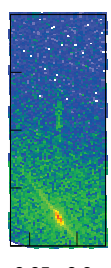

2.252 .3 c)

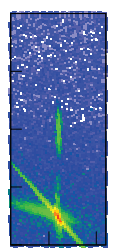

$1.6 \quad 1.65$ d)

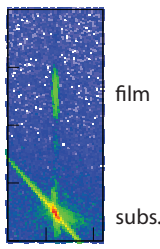

1.61 .65
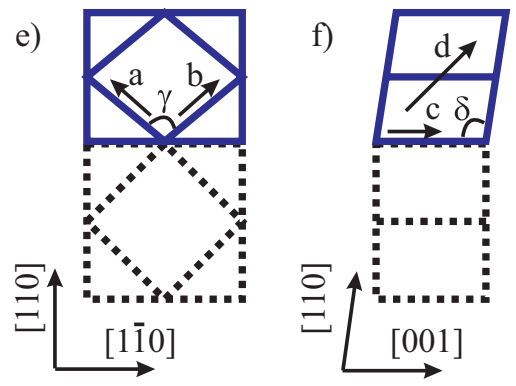

Figure 5.10: Reciprocal space maps around the a) $(420)_{\mathrm{pc}}$, b) $(240)_{\mathrm{pc}}$, c) $(331)_{\mathrm{pc}}$ and d) $(33 \overline{1})_{\mathrm{pc}}$ STO reflections of a $22 \mathrm{~nm}$ thick sample. All film peaks have in-plane momentum transfer equal to that of the substrate. In c) and d) a dissimilar spacing of the (331) and (331) LSMO reflections is clearly observed. This indicates a tilt of the LSMO $(001)_{\mathrm{pc}}$ planes with respect to the $[001]_{\mathrm{pc}}$ lattice vector. A schematic of the crystal structure is shown in e) and f) with the view along the $[001]_{\mathrm{pc}}$ respectively $[1 \overline{1} 0]_{\mathrm{pc}}$ direction. The black dotted line indicates the STO substrate while the blue line is the LSMO unit cell. The $\mathbf{a}, \mathbf{b}$ and $\mathbf{c}$ lattice parameters and the long body diagonal $\mathbf{d}$ are indicated. The angle $\delta$ corresponds to $\angle[(\mathbf{a}+\mathbf{b}), \mathbf{c}]$.

This figure is published identically in [52]. 
Table 5.1: Lattice parameters of LSMO, grown on STO (110)c, as determined from XRD measurements. The in-plane lattice parameters are equal to those of the STO $(110)_{\mathrm{c}}$ surface unit cell. The error in the length is $0.005 \AA$ and the error in the angle is $0.1^{\circ}$.

\begin{tabular}{lccc}
\hline \hline $\begin{array}{l}\text { LSMO film } \\
\text { lattice vectors }\end{array}$ & $\begin{array}{c}\text { Length } \\
(\AA)\end{array}$ & $\begin{array}{c}\text { LSMO } \\
\text { angles }\end{array}$ & $\begin{array}{c}\text { Angle } \\
\left({ }^{\circ}\right)\end{array}$ \\
\hline $\mathbf{a}$ & 3.88 & $\alpha$ & 90.4 \\
$\mathbf{b}$ & 3.88 & $\beta$ & 90.4 \\
$\mathbf{c}=\mathbf{a}_{\text {STO (in-plane) }}$ & 3.905 & $\gamma$ & 90.8 \\
$|(\mathbf{a}+\mathbf{b})|$ (out-of-plane) & 5.45 & $\delta=\angle[(\mathbf{a}+\mathbf{b}), \mathbf{c}]$ & 90.6 \\
$|(\mathbf{a}-\mathbf{b})|=\sqrt{2} \mathbf{a}_{\text {STO }}$ & 5.552 & $\angle[(\mathbf{a}-\mathbf{b}), \mathbf{c}]$ & 90 \\
(in-plane) & & $\angle[(\mathbf{a}-\mathbf{b}),(\mathbf{a}+\mathbf{b})]$ & 90 \\
$\mathbf{d}$ (long body diagonal) & 6.82 & & \\
& & & \\
\hline \hline
\end{tabular}

$(331)_{\mathrm{pc}}$ and $(33 \overline{1})_{\mathrm{pc}}$ STO reflections, as shown in figure 5.10. All maps showed an LSMO peak with in-plane momentum transfer equal to that of the substrate peak, indicating coherent growth and therefore in-plane lattice parameters equal to those of the substrate. Due to the tensile strain the out-of-plane $(110)_{\mathrm{pc}}$ plane spacing of the LSMO is reduced with respect to the bulk value. This results in an angle $\gamma$ between the $\mathbf{a}$ and $\mathbf{b}$ lattice vectors of $90.8^{\circ}$. No significant strain is present on the $\mathbf{a}=\mathbf{b}=3.88 \AA$ lattice parameters themselves, which are equal to the bulk LSMO values [53, 54]. Further, a difference in out-of-plane momentum transfer between the LSMO $(331)_{\mathrm{pc}}$ and $(33 \overline{1})_{\mathrm{pc}}$ reflections is observed. This indicates a tilt of the $(001)_{\mathrm{pc}}$ planes with respect to the plane of the substrate. This difference corresponds to an angle of 90.6 between the LSMO $(001)_{\mathrm{pc}}$ and $(110)_{\mathrm{pc}}$ planes and results in an angle $\alpha=(\beta=) 90.4^{\circ}$ between the $\mathbf{b}(\mathbf{a})$ and $\mathbf{c}$ lattice vector. This corresponds well with the angle between the lattice vectors in bulk rhombohedral LSMO (a cube compressed along the $[111]_{\mathrm{c}}$ direction with $\alpha=\beta=\gamma=90.35^{\circ}$ ). In conclusion, LSMO grown on STO $(110)_{\mathrm{c}}$ has two main deviations from the bulk rhombohedral crystal structure: the c lattice parameter is elongated to match the lattice parameter of the STO and the angle $\gamma$ is increased from $90.35^{\circ}$ to $90.8^{\circ}$. The lattice parameters and angles are summarized in tabel 5.1.

\subsubsection{Functional properties}

At first, the magnetic properties of the $(110)_{\mathrm{pc}}$ oriented samples are discussed. For thick samples, the $T_{\mathrm{C}}$ is $350 \mathrm{~K}$, equal to the $T_{\mathrm{C}}$ of the $(001)_{\mathrm{pc}}$ oriented samples. The temperature dependence of the saturation magnetization of a set of samples with different thicknesses, as well as with and without an 8 uc STO capping layer, is presented in figure 5.11. The $n=10$ samples show the expected magnetic behaviour. The low temperature saturation magnetization is $3.6 \pm 0.1 \mu_{\mathrm{B}} / \mathrm{Mn}$ and the $T_{\mathrm{C}}$ is reduced to $240 \mathrm{~K}$. The samples with $n<10$ show different behaviour. 
At low temperatures, the saturation magnetization is high, even $3.6 \pm 0.2 \mu_{\mathrm{B}} / \mathrm{Mn}$ for the capped $n=3$ sample. This indicates that no magnetic dead layer is present in the $(110)_{\mathrm{pc}}$ orientation. With increasing temperature, the magnetization decreases until it becomes constant at the first critical temperature, $T_{\mathrm{c} 1}$. Above $T_{\mathrm{c} 1}$, the saturation magnetization is $\approx 1.2 \pm 0.2 \mu_{\mathrm{B}} / \mathrm{Mn}$. The samples were clearly ferromagnetic up to $350 \mathrm{~K}$, the maximum operating temperature of the VSM. The uncapped $n=3$ sample, however, was only ferromagnetic up to $40 \mathrm{~K}$.

Magnetic hysteresis loops of the uncapped $n=9$ and $n=10$ samples are presented in figure 5.12. The loops obtained at $T=10 \mathrm{~K}$ have a significant remanent magnetization and a saturation magnetization close to the maximum value of 4 $\mu_{\mathrm{B}} / \mathrm{Mn}$. The coercivity of the $n=9$ sample is very large, $60 \mathrm{kA} / \mathrm{m}$. This indicates strong pinning of the domain walls. This large coercivity was not observed for the other $n<10$ samples, so it can not be attributed to an intrinsic property of the ferromagnetic phase in the $n<10$ samples. The main difference between the two samples is observed in the measurement performed at $T=350 \mathrm{~K}$. The $n=10$ sample does not have a spontaneous magnetization and only a small, 0.2 $\pm 0.1 \mu_{\mathrm{B}} / \mathrm{Mn}$, magnetic signal is observed. In contrast, the $n=9$ sample shows hysteretic behaviour, as shown in figure $5.12 \mathrm{~b}$, with a saturated moment of $1 \pm$ $0.1 \mu_{\mathrm{B}} / \mathrm{Mn}$.

Next, the conductivity of the samples is discussed. The temperature dependent resistivity measurements are presented in figure 5.13. The residual resistivity of the thick $(n \geq 20)$ samples is $200 \mu \Omega \mathrm{cm}$, a factor of three larger than the residual resistivity of $(001)_{\mathrm{pc}}$ oriented films. This difference is attributed to the different crystal structure of the $(110)_{\mathrm{pc}}$ oriented film, which affects the Mn-O-Mn orbital overlap. The conductivity of the $n=10$ and $n=14$ samples is reduced but still measurable at $10 \mathrm{~K}$. Both samples show resistivity curves which indicate a metal insulator transition occurs at $T_{\mathrm{C}}$, which was also verified with magnetoresistance measurements. The samples with $n<10$ were insulating at low temperature and did not show a metal insulator transition.

\subsubsection{Discussion}

The $(110)_{\mathrm{pc}}$ oriented samples can be arranged into two groups. Samples with $n \geq$ 10 are conducting and ferromagnetic, similar to the $(001)_{\mathrm{pc}}$ oriented samples. The samples with $n<10$, however, are insulating and ferromagnetic with two critical temperatures. Above $T_{\mathrm{c} 1}$, the magnetization is almost constant with temperature at $1 \pm 0.1 \mu_{\mathrm{B}} / \mathrm{Mn}$.

In order to explain the ferromagnetic insulating phase, a structural phase transition is assumed. For thick films, the angle $\delta$ is $90.6^{\circ}$, see figure 5.10. It is suggested here that this angle is $90^{\circ}$ for the samples with $n<10$ and that the angular distortion develops with thickness for the samples with $n \geq 10$. In that case, the symmetry changes between an orthorhombic spacegroup for the $n<10$ samples and a monoclinic spacegroup for the $n \geq 10$ samples. The orthorhombic space- 


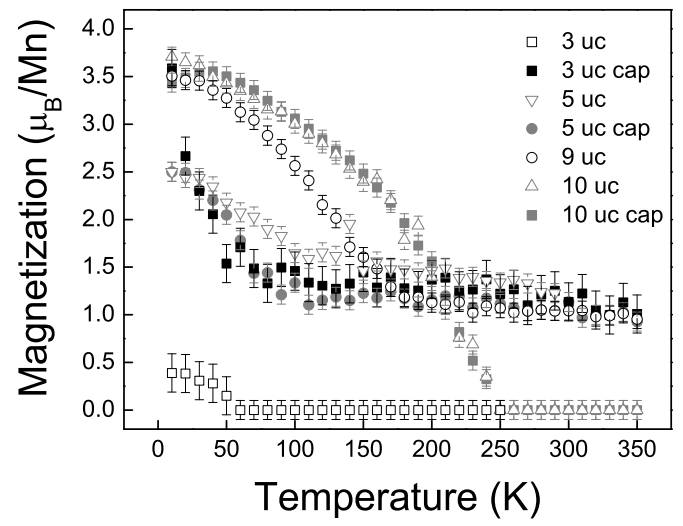

Figure 5.11: Temperature dependent magnetization measurements of $(110)_{\mathrm{pc}}$ oriented films. An $n=8$ capped sample showed qualitatively equal behaviour to the uncapped $n=9$ sample, but the saturation magnetization was not quantatively measured.
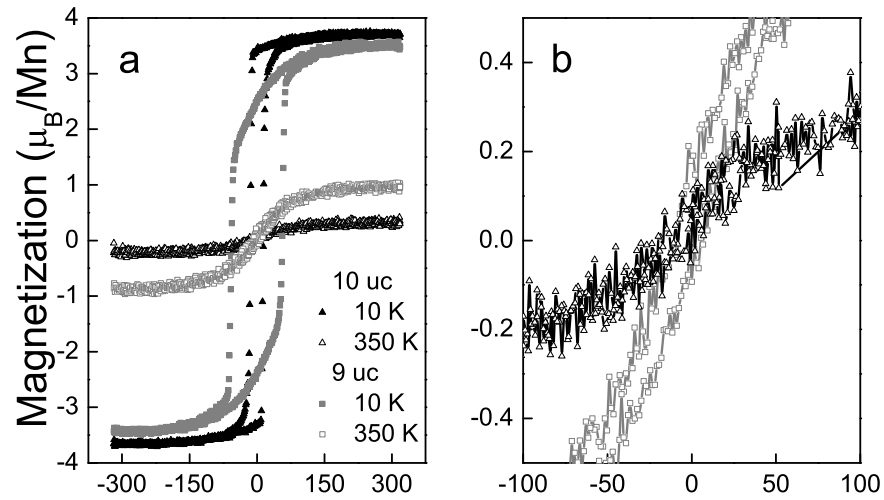

Applied field $(\mathrm{kA} / \mathrm{m})$

Figure 5.12: Magnetization measurements of the $n=9$ and $n=10$ uncapped $(110)_{\mathrm{pc}}$ oriented samples. a) Hysteresis loops at different temperatures.

b) Close-up near the remanent magnetization at $T=350 \mathrm{~K}$. 


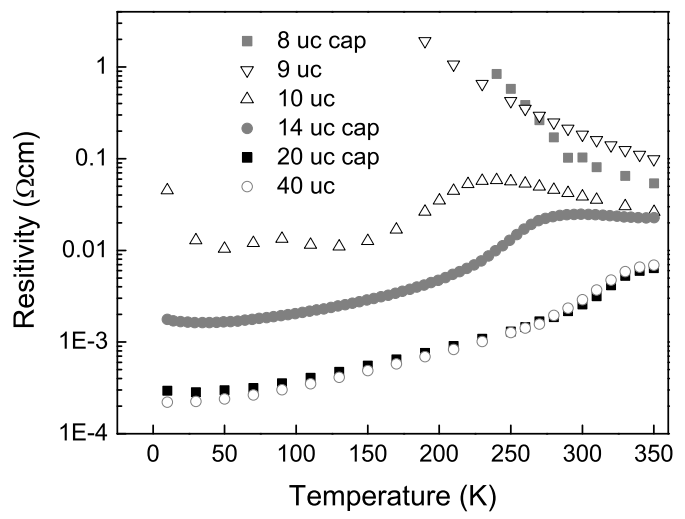

Figure 5.13: Temperature dependent resistivity measurements of $(110)_{\mathrm{pc}}$ oriented films.

group can be described with the Glazer tilt system no. $10,\left(\mathrm{a}^{-} \mathrm{a}^{-} \mathrm{c}^{+}\right)^{3}$, while the monoclinic spacegroup can be described with tilt system no. 13, $\left(\mathrm{a}^{-} \mathrm{a}^{-} \mathrm{c}^{-}\right)$[55]. The latter tilt system is very similar to the tilt system of the bulk rhombohedral structure, no. 14, $\left(\mathrm{a}^{-} \mathrm{a}^{-} \mathrm{a}^{-}\right)$.

In the bulk phase diagram, figure 3.2 , the transition between the ferromagnetic insulating phase and the ferromagnetic metallic phase occurs at $x=0.18$, simultaneously with the orthorhombic/rhombohedral phase transition. In the rhombohedral phase, the Jahn Teller distortion is reduced, resulting in an increase of the bandwidth and conductivity. Therefore, the ferromagnetic insulating phase in the $n<10$ samples can be understood in terms of localization of the $e_{g}$ electrons by Jahn Teller distorted octahedra, enabled by the orthorhombic phase.

It remains to be explained why the $T_{\mathrm{C}}$ of the $n<10$ samples is much larger than the $T_{\mathrm{C}}$ of the bulk ferromagnetic insulating phase. This is attributed to the strain in the layer. An increase of $T_{\mathrm{C}}$ with tensile strain was also reported for $\mathrm{La}_{0.8} \mathrm{Ba}_{0.2} \mathrm{MnO}_{3}$ thin films and for LSMO/BTO superlattices $[48,56]$. Due to the tensile strain, Jahn Teller distorted octahedra result in stronger in-plane ferromagnetic coupling.

\subsubsection{Conclusions}

The $(110)_{\mathrm{pc}}$ oriented samples show similar electrical dead layer behaviour to the $(001)_{\mathrm{pc}}$ oriented samples, although the overall conductivity of the samples was reduced. No evidence for a magnetic dead layer was found. For thin samples, $n<$

\footnotetext{
${ }^{3}$ In the Glazer tilt systems, rotations around the pseudocubic axes are indicate with letters. Identical letters indicate a rotation by an equal amount. The superscripts indicate whether the rotations of two adjacent octahedra are in-phase $\left(^{+}\right)$or out-of-phase $\left(^{-}\right)$.
} 
$10 \mathrm{uc}$, a ferromagnetic insulating phase was found with $T_{\mathrm{C}}$ larger than $350 \mathrm{~K}$. By assuming a structurally different phase for the $n<10$ samples, the ferromagnetic insulating phase can be explained. Finally, it is noted that the ferromagnetic insulating phase in the $n<10$ samples is potentially usefull for spin polarized tunnelling devices [57-59].

\subsection{Order and disorder at the interface}

LSMO is a solid solution of $\mathrm{LaMnO}_{3}$ (LMO) and $\mathrm{SrMnO}_{3}(\mathrm{SMO})$. This implies LSMO is an intrinsically disordered material, where the $A$-site ion on a certain lattice site can be occupied with either a La or a Sr ion. It is an interesting question whether the disorder affects the properties, e.g. electrical transport. Several studies have presented results on the properties of $(\mathrm{LMO})_{2 n} /(\mathrm{SMO})_{n}$ superlattices, which have an identical La/Sr ratio with respect to LSMO [60-62]. A decrease of the residual resistivity, with respect to the solid solution, was observed for the $n=1$ superlattice [62].

Here, the effect of the disorder on the properties at the interface between LSMO and STO is studied. It is proposed that the STO/SMO interface has no intrinsic disorder, as the $A$-site ions of both materials are equal. To obtain LSMO's functional properties at the interface, charge has to be supplied to the SMO layer. This is accomplished by chemically doping the STO away from the interface. This results in modulation doping [63], in which charge is transferred from the doped STO layer to the SMO layer. A schematic band diagram of the STO, La-doped STO, SMO and LSMO is shown in figure 5.14a. La-doped STO's free electrons are indicated with a grey area, just as the electron density in the LSMO. The electrons have high energy with respect to the conduction band of SMO (bandgap $2.3 \mathrm{eV}$ and valence band alignment approximately equal to that of STO), which results in the charge transfer.

The amount of charge which can be transferred is limited by the potential drop due to the electric dipole between the transferred electrons and the positively charged dopants. Eventually, the potential drop increases the energy of the SMO conduction band to the energy of the STO conduction band and then the charge transfer stops. This is shown in figure 5.14b. The maximum charge transfer is then given by:

$$
x=\frac{\Delta \epsilon_{0} K}{d},
$$

in which $x$ is the maximum charge transfer, $\Delta$ is the energy difference between the STO and SMO conduction bands, $\epsilon_{0}$ is the permittivity of free space, $K$ is the dielectric constant and $d$ is the distance over which the charge transfer occurs ${ }^{4}$.

\footnotetext{
${ }^{4}$ For this analysis, a rigid band scenario was used, which is probably not correct for the amount of charge transfer discussed. It is expected that the band structure of SMO changes with doping and becomes similar to that of LSMO and the energy of the conducting electrons decreases. Therefore, the maximum charge transfer, as calculated by equation 5.1 , can be considered as a lower limit.
} 

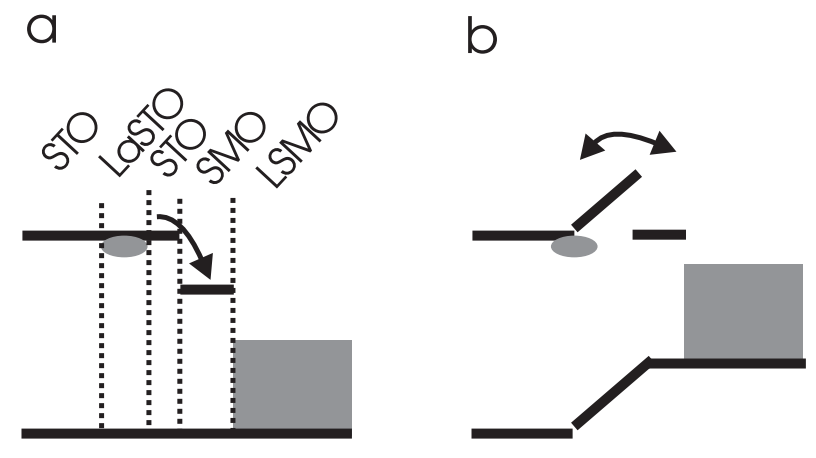

Figure 5.14: A schematic of the band diagram of a modulation doped LSMO interface structure. La-doped STO's free electrons are indicated with a grey area, just as the electron density in the LSMO. a) band diagram with small charge transfer. b) band diagram at the maximum charge transfer.

Therefore, the use of STO has a great advantage; its huge dielectric constant screens the potential drop. It is possible to dope $2 \mathrm{e}$ - $/$ uc over a distance of $2 \mathrm{~nm}$, which is an order of magnitude larger as the charge transfer by modulation doping in semiconductor structures. And at low temperatures, significant charge transfer can be present without significant band bending, as the dielectric constant of STO then becomes 20000 .

\subsubsection{Experimental}

A schematic image of two samples is shown in figure 5.15. Sample A is a reference sample, where an SMO layer is inserted between the LSMO and the STO to obtain the STO/SMO interface. The thickness of the SMO layer is 3 unit cells, which corresponds to the amount of electrically dead layers at the LSMO/STO interface. Next, 1 unit cell layer of $\mathrm{La}_{0.33} \mathrm{Sr}_{0.67} \mathrm{MnO}_{3}$ is present between the $\mathrm{SMO}$ and the LSMO, as this results in higher quality growth of the LSMO, as discussed in section 5.3. The LSMO layer thickness is $6 \mathrm{uc}$, so the sample can be compared with an LSMO sample with a thickness of 10 uc. For this sample no dopants are present in the STO, so the LSMO layer is expected to be overdoped. Sample B is similar to sample A, but here 2 unit cell layers of $\mathrm{LaTiO}_{3}$ (LTO) are inserted in the STO 2 unit cells away from the interface. The LTO layer dopes $2 e-/$ uc to the structure, which is precisely the amount required in the SMO layer to obtain LSMO's $\mathrm{Mn}^{3.33+}$ valence state. Note that only the bottom interface has been engineered to remove the disorder, while the top interface is a disordered LSMO/STO interface.

For the PLD growth of SMO, identical settings were used as for the LSMO growth. For the LTO growth, and the growth of the STO layer on top of the LTO, the deposition pressure was lowered to $3 \cdot 10^{-5} \mathrm{mbar}$, in order to prevent the formation of $\mathrm{La}_{2} \mathrm{Ti}_{2} \mathrm{O}_{7}$ [64]. The electric field gating experiments were performed with a 
a

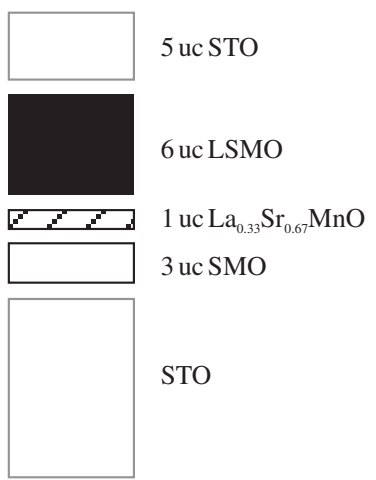

b

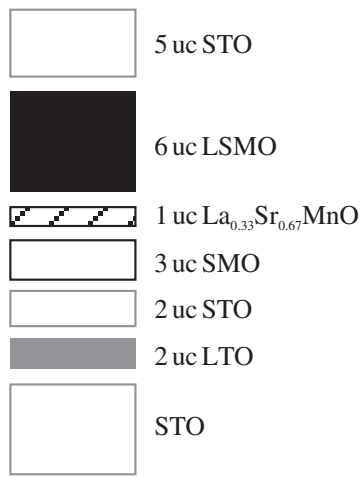

Figure 5.15: A schematic of the sample structure. a) reference sample A.

b) modulation doped sample B.

backgate geometry, using the $0.5 \mathrm{~mm}$ substrate as a gate dielectric. In order to ensure a uniform electric field, the back of the substrate was glued to a copper plate using silver epoxy.

\subsubsection{Results}

The intensity of the specular spot, as monitored with RHEED during the growth of sample B, is shown in figure 5.16. Clear oscillations are observed during the growth of LTO and STO. The initial growth of SMO on the LTO/STO stack started with an increase of the RHEED intensity to a maximum, followed by a prolonged oscillation. This second maximum occurred at the expected time for the deposition of 1.5 unit cell layers. The period of the second oscillation corresponds to the time required to deposit a single unit cell layer. As the SMO deposition was stopped at the third RHEED intensity maximum, only $2.5 \mathrm{uc}$ instead of 3 uc SMO were grown. This initial growth of SMO was not observed for the SMO growth directly on the STO substrate. The inset of figure 5.16a shows the RHEED specular spot intensity observed during the SMO growth of sample A. Here three clear oscillations with equal periods are observed. After the growth of the SMO, the growth of the $\mathrm{La}_{0.33} \mathrm{Sr}_{0.67} \mathrm{MnO}_{3}$, LSMO and STO layers showed RHEED oscillations. Therefore, it is concluded that the entire heterostructure was grown in 2D layer-by-layer fashion.

Electrical transport measurements of the $n=10$ samples are presented in figure 5.17. For comparison, also 2 other samples are shown. Sample C is a 10 unit cell layer LSMO sample capped with 5 unit cell layers of STO. Sample D is similar as sample $\mathrm{C}$, but with engineered interfaces to remove the polar discontinuities, as discussed in section 5.3. The resisitivity of sample B is similar to the values observed for samples $\mathrm{C}$ and $\mathrm{D}$, indicating that sample $\mathrm{B}$ has the properties of a 10 unit cell layer LSMO sample. Sample A, in contrast, has a higher resistivity, lower $T_{\mathrm{C}}$ and negative magnetoresistance present at all temperatures. Therefore, 

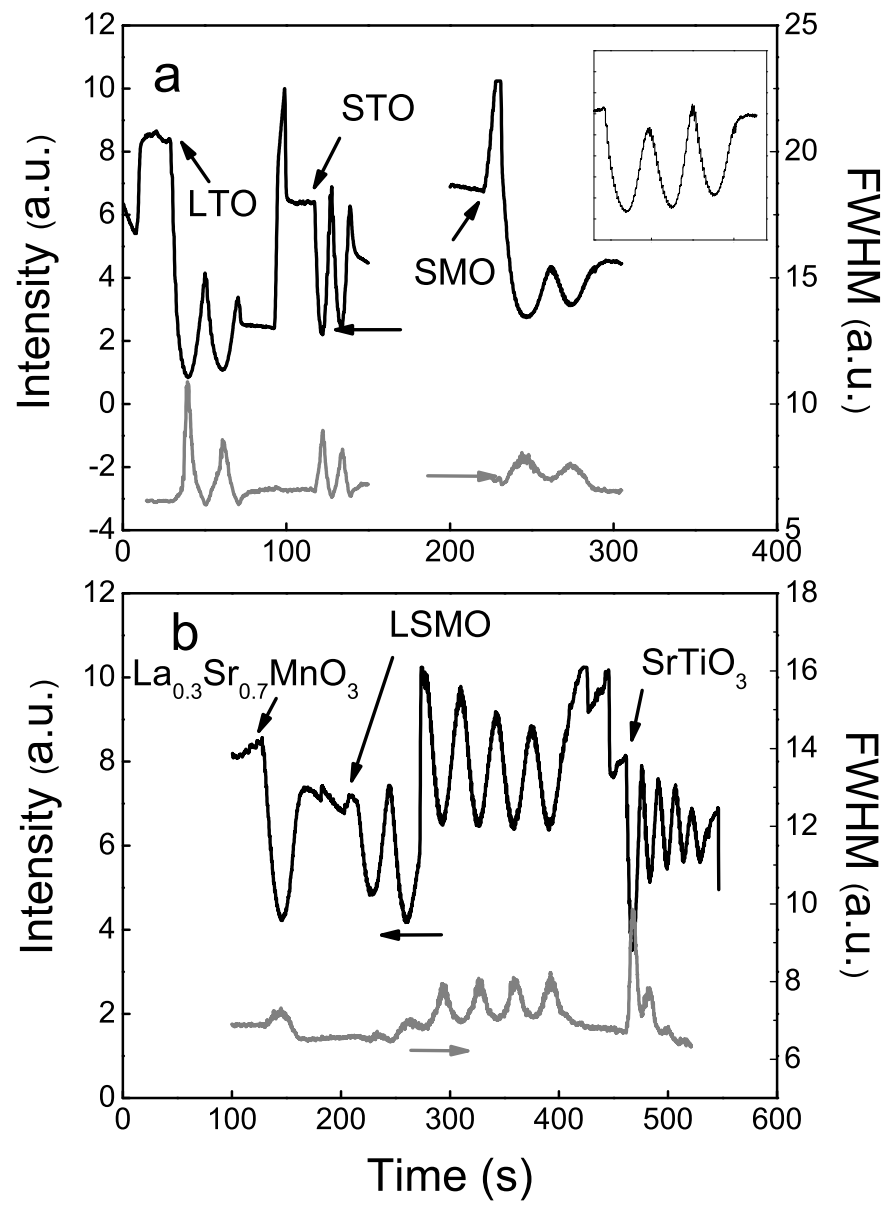

Figure 5.16: RHEED specular spot intensity oscillations and FWHM during the growth of sample B. The starting point of each layer is indicated. The inset in a) shows the RHEED specular spot intensity observed during the growth of SMO directly on the STO substrate, sample A. 

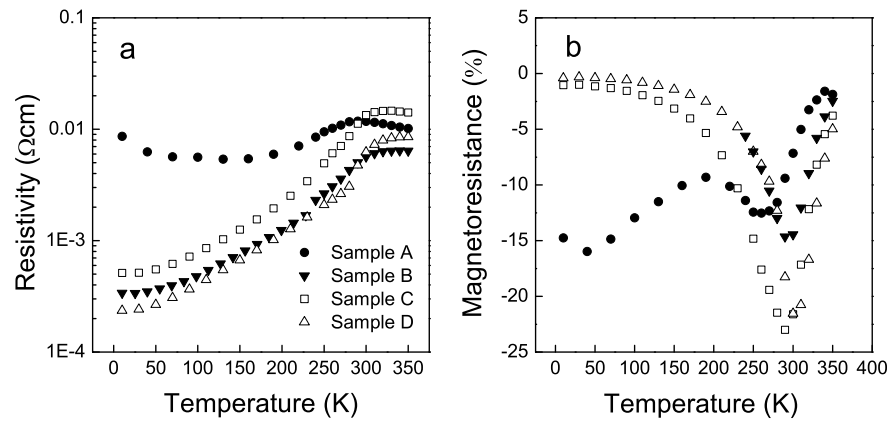

Figure 5.17: Temperature dependent resistivity measurements of four 10 unit cell layer LSMO samples. Sample A contains an SMO layer, but has no modulation doping. Sample B contains the SMO layer and has modulation doping. Sample C is a 10 uc LSMO sample, capped with STO and sample $\mathrm{D}$ is similar to sample $\mathrm{C}$, but with engineered interfaces to remove the polar discontinuity.

sample A can be compared to LSMO samples with a smaller thickness. This result indicates that charge is transferred from the LTO layer to the SMO layer and that the charge transfer significantly changes the conductivity.

In order to verify that the optimum amount of charge is present in the SMO layer, electrical field effect measurements were performed. The charge carrier density of the sample can be controlled with the gate voltage. Figure 5.18 presents the gate voltage dependence of the resistivity, measured at $10 \mathrm{~K}$. Sample A shows some hysteretic behaviour, probably due to charge impurities in the STO substrate, but the general dependence is monotonic with gate voltage. A decrease of the resistivity is observed for positive gate voltage (electron accummulation in the SMO). This corresponds well with the expected overdoping of sample A. For sample B, a minimum in the resistivity is observed at a gate voltage of $50 \mathrm{~V}$. This indicates that the optimum charge carrier density for the conductivity of the LSMO is very close to the doping achieved with the modulation doping. Using an STO dielectric constant of 20000, at most $0.015 \mathrm{e}$-/uc charge carriers are accummulated by the electric field. From a practical point of view, the control over the amount of dopants is $5 \%$ (approximately 1 laser pulse during deposition) of a unit cell layer of LTO, which corresponds to $0.05 \mathrm{e}-/ \mathrm{uc}$. Therefore, it is concluded that sample $\mathrm{B}$ is as good as can presently be realized and no further room for improvement exists in optimizing the inserted amount of dopants.

\subsubsection{Conclusions}

The experiments indicate that modulation doping with a significant charge transfer was achieved. However, LSMO's conductivity at the interface was not improved. 


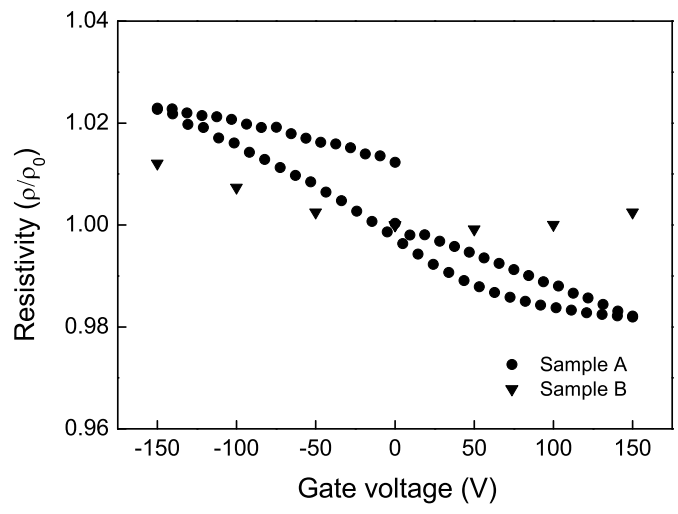

Figure 5.18: The gate voltage dependence of the resistivity.

It is suggested that the large charge transfer achieved here can be very valuable for other oxide 2D materials, such as the recently synthesized tetragonal $\mathrm{CuO}$ [65] and the 2D Mott insulator state in $\mathrm{LaAlO}_{3} / \mathrm{LaTiO}_{3}$ structures [66].

\subsection{Mechanism of the reduction of the properties at LSMO interfaces}

In this chapter, several strategies are described to eliminate the dead layer at the LSMO/STO interface. The reconstructions induced by the polar discontinuity at the interface, the difference between $(001)_{\mathrm{pc}}$ and $(110)_{\mathrm{pc}}$ oriented interfaces and the effect of order versus disorder at the interface were investigated. All modifications made to the interfaces resulted in some improvement in either the magnetization or the conductivity or both. Nevertheless, the dead layer for conductivity remained. In this section, the origin of the dead layer is discussed based on the results obtained in the experiments.

\subsubsection{Overview of the results}

The magnetization and transport measurements of all samples are summarized in figure 5.19, together with the data from $(001)_{\mathrm{pc}}$ oriented LSMO without the STO capping layer obtained from Huijben et al. [38]. In the top graph, the $T_{\mathrm{C}}$ of the samples is shown. The reduction of $T_{\mathrm{C}}$ with decreasing film thickness is approximately the same for all the samples. In the middle graph, the saturation magnetization at $10 \mathrm{~K}$ is shown. Due to the anomalous magnetization in the $(001)_{\mathrm{pc}}$ oriented $n=3$ samples and the ferromagnetic insulating state in the $(110)_{\mathrm{pc}}$ oriented samples, no dead layer can be identified. There is some scatter in 
the data, but overall, the $(110)_{\mathrm{pc}}$ oriented samples have the largest magnetization. Finally, in the bottom graph the residual conductivity of the samples is presented. The conductivity of thick $(110)_{\mathrm{pc}}$ oriented samples (not shown) is a factor of 3 smaller than the conductivity of the $(001)_{\mathrm{pc}}$ oriented samples. For all sets of samples, the conductivity disappears at a critical thickness.

Summarizing the data, the $(001)_{\mathrm{pc}}$ oriented samples without polar discontinuities at the interface have the highest $T_{\mathrm{C}}$ and the largest conductivity. For these samples, the electrical dead layer is $5 \mathrm{uc}$ or $2 \mathrm{~nm}$. The $(001)_{\mathrm{pc}}$ oriented samples capped with STO have a dead layer of $6 \mathrm{uc}$ or $2.4 \mathrm{~nm}$. This is 2 uc thinner compared to the dead layer for the samples without the STO capping. The $(110)_{\mathrm{pc}}$ oriented samples have a dead layer of $9 \mathrm{uc}$ or $2.4 \mathrm{~nm}$, which is similar to the one for $(001)_{\mathrm{pc}}$ oriented LSMO. For these samples, the capping with STO did not change the critical thickness ${ }^{5}$. Finally, the dead layer for the modulation doped sample is unknown, as only one sample was fabricated. From the value for the conductivity of the sample, a critical thickness similar to that of the capped $(001)_{\mathrm{pc}}$ oriented samples is assumed.

\subsubsection{Discussion}

First, the capping with STO is discussed. Several groups have published data which indicates that the functional properties of the $(001)_{\mathrm{pc}}$ oriented LSMO interface deteriorate with the capping by STO $[20,23,24]$. This deterioration was interpreted as an intrinsic property of the LSMO/STO interface. Therefore, the results obtained here, improvement of the conductivity and magnetization with capping, show that this interpretation is not correct. The reduction of functional properties at the LSMO interface originates in the LSMO side of the interface and cannot be attributed to the presence of the STO.

As the dead layer at the interface is still present in all the sets of samples fabricated, the origin of the dead layer cannot be attributed to the polar discontinuity or the disorder at the interface. Three explanations are mentioned in literature, a change in doping at the interface [20-23], an orbital reconstruction [24] and Mn off-centering induced by the oxygen octahedra rotation reconstruction [25]. The first explanation is not applicable to these samples as the local doping was measured with EELS. These measurements demonstrate that the doping at the interface is identical to the doping in the bulk of the film. Furthermore, the orbital reconstruction is also not observed in these samples [38]. It is noted that the orbital reconstruction was only observed in poor quality samples, see section 3.8 , and that capping with STO deteriorated the properties of these samples [24]. Therefore, the orbital reconstruction can be excluded as well. The final mechanism, the oxygen octahedra rotation reconstruction, is then the most likely explanation for the reduction of the properties at the LSMO/STO interface.

\footnotetext{
${ }^{5}$ Note that the ferromagnetic insulating phase for samples with $n<10$ implies that the $n<10$ samples cannot be used to identify the interfacial dead layer for the thicker samples. The conductivity of the $n=10$ sample, however, is already very small, so the critical thickness can be extrapolated from the results of the $n \geq 10$ samples.
} 

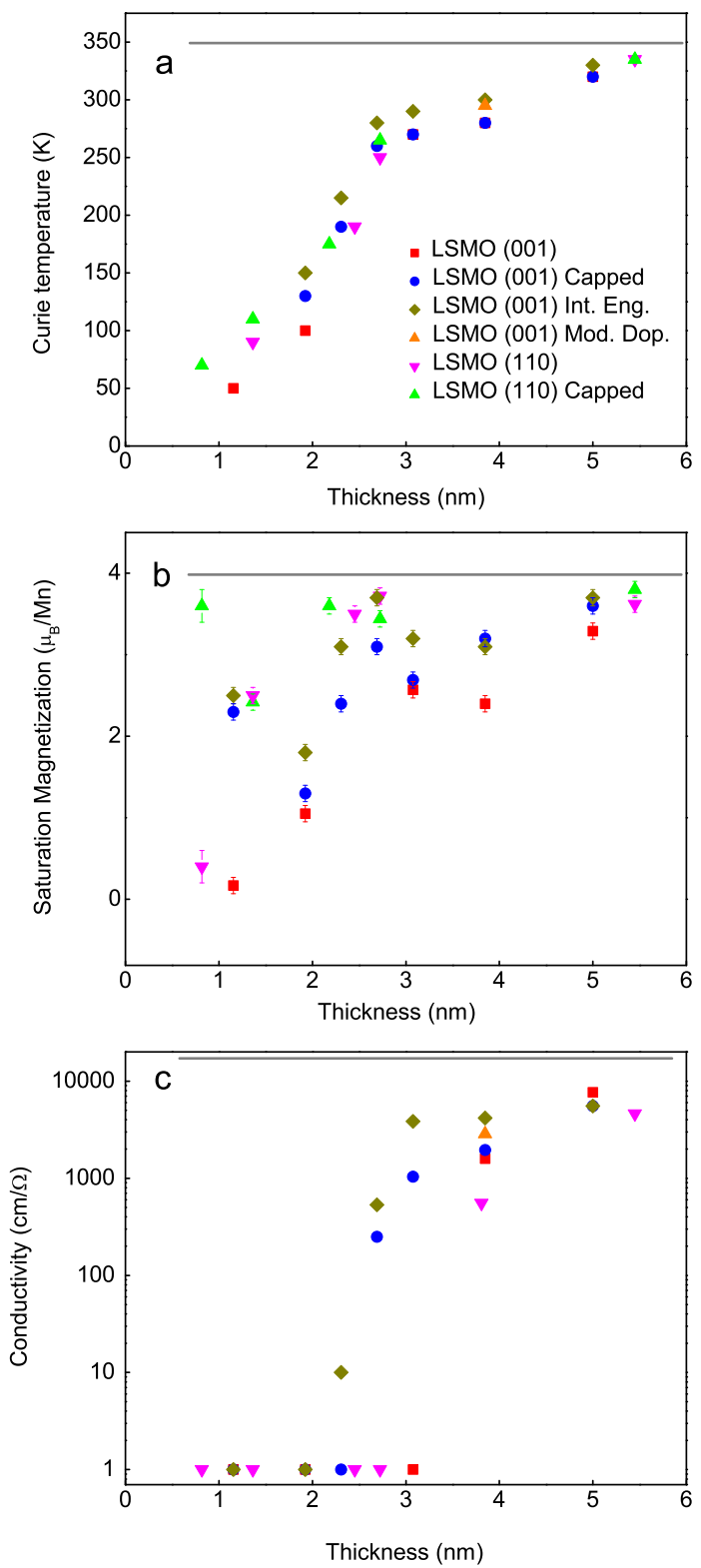

Figure 5.19: LSMO thickness dependence. a) Curie temperature. b) Saturation magnetization at $10 \mathrm{~K}$. c) Conductivity at $10 \mathrm{~K}$. The graphs include data from samples in the $(001)_{\mathrm{pc}}$ and $(110)_{\mathrm{pc}}$ directions, with and without an STO capping layer, samples with engineered interfaces to remove the polar discontinuity (Int. Eng.) and the sample with modulation doping (Mod. Dop.). For the $(110)_{\mathrm{pc}}$ oriented samples with $n<10$, the first critical temperature, as discussed in section 5.4.2, is plotted in a). The conductivity of the insulating samples has been set to 1 . 


\subsubsection{Conclusions}

In this chapter, LSMO/STO interfaces were modified with interface engineering to eliminate the polar discontinuity at the interface, to change the crystal orientation or to eliminate the chemical disorder at the interface. All modifications made to the interfaces resulted in some improvement in either the magnetization or conductivity or both. Nevertheless, the dead layer for conductivity remained. These results indicate that the mechanism proposed by Pruneda et al. [25] is the most likely explanation for the reduction of the properties at the LSMO/STO interface, as the oxygen octahedra rotation pattern at the interface was the only parameter which was not controlled during the experiments. 


\subsection{References}

[1] J. Sun, W. Gallagher, P. Duncombe, L. KrusinElbaum, R. Altman, A. Gupta, Y. Lu, G. Gong, and G. Xiao, "Observation of large low-field magnetoresistance in trilayer perpendicular transport devices made using doped manganate perovskites," APPLIED PHYSICS LETTERS, vol. 69, pp. 3266-3268, 1996.

[2] J. O’Donnell, A. Andrus, S. Oh, E. Colla, and J. Eckstein, "Colossal magnetoresistance magnetic tunnel junctions grown by molecular-beam epitaxy," APPLIED PHYSICS LETTERS, vol. 76, pp. 1914-1916, 2000.

[3] M. Bowen, M. Bibes, A. Barthelemy, J. Contour, A. Anane, Y. Lemaitre, and A. Fert, "Nearly total spin polarization in $\mathrm{La}_{2 / 3} \mathrm{Sr}_{1 / 3} \mathrm{MnO}_{3}$ from tunneling experiments," APPLIED PHYSICS LETTERS, vol. 82, pp. 233-235, 2003.

[4] Y. Ogimoto, M. Izumi, A. Sawa, T. Manako, H. Sato, H. Akoh, M. Kawasaki, and Y. Tokura, "Tunneling magnetoresistance above room temperature in $\mathrm{La}_{0.7} \mathrm{Sr}_{0.3} \mathrm{MnO}_{3} / \mathrm{SrTiO}_{3} / \mathrm{La}_{0.7} \mathrm{Sr}_{0.3} \mathrm{MnO}_{3}$ junctions," JAPANESE JOURNAL OF APPLIED PHYSICS PART 2-LETTERS, vol. 42, pp. L369-L372, 2003.

[5] F. Postma, R. Ramaneti, T. Banerjee, H. Gokcan, E. Haq, D. Blank, R. Jansen, and J. Lodder, "Epitaxial diodes of a half-metallic ferromagnet on an oxide semiconductor," JOURNAL OF APPLIED PHYSICS, vol. 95, pp. 7324-7326, 2004 .

[6] N. Nakagawa, M. Asai, Y. Mukunoki, T. Susaki, and H. Hwang, "Magnetocapacitance and exponential magnetoresistance in manganite-titanate heterojunctions," APPLIED PHYSICS LETTERS, vol. 86, p. xx, 2005.

[7] Y. Hikita, M. Nishikawa, T. Yajima, and H. Y. Hwang, "Termination control of the interface dipole in $\mathrm{La}_{0 .} \mathrm{Sr}_{0.3} \mathrm{MnO}_{3} / \mathrm{Nb}: \mathrm{SrTiO}_{3}$ (001) Schottky junctions," PHYSICAL REVIEW B, vol. 79, p. 073101, 2009.

[8] W. Eerenstein, M. Wiora, J. L. Prieto, J. F. Scott, and N. D. Mathur, "Giant sharp and persistent converse magnetoelectric effects in multiferroic epitaxial heterostructures," NATURE MATERIALS, vol. 6, pp. 348-351, 2007.

[9] H. J. A. Molegraaf, J. Hoffman, C. A. F. Vaz, S. Gariglio, D. van der Marel, C. H. Ahn, and J.-M. Triscone, "Magnetoelectric Effects in Complex Oxides with Competing Ground States," ADVANCED MATERIALS, vol. 21, pp. 3470+, 2009.

[10] V. Garcia, S. Fusil, K. Bouzehouane, S. Enouz-Vedrenne, N. D. Mathur, A. Barthelemy, and M. Bibes, "Giant tunnel electroresistance for non-destructive readout of ferroelectric states," NATURE, vol. 460, pp. 81-84, 2009.

[11] P. Yu, J. S. Lee, S. Okamoto, M. D. Rossell, M. Huijben, C. H. Yang, Q. He, J. X. Zhang, S. Y. Yang, M. J. Lee, Q. M. Ramasse, R. Erni, Y. H. Chu, D. A. Arena, C. C. Kao, L. W. Martin, and R. Ramesh, "Interface Ferromagnetism and Orbital Reconstruction in $\mathrm{BiFeO}_{3}-\mathrm{La}_{0.7} \mathrm{Sr}_{0.3} \mathrm{MnO}_{3}$ Heterostructures," PHYSICAL REVIEW LETTERS, vol. 105, p. 027201, 2010. 
[12] S. M. Wu, S. A. Cybart, P. Yu, M. D. Rossell, J. X. Zhang, R. Ramesh, and R. C. Dynes, "Reversible electric control of exchange bias in a multiferroic field-effect device," NATURE MATERIALS, vol. 9, pp. 756-761, 2010.

[13] A. Y. Borisevich, H. J. Chang, M. Huijben, M. P. Oxley, S. Okamoto, M. K. Niranjan, J. D. Burton, E. Y. Tsymbal, Y. H. Chu, P. Yu, R. Ramesh, S. V. Kalinin, and S. J. Pennycook, "Suppression of Octahedral Tilts and Associated Changes in Electronic Properties at Epitaxial Oxide Heterostructure Interfaces," PHYSICAL REVIEW LETTERS, vol. 105, p. 087204, 2010.

[14] A. Sawa, S. Kashiwaya, H. Obara, H. Yamasaki, M. Koyanagi, N. Yoshida, and Y. Tanaka, "Spin-polarized tunneling of $\mathrm{La}_{0.67} \mathrm{Sr}_{0.33} \mathrm{MnO}_{3} / \mathrm{YBa}_{2} \mathrm{Cu}_{3} \mathrm{O}_{7-\delta}$ junctions," PHYSICA C, vol. 339, pp. 287-297, 2000.

[15] Z. Chen, A. Biswas, I. Zutic, T. Wu, S. Ogale, R. Greene, and T. Venkatesan, "Spin-polarized transport across a $\mathrm{La}_{0.7} \mathrm{Sr}_{0.3} \mathrm{MnO}_{3} / \mathrm{YBa}_{2} \mathrm{Cu}_{3} \mathrm{O}_{7-x}$ interface: Role of Andreev bound states," PHYSICAL REVIEW B, vol. 63, p. 212508, 2001.

[16] V. Pena, Z. Sefrioui, D. Arias, C. Leon, J. Santamaria, J. Martinez, S. te Velthuis, and A. Hoffmann, "Giant magnetoresistance in ferromagnet/superconductor superlattices," PHYSICAL REVIEW LETTERS, vol. 94, p. 057002, 2005.

[17] M. van Zalk, M. Veldhorst, A. Brinkman, J. Aarts, and H. Hilgenkamp, "Magnetization-induced resistance-switching effects in $\mathrm{La}_{0.67} \mathrm{Sr}_{0.33} \mathrm{MnO}_{3} / \mathrm{YBa}_{2} \mathrm{Cu}_{3} \mathrm{O}_{7-\delta}$ bi- and trilayers," PHYSICAL REVIEW B, vol. 79 , p. 134509, 2009.

[18] V. Garcia, M. Bibes, L. Bocher, S. Valencia, F. Kronast, A. Crassous, X. Moya, S. Enouz-Vedrenne, A. Gloter, D. Imhoff, C. Deranlot, N. D. Mathur, S. Fusil, K. Bouzehouane, and A. Barthelemy, "Ferroelectric Control of Spin Polarization," SCIENCE, vol. 327, pp. 1106-1110, 2010 .

[19] C. Barraud, P. Seneor, R. Mattana, S. Fusil, K. Bouzehouane, C. Deranlot, P. Graziosi, L. Hueso, I. Bergenti, V. Dediu, F. Petroff, and A. Fert, "Unravelling the role of the interface for spin injection into organic semiconductors," NATURE PHYSICS, vol. 6, pp. 615-620, 2010.

[20] H. Kumigashira, A. Chikamatsu, R. Hashimoto, M. Oshima, T. Ohnishi, M. Lippmaa, H. Wadati, A. Fujimori, K. Ono, M. Kawasaki, and H. Koinuma, "Robust $\mathrm{Ti}^{4+}$ states in $\mathrm{SrTiO}_{3}$ layers of $\mathrm{La}_{0.6} \mathrm{Sr}_{0.4} \mathrm{MnO}_{3} / \mathrm{SrTiO}_{3} / \mathrm{La}_{0.6} \mathrm{Sr}_{0.4} \mathrm{MnO}_{3}$ junctions," APPLIED PHYSICS LETTERS, vol. 88, p. 192504, 2006.

[21] H. Kumigashira, R. Hashimoto, A. Chikamatsu, M. Oshima, T. Ohnishi, M. Lippmaa, H. Wadati, A. Fujimori, K. Ono, M. Kawasaki, and H. Koinuma, "In situ resonant photoemission characterization of $\mathrm{La}_{0.6} \mathrm{Sr}_{0.4} \mathrm{MnO}_{3}$ layers buried in insulating perovskite oxides," JOURNAL OF APPLIED PHYSICS, vol. 99, p. 08S903, 2006.

[22] J. W. Freeland, J. J. Kavich, K. E. Gray, L. Ozyuzer, H. Zheng, J. F. Mitchell, M. P. Warusawithana, P. Ryan, X. Zhai, R. H. Kodama, and J. N. Eckstein, "Suppressed magnetization at the surfaces and interfaces of ferromagnetic metallic manganites," JOURNAL OF PHYSICS-CONDENSED MATTER, vol. 19, p. 315210, 2007. 
[23] J. J. Kavich, M. P. Warusawithana, J. W. Freeland, P. Ryan, X. Zhai, R. H. Kodama, and J. N. Eckstein, "Nanoscale suppression of magnetization at atomically assembled manganite interfaces: XMCD and XRMS measurements," PHYSICAL REVIEW B, vol. 76, p. 014410, 2007.

[24] A. Tebano, C. Aruta, S. Sanna, P. G. Medaglia, G. Balestrino, A. A. Sidorenko, R. De Renzi, G. Ghiringhelli, L. Braicovich, V. Bisogni, and N. B. Brookes, "Evidence of orbital reconstruction at interfaces in ultrathin $\mathrm{La}_{0.67} \mathrm{Sr}_{0 .}{ }_{33} \mathrm{MnO}_{3}$ films," PHYSICAL REVIEW LETTERS, vol. 100, p. 137401, 2008.

[25] J. M. Pruneda, V. Ferrari, R. Rurali, P. B. Littlewood, N. A. Spaldin, and E. Artacho, "Ferrodistortive instability at the (001) surface of half-metallic manganites," PHYSICAL REVIEW LETTERS, vol. 99, p. 226101, 2007.

[26] R. Herger, P. R. Willmott, C. M. Schlepuetz, M. Bjoerck, S. A. Pauli, D. Martoccia, B. D. Patterson, D. Kumah, R. Clarke, Y. Yacoby, and M. Doebeli, "Structure determination of monolayer-by-monolayer grown $\mathrm{La}_{1-x} \mathrm{Sr}_{x} \mathrm{MnO}_{3}$ thin films and the onset of magnetoresistance," PHYSICAL REVIEW B, vol. 77, p. $085401,2008$.

[27] H. Yamada, Y. Ogawa, Y. Ishii, H. Sato, M. Kawasaki, H. Akoh, and Y. Tokura, "Engineered interface of magnetic oxides," SCIENCE, vol. 305, pp. 646-648, 2004.

[28] Y. Ishii, H. Yamada, H. Sato, H. Akoh, Y. Ogawa, M. Kawasaki, and Y. Tokura, "Improved tunneling magnetoresistance in interface engineered ( $\mathrm{La}, \mathrm{Sr}) \mathrm{MnO}_{3}$ junctions," APPLIED PHYSICS LETTERS, vol. 89, p. 042509, 2006.

[29] A. Ohtomo and H. Hwang, "A high-mobility electron gas at the $\mathrm{LaAlO}_{3} / \mathrm{SrTiO}_{3}$ heterointerface," NATURE, vol. 427, pp. 423-426, 2004.

[30] N. Nakagawa, H. Hwang, and D. Muller, "Why some interfaces cannot be sharp," NATURE MATERIALS, vol. 5, pp. 204-209, 2006.

[31] J. X. Ma, X. F. Liu, T. Lin, G. Y. Gao, J. P. Zhang, W. B. Wu, X. G. Li, and J. Shi, "Interface ferromagnetism in (110)-oriented $\mathrm{La}_{0.7} \mathrm{Sr}_{0.3} \mathrm{MnO}_{3} / \mathrm{SrTiO}_{3}$ ultrathin superlattices," PHYSICAL REVIEW B, vol. 79, p. 174424, 2009.

[32] M. Minohara, Y. Furukawa, R. Yasuhara, H. Kumigashira, and M. Oshima, "Orientation dependence of the Schottky barrier height for $\mathrm{La}_{0.6} \mathrm{Sr}_{0.4} \mathrm{MnO}_{3} / \mathrm{SrTiO}_{3}$ heterojunctions," APPLIED PHYSICS LETTERS, vol. 94 , p. 242106, 2009.

[33] A. Tebano, A. Orsini, D. Di Castro, P. G. Medaglia, and G. Balestrino, "Interplay between crystallographic orientation and electric transport properties in $\mathrm{La}_{2 / 3} \mathrm{Sr}_{1 / 3} \mathrm{MnO}_{3}$ films," APPLIED PHYSICS LETTERS, vol. 96, p. 092505, 2010.

[34] J. Park, E. Vescovo, H. Kim, C. Kwon, R. Ramesh, and T. Venkatesan, "Magnetic properties at surface boundary of a half-metallic ferromagnet $\mathrm{La}_{0.7} \mathrm{Sr}_{0.3} \mathrm{MnO}_{3}$," PHYSICAL REVIEW LETTERS, vol. 81, pp. 1953-1956, 1998.

[35] J. Sun, D. Abraham, R. Rao, and C. Eom, "Thickness-dependent magnetotransport in ultrathin manganite films," APPLIED PHYSICS LETTERS, vol. 74, pp. 3017-3019, 1999. 
[36] R. Borges, W. Guichard, J. Lunney, J. Coey, and F. Ott, "Magnetic and electric "dead" layers in $\left(\mathrm{La}_{0.7} \mathrm{Sr}_{0.3}\right) \mathrm{MnO}_{3}$ thin films," JOURNAL OF APPLIED PHYSICS, vol. 89, pp. 3868-3873, 2001.

[37] M. Angeloni, G. Balestrino, N. Boggio, P. Medaglia, P. Orgiani, and A. Tebano, "Suppression of the metal-insulator transition temperature in thin $\mathrm{La}_{0.7} \mathrm{Sr}_{0.3} \mathrm{MnO}_{3}$ films," JOURNAL OF APPLIED PHYSICS, vol. 96, pp. 6387-6392, 2004.

[38] M. Huijben, L. W. Martin, Y. H. Chu, M. B. Holcomb, P. Yu, G. Rijnders, D. H. A. Blank, and R. Ramesh, "Critical thickness and orbital ordering in ultrathin $\mathrm{La}_{0.7} \mathrm{Sr}_{0.3} \mathrm{MnO}_{3}$ films," PHYSICAL REVIEW B, vol. 78, p. 094413, 2008.

[39] B. Kim, D. Kwon, J. H. Song, Y. Hikita, B. G. Kim, and H. Y. Hwang, "Finite size effect and phase diagram of ultra-thin $\mathrm{La}_{0.7} \mathrm{Sr}_{0.3} \mathrm{MnO}_{3}$," SOLID STATE COMMUNICATIONS, vol. 150, pp. 598-601, 2010.

[40] J. Mannhart, D. H. A. Blank, H. Y. Hwang, A. J. Millis, and J. M. Triscone, "Two-Dimensional Electron Gases at Oxide Interfaces," MRS BULLETIN, vol. 33, pp. 1027-1034, 2008.

[41] C. Lin and A. J. Millis, "Theory of manganite superlattices," PHYSICAL REVIEW B, vol. 78, p. 184405, 2008.

[42] H. Fujishiro, T. Fukase, and M. Ikebe, "Charge ordering and sound velocity anomaly in $\mathrm{La}_{1-x} \mathrm{Sr}_{x} \mathrm{MnO}_{3}(\mathrm{X}>=0.5)$," JOURNAL OF THE PHYSICAL SOCIETY OF JAPAN, vol. 67, pp. 2582-2585, 1998.

[43] G. Koster, B. Kropman, G. Rijnders, D. Blank, and H. Rogalla, "Quasi-ideal strontium titanate crystal surfaces through formation of strontium hydroxide," APPLIED PHYSICS LETTERS, vol. 73, pp. 2920-2922, 1998.

[44] S. Van Aert, J. Verbeeck, R. Erni, S. Bals, M. Luysberg, D. Van Dyck, and G. Van Tendeloo, "Quantitative atomic resolution mapping using high-angle annular dark field scanning transmission electron microscopy," ULTRAMICROSCOPY, vol. 109, pp. 1236-1244, 2009.

[45] H. Tan and J. Verbeeck. private communication, 2010.

[46] L. F. Kourkoutis, J. H. Song, H. Y. Hwang, and D. A. Muller, "Microscopic origins for stabilizing room-temperature ferromagnetism in ultrathin manganite layers," PROCEEDINGS OF THE NATIONAL ACADEMY OF SCIENCES OF THE UNITED STATES OF AMERICA, vol. 107, pp. 11682-11685, 2010.

[47] A. X. Gray, C. Papp, B. Balke, S.-H. Yang, M. Huijben, E. Rotenberg, A. Bostwick, S. Ueda, Y. Yamashita, K. Kobayashi, E. M. Gullikson, J. B.

Kortright, F. M. F. de Groot, G. Rijnders, D. H. A. Blank, R. Ramesh, and C. S. Fadley, "Interface properties of magnetic tunnel junction $\mathrm{La}_{0.7} \mathrm{Sr}_{0.3} \mathrm{MnO}_{3} / \mathrm{SrTiO}_{3}$ superlattices studied by standing-wave excited photoemission spectroscopy," Phys. Rev. B, vol. 82, p. 205116, 2010.

[48] A. Sadoc, B. Mercey, C. Simon, D. Grebille, W. Prellier, and M.-B. Lepetit, "Large Increase of the Curie Temperature by Orbital Ordering Control," PHYSICAL REVIEW LETTERS, vol. 104, p. 046804, 2010. 
[49] G. Koster, A. Brinkman, H. Hilgenkamp, A. J. H. M. Rijnders, and D. H. A. Blank, "High-T-c superconducting thin films with composition control on a sub-unit cell level; the effect of the polar nature of the cuprates," JOURNAL OF PHYSICS-CONDENSED MATTER, vol. 20, p. 264007, 2008.

[50] I. C. Infante, F. Sanchez, J. Fontcuberta, S. Fusil, K. Bouzehouane, G. Herranz, A. Barthelemy, S. Estrade, J. Arbiol, F. Peiro, R. J. O. Mossanek, M. Abbate, and M. Wojcik, "Structural and functional characterization of (110)-oriented epitaxial $\mathrm{La}_{2 / 3} \mathrm{Ca}_{1 / 3} \mathrm{MnO}_{3}$ electrodes and $\mathrm{SrTiO}_{3}$ tunnel barriers," JOURNAL OF APPLIED PHYSICS, vol. 101, p. 093902, 2007.

[51] Y. Mukunoki, N. Nakagawa, T. Susaki, and H. Hwang, "Atomically flat (110) $\mathrm{SrTiO}_{3}$ and heteroepitaxy," APPLIED PHYSICS LETTERS, vol. 86, p. 171908, 2005 .

[52] H. Boschker, J. Kautz, E. P. Houwman, G. Koster, D. H. A. Blank, and G. Rijnders, "Magnetic anisotropy and magnetization reversal of $\mathrm{La}_{0.67} \mathrm{Sr}_{0.33} \mathrm{MnO}_{3}$ thin films on $\mathrm{SrTiO}_{3}(110)$," JOURNAL OF APPLIED PHYSICS, vol. 108, p. 103906, 2010.

[53] A. Hammouche, E. Siebert, and A. Hammou, "Crystallographic, thermal and electrochemical properties of the system $\mathrm{La}_{1-x} \mathrm{Sr}_{x} \mathrm{MnO}_{3}$ for high-temperature solid electrolyte fuel-cells," MATERIALS RESEARCH BULLETIN, vol. 24, pp. 367-380, 1989.

[54] A. Urushibara, Y. Moritomo, T. Arima, A. Asamitsu, G. Kido, and Y. Tokura, "Insulator-metal transition and giant magnetoresistance in $\mathrm{La}_{1-x} \mathrm{Sr}_{x} \mathrm{MnO}_{3}$," Phys. Rev. B, vol. 51, pp. 14103-14109, 1995.

[55] A. M. Glazer, "Classification of tilted octahedra in perovskites," ACTA CRYSTALLOGRAPHICA SECTION B-STRUCTURAL SCIENCE, vol. B 28, p. 3384, 1972.

[56] T. Kanki, H. Tanaka, and T. Kawai, "Anomalous strain effect in $\mathrm{La}_{0.8} \mathrm{Ba}_{0.2} \mathrm{MnO}_{3}$ epitaxial thin film: Role of the orbital degree of freedom in stabilizing ferromagnetism," PHYSICAL REVIEW B, vol. 64, p. 224418, 2001.

[57] J. Moodera, X. Hao, G. Gibson, and R. Meservey, "Electron-spin polarization in tunnel-junctions in zero applied field with ferromagnetic EuS barriers," PHYSICAL REVIEW LETTERS, vol. 61, pp. 637-640, 1988.

[58] D. Worledge and T. Geballe, "Magnetoresistive double spin filter tunnel junction," JOURNAL OF APPLIED PHYSICS, vol. 88, pp. 5277-5279, 2000.

[59] T. Santos and J. Moodera, "Observation of spin filtering with a ferromagnetic EuO tunnel barrier," PHYSICAL REVIEW B, vol. 69, p. 241203, 2004.

[60] H. Yamada, M. Kawasaki, T. Lottermoser, T. Arima, and Y. Tokura, "LaMnO $3 / \mathrm{SrMnO}_{3}$ interfaces with coupled charge-spin-orbital modulation," APPLIED PHYSICS LETTERS, vol. 89, p. 052506, 2006.

[61] C. Adamo, X. Ke, P. Schiffer, A. Soukiassian, M. Warusawithana, L. Maritato, and D. G. Schlom, "Electrical and magnetic properties of $\left(\mathrm{SrMnO}_{3}\right)_{(n)} /\left(\mathrm{LaMnO}_{3}\right)_{(2 n)}$ superlattices," APPLIED PHYSICS LETTERS, vol. 92, p. 112508, 2008. 
[62] A. Bhattacharya, S. J. May, S. G. E. t. Velthuis, M. Warusawithana, X. Zhai, B. Jiang, J. M. Zuo, M. R. Fitzsimmons, S. D. Bader, and J. N. Eckstein, "Metal-insulator transition and its relation to magnetic structure in $\left(\mathrm{LaMnO}_{3}\right)_{(2 n)} /\left(\mathrm{SrMnO}_{3}\right)_{(n)}$ superlattices," PHYSICAL REVIEW LETTERS, vol. 100, p. 257203, 2008.

[63] R. Dingle, H. Stormer, A. Gossard, and W. Wiegmann, "Electron mobilities in modulation-doped semiconductor heterojunction super-lattices," APPLIED PHYSICS LETTERS, vol. 33, pp. 665-667, 1978.

[64] A. Ohtomo, D. Muller, J. Grazul, and H. Hwang, "Epitaxial growth and electronic structure of $\mathrm{LaTiO}_{x}$ films," APPLIED PHYSICS LETTERS, vol. 80, pp. 3922-3924, 2002.

[65] W. Siemons, G. Koster, D. H. A. Blank, R. H. Hammond, T. H. Geballe, and M. R. Beasley, "Tetragonal CuO: End member of the 3d transition metal monoxides," PHYSICAL REVIEW B, vol. 79, p. 195122, 2009.

[66] S. S. A. Seo, M. J. Han, G. W. J. Hassink, W. S. Choi, S. J. Moon, J. S. Kim, T. Susaki, Y. S. Lee, J. Yu, C. Bernhard, H. Y. Hwang, G. Rijnders, D. H. A. Blank, B. Keimer, and T. W. Noh, "Two-Dimensional Confinement of 3d(1) Electrons in $\mathrm{LaTiO}_{3} / \mathrm{LaAlO}_{3}$ Multilayers," PHYSICAL REVIEW LETTERS, vol. 104, p. 036401, 2010. 


\section{Chapter 6}

\section{Epilogue}

In this thesis, several research projects were described in which the properties of perovskite oxide materials were investigated with either strain or interface engineering. Although on both areas progress was made, a complete understanding lacks. It is suggested here that future research should focus on the oxygen sublattice.

The strain engineering of the $\mathrm{La}_{0.67} \mathrm{Sr}_{0.33} \mathrm{MnO}_{3}$ (LSMO) thin films, chapter 4, has resulted in control over the magnetization direction. The main insight is that not only the amount of strain is important, but more specifically, the symmetry of the applied strain is the most important tool in strain engineering. This was exploited by straining the LSMO films along different symmetry directions, which resulted in an independent evaluation of the two unique components of the magnetic anisotropy tensor, the Néel parameters $L_{0}$ and $L^{\prime}$. The control over the symmetry of the applied strain is expected to be useful as well for other materials with anisotropic properties, such as ferroelectric and piezoelectric materials.

Chapter 4 also showed that microscopic understanding of the magnetocrystalline anisotropy can only be obtained if the relation between strain and oxygen octahedra rotations and deformations is understood. This is one of the main challenges in understanding strain engineering in perovskite oxide heteroepitaxy. Structural characterization typically only resolves the positions of the heavier $A$ and $B$ cations, while the positions of the $\mathrm{O}$ anions remain unknown. However, the rotations and deformations of the oxygen octahedra determine the properties of the material. Therefore, a better understanding of the materials properties can only be obtained if the oxygen octahedra rotations and deformations can be predicted and measured as a function of strain.

Some progress has recently been made in this direction. With a combination of $\mathrm{x}$-ray diffraction (XRD) and extended x-ray absorption fine structure (EXAFS) measurements Vailionis et al. showed a common misfit strain accomodation in LSMO and $\mathrm{SrRuO}_{3}$ thin films [1]. From symmetry arguments, the oxygen octahedra rotation pattern was identified and shown to depend on the amount of strain applied to the films. The octahedra rotations can be quantified by comparing the 
intensities from half-order Bragg peaks with the calculated structure factor, as was recently shown by May et al. for $\mathrm{LaNiO}_{3}$ films with different strain states [2]. Finally the ability to directly measure the $\mathrm{O}$ positions with scanning transmission electron microscopy (STEM) measurements is extremely valuable as well, especially for interface reconstructions $[3,4]$. However, the latter has not yet been applied to a systematic study of strain in thin films.

The interface engineering of the $\mathrm{LSMO} \mathrm{SrTiO}_{3}$ (STO) interface, chapter 5 , focussed on the control of the charge and chemical order at the interface. Although improvements in the interfacial properties of the LSMO were realized, the interfacial dead layer remains. The most likely explanation for the dead layer is therefore the one proposed by Pruneda et al. [5]; the change in the magnetic interactions due to the difference in oxygen octahedra rotations at the surface/interface. Therefore, the possibility to engineer the oxygen octahedra rotations at the interface would be a valuable tool for the study of this interface.

Research now focusses on obtaining an understanding of the oxygen octahedra rotations and deformations at interfaces and the induced change in electronic properties. From an experimental point of view, STEM studies provide valuable information. E.g. Jia et al. showed the presence of oxygen octahedra rotation and deformation in the STO at the $\mathrm{LaAlO}_{3} / \mathrm{STO}$ interface [6] and Borisevich et al. showed a change in the oxygen octahedra rotation structure in $\mathrm{BiFeO}_{3}(\mathrm{BFO})$ at the BFO/LSMO interface [7]. Both observations are related to a change in the electronic/magnetic structure at the interface. The importance of oxygen octahedra rotations at interfaces is also recognized in theoretical studies. Bousquet et al. showed that the improper ferroelectricity at the $\mathrm{PbTiO}_{3} / \mathrm{STO}$ interface can be explained with the octahedra rotations at the interface [8]. The symmetry mismatch at an interface between materials with different octahedra rotation structures has to result in a structural reconstruction. He et al. investigated this reconstruction theoretically for both LSMO/STO and SRO/STO interfaces and found a material specific resistance of the octahedra to deformation, which controlled the thickness of the interface layer [9].

The main challenge for materials engineers is not the prediction and measurement of oxygen octahedra rotations and the subsequent changes in electronic and magnetic properties, but the control of the amount and direction of the rotations at the interface. Possible approaches are sandwiching materials between layers with small tolerance factors, changing the $A$ cation size at the interface, similar to bulk experiments in the manganites [10], and combining perovskite oxides with infinite layer compounds.

In conclusion, the oxygen octahedra rotation degree of freedom is one of the unique features of the perovskite oxides. Therefore, the ability to control the rotations at the interface will result in better understanding of materials and emergent functionality. 


\subsection{References}

[1] A. Vailionis, H. Boschker, W. Siemons, E. P. Houwman, G. Rijnders, D. H. A. Blank, and G. Koster, "Misfit strain accommodation in epitaxial $A B \mathrm{O}_{3}$ perovskites: lattice rotations and lattice modulations," arXiv:1009.6018v1.

[2] S. J. May, J. W. Kim, J. M. Rondinelli, E. Karapetrova, N. A. Spaldin, A. Bhattacharya, and P. J. Ryan, "Quantifying octahedral rotations in strained perovskite oxide films," PHYSICAL REVIEW B, vol. 82, p. 014110, 2010.

[3] C. Jia, M. Lentzen, and K. Urban, "Atomic-resolution imaging of oxygen in perovskite ceramics," SCIENCE, vol. 299, pp. 870-873, 2003.

[4] C. Jia and K. Urban, "Atomic-resolution measurement of oxygen concentration in oxide materials," SCIENCE, vol. 303, pp. 2001-2004, 2004.

[5] J. M. Pruneda, V. Ferrari, R. Rurali, P. B. Littlewood, N. A. Spaldin, and E. Artacho, "Ferrodistortive instability at the (001) surface of half-metallic manganites," PHYSICAL REVIEW LETTERS, vol. 99, p. 226101, 2007.

[6] C. L. Jia, S. B. Mi, M. Faley, U. Poppe, J. Schubert, and K. Urban, "Oxygen octahedron reconstruction in the $\mathrm{SrTiO}_{3} / \mathrm{LaAlO}_{3}$ heterointerfaces investigated using aberration-corrected ultrahigh-resolution transmission electron microscopy," PHYSICAL REVIEW B, vol. 79, p. 081405, 2009.

[7] A. Y. Borisevich, H. J. Chang, M. Huijben, M. P. Oxley, S. Okamoto, M. K. Niranjan, J. D. Burton, E. Y. Tsymbal, Y. H. Chu, P. Yu, R. Ramesh, S. V. Kalinin, and S. J. Pennycook, "Suppression of Octahedral Tilts and Associated Changes in Electronic Properties at Epitaxial Oxide Heterostructure Interfaces," PHYSICAL REVIEW LETTERS, vol. 105, p. 087204, 2010.

[8] E. Bousquet, M. Dawber, N. Stucki, C. Lichtensteiger, P. Hermet, S. Gariglio, J.-M. Triscone, and P. Ghosez, "Improper ferroelectricity in perovskite oxide artificial superlattices," NATURE, vol. 452, pp. 732-U4, 2008.

[9] J. He, A. Borisevich, S. V. Kalinin, S. J. Pennycook, and S. T. Pantelides, "Control of Octahedral Tilts and Magnetic Properties of Perovskite Oxide Heterostructures by Substrate Symmetry," PHYSICAL REVIEW LETTERS, vol. 105, p. 227203, 2010.

[10] P. Radaelli, G. Iannone, M. Marezio, H. Hwang, S. Cheong, J. Jorgensen, and D. Argyriou, "Structural effects on the magnetic and transport properties of perovskite $\mathrm{A}_{(1-x)} \mathrm{A}_{(x)} \mathrm{MnO}_{3}(\mathrm{x}=0.25,0.30)$," PHYSICAL REVIEW B, vol. 56, pp. 8265-8276, 1997. 


\section{Appendix A}

\section{Determination of the Curie temperature}

When comparing a set of $\mathrm{La}_{0.67} \mathrm{Sr}_{0.33} \mathrm{MnO}_{3}$ (LSMO) samples, the Curie temperature $\left(T_{\mathrm{C}}\right)$ of the samples is an important figure of merit for the sample quality. Therefore, a reliable method to determine $T_{\mathrm{C}}$ is required. Here, a method based on the analysis of the magnetization loops is proposed.

\section{A.1 Magnetization measurements}

$T_{\mathrm{C}}$ is the temperature above which the spontaneous magnetization disappears. However, the magnetic signal of LSMO does not drop to zero at $T_{\mathrm{C}}$ in most measurements, as typically a small background field is used. This background field is necessary as LSMO's magnetization is very weak with a coercivity which approaches zero around $T_{\mathrm{C}}$. LSMO's spontaneous magnetization will therefore be divided into domains with different orientations and no net magnetization can be observed without the background field. Above $T_{\mathrm{C}}$, LSMO has a very large magnetic susceptibility, as it is possible to induce ferromagnetic exchange coupling with the field. Therefore, a magnetization curve will typically show a tail of magnetic signal above $T_{\mathrm{C}}$.

As a result, the precise transition temperature is open to interpretation. In literature, it is generally not mentioned which criterium is used. E.g., Fitting Kourkoutis et al. presented a magnetization curve from which a $T_{\mathrm{C}}$ of $\approx 298 \mathrm{~K}$ was derived [1]. Inspection of the curve, however, shows that $298 \mathrm{~K}$ is the temperature at which the tail of the magnetization vanishes. Alternatively, Molegraaf et al. presented magnetization curves where the $T_{\mathrm{C}}$ was determined using a linear fit to the slope of the magnetization curve below $T_{\mathrm{C}}[2]$. The intercept of the line with the $T$ axis was defined as $T_{\mathrm{C}}$. Here, a different method is proposed based on the analysis of the temperature dependence of the magnetization loops. 

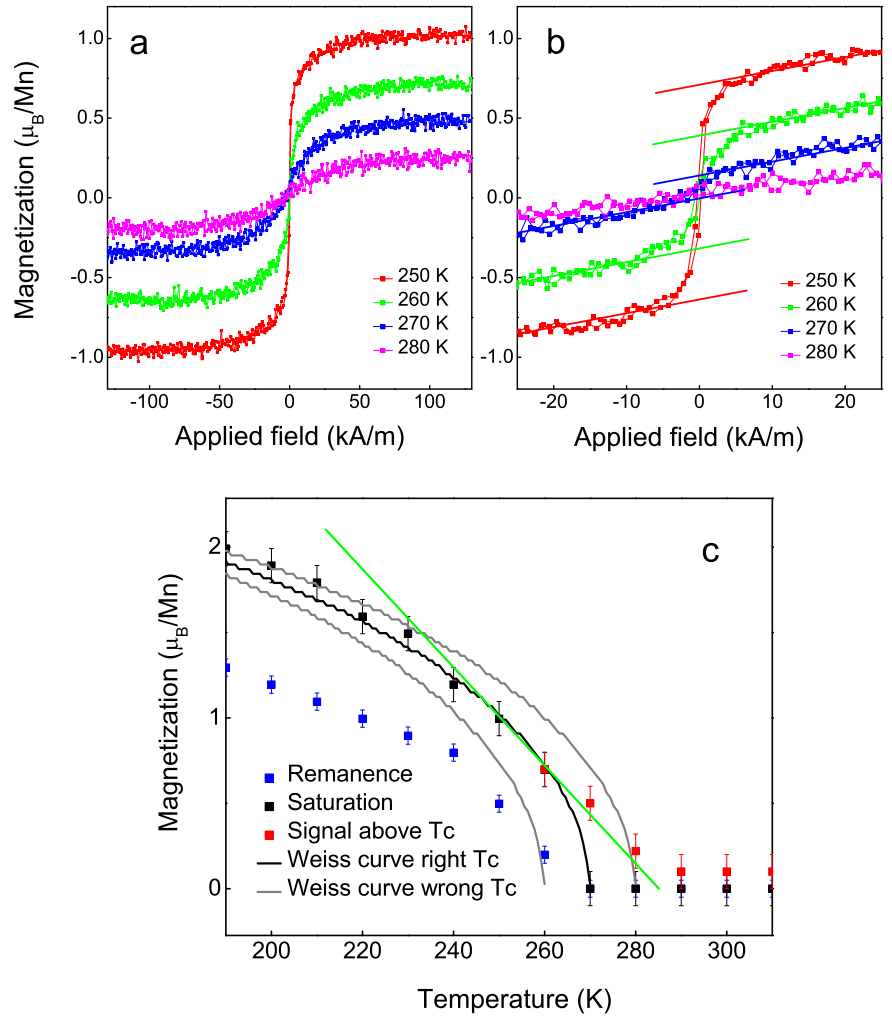

Figure A.1: Magnetization measurements of an 8 unit cell layer LSMO film. The magnetic field was applied parallel to the $[100]_{\mathrm{pc}}$ axis. a) Magnetization loops for different temperatures around $T_{\mathrm{C}}$. b) same as a), but at low applied field. c) Temperature dependence of the magnetization. The remanence plotted is the extrapolated remanent signal, $M_{\text {Rem, extr }}$, as discussed in the main text. The green line is a linear extrapolation of the magnetization curve just below $T_{\mathrm{C}}$. 
In figure A.1a and A.1b, magnetization loops of an 8 unit cell layer LSMO sample for different temperatures close to $T_{\mathrm{C}}$ are presented. All curves show a magnetic saturation with a reduction of the magnetic signal around remanence. A finite coercivity and remanent magnetization are not observed. This is either due to the absence of ferromagnetism (above $T_{\mathrm{C}}$ ) or to domain formation (below $T_{\mathrm{C}}$ ). In order to determine $T_{\mathrm{C}}$, the low field part of the magnetization loops, as shown in figure A.1b, was analyzed. At zero applied magnetic field the magnetization curves at 250 and $260 \mathrm{~K}$ increase rapidly. Then between 10 and $20 \mathrm{kA} / \mathrm{m}$ the curves increase less rapidly and linearly. The rapid increase is attributed to the alignment of domains with different orientations, while the linear increase is attributed to magnetization rotation away from the easy axis to the direction of measurement. The linear part of the magnetization curve can be extrapolated to zero applied field, as shown in figure A.1b. The intercept of the line with the magnetization axis signifies the remanence the sample would have without the domain formation at low applied field, $M_{\text {Rem, extr. }}$. With the definition of $T_{\mathrm{C}}$ as the temperature at which the spontaneous magnetization, and therefore the remanent magnetic signal, disappears, $M_{\text {Rem, extr }}$ can be used for the determination of $T_{\mathrm{C}}$. The criterium for $T_{\mathrm{C}}$ used in this thesis is the lowest temperature at which $M_{\mathrm{Rem}}$, extr $=0$. For the sample shown in figure A.1, $M_{\mathrm{Rem}}$, extr is almost zero at $270 \mathrm{~K}$ and $T_{\mathrm{C}}$ is determined at $270 \mathrm{~K}$.

In figure A.1c, the saturation and $M_{\text {Rem, extr }}$ of the sample are shown. Above $T_{\mathrm{C}}$, the saturation is zero, while the magnetic signal observed in the measurements is indicated as well. The signal above $T_{\mathrm{C}}$ persists at least up to $310 \mathrm{~K}$. The expected temperature dependence of a Weiss ferromagnet, as discussed in section 3.5, is shown as well. Three curves are drawn with a $T_{\mathrm{C}}$ of 260,270 and $280 \mathrm{~K}$ respectively. Only the curve with a $T_{\mathrm{C}}$ of $270 \mathrm{~K}$ fits the data. A linear fit to the magnetization curve below $T_{\mathrm{C}}$ is indicated as well. The intercept of this line with the $T$ axis is $285 \mathrm{~K}$. So, the determination of $T_{\mathrm{C}}$ with the method outlined here results in a lower value for $T_{\mathrm{C}}$ with respect to the reports in literature.

\section{A.2 Transport measurements}

In figure A.2, the transport measurements of the sample are presented. LSMO has a metal insulator transition at $T_{\mathrm{C}}$ and this transition temperature can in principle be obtained from the temperature dependence of the resistivity. The first criterium which is used is the temperature at which the resistivity is maximum [3,4], which corresponds to $310 \mathrm{~K}$ for this sample. The second criterium is the temperature at which the derivative of the resistivity is maximum, $260 \mathrm{~K}$ for this sample. Finally, the magnetoresistance is expected to be maximal at $T_{\mathrm{C}}[5]$. The magnetoresistance is shown in figure A.2b and the maximum occurs at $270 \mathrm{~K}$, which corresponds well with the $T_{\mathrm{C}}$ determined from the magnetization curves. It was found that the maximum in magnetoresistance corresponded with the $T_{\mathrm{C}}$ determined from the magnetization loops for all samples measured. 

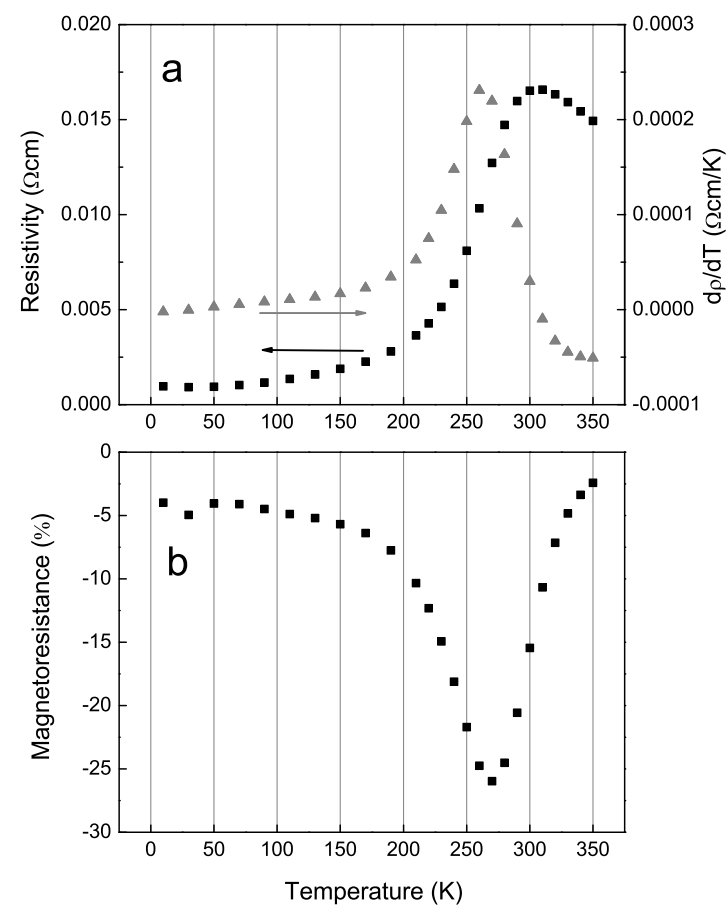

Figure A.2: Transport measurements of an 8 unit cell layer LSMO film. a) Resistivity and its derivative. b) Magnetoresistance $((\rho(2.5 T)-$ $\rho(0)) / \rho(0))$. 


\section{A.3 Conclusion}

A method to determine the $T_{\mathrm{C}}$ of LSMO samples, based on the analysis of the magnetization loops, was proposed. The criterium for $T_{\mathrm{C}}$ is the lowest temperature at which $M_{\text {Rem, extr }}=0$. This criterium results in a $T_{\mathrm{C}}$ which corresponds well with the analysis of the temperature dependence of the magnetization in terms of the Weiss model and it corresponds with the temperature at which the magnetoresistance is maximum. This method for the determination of the $T_{\mathrm{C}}$ typically results in a lower $T_{\mathrm{C}}$ than the methods used in literature, so therefore some care must be taken when comparing samples in this thesis with reports in literature. 


\section{A.4 References}

[1] L. F. Kourkoutis, J. H. Song, H. Y. Hwang, and D. A. Muller, "Microscopic origins for stabilizing room-temperature ferromagnetism in ultrathin manganite layers," PROCEEDINGS OF THE NATIONAL ACADEMY OF SCIENCES OF THE UNITED STATES OF AMERICA, vol. 107, pp. 11682-11685, 2010.

[2] H. J. A. Molegraaf, J. Hoffman, C. A. F. Vaz, S. Gariglio, D. van der Marel, C. H. Ahn, and J.-M. Triscone, "Magnetoelectric Effects in Complex Oxides with Competing Ground States," ADVANCED MATERIALS, vol. 21, pp. 3470+, 2009.

[3] A. Tebano, C. Aruta, P. G. Medaglia, F. Tozzi, G. Balestrino, A. A. Sidorenko, G. Allodi, R. De Renzi, G. Ghiringhelli, C. Dallera, L. Braicovich, and N. B. Brookes, "Strain-induced phase separation in $\mathrm{La}_{0.7} \mathrm{Sr}_{0.3} \mathrm{MnO}_{3}$ thin films," PHYSICAL REVIEW B, vol. 74, p. 245116, 2006.

[4] A. Tebano, C. Aruta, S. Sanna, P. G. Medaglia, G. Balestrino, A. A. Sidorenko, R. De Renzi, G. Ghiringhelli, L. Braicovich, V. Bisogni, and N. B. Brookes, "Evidence of orbital reconstruction at interfaces in ultrathin $\mathrm{La}_{0.67} \mathrm{Sr}_{0 .}{ }_{33} \mathrm{MnO}_{3}$ films," PHYSICAL REVIEW LETTERS, vol. 100, p. 137401, 2008.

[5] P. Schiffer, A. Ramirez, W. Bao, and S. Cheong, "Low-temperature magnetoresistance and the magnetic phase-diagram of $\mathrm{La}_{1-x} \mathrm{Ca}_{x} \mathrm{MnO}_{3}$," PHYSICAL REVIEW LETTERS, vol. 75, pp. 3336-3339, 1995. 


\section{Summary}

Perovskite oxides are an extremely interesting class of materials. The perovskite $A B \mathrm{O}_{3}$ crystal structure can be realized with a wide variety of different elements at the $A$ and $B$ position. This results in a class of materials with similar lattice parameters but a wide range in properties, from dielectrics and piezoelectrics to ferroelectrics, from semiconductors, transparant conductors and metals to superconductors and from antiferromagnetic to ferromagnetic and multiferroic materials. Perovskite oxides are therefore naturally suitable for heteroepitaxy. Perovskite oxide heteroepitaxy is nowadays a large field of research and significant advances have been made to realize even more functionality from the perovskite building blocks.

The new functionality of the heterostructures can be attributed to two fundamental effects in heteroepitaxy. At first the crystal structure of the layers is changed, due to the matching of the in-plane lattice constants to those of the substrate. The matching results in strain in the layers, the magnitude of which can be controlled with the use of an appropriate substrate. This is called strain engineering. Furthermore, the interfaces between different layers break symmetry and therefore new functionality can be expected at the interfaces. Heteroepitaxy allows for direct intervention, e.g. with dopant insertion, at the interface during growth, which is called interface engineering. In this thesis, an exploration of the possibilities with strain and interface engineering is made for both the $\mathrm{LaAlO}_{3} / \mathrm{SrTiO}_{3}(\mathrm{LAO} / \mathrm{STO})$ interface and the fully spin polarized metal $\mathrm{La}_{0.67} \mathrm{Sr}_{0.33} \mathrm{MnO}_{3}$ (LSMO).

At first, the conducting interface between the two band insulators LAO and STO is studied. The main experimental result is that the expected electrostatic potential buildup in the LAO layer is not observed. Furthermore, samples grown on other substrates than STO and samples grown at high oxygen pressure are insulating. The results indicate that the most widely used model to explain the conductivity at the interface, the electronic reconstruction due to the polar discontinuity, is not applicable to the experiments. An alternative model for the conductivity is proposed, in which the polar discontinuity is compensated by structural reconstructions, details of which depend on the growth conditions. At certain growth conditions, the structural reconstructions result in charge transfer to the STO and the charge in the STO resides in impurity states within the bandgap. Conductivity occurs when the induced polarization in the STO activates the charge carriers to the conduction band. 
The largest part of the thesis is concerned with LSMO. High quality LSMO films were fabricated, as demonstrated by thorough characterization of the film growth and properties. The films were grown by pulsed laser deposition, monitored with in situ reflection high energy electron diffraction. The initial film growth occurred in a predominantly layer-by-layer fashion which changed to the steady state growth mode during deposition, resulting in a smooth surface with islands with unit cell step height. Structural characterization showed that the LSMO films were grown in a fully coherent fashion with respect to the underlying substrates. The characterization of the magnetic and electric transport properties of the films showed a saturation magnetization of $4.0 \mu_{\mathrm{B}} / \mathrm{Mn}$, a Curie temperature of $350 \mathrm{~K}$ and a residual resistivity of $70 \mu \Omega \mathrm{cm}$. X-ray photoelectron spectroscopy measurements showed an absence of chemical impurities and no evidence for surface segregation. Scanning transmission electron microscopy showed that multilayer structures with sharp interfaces were realized. All results indicate that high quality films, comparable to the best examples found in literature, were fabricated.

In LSMO thin films, the crystal structure is determined by the strain from the underlying substrate. With the choice of a specific substrate surface, the magnetic properties of the LSMO layer can be controlled. A model, based on the framework of Néel, was developed which predicts the magnetocrystalline anisotropy. The model was verified with measurements of the magnetic anisotropy of LSMO thin films grown on different surfaces of $\mathrm{NdGaO}_{3}$ single crystal substrates. The experiments uniquely demonstrate that both the Néel parameters $L_{0}$ and $L^{\prime}$ are negative. The origin of the anisotropy is the alignment of the Mn orbitals in the LSMO crystal field. The physical meaning of a negative $L_{0}$ is that the shape of the orbitals is oblate. The crystal field due to the neighbouring oxygen ions was evaluated with respect to strain. As $L^{\prime}$ was found to be negative as well, the analysis of the crystal field interaction suggests that the angles of the $\mathrm{MnO}_{6}$ octahedra change with strain while the Mn-O bond lengths remain approximately constant.

The use of LSMO in spintronic devices requires spin polarized conductivity at the interface. Due to interface reconstructions, generally the spin polarized conductivity is reduced or completely absent at the interface. Interface engineering can therefore be a valuable tool to study and improve the interfacial properties. Here, three strategies for the optimization of the LSMO at the interface are presented. The first one is based on analysis of the interface in terms of the recently developed polar discontinuity model for oxide interfaces. Based on the model, the insertion of a single atomic layer of $\mathrm{La}_{0.33} \mathrm{Sr}_{0.67} \mathrm{O}$ is proposed and experimentally realized. The results show improved properties, due to a significant reduction of interdiffusion at the engineered interface. An alternative approach is to study interfaces in the (011) crystal direction. It is shown that (011) LSMO has different properties in comparison to (001) oriented LSMO, with reduced conductivity, but enhanced magnetization. Finally, the effect of chemical order and disorder in the system was studied. To obtain a chemically ordered LSMO interface, a layer of $\mathrm{SrMnO}_{3}$ was inserted, which was doped with the use of modulation doping from donor states in the STO. Preliminary results show that the modulation doping is succesfull, although the effect of the chemical order on the magnetization and conductivity at the interface was small. All three modifications did not result in the desired 
fully spin polarized conductivity at the interfaces. Therefore, it is concluded that the reduction of the properties at the interface is due to the discontinuity in the oxygen octahedra rotations at the interface, as this is the only variable which was not controlled during the experiments.

In conclusion, several research projects were described in which the properties of perovskite oxide materials were investigated with either strain or interface engineering. Although on both areas progress was made, a complete understanding lacks. It is suggested that future research should focus on the oxygen sublattice, especially at the interfaces. The main challenge for materials engineers is not only the prediction and measurement of oxygen octahedra rotations and the subsequent changes in electronic and magnetic properties, but the control of the amount and direction of the rotations at the interface. The oxygen octahedra rotation degree of freedom is one of the unique features of the perovskite oxides. Therefore, the ability to control the rotations at the interface will result in better understanding of materials and emergent functionality. 


\section{Samenvatting (Summary in Dutch)}

De perovskiet oxiden zijn een interessante klasse van materialen. De perovskiet $A B \mathrm{O}_{3}$ kristalstructuur kan worden ingevuld met een groot scala aan verschillende elementen op zowel de $A$ als $B$ positie. Dit resulteert in een klasse van materialen met vergelijkbare kristal parameters en met een groot bereik in eigenschappen, van diëlectrische en piëzo-electrische tot ferro-electrische materialen, van halfgeleiders, transparante geleiders en metalen tot supergeleiders en van anti-ferromagnetische tot ferromagnetische en multi-ferroische materialen. Daarom zijn perovskiet oxiden erg geschikt voor hetero-epitaxie. Dit is tegenwoordig een uitgebreid onderzoeksgebied en er is significante vooruitgang geboekt in het realizeren van nog meer functionaliteit van de perovskiet bouwstenen.

De nieuwe functionaliteit van de hetero-structuren is het gevolg van twee fundamentele aspecten van hetero-epitaxie. Ten eerste wordt de kristalstructuur van de lagen veranderd, door de aanpassing van de rooster parameters aan die van het substraat. Deze aanpassing zorgt voor spanning in de lagen en de hoeveelheid spanning kan worden ingesteld met de keuze van een geschikt substraat. Daarnaast breken de grensvlakken tussen de lagen de kristalsymmetrie en dit geeft ook nieuwe eigenschappen. Met hetero-epitaxie kan men direct ingrijpen op het grensvlak tijdens de groei, bijvoorbeeld door specifieke atomen toe te voegen. In dit proefschrift worden twee materiaal systemen, het grensvlak tussen $\mathrm{LaAlO}_{3}(\mathrm{LAO})$ en $\mathrm{SrTiO}_{3}$ (STO) en het spin-gepolarizeerde metaal $\mathrm{La}_{0.67} \mathrm{Sr}_{0.33} \mathrm{MnO}_{3}$ (LSMO), onderzocht met de technieken van de hetero-epitaxie.

Als eerste is het geleidende grensvlak tussen de isolatoren LAO en STO bestudeerd. Het belangrijkste resultaat is dat de verwachte opbouw van de elektrostatische potentiaal in de LAO laag niet is waargenomen. Ook zijn structuren die gemaakt zijn op een ander substraat dan STO en structuren die gegroeid zijn onder een hoge zuurstofdruk niet geleidend. Deze resultaten tonen aan dat het algemeen gebruikte model om de geleidende eigenschappen van het grensvlak te verklaren, de elektronische reconstructie van de polaire discontinuïteit, niet van toepassing is op de experimenten. Een alternatief model wordt voorgesteld, waarin de polaire discontinuïteit wordt gecompenseerd door middel van structurele reconstructies. Dit resulteert in ladingsoverdracht naar het STO en de lading bevindt zich dan in 
defekt toestanden in de bandkloof. Geleiding treedt op wanneer de geïnduceerde polarizatie in het STO de ladingsdragers activeert naar de geleidingsband.

Het grootste gedeelte van het proefschrift behandelt experimenten met LSMO. Er zijn LSMO films van hoge kwaliteit gemaakt, wat is aangetoond met uitgebreid onderzoek naar de groei en de eigenschappen. De films zijn gemaakt met behulp van gepulste laser depositie en laten een saturatie magnetizatie van $4.0 \mu_{\mathrm{B}} / \mathrm{Mn}$, een Curie temperatuur van $350 \mathrm{~K}$ en een soortelijke weerstand van $70 \mu \Omega \mathrm{cm}$ op $10 \mathrm{~K}$ zien. Deze resultaten zijn goed in vergelijking tot de films beschreven in de literatuur.

De kristalstructuur van de films wordt bepaald door de spanning die wordt opgelegd door het substraat. De magnetische eigenschappen kunnen worden gestuurd met de keuze van een geschikt substraat oppervlak. Er is een model, gebaseerd op het raamwerk van Néel, ontwikkeld dat de magnetisch kristallijne anisotropie van de films beschrijft. Het model is getest met anisotropie metingen op LSMO films, gegroeid op verschillende $\mathrm{NdGaO}_{3}$ substraat oppervlakken. De experimenten tonen aan dat de Néel parameters $L_{0}$ en $L^{\prime}$ beiden negatief zijn. Dit impliceert dat de spanning in de film resulteert in verandering van de hoeken in de zuurstofoctaeders en niet in een verandering in de $\mathrm{Mn}-\mathrm{O}$ afstanden.

De spin-gepolarizeerde geleiding van LSMO aan het grensvlak is doorslaggevend voor de toepassing van LSMO in spin-elektronica. Over het algemeen is de spingepolarizeerde geleiding aan het grensvlak echter verminderd door grensvlakreconstructies. Drie strategieën om dit te verbeteren zijn in dit proefschrift uitgewerkt. Ten eerste is de invloed van de polaire discontinuïteit aan het grensvlak onderzocht. Deze kan worden voorkomen door een enkele $\mathrm{La}_{0.33} \mathrm{Sr}_{0.67} \mathrm{O}$ laag aan de structuur toe te voegen. Films met deze extra laag hebben betere eigenschappen, doordat ongewenste diffusie van atomen aan het grensvlak wordt voorkomen. Verder zijn grensvlakken in de (110) kristalrichting onderzocht. LSMO heeft andere eigenschappen in de (110) richting, met een kleinere geleiding en betere magnetizatie. Ten slotte is de invloed van chemische wanorde onderzocht. Er is een chemisch geordend grensvlak gemaakt door een laag $\mathrm{SrMnO}_{3}$ aan de structuur toe te voegen. De juiste hoeveelheid elektronen in de laag is vervolgens bereikt met modulatie doping vanuit donor toestanden in het STO. De eerste resultaten laten zien dat de modulatie doping succesvol was, maar dat de invloed van de chemische orde klein is. Alle strategieën hebben niet tot de gewenste spin-gepolarizeerde geleiding aan het grensvlak geleid. Daarom is de conclusie van het onderzoek dat de vermindering van de eigenschappen van LSMO grensvlakken te wijten is aan een reconstructie van de zuurstof octaeder structuur aan het grensvlak, de enige parameter die niet onderzocht is.

Samengevat, verschillende onderzoeksprojecten worden beschreven waarin de eigenschappen van perovskiet oxidische materialen worden onderzocht door middel van hetero-epitaxie. Ook al is er enige vooruitgang geboekt, een volledig begrip van de materiaal systemen ontbreekt nog. Er wordt gesuggereerd dat toekomstig onderzoek zich moet richten op het begrijpen van de zuurstof octaeder structuur. Vooral de mogelijkheid deze structuur aan een grensvlak te kunnen aanpassen zal extreem waardevol zijn voor zowel het begrip alsmede de toepassing van de materialen. 


\section{Dankwoord}

$\mathrm{Na}$ vier jaar onderzoek kan ik veel mensen bedanken. Zeker gezien het feit dat ik veel en graag geprofiteerd heb van het werk van anderen. Daarom begin ik met de mensen die hebben bijgedragen aan het onderzoek in dit proefschrift. Ere wie ere toekomt. Hoofdstuk 2 was nooit van de grond gekomen zonder het werk van Luuk, Andreas, Josée en Gerwin. Hoofdstuk 3 is gebaseerd op de goede optimalizatie van de groei van LSMO met PLD, welke gedaan is door Gabriella en Mark. In hoofdstuk 4 hebben Mercy en Hiroaki een grote bijdrage geleverd aan de experimentele resultaten en heeft Evert een essentiële bijdrage geleverd aan de analyse met het Néel model. Vervolgens zijn de experimenten aan de (110) georiënteerde grensvlakken in hoofdstuk 5 gedaan door Jaap. Ook hebben verschillende andere labs experimenten gedaan met mijn samples. Arturas met XRD en Martin, Jo, Sara, Martina, Sandra en Johan met STEM. Dus allemaal heel erg bedankt, zonder jullie was dit boekje erg dun gebleven.

Daarnaast heb ik natuurlijk ook veel begeleiding gehad. In de eerste plaats door Guus. Guus, ik ben erg blij dat je me van het begin af aan de mogelijkheid hebt gegeven om zelf de riching van het onderzoek te bepalen. Veel succes verder als grote baas van IMS. Dan is er de vorige grote baas, Dave. Bedankt, ook voor de etentjes en het introduceren van Górecki 3. Met name bij al het schrijfwerk was de inbreng van Gertjan, Mark en Evert erg nuttig. Alexander, ik heb onze discussies door de jaren heen ook altijd zeer gewaardeerd. Ook de praktische ondersteuning van Henk, Gerrit, Frank, Dick, José en natuurlijk Marion verdient enige aandacht. Bedankt allemaal.

It was a great pleasure to spend a part of my PHD in Tokyo. Harold, thanks for giving me the opportunity to visit your group and for your inspiration. Jajimasan, thanks for all the patience in the lab, it was nice working with you. And Hikita-san, Chris, Tanaka-san, Xie-san, Kozuka-san, Kwon-san, Wu-san, Kim-san and Kim-san, Kurita-san, Nakanishi-san, Harashima-san, Taro-san, Kawamurasan, Ota-san and Sato-san, thanks for the good times.

Tot slot heb ik me ook binnen IMS altijd prima vermaakt. In de eerste plaats door de fijne kantoorgenoten, Paul, Peter en Xin. Verder wil ik met name Jeroen, Joost, Josée, Andreas, Bouwe en Brian bedanken voor de gezellige COMAT etentjes, de Veen, Dekkers, Veldhorst, Steenwelle, Smith, Smithers, Huijben, Broekmaat, van Zalk en Groenen voor de voetbalwedstrijden, Kees voor welkome momenten 
van slap geouwehoer en Matthijn, Paul, Ruud, Peter, Rik, Frank!, Nicholas en natuurlijk Maas voor enige memorabele avonden bier drinken.

Dan is dit toch echt het einde van dit boekwerk. Ik ga op vakantie.

Hans 